\title{
FUNCTION AND GENE EXPRESSION OF CIRCULATING NEUTROPHILS IN DAIRY COWS: IMPACT OF MICRONUTRIENT SUPPLEMENTATION
}

A Dissertation Presented to
The Faculty of the Graduate School
At The University of Missouri
In Partial Fulfillment
Of the Requirements for the Degree
Doctor of Philosophy

By

Xavier S. Revelo

Dr. Matthew R. Waldron, Dissertation Supervisor

July, 2012 
${ }^{\circ}$ Copyright by Xavier S. Revelo 2012

All rights reserved 
The undersigned, appointed by the dean of the Graduate School, have examined the dissertation entitled:

\section{FUNCTION AND GENE EXPRESSION OF CIRCULATING NEUTROPHILS IN DAIRY COWS: IMPACT OF MICRONUTRIENT SUPPLEMENTATION}

\section{Presented by Xavier Revelo}

A candidate for the degree of Doctor of Philosophy in Animal Sciences And hereby certify that, in their opinion, it is worthy of acceptance.

Dissertation Examination Committee:

Advisor

Matthew R. Waldron, Ph.D.

Kevin L. Fritsche, Ph.D.

Matthew C. Lucy, Ph.D.

James W. Perfield, Ph.D. 


\section{ACKNOWLEDGEMENTS}

First of all, I would like to thank my family for their patience, love and inseparable support even after all the years that I have been away from home. Thanks to my mother Sonia for dedicating her life to the guidance, inspiration, and nurturance of our family. I have been blessed with a loving and devoted family who gave me the motivation I needed to pursue my professional goals. I would also like to offer my sincerest gratitude to the friends I made while pursuing my doctorate degree at the University of Missouri. Special thanks to Daniel Mathew, Brad Scharf, Ky Pohler, Eric Coate, and Emma Jinks for sharing their time and friendship. I have great memories of my time in Missouri and will never forget all the trouble we produced during the prime years of our lives.

I would also like to express my appreciation to all the people involved in the research presented in this dissertation. In this regard, I would like to thank former and present members of the Waldron lab who contributed to my research. In particular, I would like to acknowledge Ann Kenny and Nicole Barkley for their hard work during the execution of the animal studies. In a similar way, my most cordial gratitude to Nathan Bivens and Wade Davis for their assistance with the execution and analysis of the RNAsequencing experiments. I would also like to thank all the faculty and staff at the Division of Animal Science for their academic and technical support during my tenure at the University of Missouri.

I would like to thank the members of my graduate program defense committee Kevin Fristche, Matthew Lucy, and James Perfield for their help and input in the realization of this dissertation. Not only they have contributed to my career as teachers 
and mentors, but also cared about me as a person throughout my graduate program at Mizzou. Finally, special thanks to my advisor Matthew R. Waldron for the opportunity to conduct research in his laboratory and for all the guidance received throughout my graduate program. 


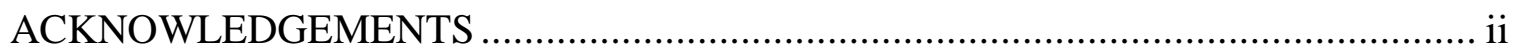

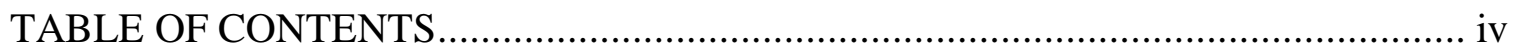

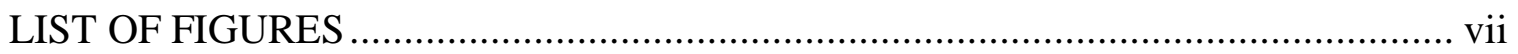

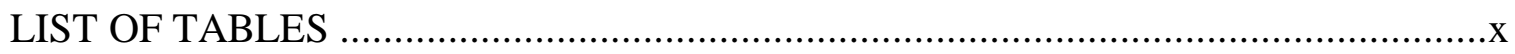

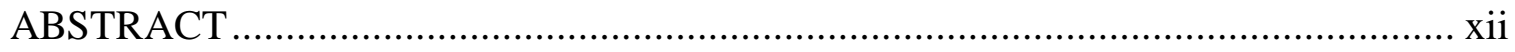

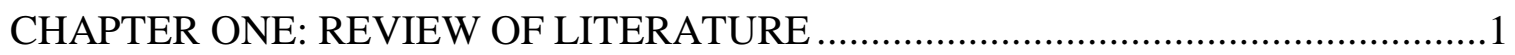

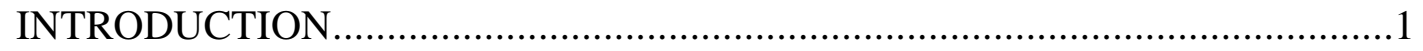

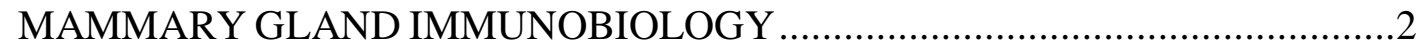

IMMUNE DYSFUNCTION DURING THE PERIPARTURIENT PERIOD ..........16

NUTRITIONAL APPROACHES TO IMPROVE IMMUNITY ............................33

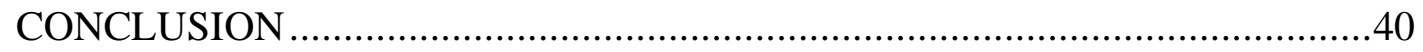

CHAPTER TWO: IN VITRO EFFECTS OF ESCHERICHIA COLI LIPOPOLYSACCHARIDE ON THE FUNCTION AND GENE EXPRESSION OF NEUTROPHILS ISOLATED FROM THE BLOOD OF DAIRY COWS . .43

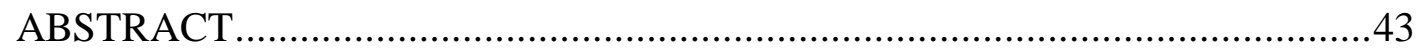

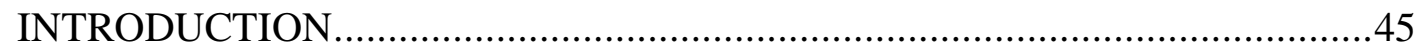

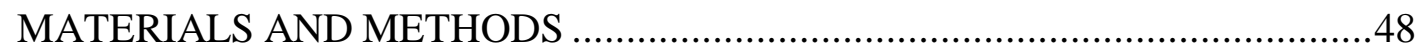

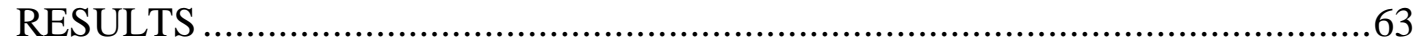

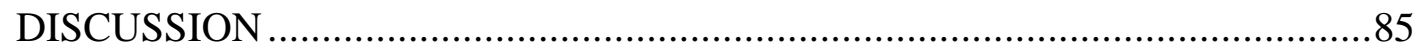

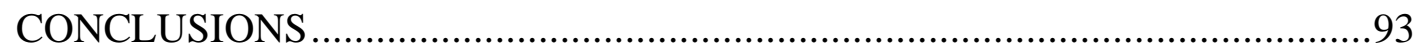

CHAPTER THREE: EFFECT OF A B-VITAMIN AND FUNGAL DIETARY SUPPLEMENT ON THE FUNCTION OF NEUTROPHILS HARVESTED FROM

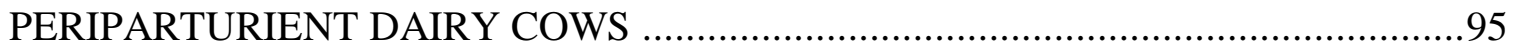

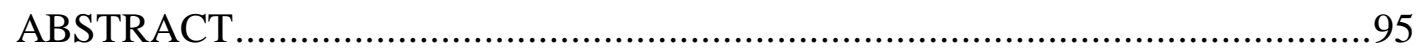

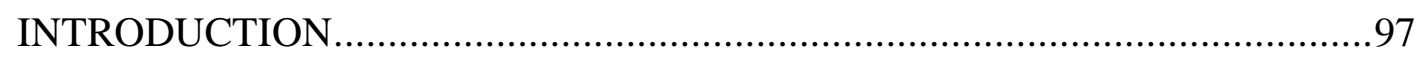

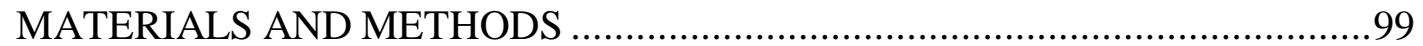

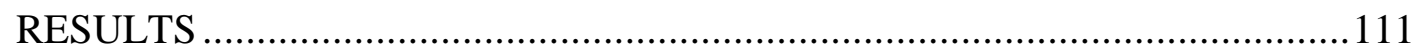




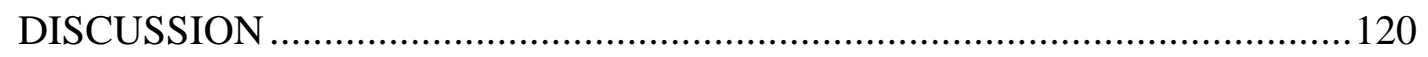

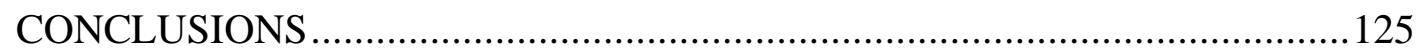

CHAPTER FOUR: EFFECT OF A B-VITAMIN AND FUNGAL DIETARY SUPPLEMENT ON THE GLOBAL GENE EXPRESSION PROFILE OF NEUTROPHILS HARVESTED FROM PERIPARTURIENT DAIRY COWS. 127

ABSTRACT

CHAPTER FIVE: EFFECT OF TRACE MINERAL SUPPLEMENTATION SOURCE ON THE FUNCTION OF NEUTROPHILS COLLECTED FROM PERIPARTURIENT DAIRY COWS.

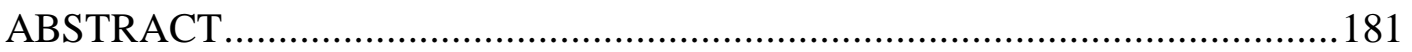

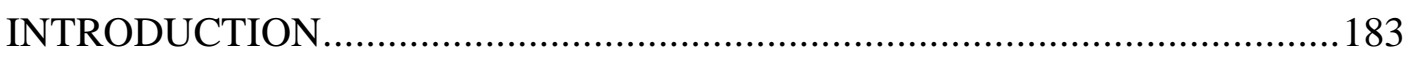

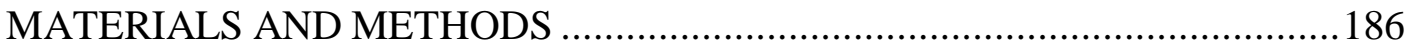

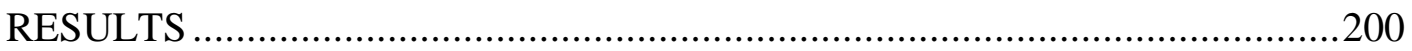

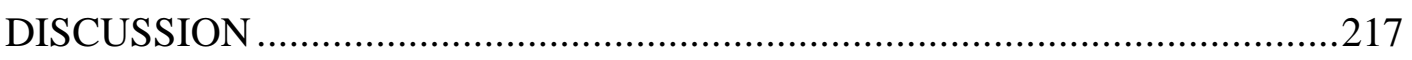

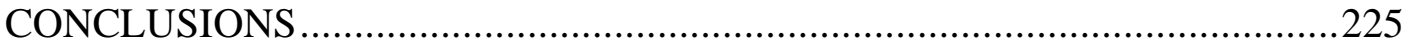

CHAPTER SIX: CHAPTER SIX: EFFECT OF TRACE MINERAL SUPPLEMENTATION SOURCE ON THE GLOBAL GENE EXPRESSION PROFILE OF NEUTROPHILS COLLECTED FROM PERIPARTURIENT DAIRY COWS ......227

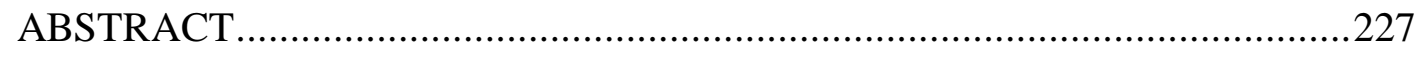

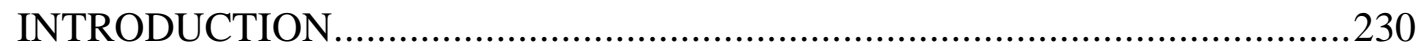

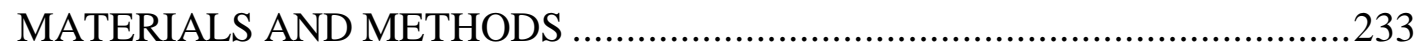

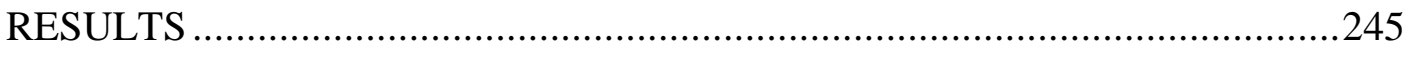

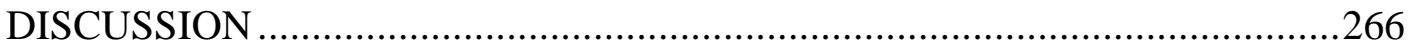

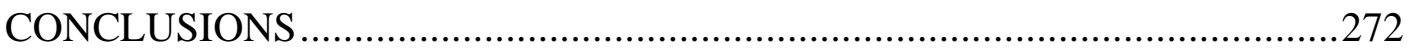


CHAPTER SEVEN: INTREGRATED DISCUSSION FROM THE STUDY OF NUTRITIONAL TACTICS TO IMPROVE INNATE IMMUNITY IN DAIRY COWS

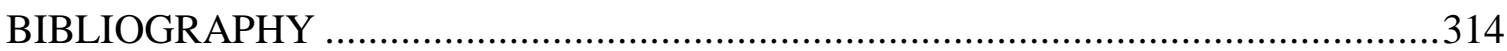

VITA 


\section{LIST OF FIGURES}

Figure 2.1. Effect of Escherichia coli lipopolysaccharide (LPS) on an index of the intracellular reactive oxygen species (ROS) production by bovine neutrophils (PMNL).

Figure 2.2. Effect of Escherichia coli lipopolysaccharide (LPS) on an index of the total reactive oxygen species (ROS) production by bovine neutrophils (PMNL). 69

Figure 2.3. Effect of Escherichia coli lipopolysaccharide (LPS) on neutrophil extracellular traps expression (NETs). .72

Figure 2.4. Effect of Escherichia coli lipopolysaccharide (LPS) on the chemotaxis of bovine neutrophils (PMNL) towards the chemoattractant interleukin-8 (IL8).

Figure 2.5. Effect of Escherichia coli lipopolysaccharide (LPS) on the bactericidal ability of neutrophils (PMNL) against Staphylococcus aureus. 75

Figure 2.6. Quantitative real-time PCR analysis of the expression of the inflammatory cytokines interleukin-8 (IL-8) and tumor necrosis factor (TNF) in neutrophils (PMNL).

Figure 2.7. Quantitative real-time PCR analysis of bactericidal/permeability increasing protein (BPI) in neutrophils (PMNL). 79

Figure 2.8. Quantitative real-time PCR analysis of the expression of the proteins involved in the production of reactive oxygen species myeloperoxidase (MPO), superoxide dismutase 2 (SOD2), NADPH oxidase 4 (NOX4), and cytochrome b-245, alpha polypeptide (CYBA) in neutrophils (PMNL).....81

Figure 2.9. Quantitative real-time PCR analysis of the mRNA expression of the histones $H 2 A / 1$ and $H 2 B$, structural components of neutrophil (PMNL) extracellular traps.

Figure 3.1. Effect of a dietary supplement containing B-complex vitamins and yeast on the reactive oxygen species (ROS) production by bovine neutrophils (PMNL). .113

Figure 3.2. Effect of a dietary supplement containing B-complex vitamins and yeast on Neutrophil Extracellular Trap release (NET) by bovine neutrophils (PMNL).

Figure 3.3. Effect of a dietary supplement containing B-complex vitamins and yeast on neutrophil (PMNL) chemotaxis towards the chemoattractant interleukin-8 (IL-8). 117 
Figure 3.4. Effect of a dietary supplement containing B-complex vitamins and yeast on the neutrophil (PMNL) killing ability against Staphylococcus aureus...... 119

Figure 4.1. Effect of supplementing cows with OmniGen-AF ${ }^{\circledR}$ on the lysosome pathway in neutrophils (PMNL) after activation with Escherichia coli lipopolysaccharide (LPS).

Figure 4.2. Effect of supplementing cows with OmniGen-AF ${ }^{\circledR}$ on the oxidative phosphorylation pathway in neutrophils (PMNL) regardless of incubation with Escherichia coli lipopolysaccharide (LPS). 148

Figure 4.3. Effect of Escherichia coli lipopolysaccharide (LPS) on the toll-like receptor (TLR) signaling pathway in neutrophils (PMNL) regardless of dietary supplementation.

Figure 4.4. Effect of Escherichia coli lipopolysaccharide (LPS) on the hematopoietic cell lineage signaling pathway in neutrophils (PMNL) regardless of dietary supplementation. 152

Figure 5.1. Effect of trace mineral supplementation source on an index of the total reactive oxygen species (ROS) production by bovine neutrophils (PMNL). .202

Figure 5.2. Effect of trace mineral supplementation source on Neutrophil Extracellular Trap release (NET) by bovine neutrophils (PMNL). 205

Figure 5.3. Effect of trace mineral supplementation source on neutrophil (PMNL) chemotaxis towards the chemoattractant interleukin-8 (IL-8). .208

Figure 5.4. Effect of trace mineral supplementation source on neutrophil (PMNL) phagocytosis of Escherichia coli particles.

Figure 5.5. Effect of trace mineral supplementation source on the neutrophil (PMNL) killing ability against Escherichia coli. 215

Figure 6.1. Regulation of the RIG-I-like receptor signaling pathway in neutrophils (PMNL) from cows supplemented with trace minerals from organic sources relative to those fed inorganic trace minerals. 248

Figure 6.2. Regulation of the cytosolic DNA-sensing pathway in neutrophils (PMNL) from cows supplemented with trace minerals from organic sources relative to those fed inorganic trace minerals. 250

Figure 6.3. Regulation of the TOLL-like receptor signaling pathway in neutrophils (PMNL) from cows supplemented with trace minerals from organic sources relative to those fed inorganic trace minerals. 252 
Figure 6.4. Non-activated neutrophils (PMNL) from postpartum cows have a defective chemokine signaling pathway compared with PMNL from prepartum

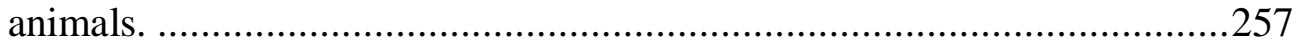

Figure 6.5. Non-activated neutrophils (PMNL) from postpartum cows have a defective leukocyte transendothelial migration pathway compared with PMNL from prepartum animals.

Figure 6.6. Non-activated neutrophils (PMNL) from postpartum cows have a defective Fc gamma R-mediated phagocytosis pathway compared with PMNL from

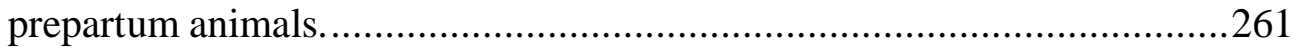




\section{LIST OF TABLES}

Table 2.1. Gene description, symbol and relevant biological functions or processes....57

Table 2.2. Accession number, primer sequences and percentage of similarity between amplified RT-PCR products and publicly available sequences by the National Center for Biotechnology Information (NCBI) ..........................60

Table 3.1. Composition of the dietary supplement Omnigen- $\mathrm{AF}^{\circledR}$............................. 102

Table 3.2. Chemical composition of the prepartum and postpartum diets (DM basis) 103

Table 4.1. Composition of the dietary supplement Omnigen-AF ${ }^{\circledR}$.......................... 133

Table 4.2. Chemical composition of the prepartum and postpartum diets (DM basis) 134

Table 4.3. Conversion and quality control summary 139

Table 4.4. Summary of sequencing read alignment to the reference bovine genome..140

Table 4.5. Enriched annotation terms for genes differentially regulated due to dietary supplementation with $56 \mathrm{~g} /$ day of either OmniGen-AF ${ }^{\circledR}(\mathrm{n}=5)$ or sham control $(\mathrm{n}=5)$ in neutrophils $(\mathrm{PMNL})$ treated with 0 or $50 \mu \mathrm{g} / \mathrm{mL}$ of Escherichia coli lipopolysaccharide (LPS) 155

Table 4.6. Supplemental. Differential expression of genes in neutrophils (PMNL) from cows fed either $56 \mathrm{~g}$ /day of either the supplement OmniGen-AF${ }^{\circledR}(\mathrm{n}=5)$ or sham control (soybean hulls; $\mathrm{n}=5$ ) upon a 120-min incubation with 0 or 50 $\mu \mathrm{g} / \mathrm{mL}$ of Escherichia coli lipopolysaccharide (LPS) 164

Table 5.1. Chemical composition of the basal prepartum and postpartum diets (DM basis) 188

Table 6.1. Chemical composition of the basal prepartum and postpartum diets (DM basis)

Table 6.2. Conversion and quality control summary

Table 6.3. Summary of sequencing read alignment to the reference bovine genome..242

Table 6.4. Enriched pathways for genes with differential expression due to physiological state (postpartum vs. prepartum) after incubation with 0 or 50 $\mu \mathrm{g} / \mathrm{mL}$ of $E$. coli lipopolysaccharide (LPS)

Table 6.5. Enriched annotation terms for genes differentially regulated due to trace mineral supplementation from organic sources (relative to inorganic sources) 
in neutrophils (PMNL) treated with 0 or $50 \mu \mathrm{g} / \mathrm{mL}$ of Escherichia coli lipopolysaccharide (LPS) .............................................................2 264

Table 6.6. Supplemental. Differential expression of genes in neutrophils (PMNL) collected from cows fed no added dietary trace minerals $(n=7)$ or supplemented with trace minerals from either inorganic $(n=7)$ or organic $(n$ $=7$ ) sources after incubation with 0 or $50 \mu \mathrm{g} / \mathrm{mL}$ of $E$. coli lipopolysaccharide for $120 \mathrm{~min}$ 274

Table 6.7. Supplemental. Top 100 genes with differential expression in neutrophils (PMNL) collected from cows either on day $58.1 \pm 1.0$ prepartum or $6.4 \pm 0.1$ postpartum after incubation with 0 or $50 \mu \mathrm{g} / \mathrm{mL}$ of $E$. coli

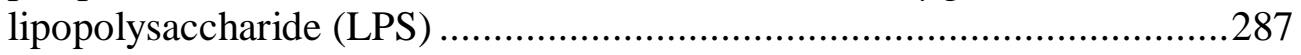

Table 7.1. Summary of studies of neutrophil (PMNL) reactive oxygen species (ROS) production, release of extracellular traps (NETs), directed chemotaxis, killing of bacteria, and phagocytosis. 309 


\title{
FUNCTION AND GENE EXPRESSION OF CIRCULATING NEUTROPHILS IN DAIRY COWS: IMPACT OF MICRONUTRIENT SUPPLEMENTATION
}

\author{
Xavier S. Revelo \\ Dr. Matthew R. Waldron, Dissertation Supervisor
}

\begin{abstract}
Three experiments were conducted to investigate the mechanisms of neutrophil dysfunction during the periparturient period and test potential nutritional strategies to prevent the immune-alteration typical of this time. In the first experiment, the in vitro effects of Escherichia coli lipopolysaccharide (LPS) on the function of bovine neutrophils (PMNL) and expression of selected genes were examined. Lipopolysaccharide directly stimulated PMNL from midlactation cows $(\mathrm{n}=7)$ to produce reactive oxygen species (ROS), and release of neutrophil extracellular traps (NETs), but did not alter their chemotaxis and killing of $S$. aureus. Furthermore, LPS stimulated the gene expression of tumor necrosis factor- $\alpha$ (TNF), bactericidal/permeability increasing protein (BPI) and cytochrome $b(\mathrm{CYBA})$ in PMNL from midlactation $(\mathrm{n}=10)$, but not from early lactation $(n=10)$ cows. Regardless of stage of lactation, LPS also increased the mRNA contents of interleukin-8 (IL-8) and superoxide dismutase 2 (SOD2). In the second experiment, the effects of a B-vitamin dietary and yeast supplement on the function and global gene expression of PMNL from periparturient dairy cows were tested. Cows received $56 \mathrm{~g} /$ day of either OmniGen- $\mathrm{AF}^{\circledR}(\mathrm{n}=8)$ or sham control $(\mathrm{n}=12)$ from day 46 before calving until day 31 after parturition. PMNL function was assessed on days 49, 28, and 19 prepartum, and 1, 7, 14 and 30 postpartum. In addition, changes in global gene expression due to Omnigen-AF ${ }^{\circledR}$ supplementation were examined on day 7
\end{abstract}


postpartum after PMNL incubation with 0 or $50 \mu \mathrm{g} / \mathrm{mL}$ of $E$. coli LPS. Although no differences in PMNL function were detected between supplemented and nonsupplemented cows, Omnigen- $\mathrm{AF}^{\circledast}$ down-regulated the expression of genes that enriched the lysosome pathway (upon activation with LPS) and decreased the expression of transcripts in the oxidative phosphorylation pathway (regardless of LPS activation). Independent of dietary supplementation, LPS activation stimulated the TOLL-like receptor signaling and hematopoietic cell lineage pathways.

The objective of the third study was to investigate the effects of trace mineral supplementation from inorganic or organic sources on PMNL function and global gene expression in dairy cows during the periparturient period. Thirty nine pregnant Holstein cows were assigned to a basal diet with no added dietary $\mathrm{Mn}, \mathrm{Co}, \mathrm{Cu}$ or $\mathrm{Zn}$ (basal, $\mathrm{n}=$ 13) or supplemented with $200 \mathrm{mg} \mathrm{Mn}, 25 \mathrm{mg} \mathrm{Co}, 125 \mathrm{mg} \mathrm{Cu}$, and $360 \mathrm{mg} \mathrm{Zn}$ from inorganic $(\mathrm{n}=11)$ or organic $(\mathrm{n}=11)$ sources. Neutrophils were collected from the blood of cows on days 58 and 7 prepartum, and 6 postpartum to determine their function and global gene expression (days 58 prepartum and 6 postpartum only, $\mathrm{n}=7$ ) after incubation with 0 or $50 \mu \mathrm{g} / \mathrm{mL}$ of $E$. coli LPS. Cows supplemented with trace minerals from inorganic sources had a $39 \%$ increase in the amount of E. coli particles phagocytized by PMNL on day 6 postpartum, compared with cows receiving no supplemental trace minerals. There was no difference in any other aspect of PMNL function measured between animals supplemented with inorganic or organic forms of trace minerals and those receiving no supplementation. Additionally, changes in global gene expression of PMNL due to trace mineral supplementation source were examined on days -58 and 6 , relative to parturition. Trace mineral supplementation from either inorganic or organic 
sources resulted in minimal changes in gene expression in non-activated and LPSactivated PMNL, relative to cows fed the basal diet. However, organic trace mineral supplementation (vs. inorganic) up-regulated the expression of genes that enriched the RIG-I-like receptor signaling, cytosolic DNA-sensing, and TOLL-like receptor (TLR) signaling pathways. Together the results from the third experiment suggest that trace mineral supplementation, regardless of source, had minimal effects on PMNL function and gene expression. However, organic trace minerals, relative to those from inorganic sources, stimulated genes involved in antigen recognition and immune response. In conclusion, we identified several cellular and molecular mechanisms of bovine PMNL dysfunction during the periparturient period. In addition, several pathways by which supplementation with micronutrients may impact PMNL performance were discovered. 


\section{CHAPTER ONE}

\section{REVIEW OF LITERATURE}

\section{INTRODUCTION}

Infectious diseases such as mastitis reduce the profitability of dairy farming as due to decreased milk production and increased expenditure in veterinary services, diagnostics, drugs, prevention, and extra labor (Hogeveen et al., 2011). In the United States, the economic losses due to mastitis surpass two billion dollars each year (NMC, 1996). At the individual cow level, the average cost of clinical mastitis is estimated at $\$ 155$ per case (Cha et al., 2011). Although the understanding of the biology of this disease has increased in the last few decades, the incidence and severity of mastitis have not declined (LeBlanc et al., 2006). For instance, the National Animal Health Monitoring Service reported that the prevalence of clinical mastitis in the U. S. increased from 13.4 to $16.5 \%$ between 1996 and 2007 (USDA, 2008). The resolution of clinical cases of mastitis is determined largely by cow factors and depends in particular on the ability of polymorphonuclear cells (PMNL) or neutrophils to recognize the insult, migrate into the mammary gland, phagocytose and ultimately eliminate the pathogen (Vangroenweghe et al., 2005). Remarkably, dairy cows are particularly susceptible to mastitis for several weeks around the time of parturition in part due to an incompetent immune system and increased oxidative stress (Mallard et al., 1998; Sordillo and Aitken, 2009). The altered functional competence of the immune system during the periparturient period cannot be explained by a single physiological event. Instead, several metabolic and endocrine changes that occur during the transition period seem to contribute to this immune 
dysfunction (Overton and Waldron, 2004). Improving our understanding of periparturient immunobiology and the roots of immunosuppression will facilitate the development of interventions to reduce the incidence and severity of clinical mastitis in dairy cows. In addition, nutritional strategies such as dietary supplementation with several micronutrients may boost the host immune response and enhance resistance against intramammary infections.

\section{MAMMARY GLAND IMMUNOBIOLOGY}

The mammary gland is protected by immune defense mechanisms that can be classified into two categories: innate and adaptive immunity. The innate immune system is also known as the non-specific immune system. However, the innate immune response is not entirely non-specific as frequently described. Instead, cells of the innate immune system are able to discriminate between self and a variety of pathogens using a limited number of receptors (Akira et al., 2006). Innate immunity serves as the first line of defense during the early stages of intramammary infection. If the innate immune system fails to completely eliminate the pathogen, the adaptive immune system acts as a second line of defense that is highly specific and able to form immunological memory (Sordillo and Streicher, 2002). Despite these differences, innate and adaptive immunity systems interact closely to enhance the protection against the insult. For instance, macrophages function as antigen presenting cells (APCs) in addition to their role as innate phagocytes. In association with major histocompatibility complex (MHC) class II antigens, APCs process and present antigens from ingested bacteria on their membrane. These MHC II molecules are required by cells of the adaptive immune system for recognition of foreign 
antigens (Sordillo et al., 1997). However, it is now evident that the crosstalk between innate and adaptive arms of the immune system is not limited to the interaction between APCs and lymphocytes. Innate instruction of adaptive immunity controls the activation, type, and duration of the adaptive immune response. Interestingly, this instruction of adaptive immunity by the innate immune immunity occurs throughout the immune response and involves most cellular components of the immune system (Schenten and Medzhitov, 2011). In the dairy cow, the efficiency of the innate immune system often determines the outcome of the intramammary infection. Unlike the adaptive immune system, which requires several days or weeks to respond to the insult, the innate immune system must attempt to eliminate the pathogens from the gland immediately within hours after infection (Bannerman, 2009).

\section{Innate immune defense mechanisms}

The components of the innate immune system include barriers that protect the host (e.g. skin, mucosal membranes, acidity of the stomach, perspiration, etc.), soluble factors (complement system, the enzyme lysozyme, and interferon proteins) and cellular components such as neutrophils, macrophages, and natural killer cells (Akira et al., 2006). In the mammary gland of dairy cows, the teat canal is considered the first physical barrier against the invading pathogen. Sphincter muscles in the teat canal maintain closure of the orifice and prevent bacterial penetration. In addition, the teat canal is lined with keratin which can associate with proteins and fatty acids that can act as bacteriostatics (Sordillo et al., 1997). Thus, the teat canal is a critical barrier against intramammary infections and damage to this structure is a predisposing factor for clinical mastitis (Myllys et al., 1994). 
If the invading pathogen is able to evade the first functional barriers and colonize the udder cistern, the innate immune system relies on a limited set of pattern recognition receptors (PRRs) to detect and mount a defensive response. The TOLL-like receptors (TLR), a subtype of PRRs, are used by resident macrophages and other APCs to recognize and bind highly conserved molecular motifs known as pathogen-associated molecular patterns or PAMPs (Werling et al., 2006). Cells of the innate immune system can use their TLRs to discern between distinct PAMPs to discriminate between pathogens. For example, growth and death of Gram-negative bacteria results in the shedding of the lipopolysaccharide components of the bacterial cell wall (LPS) which can be recognized by TLR-4 in conjunction with the accessory molecules LBP, CD14 and MD-2 (De Schepper et al., 2008; Worku and Morris, 2009). In contrast, lipoteichoic acid (LTA) is the main component of the cell wall of Gram-positive bacteria (Aderem and Ulevitch, 2000) and its recognition is mediated by TLR-2 which has been detected on the surface of bovine monocytes, monocyte-derived macrophages and dendritic cells (Kwong et al., 2011).

In the infected mammary gland, recognition of PAMPs by resident macrophages and epithelial cells triggers the inflammatory response via secretion of pro-inflammatory cytokines into the surrounding environment (Bannerman, 2009). Although their relevance in the dairy cow is unclear, cytosolic multi-protein complexes termed inflammasomes are receiving increasing attention as key activating platforms of the host response against the invading pathogens (Lamkanfi and Dixit, 2011). In humans and animal models, assembly of these inflammasomes induces the maturation and secretion of pro-inflammatory cytokines such as interleukin (IL)-1 $\beta$ and IL-18 via caspase-1 activation. In addition, 
inflammasomes prevent bacterial multiplication and remove infected immune cells in a novel form of cell death called pyroptosis (Vande Walle and Lamkanfi, 2011). In the infected mammary gland, activated phagocytes and epithelial cells produce and release several soluble cytokines and non-cytokine inflammatory mediators including the complement-cleavage product C5a, tumor necrosis factor (TNF)- $\alpha$, IL-1, IL-2, IL-6, IL-8, and interferons that trigger the initiation of the inflammatory reaction and promote the migration of leukocytes to the site of infection (Riollet et al., 2000; Bannerman, 2009).

The inflammatory mediators released during the early stages of infection activate the neutrophils in circulation which are rapidly recruited to the mammary gland. As an example, intramammary infusion with LPS results in a dramatic influx of somatic cells mostly consisting of neutrophils into the mammary gland as early as 4 hours after the challenge (Bannerman et al., 2003). Therefore, neutrophils are the predominant cellular population in the infected mammary gland whereas macrophages and monocytes comprise the majority of leukocytes before the insult (Saad and Ostensson, 1990). Importantly, the ability of these neutrophils to recognize and destroy the invading pathogen plays an early role in determining the severity and duration of the infection (Sordillo and Streicher, 2002).

\section{Role of neutrophils in the resolution of intramammary infections}

Neutrophils are a primary component of the innate immune response. Efficient migration of neutrophils into the mammary gland and subsequent antimicrobial activity are crucial for the resolution of an intramammary infection (Vangroenweghe et al., 2005). Once circulating neutrophils sense specific signals such as chemokines or bacterial products, they migrate up the concentration gradient of these chemoattractants. 
Neutrophils then adhere to, and pass through the vascular endothelium in a sequential process known as extravasation or diapedesis (Paape et al., 2002). The initial step in neutrophil extravasation is the capture or tethering mediated by the adhesion molecules L-, E-, and P-selectin which slow the neutrophils at the vessel wall. The adhesion molecule L-selectin is expressed by most leukocytes whereas E- and P-selectin are expressed by inflamed endothelium and platelets (Schmidt et al., 2011). After this initial capture, neutrophils roll on the endothelium until enhanced affinity promotes firm adhesion or leukocyte arrest. This high affinity binding is mediated by the interaction between the adhesion molecules $\beta 2$-integrin CD11a/CD18 (LFA-1) and the intercellular adhesion molecule (ICAM)-1 (Diez-Fraile et al., 2002). The neutrophil then crawls along endothelial cells and migrates across the endothelium by passing between or through endothelial cells during paracellular or transcellular migration, respectively. In paracelullar migration, the neutrophil extends its lamelopodia between endothelial cells to disrupt and translocate through the tight junctions. During transcellular migration the neutrophil forms actin-rich podosomes that indent the endothelium which in turn forms projections around the neutrophil known as the transmigratory cup to facilitate crossing of the leukocyte (Schmidt et al., 2011). Although transcellular migration requires extensive re-organization of the cytoskeleton of both neutrophils and endothelial cells, minimal vascular leak is caused as the endothelium forms a dome that promotes membrane closure (Woodfin et al., 2010).

Upon crossing the blood-milk barrier, the first event that occurs in the process of phagocytosis is recognition of pathogens. Invading bacteria are marked for ingestion by the binding of opsonins including antibodies, mainly immunoglobulin $\mathrm{M}(\operatorname{IgM})$ and $\operatorname{IgG}_{2}$, 
and complement fragments. Subsequently, neutrophils can use their Fc receptors (FcR) and complement receptors (CR) to bind the opsonized pathogens and initiate phagocytosis (Paape et al., 2002). Although opsonization is not essential for phagocytosis, it promotes uptake of pathogens and enhances neutrophil functions. For instance, in vitro phagocytosis of Staphylococcus aureus by neutrophils increased by 58 and $32 \%$ when the bacteria were opsonized with $\operatorname{IgM}$ and $\operatorname{IgG} 2$, respectively, compared with phagocytosis of non-opsonized S. aureus (Guidry et al., 1993). In addition, costimulation with $\mathrm{CR} 3$ and $\mathrm{Fc} \gamma \mathrm{R}$ ( $\mathrm{Fc}$ receptor for $\mathrm{IgG}$ ) enhances superoxide production, intracellular calcium, and enhanced phosphorylation by mitogen-activated neutrophils (Nagahata et al., 2007). Interestingly, bovine neutrophils are also capable to phagocytize microbes in the absence of opsonins through specific binding between mannose-specific lectins on the surface of the pathogen and carbohydrates on the membrane of the neutrophil (Paape et al., 1996). Regardless of the receptors involved, binding of neutrophils to the pathogen leads to intracellular signaling that initiates actin polymerization, myosin $\mathrm{X}$ activation, and membrane remodeling. These early events promote the localized delivery of endomembranes and development of the phagosomal cup which surrounds the antigen to form the phagosome (Lee et al., 2003). Initially, this nascent phagosome has no antimicrobial capacity, however, it undergoes rapid maturation to acquire lysosome-like properties and receive the contents of preformed granules (Nordenfelt and Tapper, 2011). In addition to phagocytosis, pathogen recognition triggers other antimicrobial measures to eliminate the insult including the intracellular and extracellular release of toxic oxygen radicals and granular enzymes (Paape et al., 2002). As a final act, neutrophils can extrude granular proteins and fibrils of 
decondensed chromatin to form extracellular structures known as extracellular traps or NETs (Brinkmann et al., 2004).

\section{Production of reactive oxygen and nitrogen species by neutrophils}

In the infected tissue, one of the weapons that neutrophils use to eliminate the invading pathogen is the production and discharge of reactive oxygen species (ROS), reactive nitrogen species (RNS), and antimicrobial proteins (Paape et al., 2003). Within the cytoplasm of the bovine neutrophil, antimicrobial compounds are stored in primary, secondary, and tertiary granules. Primary or azurophilic granules contain the enzyme myeloperoxidase which is involved in the production of reactive oxygen species (ROS). In contrast, secondary granules are rich in lactoferrin whereas tertiary granules contain the majority of proteins with antimicrobial activity (Paape et al., 2003). Upon activation, neutrophils can discharge the contents of their storage granules both into phagosomes and to the extracellular space. However, neutrophils are not limited to the release of granular contents into the phagosome or outside the cell. Instead, activated neutrophils have a dramatic increase in the turnover of the hexose monophosphate shunt that leads to nonmitochondrial respiratory burst activity and production of ROS mediated by the enzyme complex NADPH oxidase (Shepherd, 1986).

The core component of the enzyme complex NADPH oxidase is the flavocytochrome b558, located in the membrane of specific granules of the resting neutrophil. Upon activation, flavocytochrome b558 is incorporated into the wall of the phagocytic vacuole where it carries electrons from NADPH in the cytoplasm to $\mathrm{O}_{2}$ inside the vacuole. During electron transport, flavocytochrome interacts with other components of the complex including p67phox, p47phox, p40phox and p21rac, which also translocate 
to the membrane of the vacuole (Segal, 2005). Thus, the NADPH oxidase complex catalyzes the consumption of $\mathrm{O}_{2}$ via electron transfer from $\mathrm{NADPH}$ to $\mathrm{O}_{2}$ to generate superoxide anion $\left(\mathrm{O}_{2}{ }^{-}\right)$. In turn, superoxide dismutase converts $\mathrm{O}_{2}{ }^{-}$to $\mathrm{H}_{2} \mathrm{O}_{2}$ which is the main precursor for numerous ROS (Hodgson et al., 2006). The enzyme myeloperoxidase then forms a complex with $\mathrm{H}_{2} \mathrm{O}_{2}$ which attacks the pathogen directly or reacts with nitrogen intermediates to form RNS (Shepherd, 1986). The lysosomal vacuoles containing ROS and RNS will fuse with the neutrophil phagosomes containing the phagocytized pathogen bacteria. This fusion creates a mature phagosome or phagolysosome in which the pathogen can be killed via exposure to antimicrobial molecules.

The ability of the neutrophils to produce ROS and RNS is critical to ensure the elimination of the invading pathogen. However, neutrophil migration into the mammary gland during infection has the potential to cause chemical and/or mechanical damage to the milk secretory cells (Akers and Nickerson, 2011). Neutrophils may promote tissue injury and affect mammary function via oxidative stress due to the discharge of ROS and other antimicrobial compounds to the extracellular matrix (Zhao and Lacasse, 2008). For example, Capuco et al. (1986) obtained mammary tissue explants at slaughter from noninfected quarters of five lactating cows and incubated them with intact, lysed or zymozan-activated neutrophils isolated from mammary secretions of nulliparous heifers. These authors reported that explants treated with lysed and activated neutrophils presented less alveolar luminal area, swollen milk secretory cells with nuclear pyknosis, epithelial vacuolation, and filling of luminal areas with cell debris, compared with control tissue. Therefore, a fine balance must exist between a vigorous immune response required 
to eliminate the invading pathogen and the activation of anti-inflammatory mechanisms, indispensable to reinstate mammary gland immune homeostasis (Aitken et al., 2011). Neutrophil extracellular traps (NETs)

Neutrophil extracellular trap release was first reported by Brinkmann et al. (2004) as a novel antimicrobial mechanism and opened a new phase in the study of neutrophil function and morphology. Several pathogens and pro-inflammatory mediators including LPS, IL-8, and TNF- $\alpha$ can stimulate the formation and release of NETs. Activation of neutrophils leads to swelling of the nuclei and dissolution of its chromatin to form strands of decondensed DNA (Brinkmann et al., 2004). Subsequently, rupture of nuclear and granular membranes allows mixing of these structures with granule substances including proteolytic enzymes, antimicrobial proteins, and ROS. NETs are then released to the extracellular matrix where they contribute to the killing ability of PMNL by delivering a high concentration of antimicrobial substances and by forming a barrier that prevents the microorganisms from spreading (Brinkmann et al., 2004; Brinkmann and Zychlinsky, 2007).

Nucleic and cytoplasmic proteins found in NETs which are thought to provide their killing include histones, elastase, myeloperoxidase, pentraxin, lactoferrin, and bactericidal/permeability-increasing protein (Lögters et al., 2009). Although it was initially proposed that NETs are released by intact viable cells, recent evidence indicates that PMNL extrude NETs in a processes of cell death termed NETosis which is different from apoptosis and necrosis (Brinkmann and Zychlinsky, 2007). Contrary to apoptotic cells, neutrophils do not express engulfment or "eat-me" markers (e.g., phosphatidylserine) during NETosis preventing premature clearance by phagocytes. Also, 
in contrast to programmed necrosis, the integrity of the plasma membrane is maintained during NETosis although nuclear and granular membranes disintegrate to allow mixing of antimicrobial compounds with nuclear chromatin (Remijsen et al., 2011). Importantly, NETs may have significant roles in disease as they are abundant at sites of infection during appendicitis in humans, and pneumonia and fasciitis in animal models (Wartha et al., 2007). In dairy cows, neutrophils are also capable of releasing NETs upon stimulation with mitogens or mastitis-causing bacteria (Lippolis et al., 2006b). Indeed, NETs may have an important role in the defense against intramammary infections. Incubation of bovine neutrophils in milk does not reduce their ability to release NETs, in contrast with other features of neutrophil function, suggesting that when other PMNL killing mechanisms are impaired, neutrophils might rely on killing of bacteria by NETs (Lippolis et al., 2006b). Despite increasing attention on the biology of NETs and their potential role in disease, a recent study by Menegazzi et al. (2011) has raised doubts about the antimicrobial ability of NETs. In contrast to previous studies in which the disappearance of microbes from culture was assumed to be a result of killing by NETs, these investigators reported that bacteria released from their capture by NETs are alive and proposed that the function of these structures may be limited to the ensnarement and containment of target microbes (Menegazzi et al., 2011). Future research is needed to corroborate or disprove these findings.

\section{Adaptive immune defense mechanisms}

Although the innate and adaptive immune systems both function to protect the mammary gland against invading pathogens, they differ in several aspects. First, the adaptive immune system requires some time to react against the insult and it is only 
triggered if the innate immunity fails to control the infection. Second, the adaptive immune system is capable of recognizing antigenic determinants of a pathogen with a high degree of specificity. Finally, the adaptive immune system retains immunological memory to provide a faster, stronger and longer lasting response upon subsequent exposure to the same antigen (Sordillo and Streicher, 2002). These immunologic attributes of specificity, diversity, and memory are defined by antigen-binding cellsurface receptors expressed on the cell membrane of lymphocytes which are the major cell type of the adaptive immune system. There are two distinct subsets of lymphocytes, the B-lymphocytes (B-cells) and T-lymphocytes (T-cells). The main functions of Blymphocytes include antigen presentation and production of antibodies or immunoglubulins. Antibodies are the soluble form of the B-cell antigen receptor and serve as effectors of humoral immunity when secreted in circulation. The functional properties and biological advantages of immunoglobulins depend on their isotype. For example, IgM is involved in the activation of the complement cascade, IgG promotes the phagocytosis and killing of microbes by phagocytes and transfers passive immunity to the newborn, $\operatorname{IgG}$ and IgA form immune complexes that facilitate the clearance of antigen, and IgE triggers the degranulation of basophils and eosinophils (Nonnecke and Harp, 1989).

Initiation of an adaptive immune response occurs with the interaction between APCs (e.g., B cells, macrophages, and dendritic cells) and T-lymphocytes which recognize foreign antigens via their T-cell receptor (TCR). T-cells can be grouped into two main subsets, cytotoxic T-cells $\left(\mathrm{CTL}, \mathrm{CD} 8^{+}\right)$and helper $\mathrm{T}$-cells $\left(\mathrm{T}_{\mathrm{H}}, \mathrm{CD} 4^{+}\right)$. The role of activated CTL is to exhibit killing or cytotoxic activity against host cells that display 
antigen including infected cells and tumor cells. In contrast, helper T-cells do not have a direct role in the elimination of antigen. Instead, activated $\mathrm{T}_{\mathrm{H}}$ cells become effector cells that secrete a wide variety of cytokines (Lippolis, 2008). The specificity of the TCR is not for the antigen alone, but for the antigen together with the major histocompatibility complex (MHC) class I and II found on the surface of the APC. Major histocompatibility complex class I molecules are expressed by all nucleated cells and present peptides at the cell surface to $\mathrm{CD} 8^{+} \mathrm{T}$ cells. Alternatively, MHC class II molecules are primarily expressed by professional APCs such as dendritic cells, macrophages and B lymphocytes and present epitopes to $\mathrm{CD} 4^{+} \mathrm{T}$ cells (Neefjes et al., 2011). The TCR is a heterodimer composed of either $\alpha$ and $\beta$ or $\gamma$ and $\delta$ chains which is bound to the cell membrane and does not appear in a soluble form. Although in humans the percentage of $\gamma \delta \mathrm{T}$-cells in circulation is small (1 to 10\%), cattle have a much larger proportion of $\gamma \delta$ T-cells in circulation (Burton and Kehrli, 1996). In humans and mice, $\gamma \delta$ T-lymphocytes are neither MCH class I nor II restricted and often do not express CD4 or CD8 (Prinz, 2011). In dairy cows, a high proportion of $\gamma \delta$ T-lymphocytes in the milk of non-mastitic cows express $\mathrm{CD} 8^{+}$regardless of stage of lactaction. In contrast the proportion of $\gamma \delta \mathrm{T}$-cells $\mathrm{CD}^{+}$is low in early lactation but increases as lactation advances (Taylor et al., 1994). Although the role of $\gamma \delta$ T-cells in bovine mammary gland immunity is merely speculative, these $\gamma \delta \mathrm{T}$-cells may provide an important line of defense against intramammary infections due to their cytotoxic abilities (Sordillo and Streicher, 2002).

\section{Mammary gland infecting pathogens}

The primary cause of inflammation of the mammary gland is the invasion and growth of bacteria within the udder (Burvenich et al., 1999). Pathogens that colonize the 
mammary gland and cause mastitic infections can be classified as contagious or environmental pathogens. Contagious pathogens such as Staphylococcus aureus, Staphylococcus chromogenes, and Mycoplasma sp. are mostly transmitted from animal to animal. The main reservoir of $S$. aureus and $S$. chromogenes is the infected gland of heifers and lactating cows (Roberson et al., 1994; Piessens et al., 2011) whereas bedding sand is a significant reservoir of Mycoplasma sp. (Justice-Allen et al., 2010). Transmission of $S$. aureus and $S$. chromogenes can occur either from teat to teat in the same animal or from cow to cow during milking. The vectors involved in this transmission include the hands of milkers, cloths used to clean the udder, and milking cups (Harmon, 1994). Intramammary infections caused by contagious pathogens are usually chronic and subclinical due to the ability of these pathogens to evade host defense mechanisms and penetrate deep into epithelial tissue (Barkema et al., 2006). For instance, S. aureus has been found in membrane-bound vacuoles inside bovine epithelial cells during a co-culture with the pathogen (Almeida et al., 1996) and within the alveoli and lactiferous ducts of glands collected from dairy cows after 96 hours of a intramammary challenge (Hensen et al., 2000). Remarkably, S. aureus is also capable of colonizing phagocytes and forming biofilm structures inside the gland that further enhance its adherence to epithelial tissues (Mullarky et al., 2001). This ability of contagious pathogens to evade the immune system and colonize the infected gland facilitates the establishment of chronic infections. Cows experimentally infected with $S$. aureus have a limited cytokine and febril response, lower milk somatic cell count (SCC), and less milk yield loss, compared with cows infected with E. coli (Bannerman et al., 2004). In general, mastitis due to contagious pathogens results in a limited immune response and chronic 
infection. In contrast, mastitic infections caused by environmental pathogens are acute and present more severe symptoms.

In contrast to contagious pathogens, the primary sources of environmental pathogens such as E. coli, Streptococcus uberis and Klebsiella pneumonia are the surroundings in which the cow lives (NMC, 1996). Although new infections by these pathogens can occur during milking, environmental pathogens are primarily contracted from the surroundings of the cow such as manure, bedding, and soil. Furthermore, the mammary gland is not a natural habitat for coliform bacteria which are rarely transmitted from infected to uninfected cows (Hogan and Smith, 2003). Coliform mastitis usually occurs without clinical signs but it can cause appearance of abnormal milk, swelling of the gland, decreased appetite, reduced milk yield, dehydration, and diarrhea (Wilson and Gonzalez, 2003). The severity of IMI caused by E. coli can vary from mild to severe which are characterized by systemic responses including rumen stasis, dehydration, and shock (Wenz et al., 2006). Although coliform mastitis can cause endotoxemia and subsequent death, most cases are self-limiting and the loss of milk yield is less severe after recovery (Harmon, 1994). Usually, coliform bacteria such as E. coli can establish an infection without attachment to the mammary gland epithelia (Opdebeeck et al., 1988). Contrary to IMI caused by contagious pathogens, most mastitic infections due to coliform pathogens last for less than a month, result in a robust release of cytokines, and are characterized by more severe systemic responses (Harmon, 1994; Bannerman et al., 2004). 


\section{IMMUNE DYSFUNCTION DURING THE PERIPARTURIENT PERIOD}

Most infectious diseases and metabolic disorders occur during the periparturient period in dairy cows, defined as the last 3 weeks before parturition to 3 weeks after calving (Drackley, 1999). For instance, the number of first cases of clinical mastitis is considerably higher during the dry period and early lactation compared with those detected during the rest of lactation (Smith et al., 1985a,b; Oliver and Sordillo, 1988; Wilson et al., 2004). In addition, periparturient cows challenged with an intramammary infusion of E. coli had more severe clinical signs of mastitis and a more rapid bacterial growth after 10 hours of inoculation, compared with midlactation animals (Shuster et al., 1996). Similarly, intramammary infusion with $100 \mu \mathrm{g}$ of LPS resulted in more severe local and systemic clinical signs of infection in early lactation cows, compared with midlactation animals (Lehtolainen et al., 2003). Although a cause-effect relationship has been difficult to prove, this increased incidence and severity of mastitis during the periparturient period may be the consequence of a state of immune dysfunction (Burvenich et al., 2007). Furthermore, periparturient immune dysfunction does not affect independent components of the immune system. Instead, a wide range of both innate and acquired host defense mechanisms are compromised during this time (Mallard et al., 1998).

During the periparturient period, both innate and adaptive immune systems display defective function. Because migration into the mammary gland and subsequent antimicrobial activity by neutrophils provide the first line of defense against the invading pathogen, extensive research has focused on periparturient alterations in neutrophil function (Paape et al., 2003). First, these phagocytes have reduced ability to sense antigen 
and become activated which can delay their recruitment into the mammary gland. For instance, neutrophils from early lactation cows have lower expression of genes of the TLR4 signaling pathway, involved in the recognition of LPS and subsequent immune activation (Stevens et al., 2011). Second, neutrophils express reduced levels of the adhesion molecules L-selectin (CD62L) and $\beta 2$-integrin (CD18) during the first days after parturition which may impair their ability to migrate through the endothelium and reach the mammary gland (Lee and Kehrli, 1998). Finally, neutrophils have compromised capacity to use their antimicrobial weapons against the invading pathogen, as indicated by numerous tests of neutrophil function which have focused on ROS production, chemotaxis, phagocytosis and bacterial killing.

Numerous groups have consistently reported that neutrophils have impaired generation of ROS during the first 3 or 4 weeks after parturition (Kehrli and Goff, 1989; Kehrli et al., 1989; Cai et al., 1994; Detilleux et al., 1995; Dosogne et al., 1999; Hoeben et al., 2000; Mehrzad et al., 2001a; Revelo and Waldron, 2010; Stevens et al., 2011). This inability of neutrophils to produce adequate amounts of ROS during early lactation may contribute to increased mastitis during the periparturient period. Indeed, low generation of ROS by neutrophils has been associated with increased severity of coliform mastitis in dairy cows (Heyneman et al., 1990). In contrast to the well-documented decrease in neutrophil ROS production postpartum, experiments investigating the changes in chemotaxis during the periparturient period have yielded inconsistent results. For instance, Kehrli et al. (1989) reported an increase in random neutrophil migration during the few weeks before parturition followed by a drastic decrease during the first week after calving. Similarly, Stevens et al. (2011) reported lower neutrophil chemotaxis towards 
complement C5a on day 3 postpartum, compared with day 150 postpartum. However, Cai et al. (1994) and Detilleux et al. (1995) found no changes in directed neutrophil migration during the 5 weeks around parturition in healthy cows. Perhaps, the ability of these cells to perform directed migration decreases as the dairy cow transitions between midlactation into the prepartum period, but does not change considerably around the time of calving. Furthermore, neutrophils from cows with retained placenta have reduced chemotaxis during the first week of parturition (Cai et al., 1994). Thus, it is possible that defects in neutrophil chemotaxis predispose the animals to develop other periparturient complications such as retained placenta, particularly during the first week of lactation (Kimura et al., 2002b).

Numerous researchers have measured the phagocytic ability of neutrophils during the 5 weeks around parturition and have reported either a transient increase between the last week of pregnancy and first week after calving (Guidry et al., 1976; Detilleux et al., 1995; Dosogne et al., 1999) or no change at all in neutrophil phagocytosis during the periparturient period (Cai et al., 1994; Daniel et al., 1991; Dosogne et al., 2001). Detilleux et al. (1995) speculated that the increase in neutrophil phagocytosis at parturition occurs because the cells use the extra energy available for phagocytic processes in a period when the consumption of energy for production of ROS is reduced. Ultimately, the integration of chemotaxis, phagocytosis, and production of antimicrobial compounds defines the killing ability of the neutrophil. In periparturient cows, several groups have reported a decrease in the antimicrobial ability of PMNL during the first 2 weeks after parturition, compared with the few weeks before calving (Kehrli and Goff, 1989; Daniel et al., 1991; Detilleux et al., 1995). However, other investigators have 
reported no changes in the antibody-mediated cytotoxicity of PMNL during the periparturient period in healthy cows (Kehrli et al., 1989; Cai et al., 1994). Alternatively, we reported that the killing capacity of PMNL against S. aureus is higher on day 7 postpartum, compared with the prepartum and midlactation periods (Revelo and Waldron, 2010). Perhaps, differences in methodologies used to determine the antimicrobial capacity of PMNL could explain the inconsistency of results relating to changes in the antimicrobial capacity of PMNL during the periparturient period (Detilleux, 2004).

Changes in lymphocyte population dynamics in blood, milk, and mammary gland tissue occur during the periparturient period and may be related to altered adaptive immune competence (Park et al., 1992). Furthermore, reduced lymphocyte proliferative responses in the periparturient period have been reported by several investigators (Kehrli et al., 1989; Saad et al., 1989; Shafer-Weaver et al., 1996; Nonnecke et al., 2003). In addition to compromised mitogen-stimulated proliferation, the proportion of $\mathrm{T}$ lymphocytes in the gland decreases during periparturition, and lymphocytes collected from the blood of periparturient cows have lower cytotoxic activity, compared with those from midlactation animals (Shafer-Weaver et al., 1996). Further alterations in adaptive immune mechanisms during the periparturient period include reduced lymphocyte secretion of IFN- $\gamma$ (Ishikawa et al., 1994) and IgM (Lacetera et al., 2005), lower IL-2 in mammary gland secretions (Sordillo et al., 1991), and increased monocyte production of TNF- $\alpha$ (Sordillo et al., 1995). Furthermore, Detilleux et al. (1994) reported that the serum IgG concentration in healthy cows decreased to a lowest level on the week before calving but improved after parturition, suggesting that even before colostrogenesis the antibody 
production by B cells is impaired. In summary, these findings indicate that reduced responsiveness of bovine lymphocytes and a lower ability to secrete their products may contribute to the state of immune dysfunction during the periparturient period.

\section{Causes of immune dysfunction}

Altered immune capacity during the periparturient period can not be explained by the fluctuation of a single physiological factor. Instead, the changes in hormones, metabolites, and mineral status that occur to support the physiological adaptations of pregnancy and lactogenesis seem to affect the function of various components of the immune system (Mallard et al., 1998; Goff, 2006). In general, there is a co-incidence between immune dysfunction during the periparturient period, structural changes in the mammary gland, and a metabolic shift from anabolism to catabolism controlled by systemic and local hormones (Vangroenweghe et al., 2005). Numerous metabolic and endocrine factors associated with this adaptation to pregnancy and lactation have been proposed to be causal towards immune dysfunction during the periparturient period.

\section{Metabolic factors}

The metabolism of the dairy cow is considerably challenged during the periparturient period due to increased nutrient requirements to support pregnancy and the onset of milk secretion (Bell, 1995). For instance, the requirement of pregnant cows for glucose on day 21 prepartum is approximately $1,100 \mathrm{~g} / \mathrm{d}$ whereas that of lactating cows has been estimated at 2,500 $\mathrm{g} / \mathrm{d}$ on day 21 postpartum (Overton, 1998). Despite the increased demand of nutrients during the periparturient period, voluntary dry matter intake does not increase at a sufficient rate to meet energetic and nutrient demands of lactation. Therefore, the animal enters a state of negative energy balance and undergoes 
substantial adjustments to cope with the augmented nutrient requirements (Bell and Bauman, 1997). During periods of negative energy balance, stored triacylglycerols (TG) are hydrolyzed by several lipases to yield one molecule of glycerol and free fatty acids which are released into the circulation as non-esterified fatty acids (NEFA). As a result, plasma NEFA concentrations increase approximately $100 \%$ between days 17 and 2 prepartum and $100 \%$ again at parturition (Grummer, 1993). Some of these NEFA can be utilized by the skeletal muscle for energy as this tissue reduces the use of glucose during early lactation. However, because the liver takes up NEFA in proportion to their concentration in blood, increased lipid mobilization results in increased uptake of NEFA by the liver (Drackley, 1999). The fate of these NEFA in the liver includes complete oxidation to carbon dioxide, partial oxidation to acetic acid or ketone bodies, and TG resynthesis for hepatic storage or release as very-low density lipoproteins. Increased amounts of NEFA removed by the liver promote ketogenesis and thus, the release of ketone bodies including $\beta$-hydroxybutyrate (BHBA) into the circulation (Drackley et al., 2001).

Metabolic responses to negative energy balance are thought be one contributor to periparturient immune dysfunction. However, several groups have investigated the effects of experimentally manipulated energy balance on measurements of immune function with only modest success. For instance, Perkins et al. (2001) fed steers at $60 \%$ of their maintenance requirements to induce negative energy balance and reported that neutrophils from these feed-restricted animals had a slight increase in the expression of L-selectin. However, no change in the expression of Mac-1 or MCH molecules were detected when compared with steers fed at $210 \%$ of requirements (Perkins et al., 2001). 
Furthermore, the same authors reported no differences in leukocyte counts, rumen motility, plasma and milk TNF- $\alpha$, and milk IgG concentrations between midlactation cows fed at $80 \%$ of their maintenance requirements, compared with cows fed ad-libitum (Perkins et al., 2002). Recently, Moyes et al. (2009) reported that neutrophils from cows under dietary-induced negative energy balance had a $13 \%$ lower phagocytosis of fluorescent beads immediately before an intra-mammary challenge with Streptococcus uberis, compared with neutrophils from cows in positive energy balance. Conversely, no differences in neutrophil chemotaxis and milk concentrations of TNF- $\alpha$, IL-10 and IL-1 $\beta$ between experimental groups were detected in this study. Together, these findings suggest that experimental negative energy balance due to feed-restriction causes minimal alterations on several measures of immune function.

Experiments in which negative energy balance was induced in dairy cattle have failed to provide a causal link between periparturient metabolic adaptations and immunosuppression. However, several investigators have reported that altered concentration of the metabolites associated with negative energy balance disrupt immune function. Most notably, changes in fatty acid metabolism around parturition including high levels of non-esterified fatty acids (NEFAs) and ketone bodies are likely responsible for impaired immunity (Suriyasathaporn et al., 2000). For instance, Hammon et al. (2006) found a negative correlation $(\mathrm{R}=0.44)$ between plasma NEFA levels and neutrophil myeloperoxidase activity in cows with puerperal metritis and subclinical endometritis. Although this study did not prove a cause-effect relationship between plasma NEFA and neutrophil respiratory burst activity, evidence from in vitro studies indicates that high levels of NEFA impair production of ROS by neutrophils. Incubation of neutrophils with 
high $(2$ and $1 \mathrm{mM})$, moderate $(0.5$ and $0.25 \mathrm{mM})$ or low $(0.125$ and $0.0625 \mathrm{mM})$ concentrations of NEFA resulted in a modest reduction in oxidative burst activity in those cells incubated with low and moderate levels of NEFA (Scalia et al., 2006). In agreement, Ster et al. (2012) reported a dose-dependent reduction in ROS production by neutrophils incubated with $0.25,0.50$, and $0.75 \mathrm{mM}$ of a NEFA mixture containing a fatty acid profile similar to that encountered in bovine serum. In addition to high circulatory NEFA, elevated levels of ketone bodies such as BHBA may disrupt neutrophil function. For instance, Hoeben et al. (1997) reported that 1 and $2.5 \mathrm{mM}$ of BHBA in media inhibited the respiratory burst of neutrophils from healthy periparturient cows by 10 and $15 \%$, respectively. In agreement, Grinberg et al. (2008) found that pre-incubation of neutrophils with increasing levels of BHBA reduced their phagocytosis of E. coli, release of NETs and NET-derived killing of the pathogen, in a dose-dependent manner. In summary, high NEFA and BHBA levels in circulation during the periparturient period may impair the innate immune response by reducing the oxidative burst, phagocytosis, release of NETs, and ultimate killing of bacteria by the neutrophils.

Elevated circulatory concentrations of NEFA and ketone bodies during the periparturient period may also impair the function of cells of the adaptive immune system. For example, adding NEFA to PBMCs cultured in medium containing serum from cows on day 61 postpartum, reduced their proliferative responses and IFN- $\gamma$ secretion to levels similar to those by PBMC incubated with serum from cows on day 5 postpartum (Ster et al., 2012). In addition, the same investigators reported that increasing concentrations of NEFA in culture reduced the proliferation responses of PBMCs in a dose-response manner (Ster et al., 2012). Moreover, Suriyasathaporn et al. (1999) 
reported that leukocytes from cows with high circulatory levels of BHBA (> $1.6 \mathrm{mM})$ have a lower chemotactic activity compared to cells from cows with low and medium BHBA levels ( $<0.8$ and 0.8 to $1.6 \mathrm{mM}$, respectively). Consistently, leukocytes incubated with BHBA, acetoacetate, and acetone at levels similar to those in subclinically and clinically ketotic cows had impaired migration ability, compared with lymphocytes incubated with media alone (Suriyasathaporn et al., 1999). Although the mechanisms by which high NEFA and ketone bodies impair immune cell function during the periparturient period are still under investigation, it is possible that a change in the phospholipid profile of leukocytes towards saturated fatty acids may contribute to the defects in immune cellular function during this period (Contreras et al., 2010).

In addition to changes in the plasma levels of NEFA and ketone bodies, the decrease in circulatory glucose during early lactation has been proposed to cause immune dysfunction during the periparturient period. Although the metabolism and fate of energetic fuels in bovine leukocytes is unclear, the concentration of glucose increases in response to an IMI challenge suggesting that glucose may be an important source of energy for immune cells (Moyes et al., 2009). To test the hypothesis that low glucose levels during early lactation compromise immune function, Nonnecke et al. (1992) measured the secretion of IgM by blood mononuclear cells (MNL) incubated with increasing concentrations of glucose. Although MNL incubated with a glucose level similar to that of ketotic cows had similar IgM secretion compared to controls, these investigators reported a marked decrease in IgM secretion by MNL incubated in the absence or a very low concentration of glucose. In summary, mobilization of NEFA and ketone bodies, as well as hypoglycemia due to negative energy balance during the 
periparturient period have been shown to alter the function of several components of the innate and adaptive immune system in the dairy cow.

The effects of improved energy balance on the immune function of periparturient dairy cows have also been investigated via experimental increase of their feed intake. For instance, Stabel et al. (2003) increased the dietary energy intake of periparturient cows by mechanically stuffing refused feed into fistulated cows with Johne's disease and hypothesized that supplemental energy would prevent the disruption of immune function around parturition. These investigators reported that blood lymphocytes from cows receiving supplemental feed had increased $\operatorname{IgG}$ production but reduced proliferative capacity after stimulation in vitro. In contrast, neutrophil chemotaxis and iodination did not differ between stuffed and control animals indicating that supplemental feeding enhanced lymphocyte antibody production but did not improve leukocyte proliferation and neutrophil function. In addition to feed stuffing, removal of the mammary gland has been used to determine the effects of improved energy balance on immune function via elimination of the metabolic stress due to milk production. Kimura et al. (1999) reported that mastectomized cows have improved neutrophil myeloperoxidase activity and expression of $\beta$-integrin during the postpartum period, compared with intact animals. Similarly, mastectomy prevented the decline in T lymphocytes (helper-, cytotoxic-, and gamma-delta-T lymphocytes) characteristic of periparturient immunosuppression (Kimura et al., 2002a) and improved DNA synthesis, interferon- $\gamma$ (IFN- $\gamma$ ) and IgM production by peripheral blood mononuclear cells (Nonnecke et al., 2003). Interestingly, all intact animals in the study by Kimura et al. (1999) developed milk fever suggesting that low levels of calcium alone or in combination with negative energy balance may 
contribute to altered PMNL function in postpartum dairy cows. In addition to the decrease in plasma calcium during the days around calving, PBMC from healthy and cows with milk fever had decrease release of intracellular calcium in response to stimulation by anti-CD3 and secondary antibodies (Kimura et al., 2006). Together, these findings suggest that elimination of the metabolic insults due to lactogenesis may facilitate the recovery of several components of the innate and adaptive immune function, especially during the postpartum period. Interestingly, low levels of calcium and defects in calcium metabolism in immune cells may contribute to the alteration of immune function during the periparturient period.

\section{Hormonal factors}

The changes in the concentration of hormones and endocrine factors that occur during the transition period likely contribute to periparturient immune dysfunction. For example, extensive research has demonstrated that glucocorticoids exert immunosuppressive effects on several components of the innate and adaptive immune system. Coincident with the surge in plasma cortisol, neutrophils from cows and newborn calves have decreased expression of the leukocyte adhesion molecule L-selectin (CD62L) during the day after calving (Lee and Kehrli, 1998). Consistently, injection of the glucocorticoids cortisol and dexamethasone to cows causes a potent down-regulation of CD62L and $\beta-2$ integrin (CD16) on the surface of neutrophils (Burton et al., 1995). Furthermore, in vitro incubation of neutrophils with glucocorticoids inhibits their respiratory burst activity upon activation with a mitogen (Hoeben et al., 1998). In addition to a potent immunosuppressive effect on neutrophils, glucocorticoid administration alters several components of the adaptive immune system. Injection of 
dexamethasone to dairy bulls results in a reduced number of circulatory $\gamma / \delta$ T-cells, the major subset of T lymphocytes in dairy cows (Burton and Kehrli, 1996). Similarly, Nonnecke et al. (1997) reported that dairy bulls injected with dexamethasone have a lower proportion of circulating B cells, CD3 T cells, and $\gamma / \delta$ T-cells, as well as decreased in vitro secretion of interferon- $\gamma$ and IgM by activated mononuclear cells and B cells, respectively. Therefore, it is clear that glucocorticoids suppress several components of both innate and adaptive branches of the bovine immune system. However, the peak of cortisol that occurs at calving lasts only hours and may not fully explain the prolonged state of immunosuppression observed around parturition, especially during the prepartum period (Overton and Waldron, 2004).

The mechanisms used by the immune system to solve infectious processes are lowest during late pregnancy, parturition, and early lactation suggesting a potential role for sex hormones (Vangroenweghe et al., 2005). In dairy cows, the plasma level of estradiol increases progressively during late pregnancy, peaks several days prior to parturition, and remains low during early lactation whereas progesterone concentrations are high during pregnancy and decrease just before parturition (Bell, 1995). Androgens and estrogens modulate the immune responses in different species and play a key role in the development of certain chronic and autoimmune diseases in humans (Straub, 2007). In dairy cows, however, similar effects have been difficult to establish and efforts to detect receptors for sex hormones have yielded inconsistent results. For instance, Winters et al. (2003) failed to detect estrogen receptors (ER)- $\alpha$ and progesterone receptors on the surface of neutrophils collected from ovariectomized cows using immunohistochemistry. Lamote et al. (2006) reported that blood neutrophils have mRNA encoding for ER- $\alpha$ and 
ER- $\beta$ but only detected ER- $\beta$ at the protein level by flowcytometry. Regardless of the putative presence of the ER on bovine leukocytes, injection of $17 \beta$-estradiol (E2) to dairy cows did not alter neutrophil phagocytosis (Guidry et al., 1975). Also, in vitro incubation of neutrophils with $\mathrm{E} 2$, estrone, or progesterone (P4) did not alter their respiratory burst activity (Winters et al., 20003). Perhaps, sex hormones do not directly modulate the function of immune cells but may influence the systemic inflammatory response to an immune challenge. For example, LPS-challenged steers injected with E2 had increased plasma concentrations of TNF- $\alpha$, xanthine oxidase activity and nitric oxide production whereas animals receiving a progesterone injection had lower levels of nitric oxide production and plasma TNF- $\alpha$, compared with steers injected with a vehicle, suggesting that E2 augments the pro-inflammatory response after LPS, whereas P4 treatment attenuates the same response (Kahl et al., 2011).

Lactogenic hormones such as growth hormone $(\mathrm{GH})$, insulin-like growth factor I (IGF-I), and prolactin regulate the transition from a proliferative to a lactating mammary gland (Bell, 1995). These hormones have been proposed to counteract the immunesuppressive effects of endocrine factors released under stressful conditions such as cortisol (Dorshkind and Horseman, 2001). In humans and animal models, GH and IGF-I regulate the development and function of the immune system via control of hematopoietic and lymphoid tissues (Clark, 1997). For example, GH-deficient children and adults have impaired cytokine production, natural killer activity and neutrophil function (Velkeniers et al., 1998). In dairy cows, several groups have hypothesized that the administration of recombinant bovine somatotropin ( $\mathrm{rbST}$ ) enhances the immune response of the animal. The first line of evidence suggesting that $\mathrm{GH}$ can modulate the 
immune system in dairy cows is the presence of its receptor on the membrane of leukocytes. Bovine PBMCs express mRNA encoding GH receptor (Ohtsuka et al. 2008) whereas IGF-1 receptors have been detected on the cell-membrane of bovine monocytes and neutrophils by flowcytometry (Nielsen et al., 2003). Furthermore, Hoeben et al. (1999a) injected cows with rbST before intramammary challenge with Streptococcus uberis and reported that the content of bacteria in the milk from infected quarters was lower in rbST-treated cows compared with control animals. In addition, rbST-treated cows had less severe general and local clinical symptoms of inflammation after the bacterial challenge (Hoeben, 1999b). These findings suggest that GH administration improves the capacity of the host defense mechanisms to clear an IMI. Additionally, in vivo and in vitro studies indicate that $\mathrm{GH}$ enhances the proliferative responses of activated leukocytes (Burton et al., 1991; Burton et al., 1992b; Elvinger et al., 1991; Hoeben et al., 1999b) and increases the production of IgG, IgG2, and IgA (Burton et al., 1992a). Augmented proliferative responses of leukocytes in response to rbST administration in vivo may be the result of $\mathrm{GH}$ action on the bone marrow which stimulates the release of stored cells and activation of the colony-stimulating factor (Burvenich et al. 1999). Despite the effects of GH on cells of the bovine adaptive immune system being well-documented, the evidence supporting a beneficial effect of $\mathrm{GH}$ on neutrophil antimicrobial function is less conclusive. On one hand, results published in non-peer reviewed publications have indicated that rbST administration in vivo enhanced the respiratory burst and NADPH oxidase activity in blood PMNL (reviewed by Burvenich et al., 1999). Conversely, Elvinger et al. (1991) reported that rbST injection to dairy heifers did not improve neutrophil cytochrome c reduction, 
chemotaxis, and phagocytosis and killing of E. coli. Also, injection of rbST did not improve the neutrophil production of reactive oxygen species (ROS), diapedesis trough epithelial monolayers and expression of the adhesion receptors CD11a and $\mathrm{CDb}$ (Hoeben et al., 1999b). However, it is possible that low statistical power in the studies by Elvinger et al. $(1991, \mathrm{n}=4)$ and Hoeben et al. $(1999 \mathrm{~b}, \mathrm{n}=5)$ resulted in failure to detect a beneficial effect of GH on neutrophils. In summary, exogenous GH may enhance the animal's ability to resolve an intramammary infection via an improved lymphocyte proliferative response and antibody production. In contrast, that GH modulates neutrophil function is to some extend controversial.

Growth hormone release is regulated by hypothalamic secretion of GH releasing hormone $(\mathrm{GHRH})$ and somatostatin. Interestingly, plasmid mediated gene transfer technology has been used to test the effects of GHRH on immune parameters in pregnant dairy heifers (Brown et al., 2004). These researchers administered a myogenic GHRHexpressing plasmid by intramuscular injection, followed by electroporation to accomplish relevant levels of plasmid DNA transfer, and collected blood samples 18 and 300 days after initiation of treatments. Injection of the GHRH plasmid resulted in higher total number of T-cell subpopulations $\left(\mathrm{CD} 2^{+}, \mathrm{CD} 25^{+} \mathrm{CD} 4^{+}\right.$and $\left.\mathrm{CD} 4^{+} \mathrm{CD} 45 \mathrm{R}^{+}\right)$by day 18 and higher naïve lymphocytes, natural killer cells by day 300, compared with control heifers. In addition, GHRH-treated heifers had improved body condition scores, decreased mortality and fewer cases of hoof pathologies. Although, these findings highlight the potential for long-term stable production of endogenous hormones using plasmid technology, it is difficult to determine whether the effects of GHRH treatment on these 
animals were mediated by GH or IGF-1 as the authors did not report their levels in circulation.

The effects of GH on tissues can be elicited either directly or through the action of insulin-like growth factor I (IGF-I). Although the role of IGF-1 on bovine immune responses is unclear, injection of rbST to dairy cows results in a marked increase in plasma concentration of IGF-1 (Bauman, 1992). Therefore, it is likely that the enhanced host response to infection in cows treated by rbST may be partially mediated by IGF-1. In humans, IGF-1 stimulates the phagocytic activity, complement receptor expression, and oxidative burst activity of neutrophils collected from the blood of healthy patients (Bjerknes and Aarskog, 1995). In dairy cows, however, only indirect evidence of this potential effect exists. Burton et al. (1992b) reported a positive association between IGF1 plasma concentrations and total number and percentage of PMN in circulation, and a negative relationship between plasma IGF-1 and the number and percentage of lymphocytes and monocytes. These findings, in combination with evidence of IGF-1 receptors in leukocytes provided by Nielsen et al. (2003), warranted further investigation on the effects of IGF-1 on leukocytes. Recently, Sander et al. (2011) tested the hypothesis that changes in plasma IGF-1 during the periparturient period can account for the altered neutrophil function during this time. These investigators failed to find a correlation between plasma IGF-1 and phagocytic function of neutrophils. Alternatively, IGF-1 may have a role in immune cellular homeostasis by increasing the longevity of the leukocytes in the mammary gland (Vangroenweghe et al., 2005). However, there is no clear evidence that IGF-1 plays an important role during immune dysfunction of periparturient dairy cows. 
The increase in plasma prolactin (PRL) and prolactin receptors in the mammary gland that occurs with the onset of lactation are essential for the initiation of lactogenesis. For instance, PRL has direct effects on ion transport, amino acid transport, golgi volume, and synthesis and secretion of milk fat and proteins (Tucker, 2000). In addition, prolactin may regulate the metabolic adaptations of transition cows, supporting the partitioning of nutrients towards the mammary gland (Bauman and Currie, 1980). Furthermore, PRL may play an important role in maintaining normal immune function, especially during periods of physiological challenges including pregnancy and lactation (Dorshkind and Horseman, 2001). In dairy steers, Auchtung and Dahl (2004) reported that PRL treatment in vitro stimulates the proliferation of lymphocytes collected from animals exposed to short day photoperiods (SDPP) but did not alter the proliferation of lymphocytes from steers on long day photoperiods (LDPP). In the same study (Auchtung and Dahl, 2004), animals on SDPP had reduced plasma PRL but increased expression of PRL receptor on peripheral blood mononuclear cells, compared with those on LDPP. These investigators concluded that SDPP may increase the sensitivity of leukocytes to PRL enhancing the ability of PRL to stimulate cellular immune function. Indeed, dairy cows exposed to SDPP during the dry period have neutrophils with improved chemotaxis and lymphocytes with enhanced cellular proliferation, compared with animals on LDPP (Auchtung et al., 2004). Together, these findings suggest that photoperiod influences immune function via PRL and altered PRL signaling during periods of stress, may impair not only immune function, but also mammary growth and lipid metabolism (Dahl, 2008).

In summary, numerous changes in the circulating concentrations of metabolites during the periparturient period seem to influence the immune response of dairy cows. 
Remarkably, the increases in circulating levels of NEFA and ketone bodies during this period are the best candidates to explain the causes of immune dysfunction. In addition, numerous hormonal and endocrine factors have been proposed to modulate the immune function of dairy cows during the transition period including glucocorticosteroids, sex hormones, GH, IGF-1, and PRL.

\section{NUTRITIONAL APPROACHES TO IMPROVE IMMUNITY}

\section{Nutritional strategies to improve immunity via metabolic health}

As discussed in previous sections, several metabolic and endocrine factors associated with negative energy and calcium balances likely contribute to immune dysfunction in periparturient cows. Therefore, strategies aimed to minimize the excessive mobilization of adipose tissue in the form of NEFA and ketone bodies, as well as improve calcium metabolism will likely improve immune health during the transition period (Overton and Waldron, 2004).

Current nutritional management recommendations by the NRC (2001) include feeding dairy cows a diet containing 1.25 McalNEL/kg DM during the far-off period but increasing the energy content of the close-up diet to 1.62 McalNEL/kg DM. Providing excessive energy to dairy cows in the early dry period should be avoided as it may have negative carryover effects on postpartum metabolic health. For instance, Dann et al. (2006) reported that feeding cows a diet containing energy at $160 \%$ of NRC recommendations during the far-off period deteriorated the postpartum energy balance, compared with cows fed at 77 and $95 \%$ of energy requirements. In addition, this excessive energy during the far-off period increased circulatory BHBA, decreased DMI, 
and tended to augment plasma NEFA concentrations (Dann et al., 2006). Given that disproportionate lipid mobilization during the periparturient period may contribute to immune dysfunction via increased circulatory NEFA and BHBA, grouping and nutritional strategies to avoid excessive energy intake during the far-off period while providing sufficient nutrients during the close-up period may indirectly benefit the immune competence of the periparturient dairy cow.

Awareness of the benefits of omega-3 (n-3) fatty acids on human health has generated interest in the potential use of individual n-3 fatty acids to improve energy metabolism in dairy cows. To determine their effects on lipid metabolism, Mashek et al. (2005) infused one of three isocaloric lipid emulsions into the circulation of fasted cows: an emulsion containing $20 \%$ of TG from tallow to mimic the fatty acid composition found in ruminants, linseed oil with a high content of linolenic acid, and fish oil with high contents of eicosapentaenoic acid (EPA) and docosahexaenoic acid (DHA). These authors reported that the infusion of linseed oil resulted in decreased concentrations of plasma TG, NEFA, and BHBA as well as decreased TG in liver, compared with tallow and fish oil emulsions. In addition to experiments in which n-3 fatty acids were infused into the cow to avoid rumen biohydrogenation, feeding n-3 FA has been shown to improve energy metabolism in transition cows. Cows fed a diet supplemented with extruded flaxseed that provided 161 and $376 \mathrm{~g}$ of linolenic acid during the last 30 days prepartum and the first 10 days postpartum, respectively, had an improved energy balance, a 3.8\% increase in DMI postpartum, and a $6.4 \%$ increase in milk yield, compared with cows fed a isocaloric-isonitrogenous diet supplemented with ether extract (Zachut et al., 2010). Similarly, Petit et al. (2007) fed no lipid supplement, whole 
flaxseed high in linolenic acid or a calcium salt high in saturated fatty acids to dairy cows from 6 days prepartum through day 28 postpartum. Cows fed the flaxseed had increased concentrations of glycogen in liver postpartum, compared to both control and calcium salt FA-supplemented cows, suggesting that feeding supplemental linolenic acid can be used as a strategy to increase liver concentrations of glycogen in the transition cow. Together, these findings suggest that omega-3 FA, especially linolenic acid, may reduce hyperlipidemia and TG accumulation in liver during the periparturient period. Such effects can be particularly favorable to decrease the massive mobilization of lipids and therefore improve immune function.

A beneficial-carryover effect of restricting DMI in the dry period on postpartum metabolism and lactation has been proposed. Although homogenous restriction of feed intake may be unpractical in group-feeding systems, restricting the intake of dairy cows during the prepartum period to approximately $80 \%$, increases postpartum DMI and milk yield, and decreased plasma NEFA (reviewed by Overton and Waldron, 2004). In a recent study, Hayirli et al. (2011) restricted the intake of dairy cows by $26 \%$ on as fed basis during the last 4 weeks of pregnancy and reported that fed-restricted cows had higher postpartum DMI, lost less body weight, improved energy balance, and tended to have lower plasma NEFA during the postpartum period than cows fed ad-libitum. Therefore, feed restriction may also minimize the impact of increased NEFA on immune function during the periparturient period. However, feed restriction may be unviable to implement in group feeding systems as feed restriction has been found to increase competition for feed at the bunk and compromise uniform cow access to their feed (Collings et al., 2011). 
In addition to prevent hypocalcemia, appropriate calcium nutrition may contribute to minimize immune dysfunction during the periparturient period. One of the underlying causes of hypocalcemia in periparturient dairy cows is metabolic alkalosis. As a result of this condition, several process are impaired including tissue responsiveness to parathyroid hormone, calcium mobilization from bones, and production of 1,25 dihydroxyvitamin D3 needed for calcium transport in the intestine (Horst et al., 1997). To prevent metabolic alkalosis, the cation-anion difference or CAD $\left[\left(\mathrm{Na}^{+}+\mathrm{K}^{+}\right)-\left(\mathrm{Cl}^{-}+\right.\right.$ $\left.\mathrm{S}^{-2}\right)$ ] in the diet can be adjusted by either adding anions or reducing cations in the diet. In general, prepartum diets with a low CAD increase the tissue response to parathyroid hormone and improve the adaptation of the dairy cow to the increased requirement of calcium for milk synthesis (NRC, 2001). However, one of the challenges to decrease the $\mathrm{CAD}$ in the prepartum diets is the difficulty to formulate diets low in potassium or to add suitable anions to the diet. To overcome this problem prepartum diets low in calcium can be formulated in order to stimulate calcium homeostatic mechanisms prior to parturition. Another route to reduce dietary calcium is the addition of sodium aluminum silicate (zeolite A) to the rations which binds dietary calcium and reduces its absorption (Goff, 2006).

\section{Dietary nutrients that improve immune function in periparturient dairy cows}

Inadequate amounts of dietary vitamins and trace minerals with antioxidant functions may be one of the underlying causes of periparturient immunosuppression (Goff, 2006; Spears and Weiss, 2008). Dairy cows experience higher oxidative stress during the transition period as indicated by higher circulatory thiobarbituric acid-reactive substances (TBARS) and reactive oxygen metabolites (ROM) immediately before and 
following parturition (Bernabucci et al., 2005). When these oxidant molecules are not effectively removed by the antioxidant system, oxidative stress may compromise the health of the dairy cow (Miller et al., 1993). For instance, oxidative stress may impair the protective functions of the immune cells via increased lipid peroxidation of polyunsaturated fatty acids and reduced membrane fluidity (Bendich, 1993). In addition, excessive generation of oxygen radicals by activated phagocytes may cause mammary epithelial cell damage and compromise its function (Lauzon et al., 2005; Zhao and Lacasse, 2008). Therefore, the protective role by antioxidant mechanisms is particularly essential during the periparturient period when the dairy cow experiences augmented oxidative stress (Sordillo et al., 2007).

Dietary antioxidants can support the immune health of dairy cows by enhancing host antioxidant defenses, especially during the periparturient period (Sordillo et al., 2009). Dietary antioxidants can be divided into two groups depending on their mode of action. The first group scavenges oxidants directly and includes micronutrients such as vitamins $\mathrm{E}$ and $\mathrm{C}$, and $\beta$-carotene. The second group includes the minerals $\mathrm{Zn}, \mathrm{Cu}, \mathrm{Mn}$, $\mathrm{Se}$, and Fe which do not act directly as scavengers but are components and cofactors of enzymes involved in the antioxidant defense system (Bendich, 1993). In addition to these traditional roles as antioxidants, trace minerals such as $\mathrm{Zn}$ can influence immune cell function directly via regulation of gene expression, hematopoiesis and cytokine production (Haase and Rink, 2009; Prasad, 2007).

Although interactions with numerous dietary factors may reduce the bioavailability of trace minerals as inorganic salts (e.g. sulfates, oxides, and carbonates), supplementation of trace minerals to dairy cows has traditionally relied on inorganic 
sources (Andrieu, 2008). However, trace minerals from organic sources resist antagonism by dietary factors and thus are more bioavailable compared with minerals from inorganic sources (Predieri et al., 2005; Wedekind et al., 1992). In dairy cows, replacement of Mn, $\mathrm{Cu}$, and $\mathrm{Zn}$, and $\mathrm{Co}$ from inorganic sources with equivalent amounts of these trace minerals as organic complexes has increased milk yield (Ballantine et al., 2002; Hackbart et al., 2010; Nocek et al., 2006) and milk fat content (Formigoni et al., 2011; SicilianoJones et al., 2008). In addition, trace mineral supplementation from organic sources improved the content of total Igs content in colostrum (Formigoni et al., 2011), and decreased the incidence of sole ulcers (Siciliano-Jones et al., 2008) suggesting that a more bioavailable form of these trace minerals may also improve immune health. However, little research has been conducted to examine the effects of replacing trace minerals from inorganic sources with equivalent amounts of trace minerals as organic complexes on cellular immunity such as neutrophil function in dairy cows.

In addition to promoting enhanced immunity via their antioxidant properties, several micronutrients such as B-vitamins have immune-modulating properties. Bvitamins are a group of water soluble vitamins involved in numerous aspects of immune function through their involvement in cellular metabolism, nucleic acid, and protein biosynthesis. In humans, deficiencies in $\mathrm{B}_{6}, \mathrm{~B}_{12}$, and folate impair several components of the innate and adaptive immune systems including lymphocyte growth and proliferation, natural killer cell activity, and cytokine production (reviewed by Wintergerst et al., 2007). In dairy cows, the requirements for B-vitamins have been estimated based on lactating sow needs adjusted for body weight and milk production, however, the amounts of B-vitamins required to support gestation, milk production and health have not been 
established (NRC, 2001). Moreover, the concept that rumen microorganisms are capable of synthesizing significant amounts of B-vitamins has led to the dogma that ruminal synthesis and escape of dietary sources are sufficient to meet the requirements of dairy cows (NRC, 2001). Whether B-vitamin supplementation boosts the immune function of dairy cows, in particular during the periparturient period, warrants investigation.

Omega-3 fatty acids can also modulate the immune and inflammatory responses in a direct manner. Individual n-3 fatty acids regulate gene expression, serve as precursors for the synthesis of bioactive lipid mediators, and modify the fluidity and raft structure of cell membranes (Calder 2008). However, only a limited number of studies have been conducted to investigate the direct effect of omega-3 fatty acids on the immune system of dairy cows. Lessard et al. (2003) reported that dairy cows fed flaxseed (43.9\% C18:3n-3, 25.6\% C18:2n-6) during the first 100 days of lactation had increased serum n3 fatty acids, but no change on the ex vivo lymphocyte production of IFN, prostaglandin E2 (PGE2), and antibody production against ovalamin, compared with cows fed isonitrogenous, isoenergetic, and isolipidic supplements based on calcium salts of palm oil, and micronized soybeans. Recently, Sun et al. (2010) infused 0, 100, 200, 300 and $400 \mathrm{~g} /$ day of $\alpha$-linolenic acid (ALA) into the duodenum of midlactation cows for one week to determine its effects on serum concentrations of $\operatorname{IgG}, \operatorname{IgA}$, IgM, IFN, IL-2, IL-4, IL-6, IL-10 and PGE2. These investigators reported that doses between 100 and 300 g/day of ALA increased plasma IgG and IFN, decreased IL-4 and PGE2, and had no effect on the concentrations of IgA, IgM, IL-2, IL-6, and IL-10. Although these studies suggest that omega-3 fatty acids have immunomodulatory effects, further research is 
needed to determine whether these fatty acids can be fed to enhance the host resistance to infection during the periparturient period.

\section{CONCLUSION}

In dairy cows, most infectious diseases and metabolic disorders occur during the periparturient period when the animals experience increased demands for nutrients to support pregnancy and the onset of lactation (Drackley, 1999). Particularly, the incidence and severity of clinical mastitis increase during this time in part due to incompetent immune and antioxidant systems (Mallard et al., 1998; Sordillo and Aitken, 2009). Neutrophils are a key component of the innate immune system and the dominant cell type in the infected gland. In fact, the resolution of clinical mastitis depends mainly on the ability of these cells to migrate into the mammary gland, phagocytize and use their antimicrobial weapons to eliminate the pathogen (Vangroenweghe et al., 2005). In addition, neutrophils may have a significant role in the innate immune response against Gram-negative bacteria via recognition of LPS and the production of inflammatory mediators (Sohn et al., 2007b; Worku and Morris, 2009). However, little is known about the direct effects of LPS on the mechanisms used by bovine neutrophils to eliminate pathogens. Therefore, an initial study was conducted to better understand the responses of blood neutrophils to E. coli LPS. In Chapter Two, it was hypothesized that a short term incubation of neutrophils with LPS would stimulate their ROS generation, NET release, chemotaxis, killing capacity against S. aurous, and expression of genes encoding for cytokines and proteins involved in antimicrobial function. Additionally it was expected 
that the expression of these genes would be reduced in neutrophils collected from early lactation, compared to those from midlactation cows.

Several lines of research indicate that neutrophils have a reduced ability to detect the infectious agent, become activated, cross the endothelial cell lining, migrate to the mammary gland, and ultimately use their numerous antimicrobial mechanisms against the invading pathogen. Although this neutrophil dysfunction may not be explained by the alteration of a single physiological factor, inadequate amounts of dietary vitamins, trace minerals, and antioxidants may be one of the underlying causes of periparturient immunosuppression (Goff, 2006). Therefore, in Chapter Three, it was hypothesized that feeding cows a dietary supplement containing immunomodulatory nutrients such as Bvitamins, dehydrated yeast, and Trichoderma longibrachiatum fermentation products would have enhanced generation of ROS, release of NETs, chemotaxis, and antimicrobial ability of blood neutrophils. In Chapter Four, RNA next generation sequencing technology was used to discover the differential gene expression in neutrophils collected from cows fed this dietary supplement. Given that the effects of dietary trace minerals from organic sources on neutrophil function has not been investigated in periparturient dairy cows, in Chapter Five, it was hypothesized that cows supplemented with amino acid complexes of $\mathrm{Mn}, \mathrm{Cu}$, and $\mathrm{Zn}$, and $\mathrm{Co}$ glucoheptonate would have enhanced neutrophil function, relative to animals supplemented with the same amount of trace minerals from inorganic sources. Finally, in Chapter Six, RNA-sequencing experiments were conducted to determine the effects of supplementation with trace minerals from organic sources on the genetic profile of neutrophils. The findings from these experiments will increase our understanding of the biology of neutrophils during immune 
dysfunction and offer nutritional strategies to improve the immune competence of periparturient dairy cows. 


\title{
CHAPTER TWO
}

\section{IN VITRO EFFECTS OF ESCHERICHIA COLI LIPOPOLYSACCHARIDE ON THE FUNCTION AND GENE EXPRESSION OF NEUTROPHILS ISOLATED FROM THE BLOOD OF DAIRY COWS}

\begin{abstract}
The objectives of this study were to investigate the effect of Escherichia coli lipopolysaccharide (LPS) on the function of bovine neutrophils (PMNL) collected from midlactation cows and determine the differential effects of LPS on gene expression of PMNL purified from early and midlactation cows. Neutrophils from midlactation cows (187 \pm 13 d postpartum) were incubated with $0,1,25$ and $50 \mu \mathrm{g} / \mathrm{mL}$ of LPS for 120 min and the generation of reactive oxygen species (ROS), PMNL extracellular traps (NETs), chemotaxis and killing of Staphylococcus aureus were determined. Incubation of PMNL with $25 \mu \mathrm{g} / \mathrm{mL}$ of LPS increased intracellular ROS by $79 \%$ in mitogen-stimulated PMNL. Fifty $\mu \mathrm{g} / \mathrm{mL}$ of LPS enhanced intracellular ROS by non-stimulated and stimulated PMNL by 184 and 154\%, respectively. Non-stimulated PMNL incubated with 25 and $50 \mu \mathrm{g} / \mathrm{mL}$ of LPS had a $105 \%$ increase in NETs. LPS had no effect on subsequent PMNL chemotaxis or killing of $S$. aureus. To examine the effect of LPS on the expression of genes involved in PMNL function and cytokine production, mRNA was purified from PMNL isolated from midlactation (146 \pm 2 postpartum; $n=10)$ and early lactation cows ( 7 d postpartum; $\mathrm{n}=10$ ), after a 120 -min incubation with 0 or $50 \mu \mathrm{g} / \mathrm{mL}$ of LPS. Amounts of interleukin-8 (IL-8), tumor necrosis factor (TNF), bactericidal/permeability-increasing protein (BPI), myeloperoxidase (MPO), superoxide
\end{abstract}


dismutase 2 (SOD2), NADPH oxidase 4 (NOX4), cytochrome b-245, alpha polypeptide (CYBA), histone H2A/1 (H2A/1) and histone H2B-like (H2B) mRNA were determined relative to $\beta$-actin by real-time quantitative PCR. Regardless of stage of lactation, PMNL incubated with $50 \mu \mathrm{g} / \mathrm{mL}$ of LPS had 537 and $45 \%$ higher mRNA contents of IL-8 and SOD2 compared with $0 \mu \mathrm{g} / \mathrm{mL}$ LPS, respectively. Also, LPS augmented the expression of TNF, BPI and CYBA (2908, 59 and 158\% compared with controls, respectively) only in PMNL from midlactation cows. LPS did not affect mRNA levels of MPO, NOX4, H2A/1 and H2B. Independent of LPS treatment, PMNL from midlactation cows had 99\% higher mRNA contents of IL-8, compared with PMNL from early lactation cows. PMNL from early lactation had a $634 \%$ increase in MPO mRNA expression, relative to PMNL from midlactation cows. These results suggest that LPS directly stimulates PMNL to produce ROS and express NETs. In addition, LPS enhances the generation of ROS by PMNL in response to other stimuli and increases the expression of genes encoding inflammatory mediators and enzymes involved in the production of ROS. Finally, reduced PMNL gene expression of IL-8 (regardless of LPS activation), TNF, CYBA, and BPI (upon stimulation with LPS) in early lactation may elucidate several mechanisms by which PMNL may become immune-incompetent during this period. 


\section{INTRODUCTION}

During intramammary infections (IMI) with Gram-negative bacteria, growth and death of the invading pathogen results in the shedding of the lipopolysaccharide components (LPS) of the bacterial cell wall (Burvenich et al., 2003). Lipopolysaccharide has a core oligosaccharide, repeated polysaccharide units (O-antigens) and a lipid A domain, which causes the toxic effects of LPS (Hogan and Smith, 2003). In the mammary gland, LPS initiates the inflammatory response in part by stimulating macrophages and monocytes to secrete pro-inflammatory cytokines into the surrounding environment (Paape et al., 2002). In response to this stimulus, circulating polymorphonuclear leukocytes (PMNL or neutrophils) are rapidly recruited to the mammary gland. As an example, infusion of $100 \mu \mathrm{g}$ of Escherichia coli LPS into the quarters of healthy midlactation cows reduced the number of circulating PMNL by approximately $75 \%$, and resulted in a dramatic influx of somatic cells into the mammary gland as early as 4 hours after the challenge (Bannerman et al., 2003). In the milk of LPS challenged quarters, PMNL are the predominant cellular population whereas macrophages and monocytes comprise the majority of leukocytes present in the healthy mammary gland (Saad and Ostensson, 1990). The successful migration of PMNL into the mammary gland via chemotaxis and subsequent phagocytosis and killing are crucial for the resolution of IMI (Vangroenweghe et al., 2005). Bovine PMNL use several strategies to destroy the invading pathogen including microbial uptake, intracellular killing and the secretion of antimicrobial molecules (e.g. reactive oxygen species or ROS) to the extracellular space (Paape et al., 2003). Neutrophils are also capable of releasing granule proteins and de-condensed chromatin to form extracellular fibers known as neutrophil 
extracellular traps (NETs) that bind microorganisms, preventing them from distributing throughout the tissue, and killing the pathogens by keeping them in a high local concentration of antimicrobial agents (Brinkmann et al., 2004). The function of bovine PMNL is altered during the periparturient period (Kehrli et al., 1989; Cai et al., 1994; Revelo and Waldron, 2010) contributing to the attenuated pathogen killing capacity observed after calving (Dosogne et al., 2001).

Large amounts of LPS can be released in the infected mammary glands of cows with naturally occurring coliform mastitis (Anri, 1989; Hakogi et al., 1989). Absorption of LPS into the general circulation has been reported 6 to $8 \mathrm{hrs}$ after intramammary infusion of $10 \mathrm{mg}$ of $E$. coli LPS (Ziv et al., 1976) and in cows with naturally occurring Gram-negative bacterial mastitis (Hakogi et al., 1989). Mammary macrophages and epithelial cells are presumed to recognize LPS to produce cytokines that initiate the inflammatory response (Burvenich et al., 2007). These cytokines include chemoattractants that recruit circulating PMNL to the infected gland where the phagocytes engulf and destroy invading pathogens. However, PMNL may have secondary but important roles in the innate immune response against Gram-negative bacteria. These roles include recognition of LPS and the production of inflammatory mediators including interleukin-1 $\beta$ (IL-1 $\beta$ ), interleukin-12 (IL-12), interferon (IFN)gamma, interleukin-8 (IL-8), and tumor necrosis factor- $\alpha$ (TNF- $\alpha$ ) (Sohn et al, 2007b; Worku and Morris, 2009). Mammary macrophages and epithelial cells recognize and bind LPS using their toll-like receptors (TLRs) such as TLR-4 assisted by the accessory molecules LPS binding protein (LBP), cluster of differentiation 14 (CD14) and myeloid differentiation protein 2 (MD-2; De Schepper et al., 2008; Ibeagha-Awemu et al., 2008). 
Bovine PMNL use a similar mechanism to recognize LPS since they express TLR-4 on their cell surface (Worku and Morris, 2009) and are capable of shedding membrane CD14 upon in vitro exposure to E. coli LPS (Sohn et al., 2007a).

Lipopolysaccharide may have a direct effect on the activation of PMNL in response to the invading pathogen. For example, incubation of human PMNL with LPS increases their oxidative burst (Forehand et al., 1989; Aida and Pabst, 1990; Böhmer et al., 1992) and phagocytosis of Staphylococcus aureus (Böhmer et al., 1992). In mice, culture of bone marrow PMNL with LPS induces the intracellular ROS-dependent activation of nuclear factor $\kappa \mathrm{B}(\mathrm{NF}-\mathrm{\kappa B})$, a transcription factor that subsequently regulates the gene expression of many pro-inflammatory cytokines (Asehnoune et al., 2004). In cows, increased ROS generation by PMNL has been reported after incubation of blood PMNL with $P$. haemolytica LPS (McClenahan et al., 2000) and intrammamary infusion of E. coli LPS (Mehrzad et al., 2001a). However, in the study by Mehrzad et al. (2001a), it is likely that the increase in ROS and modulation of cell viability were influenced by other inflammatory mediators and cytokines rather than just LPS alone. Previous research suggests that LPS per se induces the secretion of cytokines and inflammatory mediators by bovine PMNL (Sohn et al, 2007b; Worku and Morris, 2009). Whether LPS directly influences the mechanisms used by bovine PMNL to eliminate pathogens has not been investigated. Therefore, the objective of this study was to determine the effects of LPS on the functional capacity and gene expression of PMNL isolated from the blood of dairy cows. We hypothesized that: i) a short term incubation of PMNL with LPS would alter their ROS generation, NET release, chemotaxis, and killing capacity against S. aureus, ii) LPS would stimulate the PMNL expression of genes encoding for cytokines and proteins 
involved in antimicrobial function, and iii) expression of these genes would be reduced in PMNL from early lactation, compared to midlactation cows.

\section{MATERIALS AND METHODS}

\section{Blood Donors}

The use and care of all animals were approved by The University of Missouri Animal Care and Use Committee. Only multiparous Holstein cows entering second or greater lactation were used in this study. All cows were free of obvious clinical infection or inflammation, their rectal temperatures were less than $39.5^{\circ} \mathrm{C}$ and current $\mathrm{SCC}$ less than 200,000 cells $/ \mathrm{mL}$. To determine the effects of LPS on the function of neutrophils, 60 $\mathrm{mL}$ of blood were collected from seven cows in midlactation $(187 \pm 13$ days after

calving). To investigate the differential effects of LPS on the gene expression of neutrophils from cows relative to their stage of lactation, $120 \mathrm{~mL}$ of blood were collected from 10 cows in midlactation (146 \pm 2 days after calving) and 10 cows in early lactation (7 days after calving). Blood was collected via coccygeal vessel puncture into evacuated tubes containing acid-citrate dextrose (Vacutainer ${ }^{\circledR}$, Becton Dickinson, Franklin Lakes, $\mathrm{NJ})$, inverted three times and stored on ice until laboratory processing (<20 min). All functional assays were performed simultaneously on the same day for any given cow.

\section{Reagents}

Percoll $^{\circledR}$ was obtained from GE Healthcare Bio-Sciences Corp. (Piscataway, NJ). Lipopolysaccharide from E. coli serotype O55:B5 (strain CDC 1644-70; conjugated to fluorescein isothiocyanate) was obtained from Sigma Chemical Co. (St. Louis, MO). Trizol $^{\circledR}$ was acquired from Invitrogen Co. (Carlsbad, CA). Luminol (5-amino-2,3- 
dihydro-1,4-phthalazinedione; Sigma Chemical Co.) and phorbol 12-myristate,13-acetate (PMA; Sigma Chemical Co.) were prepared as $89.4 \mathrm{~m} M$ and $162.12 \mu M$ stock solutions, respectively, in dimethyl sulfoxide (DMSO). The 5-(6)-chloromethyl-2',7'dichlorodihydrofluorescein diacetate acetyl ester (CM- $\mathrm{H}_{2}$ DCFDA; Invitrogen Co.) was prepared as $1,730.7 \mu M$ stock solution in DMSO. Sytox ${ }^{\circledR}$ Orange nucleic acid stain was obtained from Invitrogen Co. as a $5 \mathrm{~m} M$ solution in DMSO. All stock solutions were stored at $-20^{\circ} \mathrm{C}$. All working solutions, cell suspensions and reactions were prepared using endotoxin-free, calcium and magnesium-free Hanks' Balanced Salt Solution (CMFHBSS; Sigma Chemical Co.) or RPMI-1640 (GIBCO, Grand Island, NY) + 2\% heatinactivated FBS and were pre-warmed to $37^{\circ} \mathrm{C}$ immediately prior to their use in the assays. Calcium and magnesium free HBSS, and RPMI-1640 were used in our in vitro cellular system to maintain a balanced environment (intra- and extracellular osmolarity, $\mathrm{pH},\left[\mathrm{O}_{2}\right]$, and temperature) and provide the cells with water and inorganic ions essential for normal cell metabolism. The use of HBSS as buffering medium during PMNLchemiluminescence assays results in maximal light emission and cell activation compared to PBS and Tris (Freitas et al., 2009).

\section{Isolation of Bovine Neutrophils}

Neutrophils were isolated using procedures modified from Weber et al. (2001) as validated in our laboratory. Blood $(10 \mathrm{~mL})$ was aliquotted into $50-\mathrm{mL}$ polypropylene conical tubes (Corning Inc., NY) and centrifuged at $1,000 \times g$ for 20 min at $4^{\circ} \mathrm{C}$ to separate plasma from the cell pack. Plasma was discarded and the remaining cells were suspended in $35 \mathrm{~mL}$ of CMF-HBSS. The suspension was gently pipetted down the side of a $50-\mathrm{mL}$ polypropylene conical tube containing $10 \mathrm{~mL}$ of $1.084 \mathrm{~g} / \mathrm{mL}$ of Percoll ${ }^{\circledR}$. The 
cells were then subjected to gradient centrifugation $\left(400 \mathrm{x} g\right.$ for $40 \mathrm{~min}$ at $\left.22^{\circ} \mathrm{C}\right)$. The supernatant, mononuclear cell layer and Percoll ${ }^{\circledR}$ were aseptically aspirated and discarded. The pellet (PMNL and erythrocytes) was retained and erythrocytes were lysed by hypotonic shock. For this procedure, two volumes of an ice-cold $10.56 \mathrm{mM} \mathrm{Na} 2 \mathrm{HPO}_{4}$, $2.67 \mathrm{mM} \mathrm{NaH}{ }_{2} \mathrm{PO}_{4}$ solution were added to one volume of cell suspension and the solution was mixed by gentle aspiration and inversion. Tonicity was restored by the addition of one volume of a $10.56 \mathrm{~m} M \mathrm{Na}_{2} \mathrm{HPO}_{4}, 2.67 \mathrm{~m} M \mathrm{NaH}_{2} \mathrm{PO}_{4} 0.43 M \mathrm{NaCl}$ solution. The cell solution was then centrifuged $(850 \mathrm{x} g)$ for $4 \mathrm{~min}$ at $4^{\circ} \mathrm{C}$ and the pellet washed twice by resuspension in $35 \mathrm{~mL}$ of CMF-HBSS and recentrifugation (850 x $g$ for 4 min at $4^{\circ} \mathrm{C}$ ). Cells were re-suspended in CMF-HBSS (luminol, CM- $\mathrm{H}_{2}$ DCFDA, chemotaxis and killing assays) or RPMI-1640 + 2\% heat-inactivated FBS (NET assay). Neutrophils were then enumerated and viability was determined by propidium iodide (Sigma Chemical Co.) exclusion using a Cellometer Vision ${ }^{\circledR}$ automatic cell counter (Nexcelom Bioscience LLC., Lawrence, MA). The concentration of cells was adjusted for every assay and the suspensions were kept at $4^{\circ} \mathrm{C}$ until incubation with LPS.

\section{LPS Incubations}

To investigate the effects of LPS on PMNL function, we incubated blood PMNL with $0,1,25$ and $50 \mu \mathrm{g} / \mathrm{mL}$ of $E$. coli $\mathrm{LPS}$ for $120 \mathrm{~min}$. Similar doses $(0,1,10$ and 100 $\mu \mathrm{g} / \mathrm{mL}$ ) have been used to study the effect of LPS on the bovine PMNL production of TNF- $\alpha$, IL-1 $\beta$, IL-12 and IFN- $\gamma$ in vitro (Sohn et al., 2007b), and are within the range of doses often used during experimental intramammary LPS challenges. For the functional assays, working solutions of LPS in CMF-HBSS (luminol, CM- $\mathrm{H}_{2}$ DCFDA, chemotaxis and killing assays) or RPMI-1640 + 2\% heat-inactivated FBS (NET assay) were pipetted 
into polystyrene round-bottomed tubes (Becton Dickinson Labware, Franklin Lakes, NJ) containing $5 \times 10^{6}$ cells $/ \mathrm{mL}$ (luminol and CM- $\mathrm{H}_{2}$ DCFDA assays), $3.75 \times 10^{6}$ cells $/ \mathrm{mL}$ (chemotaxis assay), $6.25 \times 10^{6}$ cells $/ \mathrm{mL}$ (NET assay) and $2 \times 10^{5}$ cells $/ \mathrm{mL}$ (killing assay) to reach final concentrations of $0,1,25$ or $50 \mu \mathrm{g} / \mathrm{mL}$ of LPS. The tubes were then incubated with lids in a humidified incubator $\left(37^{\circ} \mathrm{C}, 5 \% \mathrm{CO}_{2}\right)$ for 120 minutes while inverted in a Labquake ${ }^{\circledR}$ tube rotator (Barnstead-Thermolyne Corp., Dubuque, IA). After incubation, the cell suspensions were mixed by aspiration and the functional assays performed. To determine the effect of LPS on gene expression, a working solution of 250 $\mu \mathrm{g} / \mathrm{mL}$ of LPS was added to $50-\mathrm{mL}$ polypropylene conical tubes (Corning Inc., NY) containing purified neutrophils to reach final concentrations of 0 or $50 \mu \mathrm{g} / \mathrm{mL}$ of LPS at a cell concentration of $6 \times 10^{6}$ cells $/ \mathrm{mL}$. The tubes were then placed in a humidified incubator $\left(37^{\circ} \mathrm{C}, 5 \% \mathrm{CO}_{2}\right)$ and incubated for 120 minutes while inverted in a Labquake ${ }^{\circledR}$ tube rotator. After incubation, the cell solutions were centrifuged $(850 \mathrm{x} g)$ for $4 \mathrm{~min}$ at $4^{\circ} \mathrm{C}$, the pellet resuspended in $\operatorname{Trizol}^{\circledR}$ at a concentration of $1 \times 10^{7}$ cells $/ \mathrm{mL}$ and stored at $-80^{\circ} \mathrm{C}$ until mRNA extraction.

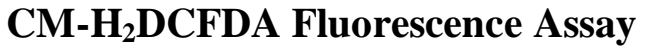

An index of the intracellular neutrophil ROS production was assessed using a CM- $\mathrm{H}_{2}$ DCFDA fluorescence assay (Revelo and Waldron, 2010). One hundred microliters of cell suspension were pipetted into the wells $\left(5 \times 10^{5}\right.$ cells/well) of whitewalled, clear-bottomed 96-well microplates (Corning Inc., NY). All reactions were carried out in sextuplicate. Ten $\mu \mathrm{L}$ of $200 \mu M \mathrm{CM}-\mathrm{H}_{2}$ DCFDA and $30 \mu \mathrm{L}$ of either 400 nM PMA (stimulated cells) or HBSS (non-stimulated cells) were added to all wells. All reactions were then adjusted to $200 \mu \mathrm{L}$ using CMF-HBSS. Fluorescence was measured 
every 5 min for $240 \mathrm{~min}$ with a Synergy HT plate reader at an excitation wavelength of $485 \mathrm{~nm}$ and an emission wavelength of $528 \mathrm{~nm}$. Background values, defined as the mean fluorescence values of CM- $\mathrm{H}_{2}$ DCFDA diluted in CMF-HBSS were subtracted from all readings.

\section{Luminol Chemiluminescence Assay}

After incubation with LPS, an index of the total neutrophil ROS production was immediately assessed by a luminol chemiluminescence assay as described by Revelo and Waldron (2010). Fifty microliters of cell suspension were pipetted into the wells $(2.5 \mathrm{x}$ $10^{5}$ cells/well) of white-walled, clear-bottomed 96-well microplates (Corning Inc., NY). All reactions were carried out in sextuplicate. Twenty $\mu \mathrm{L}$ of luminol and $20 \mu \mathrm{L}$ of either 400 nM PMA (stimulated cells) or HBSS (non-stimulated cells) were added to all wells. All reactions were then adjusted to $200 \mu \mathrm{L}$ using CMF-HBSS. Chemiluminescence (CL) was measured every $5 \mathrm{~min}$ for $240 \mathrm{~min}$ with a Synergy HT plate reader (BioTek Instruments, Inc., Winooski, VT). Background values, defined as the mean CL values of luminol diluted in CMF-HBSS, were automatically subtracted from all readings.

\section{Neutrophil Extracellular Trap (NET) Assay}

Neutrophil extracellular trap formation was quantified using Sytox ${ }^{\circledR}$ Orange as described by Revelo and Waldron (2010). After PMNL incubation with LPS, $100 \mu \mathrm{L}$ of cell suspension were pipetted into the wells $\left(6.25 \times 10^{5}\right.$ cells/well $)$ of white-walled, clearbottomed 96-well microplates (Costar Inc., NY). All reactions were performed in sextuplicate. Twenty microliters of PMA and $20 \mu \mathrm{L}$ of ionomycin (in RPMI-1640 + 2\% heat-inactivated FBS) to obtain a final concentration of $16.21 \mathrm{nM}$ and $1 \mu M$, respectively, were added to the stimulated set of wells. Forty microliters of RPMI-1640 $+2 \%$ heat- 
inactivated FBS were added to the control wells. The plates were then centrifuged (10 min at $650 \times g)$ and incubated $\left(37{ }^{\circ} \mathrm{C}\right.$ and $\left.5 \% \mathrm{CO}_{2}\right)$ for $60 \mathrm{~min}$. After incubation, supernatant from the plates was removed, and the wells were stained and kept protected from light with $50 \mu 1$ of $5 \mu M$ Sytox Orange in CMF-HBSS (15 min at room temperature). The stain was poured off, and the wells washed three times with $100 \mu 1$ of CMF-HBSS. A Synergy HT plate reader was used to detect fluorescence with an excitation wavelength of $530 \mathrm{~nm}$ and emission wavelength of $580 \mathrm{~nm}$. Background values, defined as the mean fluorescence values of PMA and ionomycin diluted in RPMI1640 were subtracted from all readings.

\section{Chemotaxis Assay}

Chemotaxis was performed in a 48-well micro chemotaxis chamber (Neuro Probe Inc., Gaithersburg, MD) according to the manufacturer's recommendations. The wells in the bottom plate of the chamber were filled with $26 \mu \mathrm{L}$ of the chemoattractant interleukin 8 (IL-8; $100 \mathrm{ng} / \mathrm{mL}$ ) or CMF-HBSS, previously warmed to $37^{\circ} \mathrm{C}$. A slight positive meniscus was formed when the wells were filled with the chemoattractant. A 25 x $80 \mathrm{~mm}$ polycarbonate filter (non- polyvinylpyrrolidone treated, Neuro Probe Inc.) was lowered onto the formed meniscus, allowing the middle portion of the filter to make contact first. The polycarbonate filter had pores with a diameter of $5 \mu \mathrm{m}$ to allow migration and adhesion of PMNL to the underside of the filter where they could be stained and counted. A silicone gasket was then applied on top of the filter and the top component of the micro chemotaxis chamber fastened to the bottom chamber. The chamber was pre-warmed for $10 \mathrm{~min}$ at $37^{\circ} \mathrm{C}$ before adding the PMNL to the upper chamber. Fifty-five $\mu 1$ of cell suspensions $\left(3.75 \times 10^{6}\right.$ neutrophils/ml) previously incubated with increasing 
concentrations of LPS were added to the top chamber and incubated at $37^{\circ} \mathrm{C}$ in humidified air with $5 \% \mathrm{CO}_{2}$ for 30 min to allow the PMNL to migrate towards the chemoattractant. After the incubation, the fluid from the top chamber was aspirated and the chamber disassembled. The side of the filter membrane that had faced the upper chamber was rinsed with CMF-HBSS and wiped off three times with a wiper blade to remove non-migrated PMNL. Migrated PMNL were adhered to the side of the filter that had faced the IL-8 (chemoattractant) or CMF-HBSS (control). The filter was immersed in methanol for $10 \mathrm{~min}$, air-dried and stained in a 3.75\% Giemsa staining solution (Sigma Chemical Co.) for $1 \mathrm{hr}$. Subsequently, the membrane was rinsed in Giemsa buffer and placed with the cell-side up on a $50 \times 75 \mathrm{~mm}$ microscope slide for drying. The number of PMNL that migrated through the filter was counted under oil immersion on a light microscope by counting the number of neutrophils present in five microscope fields at $1,000 \mathrm{x}$. Each sample was run in duplicate. Results are reported as the average number of migrated neutrophils towards IL-8 or CMF-HBSS observed per five fields.

\section{Killing Assay}

The killing assay was performed as described by Revelo and Waldron (2010). Staphylococcus aureus Newbould 305 (American Type Culture Collection, Manassas, VA) was inoculated on a blood agar plate (Northeast Laboratory Services Inc., Winslow, $\mathrm{ME}$ ) and incubated overnight at $37^{\circ} \mathrm{C}$. Ten colonies were transferred from the plate to a sterile tube containing $10 \mathrm{~mL}$ of brain-heart infusion broth (Sigma Chemical Co.) and incubated overnight at $37^{\circ} \mathrm{C}$ at $225 \mathrm{rpm}$ in an orbital shaker. After the overnight incubation, the tube was sonicated to disassociate individual bacterial cells using a sonic dismembrator (Sonifier ${ }^{\circledR}$, Branson Ultrasonics Corp., Danbury, CT) and maintained at 
$4^{\circ} \mathrm{C}$ to stop bacterial growth. One hundred microliters from this culture were serially diluted in CMF-HBSS and $100 \mu \mathrm{L}$ of the resulting dilutions were spread on blood agar plates. The stock culture was maintained at $4^{\circ} \mathrm{C}$ and the plates were incubated overnight at $37^{\circ} \mathrm{C}$ to determine the concentration [colony forming units $(\mathrm{cfu}) / \mathrm{mL}$ ] of the stock culture based on the colony counts of the spread plates. Immediately before the initiation of the killing assay, the stock culture was placed in ice and diluted in HBSS to yield a final concentration of $1.6 \times 10^{8} \mathrm{cfu} / \mathrm{mL}$. To assess PMNL killing, $200 \mu \mathrm{L}$ of $S$. aureus ( $3.2 \times 10^{7} \mathrm{cfu}$ ) and $400 \mu \mathrm{L}$ of pooled, heat-inactivated bovine serum were added to sterile tubes with or without $2 \times 10^{6}$ PMNL previously incubated with the different doses of LPS $(1.4 \mathrm{~mL})$. The ratio of bacteria to PMNL was 16:1. All reactions were performed in triplicate. The samples were placed on a Labquake ${ }^{\circledR}$ tube rotator for $60 \mathrm{~min}$ at $37^{\circ} \mathrm{C}$. These reactions were then placed in ice and sonicated with a sonic dismembrator for $15 \mathrm{~s}$ at amplitude of $20 \%$. Rupture of the PMNL was verified by microscopic examination. Sonication does not affect bacterial viability (Rinaldi et al., 2006; Revelo and Waldron, unpublished observation). A $100 \mu \mathrm{L}$ aliquot from each sonicated reaction was serially diluted in CMF-HBSS and $100 \mu \mathrm{L}$ quantities of the resulting dilutions was spread on blood agar plates. The plates were incubated overnight at $37^{\circ} \mathrm{C}$ and the colonies enumerated. The percentage of bacteria killed were determined by calculating the difference in the number of bacteria incubated in the absence and presence of PMNL and dividing this difference by the number of colonies when bacteria were incubated in the absence of PMNL. 


\section{RNA Isolation from PMNL and Reverse Transcription}

Total cellular RNA was isolated from neutrophils purified from $120 \mathrm{~mL}$ of blood using $1 \mathrm{~mL}$ of Trizol $^{\circledR}$ reagent per $7.5 \times 10^{6}$ cells, according to the manufacturer recommendations. The concentration and purity of RNA was determined with a ND-1000 NanoDrop ${ }^{\circledR}$ spectrophotometer (NanoDrop Technologies, Wilmington, DE). Genomic DNA was removed by DNase digestion using the Turbo ${ }^{\mathrm{TM}}$ DNase kit (Applied Biosystems/Ambion, Foster City, CA). The integrity of RNA was assessed by gel electrophoresis $(0.8 \%$ agarose gel in $0.09 M$ Tris-borate and $0.002 M$ EDTA buffer with $0.5 \mu \mathrm{g} / \mathrm{mL}$ ethidium bromide) and the RNA was stored at $-80^{\circ} \mathrm{C}$. Reverse transcription of $400 \mathrm{ng}$ of total cellular RNA to cDNA was performed using the High Capacity cDNA Reverse Transcription kit (Applied Biosystems).

\section{Target Genes, Primer Sets and RT-PCR}

The amount of $\beta$-actin (ACTB), IL-8, TNF, bactericidal/permeability-increasing protein (BPI), myeloperoxidase (MPO), superoxide dismutase 2 (SOD2), NADPH oxidase 4 (NOX4), cytochrome b-245 alpha polypeptide (CYBA), histone H2A.1 transcript variant $1(\mathrm{H} 2 \mathrm{~A} / 1)$ and histone $\mathrm{H} 2 \mathrm{~B}$-like $(\mathrm{H} 2 \mathrm{~B})$ mRNA in neutrophils after a 120 -min incubation with 0 or $50 \mu \mathrm{g} / \mathrm{mL}$ of LPS were measured by real-time quantitative PCR (RT-PCR). The main biological functions of these genes are indicated in Table 2.1. 
Table 2.1. Gene description, symbol and relevant biological functions or processes

\begin{tabular}{|c|c|c|}
\hline Symbol & Description & Biological functions or processes ${ }^{1,2}$ \\
\hline ACTB & $\beta$-Actin & $\begin{array}{l}\text { Structural component of cytoskeleton, nucleotide } \\
\text { and ATP }\end{array}$ \\
\hline IL-8 & Interleukin 8 & $\begin{array}{l}\text { Neutrophil activation-chemotaxis, cell adhesion } \\
\text { regulation }\end{array}$ \\
\hline $\mathrm{TNF}$ & Tumor necrosis factor & $\begin{array}{l}\text { Cytokine activity, transcription } \\
\text { suppressor, inflammatory response, leukocyte } \\
\text { tethering or rolling }\end{array}$ \\
\hline BPI & $\begin{array}{l}\text { Bactericidal/permeability- } \\
\text { increasing protein }\end{array}$ & $\begin{array}{l}\text { Lipopolysaccharide binding, response } \\
\text { bacterium, regulation of cytokine production }\end{array}$ \\
\hline MPO & Myeloperoxidase & Mitochondrial protein with peroxidase activity \\
\hline SOD2 & Superoxide dismutase 2 & $\begin{array}{l}\text { Mitochondrial protein with superoxide dismutase } \\
\text { and oxidoreductase activity }\end{array}$ \\
\hline NOX4 & NADPH oxidase 4 & $\begin{array}{l}\text { Membrane bound, NADPH oxidase activity, } \\
\text { oxido-reductase activity, reactive oxygen species } \\
\text { metabolism }\end{array}$ \\
\hline CYBA & $\begin{array}{l}\text { Cytochrome b- } 245 \text {, alpha } \\
\text { polypeptide }\end{array}$ & $\begin{array}{l}\text { Cytochrome complex assembly, respiratory burst, } \\
\text { superoxide anion generation }\end{array}$ \\
\hline $\mathrm{H} 2 \mathrm{~A} / 1$ & $\begin{array}{l}\text { Histone } \mathrm{H} 2 \mathrm{~A} .1 \text {, transcript } \\
\text { variant } 1\end{array}$ & $\begin{array}{l}\text { Maintains nucleosome structural integrity, } \\
\text { chromatin condensation and binding of chromatin } \\
\text { associated proteins }\end{array}$ \\
\hline $\mathrm{H} 2 \mathrm{~B}$ & Histone H2B-like & $\begin{array}{l}\text { Maintains nucleosome structural integrity, } \\
\text { chromatin condensation and binding of chromatin } \\
\text { associated proteins }\end{array}$ \\
\hline
\end{tabular}

${ }^{\mathrm{T}}$ National Center for Biotechnology Information (NCBI).

${ }^{2}$ Entrez Gene, NCBI. 
Primer sets were designed using PrimerQuest ${ }^{\mathrm{SM}}$ (Integrated DNA Technologies, Coralville, IA) based on publicly available bovine mRNA sequences by the National Center of Biotechnology Information (NCBI; National Center of Biotechnology Information, 2010). RT-PCR products from the primer sets were sequenced by the University of Missouri DNA Core and these sequences aligned against NCBI sequences to verify the amplification of genes of interest using the Basic Local Alignment Search Tool (http://blast.ncbi.nlm.nih.gov/; NCBI, 2010). Features of the primers used in RTPCR are reported in Table 2.2 RT-PCR reactions $(10 \mu \mathrm{L})$ were performed using Power SYBR® Green PCR Master Mix (Applied Biosystems) with $1 \mu M$ concentrations of forward and reverse primers and $1 \mu \mathrm{L}$ of cDNA sample. Reactions included a no template control (NTC) and were performed in triplicates. The SYBR green fluorescence was quantified by the ABI Prism 7500 Sequence Detector (Applied Biosystems). Machine settings consisted of an initial temperature of $50^{\circ} \mathrm{C}$ for $2 \mathrm{~min}$, a polymerase activation temperature of $95^{\circ} \mathrm{C}$ for 10 min followed by 40 PCR cycles consisting of two stages: 1) melting at $95^{\circ} \mathrm{C}$ for $15 \mathrm{sec}$ and 2) annealing and extension at $60^{\circ} \mathrm{C}$ for $1 \mathrm{~min}$. Analysis of amplification plots were performed by the Sequence Detection Software (Applied Biosystems). One plate was used for all cDNA samples for each primer set. Changes in gene expression relative to the reference gene ACTB were calculated by the $2^{\text {(-Delta Delta }}$ ${ }^{\mathrm{C}(\mathrm{T}))}$ method using the mean cycle threshold $(\mathrm{CT})$ values obtained from all RT-PCR experiments (Livak and Schmittgen, 2001). Relative fold changes in gene expression were assessed by calculating the difference between the CT values of ACTB and that of the target gene $(\Delta \mathrm{CT})$. Expression of ACTB was not affected by LPS treatment or stage of lactation $(P>0.20)$. To obtain $\Delta \Delta \mathrm{CT}$ values, the mean $\Delta \mathrm{CT}$ of non-activated PMNL 
from early lactation cows was used as the calibrator for samples from early lactation cows. In consistency, the mean $\Delta \mathrm{CT}$ of non-activated PMNL from midlactation cows was used as the calibrator for samples from midlactation cows. Fold changes in the expression of target genes normalized to ACTB and relative to non-activated PMNL within the same physiological state were calculated by the equation $2^{-\Delta \Delta \mathrm{CT}}$. These values were used in the statistical analysis. 
Table 2.2. Accession number, primer sequences and percentage of similarity between amplified RT-PCR products and publicly available sequences by the National Center for Biotechnology Information (NCBI)

\begin{tabular}{|c|c|c|c|}
\hline Symbol & Accession no. & Primers ( $5^{\prime}$ to $\left.3^{\prime}\right)$ & $\%$ Similar $^{1}$ \\
\hline ACTB & AY141970.1 & $\begin{array}{l}\text { F 5'-AGATGTGGATCAGCAAGCAGGAGT-3', } \\
\text { R 5'-AGCTAACAGTCCGCCTAGAAGCAT-3' }\end{array}$ & 96 \\
\hline IL-8 & NM_173925.2 & $\begin{array}{l}\text { F 5'-GGTGCAGAAGGTTGTGCAGGTATT-3' } \\
\text { R 5'-ACACCAGACCCACACAGAACATGA-3' }\end{array}$ & 97 \\
\hline $\mathrm{TNF}$ & NM_173966.2 & $\begin{array}{l}\text { F 5'-TCAACTCTCCCTTCCTGCCAATGT-3' } \\
\text { R 5'-AGTTTGTGTCTCCCAGGACACCTT-3' }\end{array}$ & 98 \\
\hline BPI & NM_173895.2 & $\begin{array}{l}\text { F 5'-AACCTTTCTGTGGTTGTTGGTGCC-3' } \\
\text { R 5'-TGATGACAGACTGCAGCGACTCAA-3' }\end{array}$ & 96 \\
\hline MPO & NM_001113298.1 & $\begin{array}{l}\text { F 5'-TCGATACCAACCTATGCAGCCCAA-3' } \\
\text { R 5'-ATTTGGTTCTGGCGGTTCAGCTTC-3' }\end{array}$ & 97 \\
\hline SOD2 & NM_174034.2 & $\begin{array}{l}\text { F 5'-TTGCTGGAAGCCATCAAACGTGAC-3' } \\
\text { R 5'-AATCTGTAAGCGTCCCTGCTCCTT-3' }\end{array}$ & 100 \\
\hline NOX4 & AY354499.1 & $\begin{array}{l}\text { F 5'-ACTCTGCTGGATGACTGGAAACCA-3', } \\
\text { R 5'-AGAGTAAGTCTGCAAACCAGCGGA-3' }\end{array}$ & 96 \\
\hline CYBA & NM_174034.2 & $\begin{array}{l}\text { F 5'-TGGCGTCCGGTCTAATCCTTATCA-3' } \\
\text { R 5'-ACCACTCTGGTCAGGTACTTCTGT-3' }\end{array}$ & 97 \\
\hline $\mathrm{H} 2 \mathrm{~A} / 1$ & XM_583411.4 & $\begin{array}{l}\text { F 5'-TCACCATAAGGCCAAGGGCAAGTA-3' } \\
\text { R 5'-ACACTCTGGATCGAGGCATCTCTT-3' }\end{array}$ & 99 \\
\hline $\mathrm{H} 2 \mathrm{~B}$ & NM_001114854.1 & $\begin{array}{l}\text { F 5'-CGCATTACAACAAGCCCTCGACTA-3', } \\
\text { R 5'-AGCTGGTATACTTGGTGACAGCCT-3' }\end{array}$ & 91 \\
\hline
\end{tabular}




\section{Statistical Analysis for the Neutrophil Functional Assays}

The experiment was conducted as a completely randomized design with cow as the experimental unit. Data were analyzed by analysis of variance (ANOVA) as a mixed model using the mixed procedure of SAS (2001). Two mixed models were used for the luminol- and $\mathrm{CM}-\mathrm{H}_{2}$ DCFDA-based assays involving multiple measurements of CL or fluorescence over time. One included the repeated statement for assessment of the consecutive LPS and LPS by time relative to cell-activation interaction effects (Littell et al., 1998). The repeated statement used time as the variable with cow within treatment as the subject and a compound symmetry covariance structure. The second mixed model used calculated areas under the curves generated using the least squares estimates for CL or fluorescence at each time point. Areas under the curves (AUC) were calculated from plotted data points for each experimental condition using the expand procedure of SAS with cubic spline interpolation and the trapezoid rule (SAS, 2001).

The variables in the model statements included LPS treatment, cell activation/inhibition (luminol, CM- $\mathrm{H}_{2}$ DCFDA and NET assays), time (luminol and CM$\mathrm{H}_{2}$ DCFDA assays), chemoattractant (chemotaxis) and all interactions. Least squares means and standard errors were generated using the LSMeans statement in conjunction with the pdiff option of SAS (2001). Tukey's multiple comparison test was used to assess

specific treatment differences for those variables shown to have significant overall treatment effects in the analysis of variance. All reported means are the adjusted least squares means \pm standard error of the mean (SEM) and the significance was declared when $P<0.05$. 


\section{Statistical Analysis for Quantitative RT-PCR}

The analysis of relative fold changes in the expression of target genes was conducted as a completely randomized split-plot design with cow as the experimental unit. Cows were divided according to their stage of lactation into early lactation or midlactation groups. Further, PMNL from each cow were randomly assigned to in vitro incubations with either 0 or $50 \mu \mathrm{g} / \mathrm{mL}$ of LPS. Extreme observations were detected using PROC REG of SAS (2001) and removed from the data set if they presented at least three of the following characteristics: standardized residual $\left(\mathrm{r}_{\mathrm{i}}\right)>2$ standard deviations, high leverage $\left(h_{i i} \geq 2 \mathrm{pn}^{-1}\right.$, where $\mathrm{n}=$ number of observations and $\mathrm{p}=$ number of parameters), possible undue influence [DFITTS $S_{\mathrm{i}} \geq 2\left(\sqrt{p n}^{-1}\right)^{-1}$, DFBETAS $\left._{\mathrm{i}} \geq 2\left(\sqrt{n}_{\mathrm{n}}\right)^{-1}\right]$, cook's distance $\left(\mathrm{D}_{\mathrm{i}}\right)>1$ and ratio of generalized variances $\left(\right.$ COVRATIO $\left._{\mathrm{i}}\right)<1-3 \mathrm{pn}^{-1}$ or $>1+3 \mathrm{pn}^{-1}$ (Kaps and Lamberson, 2004). The distribution of all gene expression data were examined using the Capability procedure of SAS (2001). Relative fold changes data from all genes failed the goodness-of-fit tests for normality $(P<0.05)$. However, the data fit a lognormal distribution $(P>0.05)$. Therefore, gene expression data were analyzed by ANOVA as a generalized linear mixed model implemented in PROC GLIMMIX of SAS (2001) which allows for non-normally distributed data without prior transformation. The variables in the model statements included LPS treatment, stage of lactation and their interaction. The lognormal distribution was specified in the model statement using the DIST and LINK functions of SAS. Least squares means and standard errors were generated using the LSMeans statement in conjunction with the pdiff option of SAS. Tukey's multiple comparison test was used to assess specific treatment differences for those variables shown to have significant overall treatment effects in the analysis of 
variance. For analyses of variance in which the main or interaction effects were significant but Tukey's multiple comparison test revealed no differences between means, orthogonal contrast analysis was used to detect specific treatment differences. All reported means are the adjusted least squares means \pm SEM on the logarithmic scale. Significance was declared when $P<0.05$. The percent changes in gene expression in response to LPS treatment or stage of lactation were calculated by dividing the difference between final and initial by the initial relative gene expression multiplied by 100 . These percentages were calculated using raw units obtained after back-transformation of the least squares means in the logarithmic scale.

\section{RESULTS}

\section{Intracellular Reactive Oxygen Species Production}

Changes in $\mathrm{CM}-\mathrm{H}_{2}$ DCFDA-derived fluorescence, an index of the PMNL intracellular generation of ROS, were observed 15 min after the initiation of the assay (Figure 2.1A). The CM- $\mathrm{H}_{2}$ DCFDA-derived fluorescence from PMA-activated PMNL incubated with 25 and $50 \mu \mathrm{g} / \mathrm{mL}$ of $E$. coli LPS was clearly elevated compared with that of PMNL treated with 0 and $1 \mu \mathrm{g} / \mathrm{mL}$ as early as 25 min after cell activation with PMA. All treatments continued to increase until 240 min after cell activation (time effect, $P<$

0.001). At the end of the assay ( $\min 240$ ), the $\mathrm{CM}-\mathrm{H}_{2} \mathrm{DCFDA}$-derived fluorescence of PMA-activated PMNL incubated with 25 and $50 \mu \mathrm{g} / \mathrm{mL}$ LPS increased by 63 and $118 \%$ compared with the fluorescence of PMNL treated with $0 \mu \mathrm{g} / \mathrm{mL}$ of LPS (Figure 2.1A; LPS effect, $P<0.001$; LPS by time effect, $P<0.001$; LPS by time by cell activation effect $P<0.001)$. In non-activated PMNL, the $\mathrm{CM}-\mathrm{H}_{2}$ DCFDA-derived fluorescence from 
cells incubated with 25 and $50 \mu \mathrm{g} / \mathrm{mL}$ of $E$. coli LPS initiated to increase compared with that of PMNL treated with 0 and $1 \mu \mathrm{g} / \mathrm{mL} 40$ min after the initiation of the assay. At the end of the assay (min 240), the CM- $\mathrm{H}_{2}$ DCFDA-derived fluorescence of non-activated PMNL incubated with 25 and $50 \mu \mathrm{g} / \mathrm{mL}$ LPS increased by 82 and $167 \%$ compared with the fluorescence of non-activated PMNL treated with $0 \mu \mathrm{g} / \mathrm{mL}$ of LPS (Figure 2.1A; LPS effect, $P<0.001$; LPS by time effect, $P<0.001)$. No difference was observed between the $\mathrm{CM}-\mathrm{H}_{2}$ DCFDA-derived fluorescence of PMNL treated with 0 and $1 \mu \mathrm{g} / \mathrm{mL}$ of LPS in non-activated and PMA-activated PMNL.

Areas under the curves were generated from $\mathrm{CM}-\mathrm{H}_{2}$ DCFDA-derived fluorescence plotted data points for each LPS dose and cell activation status (Figure 2.1B). Activation of PMNL with PMA augmented the AUC calculated from CM- $\mathrm{H}_{2}$ DCFDA-derived fluorescence values, regardless of LPS concentration (cell activation effect, $P<0.001$ ). Lipopolysaccharide increased the $\mathrm{CM}-\mathrm{H}_{2}$ DCFDA-derived fluorescence (AUC) in nonactivated and PMA-activated PMNL (Figure 2.1B; LPS effect, $P<0.001$, LPS by cell activation, $P<0.001)$. In non-activated PMNL, $25 \mu \mathrm{g} / \mathrm{mL}$ of LPS tended to increase the AUC relative to $0 \mu \mathrm{g} / \mathrm{mL}$ LPS ( $88 \%$ increase, $P=0.064$ ). Fifty $\mu \mathrm{g} / \mathrm{mL}$ of LPS increased the CM- $\mathrm{H}_{2}$ DCFDA-derived fluorescence (AUC) by $184 \%$ compared with $0 \mu \mathrm{g} / \mathrm{mL}(P<$ 0.001). No difference in $\mathrm{CM}-\mathrm{H}_{2}$ DCFDA-derived fluorescence (AUC) was detected between 0 and $1 \mu \mathrm{g} / \mathrm{mL}$ of LPS ( $P=1.000)$. In PMA-activated PMNL, 25 and $50 \mu \mathrm{g} / \mathrm{mL}$ of LPS increased the CM- $\mathrm{H}_{2}$ DCFDA-derived fluorescence (AUC) compared with 0 $\mu \mathrm{g} / \mathrm{mL}$ of LPS (79 and 154\%, respectively; $P<0.001$ ). In contrast, no difference was detected between the CM- $\mathrm{H}_{2}$ DCFDA-derived fluorescence (AUC) of PMA-activated PMNL treated with 0 and $1 \mu \mathrm{g} / \mathrm{mL}$ of LPS $(P=0.999)$. 
Figure 2.1. Effect of Escherichia coli lipopolysaccharide (LPS) on an index of the intracellular reactive oxygen species (ROS) production by bovine neutrophils (PMNL). PMNL were collected from the blood of cows in midlactation $(n=7)$ and incubated with $0,1,25$ or $50 \mu \mathrm{g} / \mathrm{mL}$ of LPS for $120 \mathrm{~min}$. Subsequently, the 5-(6)-chloromethyl-2',7'dichlorodihydrofluorescein di-acetate acetyl ester (CM-H2DCFDA)-derived fluorescence was measured every 5 min over a 240 min period after addition of phorbol 12myristate,13-acetate (400 nM; PMA-activated PMNL) or HBSS (non-activated PMNL). Fluorescence values of wells without PMNL (containing HBSS and reagents only) were subtracted from fluorescence measurements of wells with PMA-activted and nonactivated PMNL at every time point. (A) CM-H2DCFDA-derived fluorescence in arbitrary units (AU) from non-activated and PMA-activated PMNL measured every 5 min over a 240 min period. Analysis of variance with a repeated measures design was used to detect differences between treatments (LPS effect, $\mathrm{P}<0.001$; time effect, $\mathrm{P}<$ 0.001; LPS by time interaction effect, $\mathrm{P}<0.001$, LPS by time by cell activation effect, $\mathrm{P}$ $<0.001$ ). (B) Areas under the curves (AUC) calculated from plotted CM-H2DCFDAderived measurements for non-activated and PMA-activated PMNL. Twenty five and 50 $\mu \mathrm{g} / \mathrm{mL}$ of LPS increased the total ROS production by PMA-activated PMNL (LPS effect, $\mathrm{P}<0.001$; activation effect, $\mathrm{P}<0.001$; LPS by activation effect, $\mathrm{P}<0.001$ ). Error bars

represent the standard error of the mean. *Bars with an asterisk differ from $0 \mu \mathrm{g} / \mathrm{mL}$ of LPS within the same activation status at $\mathrm{P}<0.05$ (Tukey). \#Bars with a number sign differ from non-activated PMNL within the same LPS dose at $\mathrm{P}<0.05$ (Tukey). 


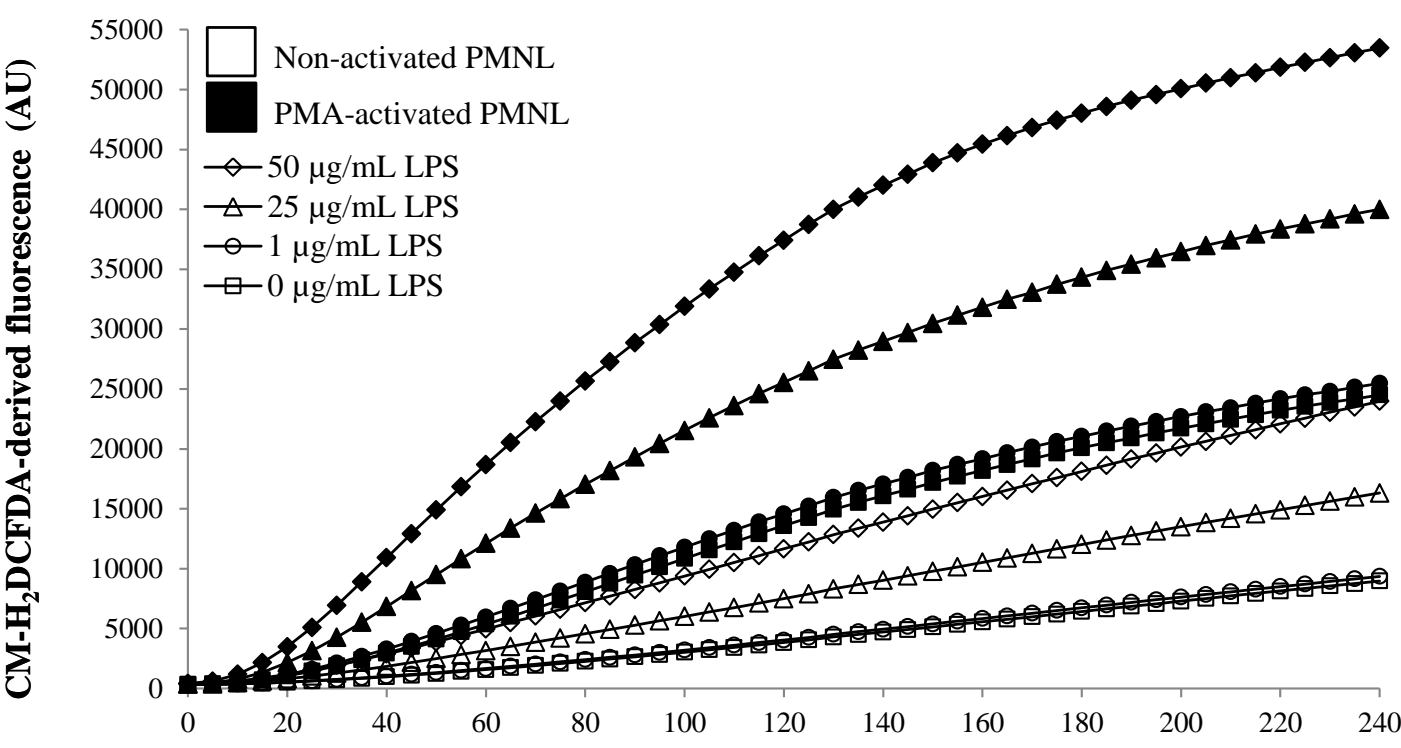

Time relative to activation, $\min$

B

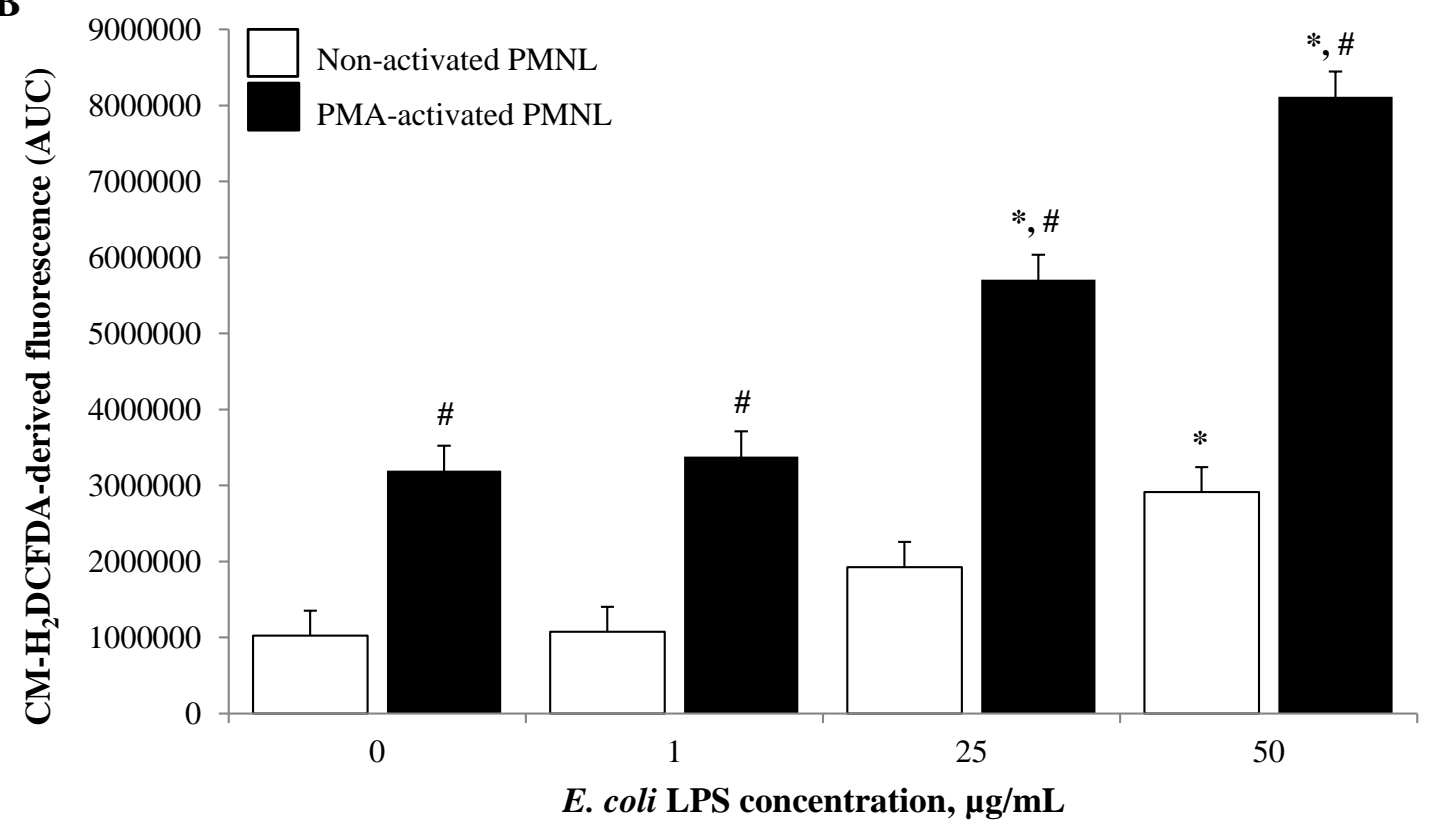




\section{Total Reactive Oxygen Species Production}

In PMA-activated PMNL, luminol-derived CL started to increase $10 \mathrm{~min}$ after cell activation, continued to augment to reach a peak between 75 and 80 min after stimulation and slightly declined afterwards in PMA-activated PMNL (Figure 2.2A; time effect, $P<$ 0.001). There was no LPS by time interaction effect $(P=1.000)$ or LPS by time by cell activation effect $(P=0.995)$ but a LPS treatment effect was detected $(P<0.001)$. Activated PMNL incubated with 25 and $50 \mu \mathrm{g} / \mathrm{mL}$ of LPS had increased luminol-derived CL as early as 10 min after PMA stimulation and peaked at values 14 and $35 \%$ higher than $0 \mu \mathrm{g} / \mathrm{mL}$ of LPS (Figure 2.2A, $P<0.05$ ). This increase in luminol-dependent CL in PMNL incubated with 25 and $50 \mu \mathrm{g} / \mathrm{mL}$ of LPS remained unceasing until 240 min after cell activation. In contrast, the luminol-derived CL of PMA-activated PMNL treated with $1 \mu \mathrm{g} / \mathrm{mL}$ of LPS was not different compared with that of PMA-activated PMNL incubated with $0 \mu \mathrm{g} / \mathrm{mL}$ LPS (Figure 2.2A; $P=0.141$ ). In non-activated PMNL, increases in luminol-derived CL of PMNL treated with 25 and $50 \mu \mathrm{g} / \mathrm{mL}$ of LPS were observed 100 and $30 \mathrm{~min}$ after the initiation of the assay, relative to PMNL incubated with $0 \mu \mathrm{g} / \mathrm{mL}$ of LPS (Figure 2.2A; treatment effect, $P<0.001$; time effect $P<0.001$ ). At the end of the assay (min 240), the luminol-derived CL of non-activated PMNL incubated with 25 and $50 \mu \mathrm{g} / \mathrm{mL}$ LPS increased by 42 and $105 \%$ compared with the fluorescence of non-activated PMNL treated with $0 \mu \mathrm{g} / \mathrm{mL}$ of LPS (Figure 2.2A; treatment effect, $P<0.001)$.

Areas under the curves were generated from luminol-derived CL plotted data points for each LPS dose and cell activation status (Figure 2.2B). Independently of LPS dose, activation of PMNL with PMA increased the luminol-derived CL (AUC) relative to 
non-stimulated PMNL, (cell activation effect, $P<0.001$ ). No differences in AUC generated from $\mathrm{CL}$ measurements over time were detected between different doses of LPS, relative to the activation cell status (Figure 2.2B; LPS effect, $P=0.099$. LPS by cell activation effect, $P=0.975)$. 
Figure 2.2. Effect of Escherichia coli lipopolysaccharide (LPS) on an index of the total reactive oxygen species (ROS) production by bovine neutrophils (PMNL).PMNL were collected from the blood of cows in midlactation $(\mathrm{n}=7)$ and incubated with $0,1,25$ or $50 \mu \mathrm{g} / \mathrm{mL}$ of LPS for $120 \mathrm{~min}$. Subsequently, the luminol-derived chemiluminescence (CL) was measured every 5 min over a $240 \mathrm{~min}$ period after addition of phorbol 12myristate,13-acetate (400 nM; PMA-activated PMNL) or HBSS (non-activated PMNL). CL values of wells without PMNL (containing HBSS and reagents only) were subtracted from fluorescence measurements of wells with PMA-activted and non-activated PMNL at every time point. (A) Luminol-derived CL in arbitrary units (AU) from non-activated and PMA-activated PMNL measured every 5 min over a 240 min period after activation of PMNL with PMA. Analysis of variance with a repeated measures design was used to detect differences between treatments (LPS effect, $P<0.001$; time effect, $P<0.001$; LPS by time interaction effect, $P=1.000$; LPS by time by cell activation effect, $P=0.995)$. (B) Areas under the curves (AUC) calculated from plotted luminol-derived CL measurements for non-activated and PMA-activated PMNL (activation effect, $P<0.001$ ). Error bars represent the standard error of the mean. 


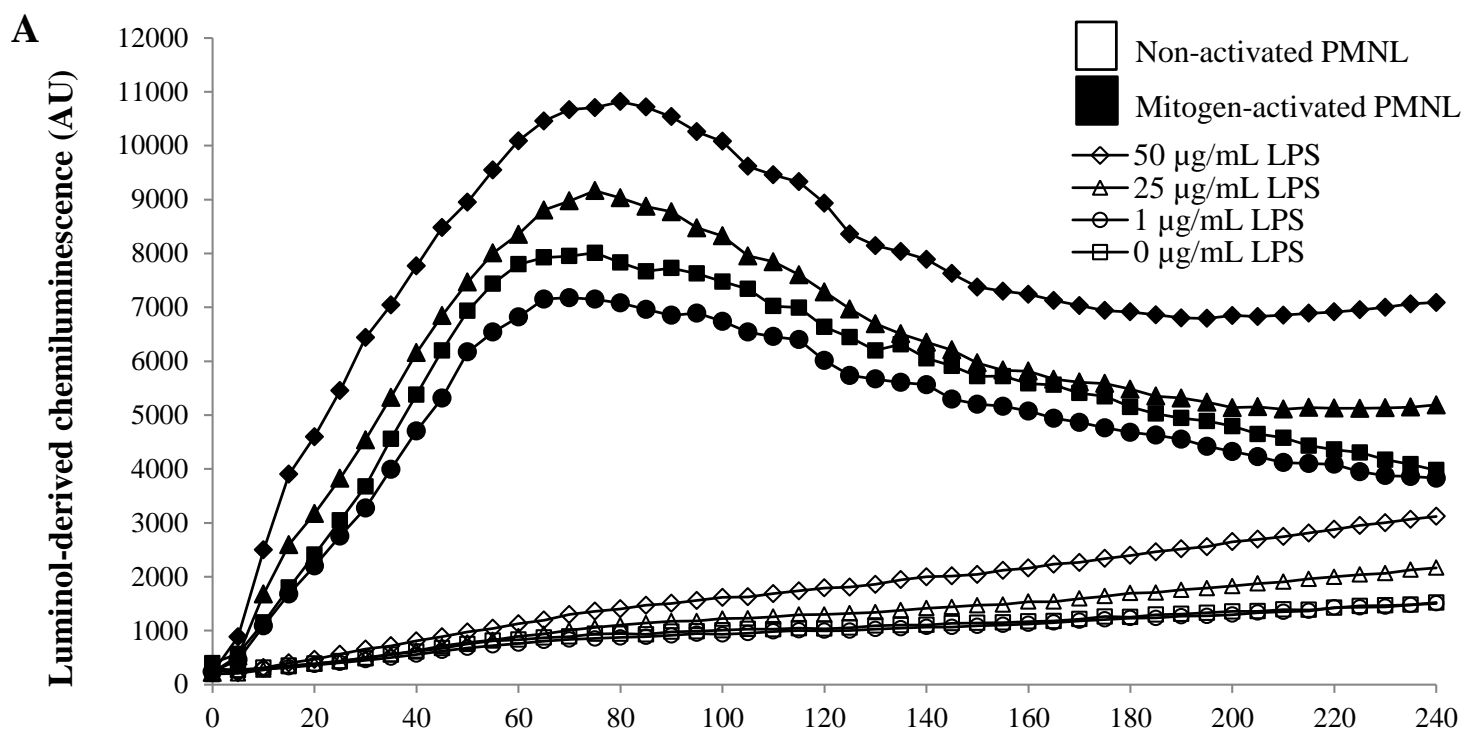

Time relative to activation,

B

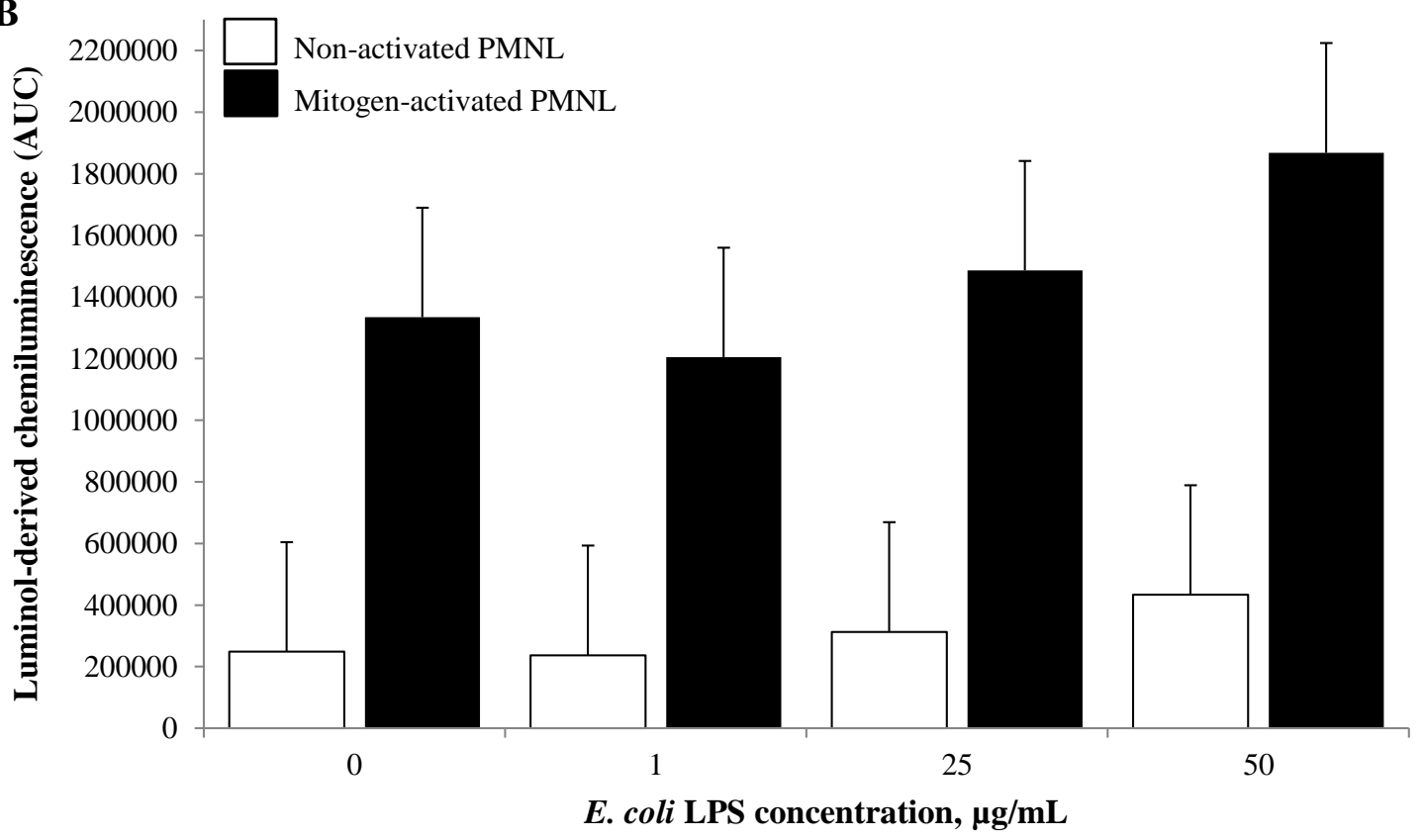




\section{Neutrophil Extracellular Trap Expression (NETs)}

Activation of PMNL with $16.21 \mathrm{nM}$ of PMA augmented NET release regardless

of LPS concentration (Figure 2.3; PMA-activation effect, $P<0.001$ ). A LPS by activation interaction effect on NET release was detected $(P=0.014)$. In non-activated PMNL, NET expression by PMNL incubated with 25 and $50 \mu \mathrm{g} / \mathrm{mL}$ of LPS was $105 \%$ higher compared with $0 \mu \mathrm{g} / \mathrm{mL}$ LPS $(P<0.05)$. However, no difference in NET expression by PMNL was detected between 1 and $0 \mu \mathrm{g} / \mathrm{mL}$ of LPS $(P=0.768)$ or 50 and $25 \mu \mathrm{g} / \mathrm{mL}$ of LPS $(P=1.000)$ in non-activated PMNL. The release of NET did not differ among any of the LPS doses when the PMNL were activated by PMA $(P>0.20)$. 


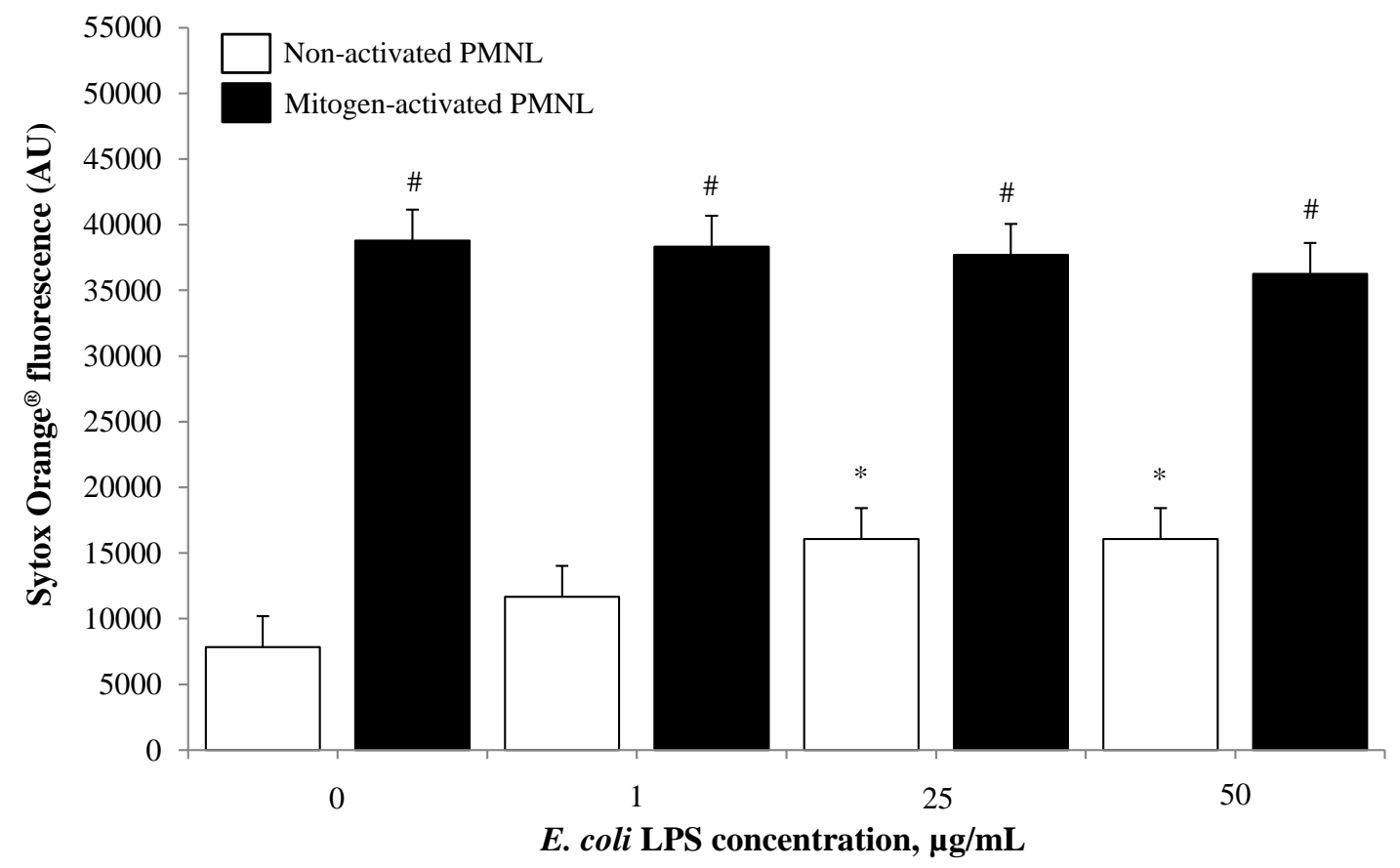

Figure 2.3. Effect of Escherichia coli lipopolysaccharide (LPS) on neutrophil extracellular traps expression (NETs).PMNL were collected from the blood of cows in midlactation ( $\mathrm{n}=7)$ and incubated with $0,1,25$ or $50 \mu \mathrm{g} / \mathrm{mL}$ of LPS for $120 \mathrm{~min}$. Bars represent mean fluorescence of cells stained with Sytox Orange (Invitrogen Co., Eugene, OR) after a 1-h incubation with 0 (non-activated PMNL) or $16.21 \mathrm{nM}$ of phorbol 12myristate,13-acetate (PMA-actiavated PMNL). Twenty five and $50 \mu \mathrm{g} / \mathrm{mL}$ of LPS increased NETs expression in non-activated PMNL (LPS by activation effect, $P=0.014$ ). Error bars represent the standard error of the mean. *Bars with an asterisk differ from 0 $\mu \mathrm{g} / \mathrm{mL}$ of LPS within their activation status at $P<0.05$ (Tukey). ${ }^{*}$ Bars with a number sign differ from non-activated PMNL within the same LPS dose at $P<0.05$ (Tukey). 


\section{Neutrophil Chemotaxis and Bactericidal Ability}

Independent of LPS dose, an average of 80 to 110 PMNL per microscopic field at $1,000 \times$ were found in membranes, after migration towards IL-8 (directed chemotaxis) while less than five PMNL/field were counted in membranes after random migration in response to CMF-HBSS (Figure 2.4; chemoattractant effect, $P<0.001$ ). Incubation with LPS had no effect on the chemotactic ability of PMNL towards IL-8 or CMF-HBSS (Figure 2.4; LPS effect, $P=0.378$; LPS by chemoattractant effect, $P=0.513$ ).

Figure 2.5 indicates the killing ability of PMNL against $S$. aureus after incubation with $0,1,25$ and $50 \mu \mathrm{g} / \mathrm{mL}$ of $E$. coli LPS. In average, PMNL killed between 35 and $45 \%$ of bacteria during the 1-hr co-incubation across all LPS concentrations. There was no effect of LPS on the bactericidal ability of PMNL against S. aureus (Figure 2.5; LPS effect, $P=0.686)$ 


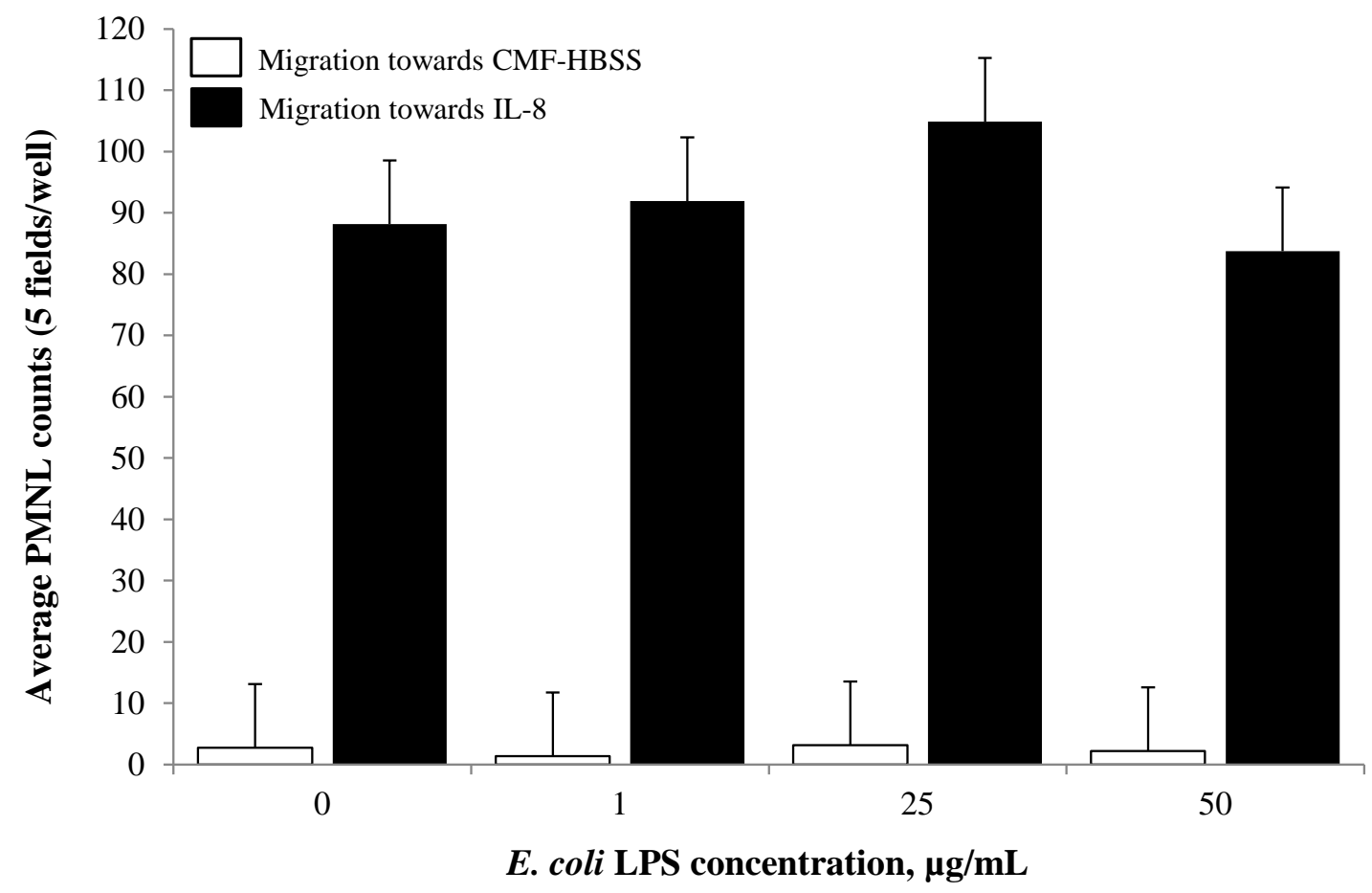

Figure 2.4. Effect of Escherichia coli lipopolysaccharide (LPS) on the chemotaxis of bovine neutrophils (PMNL) towards the chemoattractant interleukin-8 (IL-8).PMNL were collected from the blood of cows in midlactation $(n=7)$ and incubated with $0,1,25$ or $50 \mu \mathrm{g} / \mathrm{mL}$ of LPS for $120 \mathrm{~min}$. Bars represent the mean number of migrated neutrophils towards interleukin-8 (IL-8) or CMF-HBSS observed per five fields at 1000x. LPS had no effect on the chemotactic ability of PMNL towards IL-8 (LPS effect, $P=$ 0.378 ; chemoattractant effect, $P<0.001)$. Error bars represent the standard error of the mean. 


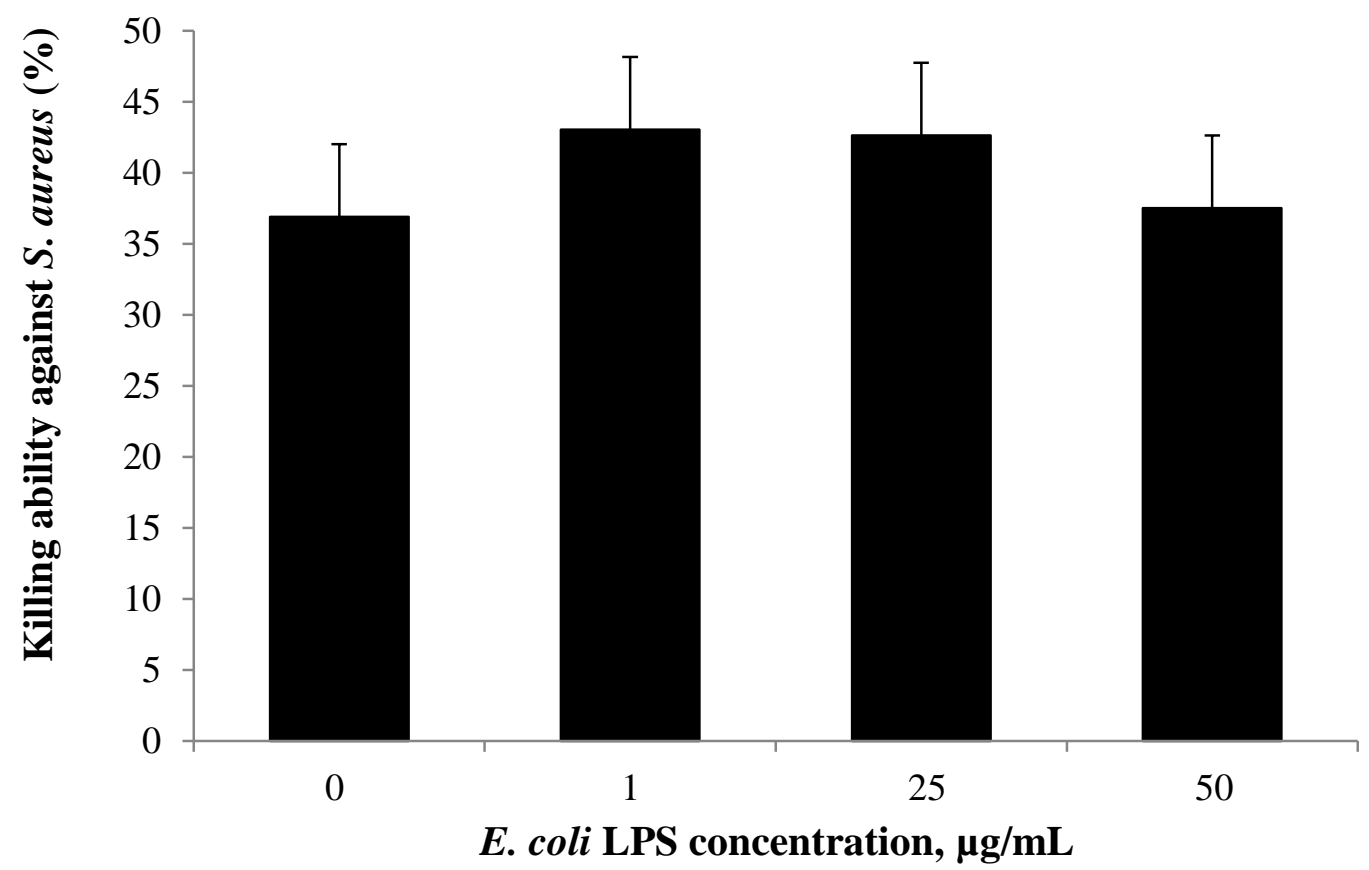

Figure 2.5. Effect of Escherichia coli lipopolysaccharide (LPS) on the bactericidal ability of neutrophils (PMNL) against Staphylococcus aureus. PMNL were collected from the blood of cows in midlactation $(\mathrm{n}=7)$ and incubated with $0,1,25$ or $50 \mu \mathrm{g} / \mathrm{mL}$ of LPS for 120 min. Bars represent the mean percentage of $S$. aureus killed in a 1-hr coculture with PMNL determined by dividing the difference in the number of bacteria incubated in the absence and presence of PMNL by the number of bacteria incubated in the absence of PMNL. LPS had no effect on the bactericidal ability of PMNL against $S$. aureus (LPS effect, $P=0.686$ ). Error bars represent the standard error of the mean. 


\section{Neutrophil Gene Expression}

There was no LPS by stage of lactation interaction effect on the PMNL expression of IL-8 mRNA (Figure 2.6A; $P=0.599$ ). However, gene expression of $I L-8$ was $537 \%$ higher in PMNL incubated with $50 \mu \mathrm{g} / \mathrm{mL}$, relative to $0 \mu \mathrm{g} / \mathrm{mL}$ LPS, and this effect was independent of stage of lactation (Figure 2.6B; LPS effect, $P<0.001$ ). Also, PMNL from midlactation tended to have $99 \%$ higher IL-8 mRNA contents compared with early lactation cows (Figure 2.6C; stage of lactation effect, $P=0.073$ ). A LPS by stage of lactation interaction effect on the expression of TNF was detected indicating a potent $2,908 \%$ increase in TNF mRNA expression in response to LPS in PMNL from midlactation but not in PMNL from early lactation cows (Figure 2.6D; LPS by stage of lactation effect, $P=0.025)$. TNF mRNA expression was $897 \%$ greater in PMNL treated with $50 \mu \mathrm{g} / \mathrm{mL}$ compared with $0 \mu \mathrm{g} / \mathrm{mL}$ LPS, regardless of stage of lactation (Figure 2.6E; LPS effect, $P<0.001)$. Expression of TNF was $482 \%$ higher in PMNL from midlactation compared with early lactation cows, independent of LPS treatment (Figure 2.6F; stage of lactation effect, $P=0.020)$.

A LPS by stage of lactation interaction effect on the PMNL expression of BPI mRNA was detected (Figure 2.7A; $P=0.012$ ). Incubation of PMNL with $50 \mu \mathrm{g} / \mathrm{mL}$ LPS decreased $(P=0.045)$ the expression of BPI mRNA by $39 \%$ in cells from early lactation cows, in contrast to PMNL from midlactation cows which BPI mRNA expression tended to increase $59 \%$ by $\operatorname{LPS}(P=0.082)$. 
Figure 2.6. Quantitative real-time PCR analysis of the expression of the inflammatory cytokines interleukin-8 (IL-8) and tumor necrosis factor (TNF) in neutrophils (PMNL). PMNL were collected from early lactation ( 7 days after calving, $\mathrm{n}=$ 10) and midlactation (146 \pm 1.7 days after calving, $\mathrm{n}=10)$ cows after incubation with 0 or $50 \mu \mathrm{g} / \mathrm{mL}$ of Escherichia coli lipopolysaccharide (LPS) for $120 \mathrm{~min}$. Bars represent the mean expression (logarithmic scale) of genes normalized by the amount of the endogenous control $\beta$-actin. (A) Effect of interaction of LPS by stage of lactation on IL- 8 mRNA expression, $P=0.599$. (B) Main effect of LPS on IL-8 mRNA expression, $P<$ 0.001. (C) Main effect of stage of lactation on IL-8 mRNA expression, $P=0.073$. (D) Effect of interaction of LPS by stage of lactation on TNF mRNA expression, $P=0.025$. (E) Main effect of LPS on TNF mRNA expression, $P<0.001$. (F) Main effect of stage of lactation on TNF mRNA expression, $P=0.020$. ${ }^{\mathrm{a}, \mathrm{b}}$ Bars with different letters differ (Tukey, $P<0.05$ ). Error bars represent the standard error of the mean. 


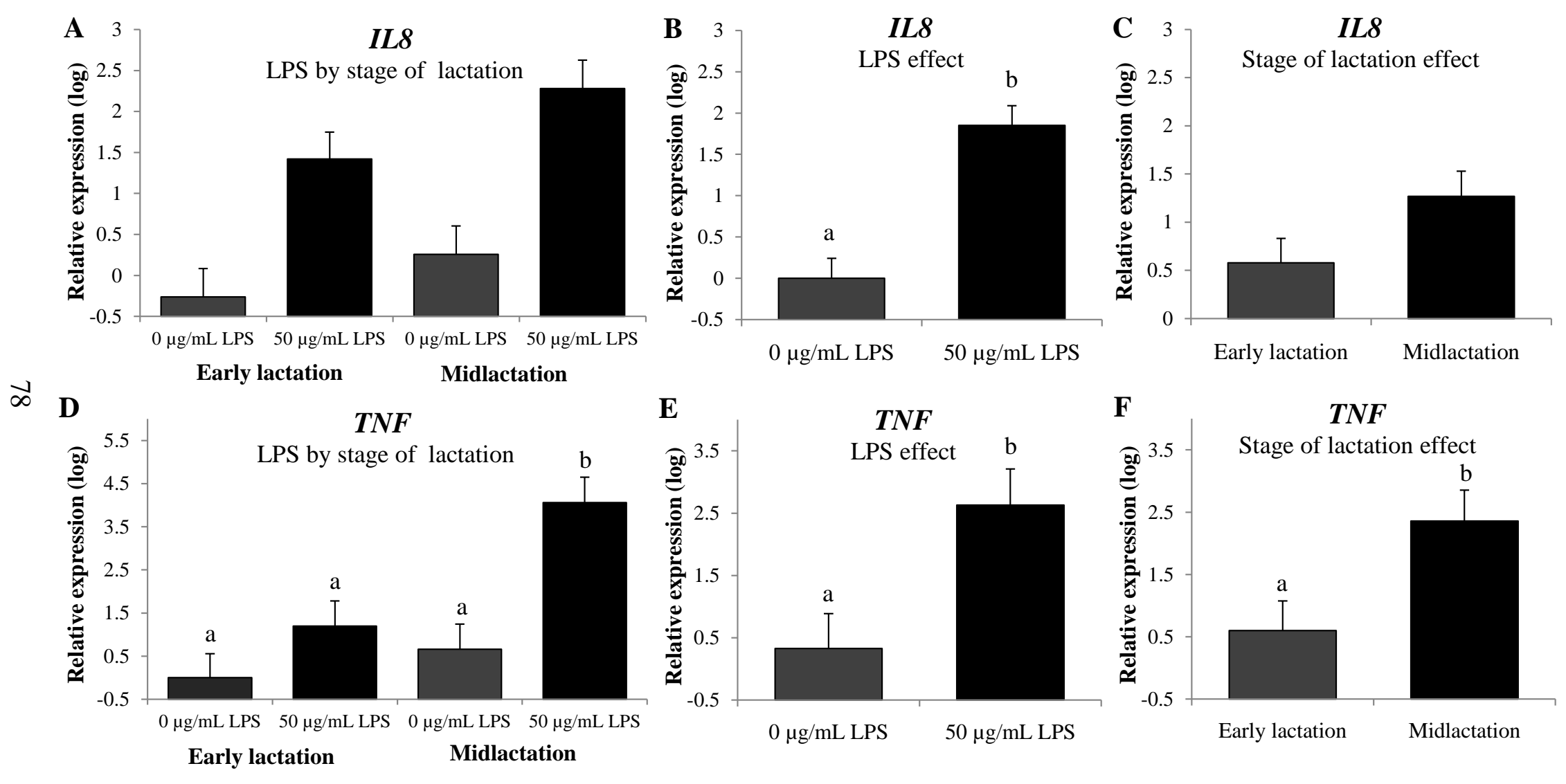



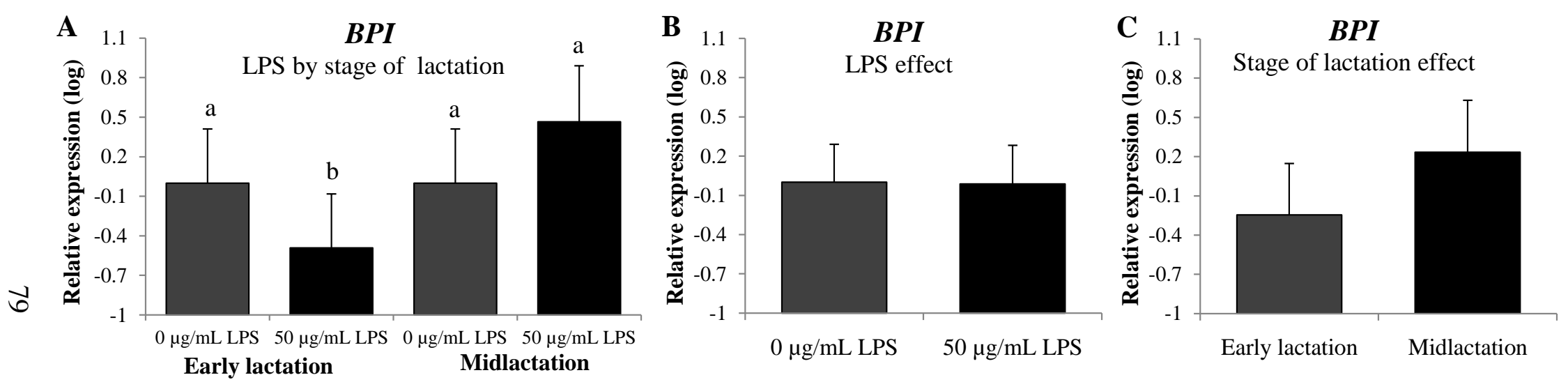

Figure 2.7. Quantitative real-time PCR analysis of bactericidal/permeability increasing protein (BPI) in neutrophils (PMNL). PMNL were collected from early lactation (7 days after calving, $\mathrm{n}=10)$ and midlactation $(146 \pm 1.7$ days after calving, $\mathrm{n}=10$ ) cows after incubation with 0 or $50 \mu \mathrm{g} / \mathrm{mL}$ of Escherichia coli lipopolysaccharide (LPS) for 120 min. Bars represent the mean expression (logarithmic scale) of genes normalized by the amount of the endogenous control $\beta$-actin. (A) Effect of interaction of LPS by stage of lactation on BPI mRNA expression, $P=0.012$. (B) Main effect of LPS on BPI mRNA expression, $P=0.939$. (C) Main effect of stage of lactation on BPI mRNA expression, $P=0.404 .{ }^{\mathrm{a}, \mathrm{b}}$ Bars with different letters differ (orthogonal contrast analysis, $P<0.05$ ). Error bars represent the standard error of the mean. 
There was no LPS by stage of lactation interaction effect on the PMNL expression of MPO mRNA (Figure 2.8A; $P=0.632$ ). Incubation with LPS did not alter the expression of MPO mRNA in PMNL, regardless of stage of lactation (Figure 2.8B; LPS effect, $P=0.188$ ). However, neutrophils from early lactation cows had $634 \%$ higher mRNA contents of MPO, compared with midlactation cows (Figure 2.8C; stage of lactation effect, $P<0.001)$. There was no LPS by stage of lactation interaction effect on the expression of PMNL SOD2 mRNA (Figure 2.8D; $P=0.942$ ). However, contents of SOD2 mRNA were $45 \%$ higher in PMNL incubated with $50 \mu \mathrm{g} / \mathrm{mL}$, compared with 0 $\mu \mathrm{g} / \mathrm{mL}$ LPS (Figure 2.8E; LPS effect, $P=0.034$ ), but were not affected by stage of lactation (Figure 2.8F; stage of lactation effect $P=0.404$ ). There was no LPS by stage of lactation interaction effect on the PMNL mRNA contents of NOX4 (Figure 2.8G, $P=$ 0.097 ) and the mRNA expression of NOX4 was not affected by LPS (Figure $2.8 \mathrm{H}, P=$ 0.596) or stage of lactation (Figure 2.8I, $P=0.846$ ). Incubation of PMNL with $50 \mu \mathrm{g} / \mathrm{mL}$ LPS decreased the expression of CYBA mRNA by $49 \%$ in cells from early lactation cows, in contrast to PMNL from midlactation cows which CYBA mRNA expression was increased $158 \%$ by LPS (Figure $2.8 \mathrm{~J}$; LPS by stage of lactation effect, $P=0.007$ ). There was no LPS by stage of lactation interaction effect on the PMNL mRNA contents of H2A/1 (Figure 2.9A, $P=0.588$ ) and H2B (Figure 2.9D; $P=0.077$ ). The gene expression of $\mathrm{H} 2 \mathrm{~A} / 1$ and $\mathrm{H} 2 \mathrm{~B}$ mRNA in blood PMNL was not affected by LPS or stage of lactation (Figures 2.9B, 2.9C, 2.9E and 2.9F, $P>0.20$ ). 
Figure 2.8. Quantitative real-time PCR analysis of the expression of the proteins involved in the production of reactive oxygen species myeloperoxidase (MPO), superoxide dismutase 2 (SOD2), NADPH oxidase 4 (NOX4), and cytochrome b-245, alpha polypeptide (CYBA) in neutrophils (PMNL). PMNL were collected from early lactation (7 days after calving, $\mathrm{n}=10$ ) and midlactation (146 \pm 1.7 days after calving, $\mathrm{n}=$ 10) cows after incubation with 0 or $50 \mu \mathrm{g} / \mathrm{mL}$ of Escherichia coli lipopolysaccharide (LPS) for $120 \mathrm{~min}$. Bars represent the mean expression (logarithmic scale) of genes normalized by the amount of the endogenous control $\beta$-actin. (A) Effect of interaction of LPS by stage of lactation on MPO mRNA expression, $P=0.632$. (B) Main effect of LPS on MPO mRNA expression, $P=0.188$. (C) Main effect of stage of lactation on MPO mRNA expression, $P<0.001$. (D) Effect of interaction of LPS by stage of lactation on SOD2 mRNA expression, $P=0.942$. (E) Main effect of LPS on SOD2 mRNA expression, $P=0.038$. (F) Main effect of stage of lactation on SOD2 mRNA expression, $P=0.404$. (G) Effect of interaction of LPS by stage of lactation on NOX4 mRNA expression, $P=0.097$. (H) Main effect of LPS on NOX4 mRNA expression, $P=0.596$. (I) Main effect of stage of lactation on NOX4 mRNA expression, $P=0.846$. (J) Effect of interaction of LPS by stage of lactation on CYBA mRNA expression, $P=0.007$. (K) Main effect of LPS on CYBA mRNA expression, $P=0.619$. (L) Main effect of stage of lactation on CYBA mRNA expression, $P=0.173$. ${ }^{\mathrm{a}, \mathrm{b}}$ Bars with different letters differ (Tukey, $P<0.05$ ). Error bars represent the standard error of the mean. 

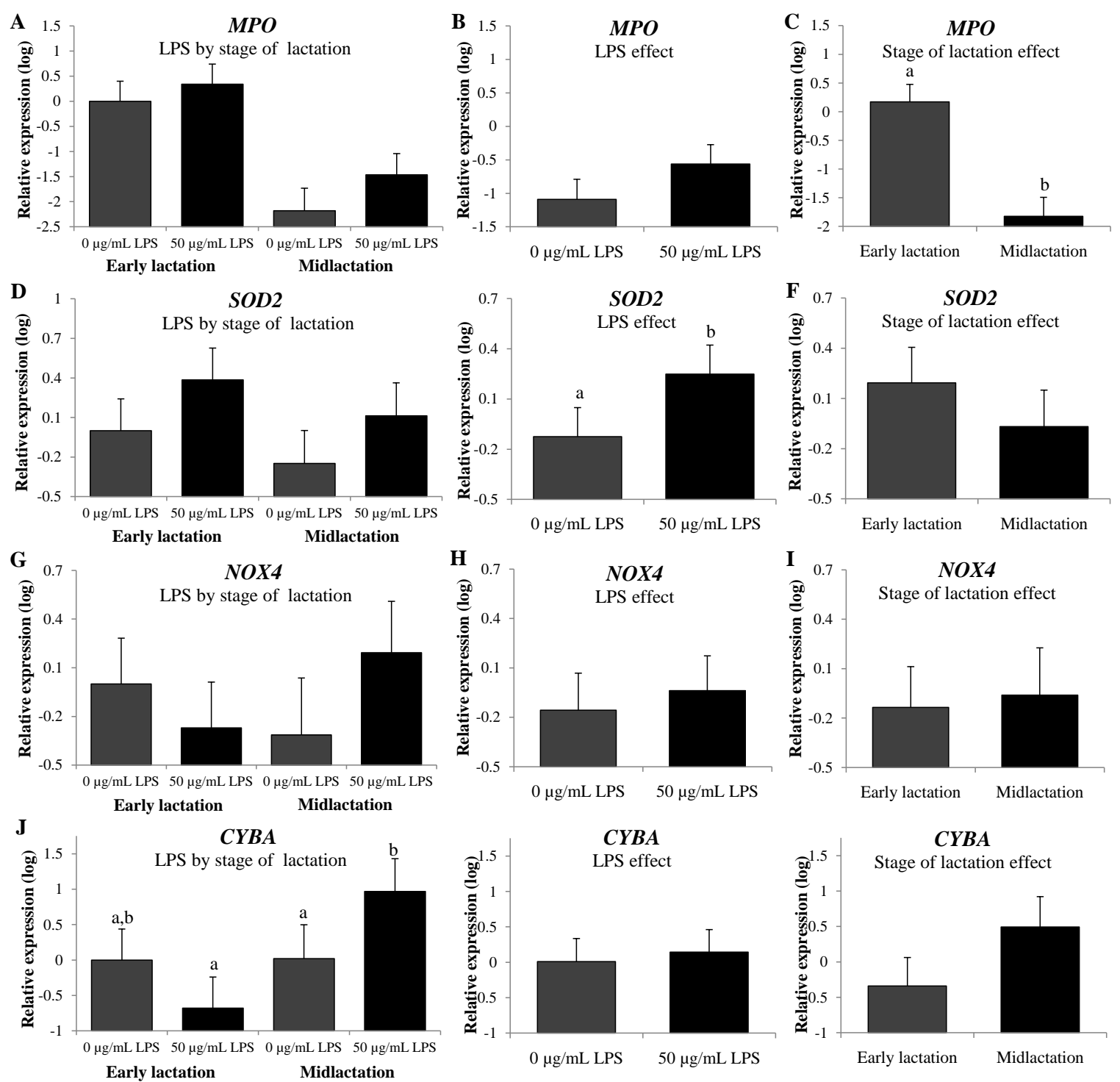
Figure 2.9. Quantitative real-time PCR analysis of the mRNA expression of the histones $H 2 A / 1$ and $H 2 B$, structural components of neutrophil (PMNL) extracellular traps. PMNL were collected from early lactation ( 7 days after calving, $n=10$ ) and midlactation (146 \pm 1.7 days after calving, $\mathrm{n}=10)$ cows and then incubated with 0 or 50 $\mu \mathrm{g} / \mathrm{mL}$ of Escherichia coli lipopolysaccharide (LPS) for $120 \mathrm{~min}$. Bars represent the mean expression (logarithmic scale) of genes normalized by the amount of the endogenous control $\beta$-actin. (A) Effect of interaction of LPS by stage of lactation on $\mathrm{H} 2 \mathrm{~A} / 1$ mRNA expression, $P=0.588$. (B) Main effect of LPS on H2A/1 mRNA expression, $P=0.939$. (C) Main effect of stage of lactation on $\mathrm{H} 2 \mathrm{~A} / 1 \mathrm{mRNA}$ expression, $P=0.289$. (D) Effect of interaction of LPS by stage of lactation on H2B mRNA expression, $P=0.077$. (E) Main effect of LPS on H2B mRNA expression, $P=0.863$. (F) Main effect of stage of lactation on H2B mRNA expression, $P=0.706$. LPS and stage of lactation had no effect on the expression of H2A/1 and H2B mRNA by bovine PMNL. Error bars represent the standard error of the mean. 

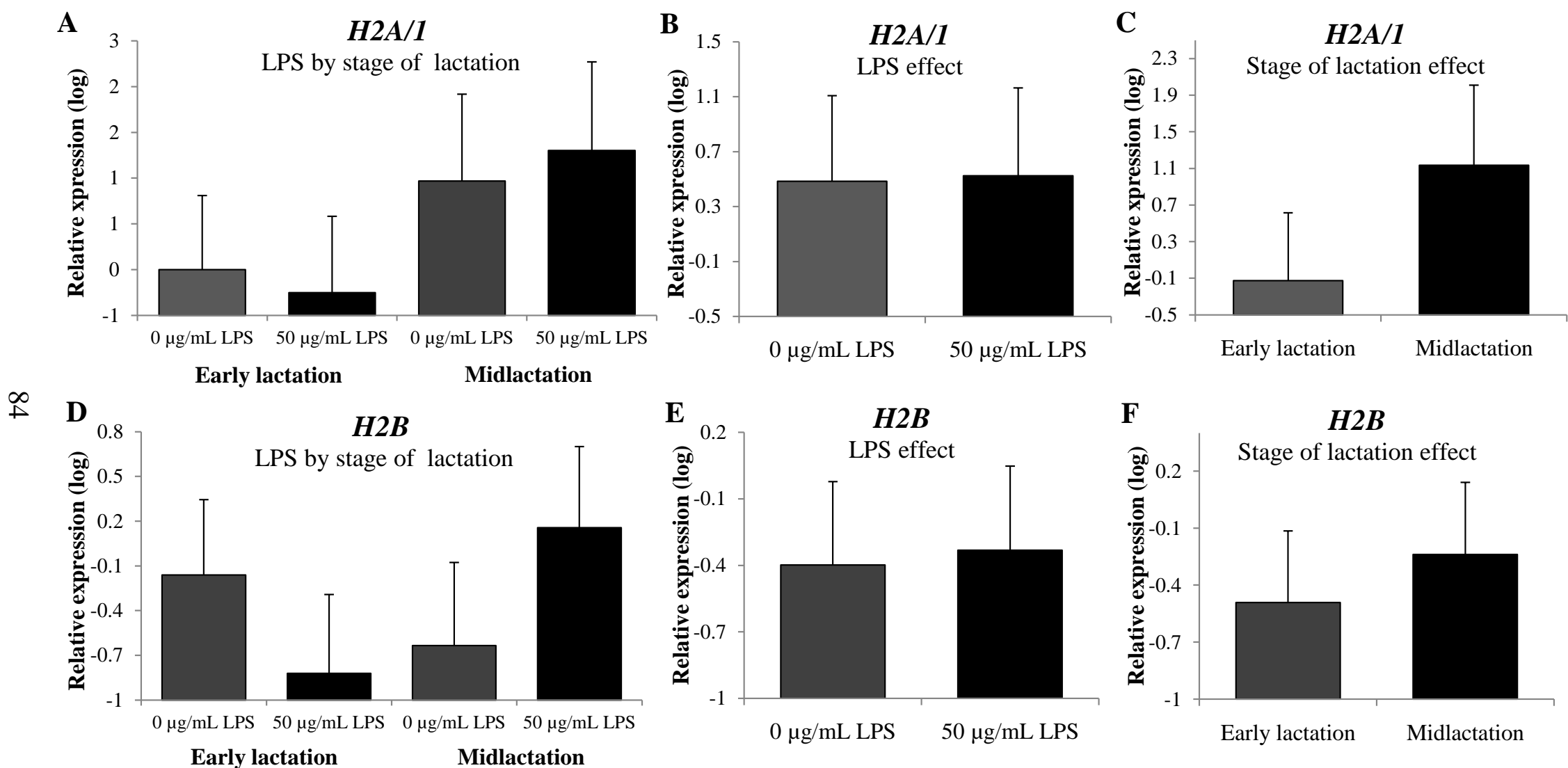


\section{DISCUSSION}

In this study, PMNL incubated with 25 and $50 \mu \mathrm{g} / \mathrm{mL}$ of $E$. coli LPS had increased oxidative burst as indicated by two independent assays that measure indices of total and intracellular ROS. The total generation of ROS was estimated by a luminoldependent assay that measures superoxide $\left(\mathrm{O}_{2}{ }^{-}\right)$, hydroxyl radical $\left(\mathrm{OH}^{-}\right)$, hydrogen peroxide $\left(\mathrm{H}_{2} \mathrm{O}_{2}\right)$, peroxynitrite $\left(\mathrm{ONOO}^{-}\right)$and hypochlorous acid $(\mathrm{HClO})$ whereas the intracellular generation of ROS was assessed using a CM- $\mathrm{H}_{2}$ DCFDA fluorescence assay that reacts only with ROS that are trapped inside the cell, including $\mathrm{H}_{2} \mathrm{O}_{2}$, nitric oxide (NO) and $\mathrm{ONOO}^{-}$(Rinaldi et al., 2007). Consistent with our findings, an increase in the production of ROS by PMNL in response to LPS in culture media has been observed in neutrophils collected from cows (McClenahan et al., 2000) and humans (Forehand et al., 1989; Aida and Pabst, 1990; Böhmer et al., 1992). McClenahan et al. (2000) incubated blood PMNL collected from healthy cows with either 0 or $10 \mu \mathrm{g} / \mathrm{mL}$ of LPS from $P$. Haemolytica for 30 min and reported an approximately $20 \%$ increase in the luminolderived luminescence after activation of PMNL with opsonized zymosan. Increased luminol-dependent ROS generation has been also observed in PMNL collected from the glands of cows after intramammary infusion with Salmonella typhimurium (Sandgren et

al., 1992) and E. coli LPS (Mehrzad et al., 2001a). Mehrzad et al. (2001a) reported that PMNL from the blood and milk of early lactation cows 12 hours after the infusion of 25 $\mu \mathrm{g} / \mathrm{mL}$ of $E$. coli LPS into one mammary quarter had increased luminol-dependent ROS production compared with that of PMNL collected from non-LPS infused quarters (Mehrzad et al., 2001a). These authors suggested that the increase in ROS production in PMNL collected from blood and LPS-infused quarters could be due to augmented plasma 
and milk concentrations of TNF- $\alpha$, IL- 1 and IL-6, respectively. However, our results and those by McClenahan et al. (2000) indicate that LPS is capable of activating bovine PMNL in a direct manner. Direct LPS activation of PMNL may occur via stimulation of the TLR-4, CD14 receptor complex (Chow et al., 1999) and is likely a calcium-dependent signal transduction process (Forehand et al., 1989).

A novel antimicrobial mechanism termed neutrophil extracellular traps (NETs) has been recently described by Brinkmann et al. (2004). Activation of PMNL with PMA, IL-8 or LPS leads to swelling of the nuclei and dissolution of its chromatin to form strands of decondensed DNA. Rupture of intracellular membranes allows mixing of these structures with granule substances including proteolytic enzymes, antimicrobial proteins, and ROS. Subsequently, these NETs are released to the extracellular matrix where they contribute to the killing ability of PMNL by delivering a high concentration of antimicrobial substances and as a barrier that prevents the microorganisms from spreading (Brinkmann et al., 2004; Brinkmann and Zychlinsky, 2007). In the current study, 25 and $50 \mu \mathrm{g} / \mathrm{mL}$ of LPS enhanced the expression of NETs by non-activated PMNL whereas LPS had no effect on NET release by PMA-activated neutrophils. It is unclear why $1 \mu \mathrm{g} / \mathrm{mL}$ of LPS had no detectable effect on NET whereas $0.1 \mu \mathrm{g} / \mathrm{mL}$ of LPS was sufficient to stimulate NET release by human PMNL incubated under similar conditions (Brinkmann et al., 2004). Previous research has shown that bovine PMNL release NETs after activation with the potent kinase C activator PMA (Lippolis et al., 2006b; Revelo and Waldron, 2010) or upon direct contact with E. coli (Grinberg et al., 2008). To our knowledge, this is the first study that demonstrates that bovine PMNL are capable of expressing NETs after direct stimulation with LPS. 
In this study, LPS had no priming effect on PMNL chemotaxis towards IL-8 as determined in vitro using a modified Boyden micro-chemotaxis chamber. Conversely, human PMNL incubated with $0.01 \mu \mathrm{g} / \mathrm{mL}$ of LPS had approximately a 76 and $55 \%$ decrease in their subsequent in vitro chemotaxis in response to the chemoattractants IL-8 and fMLP, respectively (Haslett et al., 1985; Hayashi et al., 2003). Although the physiological relevance of these findings is unclear, Haslett et al. (1985) suggested that reduced chemotaxis capacity by PMNL exposed to LPS is a consequence of increased adhesion properties of the PMNL. In dairy cows, intramammary infusion of LPS induces a massive migration of leukocytes, primarily PMNL, to the mammary gland (Saad and Ostensson, 1990; Bannerman et al., 2003). However, it is likely that this migration of PMNL occurs in response to cytokines released by LPS-stimulated macrophages and mammary epithelial cells since LPS by itself does not attract bovine blood PMNL in vitro (Persson et al., 1993).

A 120-min incubation of blood PMNL with E. coli LPS had no effect on neutrophil killing ability against $S$. aureus. In vivo, intramammary infusion of $100 \mu \mathrm{g}$ of E. coli LPS into the glands of cows experimentally infected with $S$. aureus, elevated their inflammatory response but had no effect on bacterial clearance compared with infected glands infused with PBS (Kauf et al., 2007). The reason why E. coli LPS did not increase killing of $S$. aureus in the current study and that of Kauf is unclear, but could be explained by the different mechanisms used by the PMNL to recognize and respond to Gram-positive and Gram-negative bacteria. For instance, host responses to E. coli are characterized by an acute inflammatory response whereas infections with $S$. aureus result in a more chronic state of activation (Bannerman et al., 2004). Also, cells of the innate 
immune system can discern between highly conserved motifs known as pathogenassociated molecular patterns (PAMPs). Pathogen-associated molecular patterns such as peptidoglycan (PGN) are present in most bacteria whereas LPS and lipoteichoic acid (LTA) are found only in Gram-negative and Gram-positive bacteria, respectively (Aderem and Ulevitch, 2000). Distinct TLRs are used by the innate immune system to discriminate between Gram-positive and negative bacteria. For example, TLR-4 expressed by bovine PMNL (and other cell types) recognizes LPS in conjunction with the accessory molecules LBP, CD14 and MD-2 (De Schepper et al., 2008; Worku and Morris, 2009). In contrast, TLR-2 mediates the recognition of a wide variety of products of Gram-positive bacteria such as LTA (Aderem and Ulevitch, 2000). Although TLR-2 has been found to be expressed by bovine monocytes, monocyte-derived macrophages and dendritic cells (Kwong et al., 2011), the role of TLR-2 in the recognition of Grampositive bacteria by the bovine PMNL remains to be elucidated.

Incubation of PMNL with LPS enhanced the expression of genes encoding for IL8 and TNF- $\alpha$, cytokines involved in the inflammatory response. PMNL incubated with 50 $\mu \mathrm{g} / \mathrm{mL}$ of LPS had a dramatic increase in the mRNA expression of IL-8 independent of stage of lactation, although IL-8 mRNA levels tended to be higher in PMNL collected from midlactation compared with early lactation cows (day of lactation effect, $P=0.073$ ). Our results are supported by other research indicating that E. coli LPS induces the expression of IL-8 mRNA by bovine PMNL, as determined by qualitative (Worku and Morris, 2009) and quantitative real-time PCR (Yu et al., 2010). Yu et al. (2010) incubated bovine PMNL with 0, 0.001, 0.01, 0.1 and $1 \mu \mathrm{g} / \mathrm{mL}$ LPS for 6 hours and reported that only those PMNL incubated with $1 \mu \mathrm{g} / \mathrm{mL}$ had an approximately $1,700 \%$ 
increase in the levels of IL-8 mRNA, compared to controls. The magnitude of increase in IL-8 mRNA expression after PMNL incubation with LPS was lower in our study (537\%), despite the use of a higher dose $(50 \mu \mathrm{g} / \mathrm{mL})$. Different outcomes between these studies may be explained by the shorter incubation time and the lack of serum components in the culture media used in our experiments. Incubation with LPS greatly augmented the mRNA expression of TNF in PMNL isolated from midlactation cows, but had no effect in PMNL from early lactation cows. Lipopolysaccharide has been shown to increase the mRNA levels of TNF in human (Laudanna et al., 1994) and bovine (Worku and Morris, 2009) PMNL. Although only low levels of TNF transcripts were detected, Worku and Morris (2009) reported that incubation of PMNL from midlactation cows with $0.1 \mu \mathrm{g}$ of E. coli LPS for 30 min induced the expression of TNF mRNA. Importantly, the increase in IL-8 and TNF mRNA expression observed in these studies is consistent with the secretion of these cytokines by bovine PMNL after stimulation by LPS (Sohn et al. 2007b). In our study, expression of TNF mRNA in PMNL from midlactation was approximately $482 \%$ greater than in PMNL from early lactation cows. In contrast, Sordillo et al. (1995) reported that LPS-stimulated peripheral blood mononuclear cells (PBMC) collected from periparturient cows produced more TNF than PBMC from midlactation cows. However, these authors suggested that this increase could be explained by a higher percentage of monocytes present in the mononuclear-enriched cell preparations from periparturient cows, rather than augmented production per cell. Moyes et al. (2010) collected PMNL from the blood of midlactation cows fed for either adlibitum intake or restricted to $60 \%$ of their requirements and reported that the PMNL of feed-restricted cows had a $170 \%$ fold decrease in the contents of TNF mRNA after 
intramammary infusion of Streptococcus uberis. These researchers suggested that experimentally induced negative energy balance, mimicking that of periparturient cows, may be involved in the reduced immune response observed during this period. Together, these findings suggest that PMNL have a supportive role during the innate immune response involving the production of cytokines. In turn, these cytokines may act in an endocrine, paracrine, or autocrine manner to initiate the inflammatory reaction against the invading pathogen. That the expression of IL-8 and TNF mRNA by PMNL is lower in early lactation compared to midlactation may indicate that reduced capacity of PMNL to produce these cytokines may contribute to altered immune function observed during the periparturient period (Detilleux et al., 1995).

Bactericidal/permeability-increasing protein, a glycoprotein stored in the azurophilic granules of PMNL, has several roles during the inflammatory response including LPS binding, specific bactericidal activity for Gram-negative bacteria, and activation of complement for opsonization of microorganisms prior to their phagocytosis by PMNL (Nishimura et al., 2001). In the present study, the effect of LPS by stage of lactation on BPI mRNA expression suggests that LPS decreases the expression of BPI in PMNL from early lactation cows but increases its expression in PMNL from midlactation cows, relative to non-stimulated neutrophils from cows in the same stage of lactation. In non-stimulated PMNL, protein expression of BPI in the membrane of bovine PMNL has been reported to be unchanged between periparturient and midlactation cows (Lippolis et al., 2006a). Our results suggest that PMNL may have reduced capacity to produce BPI in response to stimuli during early lactation. 
We investigated the effect of LPS and stage of lactation on genes encoding proteins that are involved in the production of ROS by PMNL such as MPO, SOD2, NOX4 and CYBA. Upon activation of PMNL, the generation of the oxidative burst is initiated by the NADPH oxidase enzyme complex that produces large amounts of antimicrobial ROS such as $\mathrm{O}_{2}{ }^{-}$and $\mathrm{H}_{2} \mathrm{O}_{2}$ (Paape et al., 2003). The enzyme system NADPH oxidase is formed by several membrane subunits including the catalytic subunit NOX4 and the cytochrome $b$-light chain subunit CYBA also known as p22-phox (Sheppard et al., 2005). Furthermore, $\mathrm{O}_{2}{ }^{-}$produced by NADPH oxidase can be converted by the manganese-containing mitochondrial protein $S O D 2$ to $\mathrm{H}_{2} \mathrm{O}_{2}$ and $\mathrm{O}_{2}$. These $\mathrm{H}_{2} \mathrm{O}_{2}$ molecules can either form potent antimicrobial agents such as free hydroxyl radicals or react with chloride ions to produce hypochlorous acid in a reaction catalyzed by $M P O$ (Holley et al., 2010). In our study, LPS increased the mRNA content of SOD2 in PMNL by $45 \%$, regardless of stage of lactation. In humans, incubation of PMNL with $0.1 \mu \mathrm{g} / \mathrm{mL}$ of LPS increased the expression of SOD2 in PMNL collected from healthy donors whereas SOD2 in patients with chronic kidney disease was diminished by LPS (Olsson et al., 2010). Another interesting finding in the current study was the decreased $C Y B A$ mRNA contents of LPS-stimulated PMNL collected from early lactation compared with those from midlactation cows. Both subunits of flavocytochrome $b$, CYBA (p22-phox) and CYBB (gp91-phox) have been cloned and sequenced in bovine PMNL (Davis et al., 1998). To our knowledge, this is the first report of a reduced expression of CYBA mRNA in LPS-stimulated PMNL from early lactation cows. In humans, a missense mutation in CYBA results in a non-functional cytochrome $b$ in patients with chronic granulomatous disease, a condition characterized by a defect in phagocyte production of $\mathrm{O}_{2}{ }^{-}$(Dinauer et 
al., 1991). Perhaps the lower expression of CYBA by bovine PMNL in early lactation contributes to the reduced capacity of PMNL to produce ROS during this period. Incubation with LPS had no effect on the mRNA expression of MPO and NOX4 by bovine PMNL. Thus, the increase in the production of ROS by PMNL after stimulation with LPS observed in this study may be mediated in part by a higher expression of SOD2 and CYBA mRNA. Furthermore, PMNL from early lactation had higher mRNA contents of MPO than PMNL from midlactation cows. Conversely, bovine PMNL MPO protein expression (Lippolis et al., 2006a) and activity (Cai et al., 1994) have been reported lower during the periparturient period, consistent with the reduction in ROS generation observed during this period. Although the reason for the higher MPO mRNA expression in early lactation observed in our study is unclear, it is possible that an increase in mRNA contents for this gene does not result in augmented protein expression or activity. Previous findings from our laboratory (Revelo and Waldron, 2010) indicate that the generation of total, intracellular and extracellular ROS by PMNL is decreased in early lactation compared with midlactation cows. As an example, some mRNAs can be retained in the nucleus of cells resulting in overestimation of their levels when compared to the expression of their corresponding protein (Gry et al., 2009).

Expression of H2A/1 and H2B mRNA was not affected by LPS treatment or stage of lactation. Lippolis et al. (2006a) reported that the protein content of $\mathrm{H} 2 \mathrm{~A} / 1$ in the cytosol of PMNL from periparturient cows was not different compared with PMNL from midlactation cows. However, the membrane of PMNL from early lactation cows had lower protein levels of $\mathrm{H} 2 \mathrm{~A} / 1$ but higher levels of H2B.f and H2B.e, compared with midlactation animals (Lippolis et al., 2006a). These authors suggested that these 
differences in histone expression between nuclear fractions are related to the ability of the PMNL to rearrange these histones at the membrane. Although the role of histones in the context of NET release is not fully understood, histones may be involved during NET killing of microbes by means of two mechanisms. First, histone post-transcriptional modifications are linked to changes of chromatin during NET formation, and second, histones have independent microbial binding and antimicrobial properties that contribute to the killing capacity of NETs (Papayannopoulos and Zychlinsky, 2009). The role of histones in NET microbial killing by bovine PMNL and their impact on the immune response of periparturient dairy cows requires further investigation.

\section{CONCLUSIONS}

Our findings suggest that E. coli LPS has a direct effect on bovine PMNL function via stimulation of their total and intracellular generation of ROS, and expression of NETs. In contrast, LPS did not alter the PMNL chemotaxis towards IL-8 or their antimicrobial capacity against $S$. aureus. PMNL incubated with $50 \mu \mathrm{g} / \mathrm{mL}$ of LPS had increased mRNA expression of the cytokines IL-8 and TNF which are involved in the inflammatory response, and the enzyme SOD2 which is involved in the formation of $\mathrm{H}_{2} \mathrm{O}_{2}$ from $\mathrm{O}_{2}^{-}$. Together, these results propose a stimulatory effect of LPS towards enhanced bovine neutrophil activity and cytokine production. It is unclear if this increased PMNL activity after exposure to LPS would be beneficial or detrimental in vivo. Regardless of LPS treatment, PMNL collected from early lactation cows had reduced contents of IL-8 but increased MPO mRNA, compared with midlactation cows. After incubation of PMNL with LPS, the mRNA expression of CYBA, a component of 
the NADPH oxidase complex was decreased in PMNL collected from early lactation compared with PMNL from midlactation cows. The lower expression of genes encoding for cytokines and enzymes involved in ROS production by PMNL isolated from the blood of early lactation cows may contribute to the altered PMNL function observed during the periparturient period. 


\title{
CHAPTER THREE
}

\section{EFFECT OF A B-VITAMIN AND FUNGAL DIETARY SUPPLEMENT ON THE FUNCTION OF NEUTROPHILS HARVESTED FROM PERIPARTURIENT DAIRY COWS}

\begin{abstract}
The objective of this study was to investigate the effect of a dietary supplement containing B-vitamins, dehydrated yeast, and Trichoderma longibrachiatum fermentation products on the functional capacity of neutrophils (PMNL) isolated from the blood of dairy cows during the periparturient period. Cows received 56 g/day of either OmniGen$\mathrm{AF}^{\circledR}(\mathrm{n}=8)$ or sham control (soybean hulls; $\mathrm{n}=12$ ) mixed into total-mixed rations from day $46 \pm 1$ before calving until day 31 after parturition. Neutrophils were collected from cows on days $49 \pm 2,28 \pm 1,19 \pm 1$, and $9 \pm 1$ prepartum and 1, 7, 14, and 30 postpartum to determine their luminol-derived generation of reactive oxygen species (ROS), formation of extracellular traps (NETs), chemotaxis towards interleukin-8 (IL-8) and antimicrobial capacity against Staphylococcus aureus. No differences were detected in the generation of ROS produced by the PMNL, NETs release, chemotaxis, or killing of $S$. aureus between PMNL collected from OmniGen- $\mathrm{AF}^{\circledR}$-supplemented and nonsupplemented cows. Independent of dietary supplementation, the amount of ROS produced by PMNL increased by $39 \%$ between days 49 and 19 prepartum, then decreased by $61 \%$ to reach lowest levels on days 1,7 , and 14 postpartum, and only recovered to the levels found prepartum by day 30 after calving. Also, the release of NETs was highest on day 49 prepartum and then declined by $40 \%$ to reach the lowest level on day 19
\end{abstract}


prepartum. No changes in the release of NETs were detected between days 28 prepartum and 14 postpartum, however, the expression of NETs slightly recovered by day 30 postpartum. Finally, PMNL chemotaxis towards IL-8 and killing ability against $S$. aureus did not change during the periparturient period, regardless of dietary supplementation. These findings suggest that dietary supplementation with OmniGen- $\mathrm{AF}^{\circledR}$ does not improve most aspects of PMNL function in periparturient dairy cows. Furthermore, impairment of ROS production and release of NETs likely contribute to the altered bovine PMNL antimicrobial capacity observed during the periparturient period. 


\section{INTRODUCTION}

Polymorphonuclear leukocytes or neutrophils (PMNL) are a primary and critical component of the innate immune response to intramammary infections (IMI). In fact, efficient migration of PMNL into the mammary gland and subsequent antimicrobial activity are crucial for the resolution of an IMI (Vangroenweghe et al., 2005). In the infected tissue, PMNL recognize, contain and eliminate the invading pathogen via intracellular killing and discharge of reactive oxygen species (ROS) and antimicrobial proteins (Paape et al., 2003). Also, in a process of cell death different from apoptosis and necrosis, PMNL extrude granular proteins and fibrils of decondensed chromatin to form extracellular structures known as neutrophil extracellular traps (NETs). These NETs bind microorganisms to prevent them from distributing throughout the tissue, while keeping them in a high local concentration of antimicrobial agents which provide their killing (Brinkmann et al., 2004; Brinkmann and Zychlinsky, 2007). The capacity of PMNL to use these strategies to eliminate the invading pathogen seems to be altered during the periparturient period in dairy cows (Kehrli et al., 1989; Cai et al., 1994; Revelo and Waldron, 2010). Incompetence of host defense mechanisms to detect and eliminate the invading pathogen may contribute to increased incidence and severity of IMI during the periparturient period (Mallard et al., 1998). Despite our increased understanding of the biology of the periparturient cow in the last few decades, the incidence and severity of infectious diseases have not declined (LeBlanc et al., 2006). For instance, the prevalence of bovine clinical mastitis in the United States augmented from 13.4 to $16.5 \%$ between 1996 and 2007 (USDA, 2008). Although the causes of immune dysfunction during the periparturient period are unclear, factors associated with the increased nutritional and 
metabolic demands imposed by pregnancy and lactation may be responsible (Goff and Horst, 1997). Insufficient dietary vitamins, trace minerals, and antioxidants has been proposed to be one of the causes of altered immune competence of periparturient dairy cows (Goff, 2006).

The dairy cow requirements for B-vitamins have been estimated by the NRC (2001) based on lactating sow needs adjusted for body weight and milk production. Limited research has been conducted to determine the amounts of B-vitamins required to support gestation, milk production and the health of dairy cows (NRC, 2001). Perhaps, with the exception of biotin, rumen microorganisms are capable of synthesizing significant amounts of B-vitamins (Schwab et al., 2006). This concept has led to dogma that ruminal synthesis and escape of dietary sources are sufficient to meet the requirements of dairy cows (NRC, 2001). However, dietary supplementation with thiamin (Shaver and Bal, 2000), biotin (Chen et al., 2011), and folic acid (Graulet et al., 2007) have increased milk production suggesting that endogenous synthesis of Bvitamins may not support optimal lactation performance. In humans, deficiencies in the vitamins $\mathrm{B}_{6}, \mathrm{~B}_{12}$, and folate impair lymphocyte growth and proliferation, natural killer cell activity, and cytokine production (reviewed by Wintergerst et al., 2007). Thus, it is likely that B-vitamin supplementation is also needed to support immune health, in particular during the periparturient period, when dairy cows are more susceptible to IMI.

The dietary supplement OmniGen-AF ${ }^{\circledR}$ (Prince Agri Products, Inc. Quincy, IL), which contains a blend of B-vitamins (vitamin B-12, riboflavin, d-pantothenic acid, thiamin, niacin, vitamin B-6, folic acid, choline, and biotin), dehydrated yeast, and dried Trichoderma longibrachiatum fermentation products, has been reported to influence the 
immune responses of several species. In addition to water-soluble vitamins, OmniGen$\mathrm{AF}^{\circledR}$ contains products of yeast and fungal organisms which may provide indirect health benefits to the animal by increasing nutrient utilization. In dairy cows, dietary supplementation with OmniGen- $\mathrm{AF}^{\circledR}$ during the dry period has been shown to influence the expression of several genes involved in inflammatory signaling, cytokine binding, and apoptosis in PMNL collected within hours after parturition (Wang et al., 2009b). Supplementation with OmniGen- $\mathrm{AF}^{\circledR}$ has also restored the protein level of L-selectin in PMNL from dexamethasone-immunosuppressed sheep (Wang et al., 2007). Furthermore, using a mouse model of infection, Rowson et al. (2011) reported that feeding OmniGen$\mathrm{AF}^{\circledR}$ for 2 weeks reduced DNA accumulation of E. coli, S. aureus and S. uberis in mammary tissue after an intrammamary challenge with these pathogens. Together, these findings suggest that OmniGen- $\mathrm{AF}^{\circledR}$ may enhance the host defense mechanisms against infections by improving the ability of PMNL to eliminate the invading pathogen. Therefore, the objective of this study was to determine the effects of the dietary supplement OmniGen- $\mathrm{AF}^{\circledR}$ on PMNL function in dairy cows during the periparturient

period. We hypothesized that PMNL from cows supplemented with OmniGen-AF ${ }^{\circledR}$ would have enhanced generation of ROS, release of NETs, chemotaxis, and antimicrobial ability, relative to non-supplemented animals.

\section{MATERIALS AND METHODS}

\section{Experimental Design, Animals, and Treatments}

The use and care of all animals were approved by The University of Missouri Animal Care and Use Committee. This experiment was conducted as a completely 
randomized design with cow as the experimental unit. All cows enrolled in the study did not experience any major clinical event during the previous lactation, had an average previous lactation somatic cell count lower than 300,000 cells/ml and a current locomotion score lower than two (Sprecher et al., 1997). Initially, 32 pregnant Holstein cows entering second or greater lactation were enrolled in this study. Twelve cows developed clinical health complications as diagnosed by staff veterinarians during the study and therefore were removed from the experiment. The remaining cows received 56 $\mathrm{g} /$ day of either OmniGen- $\mathrm{AF}^{\circledR}(\mathrm{n}=8)$ or sham control (soybean hulls; $\mathrm{n}=12$ ) mixed into total-mixed rations from day $46 \pm 1$ before calving until day 31 after parturition. OmniGen- $\mathrm{AF}^{\circledR}$ is a dietary supplement manufactured by Prince Agri Products, Inc. (Quincy, IL) that contains a mixture of B-complex vitamins, dehydrated yeast, and dried Trichoderma longibrachiatum fermentation products (Table 3.1). All cows were fed diets formulated to meet or exceed nutritional recommendations for all nutrients (Table 3.2; NRC, 2001). Cows were housed in a free-stall barn, milked twice daily and fed for ad libitum feed consumption via Calan doors. Treatment groups were balanced for parity, mature equivalent 305 milk production, and body condition score. A pre-treatment blood sample was collected on day $49 \pm 2$ prior to calving and was followed by initiation of dietary treatments. To determine the effect of the dietary supplement OmniGen-AF ${ }^{\circledR}$ on the function of circulating PMNL, blood was collected on days $28 \pm 1,19 \pm 1$, and $9 \pm 1$ prepartum and 1, 7, 14, and 30 postpartum. Approximately $60 \mathrm{~mL}$ of blood were collected via coccygeal vessel puncture into evacuated tubes containing acid-citrate dextrose (Vacutainer®, Becton Dickinson, Franklin Lakes, NJ), inverted 3 times and 
stored on ice until laboratory processing (< $20 \mathrm{~min}$ ). All functional assays were performed simultaneously on the same day for any given cow. 
Table 3.1. Composition of the dietary supplement Omnigen- $\mathrm{AF}^{\circledR}$

\begin{tabular}{lcc}
\hline Nutrients $^{1}$ & Concentration, $\mathrm{mg} / \mathrm{kg}$ & ${\text { Supplemented, } \mathrm{mg} / \mathrm{day}^{2}}^{2}$ \\
\hline Vitamin B-12 & 198.4 & 11.11 \\
Riboflavin & 14.3 & 0.80 \\
D-pantothenic acid & 152.1 & 8.52 \\
Thiamin & 75.0 & 4.20 \\
Niacin & 496.0 & 27.78 \\
Vitamin B-6 & 44.1 & 2.47 \\
Folic acid & 6.6 & 0.37 \\
Choline & 77.2 & 4.32 \\
Biotin & 2.2 & 0.12 \\
\hline
\end{tabular}

Omnigen- $\mathrm{AF}^{\circledR}$ is manufactured by Prince Agri Products, Inc. (Quincy, IL).

${ }^{1}$ Ingredients: Hydrated sodium calcium aluminosilicate, diatomaceous earth, brewers dehydrated yeast, mineral oil, calcium carbonate, dried Trichoderma longibrachiatum fermentation products, rice hulls, niacin supplement, biotin, d-calcium pantothenate, vitamin B-12 supplement, choline chloride, thiamine mononitrate, pyridoxine hydrochloride, silicon dioxide, riboflavin-5-phosphate and folic acid.

${ }^{2}$ Cows in the supplemented group received $56 \mathrm{~g} /$ day of OmniGen- $\mathrm{AF}^{\circledR}(\mathrm{n}=8)$. Animals in the control group obtained $56 \mathrm{~g} /$ day of soybean hulls $(\mathrm{n}=12)$. 
Table 3.2. Chemical composition of the prepartum and postpartum diets (DM basis)

\begin{tabular}{|c|c|c|}
\hline Nutrient & Prepartum diet ${ }^{1,2}$ & Postpartum diet ${ }^{1,2}$ \\
\hline Crude Protein, $\%$ & 12.6 & 17.4 \\
\hline Available Protein, \% & 11.5 & 16.1 \\
\hline ADICP, \% & 1.0 & 1.3 \\
\hline Acid Detergent Fiber, $\%$ & 33.5 & 21.1 \\
\hline Neutral Detergent Fiber, \% & 50.3 & 30.6 \\
\hline Crude Fat, \% & 2.2 & 4.4 \\
\hline TDN, \% & 61.7 & 71.8 \\
\hline NEL, Mcal/kg & 1.4 & 1.7 \\
\hline NEm, Mcal/kg & 1.3 & 1.7 \\
\hline $\mathrm{NEg}, \mathrm{Mcal} / \mathrm{kg}$ & 0.7 & 1.1 \\
\hline Soluble Protein, \% CP & 20.2 & 26.3 \\
\hline Lignin, \% & 4.7 & 4.2 \\
\hline NDICP, $\%$ & 4.1 & 5.2 \\
\hline Starch, \% & 13.5 & 27.4 \\
\hline NFC, $\%$ & 30.7 & 44.7 \\
\hline Calcium, \% & 0.86 & 1.00 \\
\hline Phosphorus, \% & 0.23 & 0.28 \\
\hline Magnesium, \% & 0.38 & 0.36 \\
\hline Potassium, $\%$ & 1.36 & 1.39 \\
\hline Sodium, $\%$ & 0.10 & 0.33 \\
\hline Iron, ppm & 336.0 & 335.7 \\
\hline Zinc, ppm & 77.2 & 100.2 \\
\hline Copper, ppm & 22.2 & 28.2 \\
\hline Manganese, $\mathrm{ppm}$ & 77.7 & 81.0 \\
\hline Molybdenum, ppm & 1.3 & 1.4 \\
\hline Cobalt, ppm & 0.4 & 0.5 \\
\hline Sulfur, \% & 0.22 & 0.25 \\
\hline Ash, \% & 8.4 & 8.0 \\
\hline
\end{tabular}

${ }^{\mathrm{T}}$ Diets were formulated to meet or exceed nutritional recommendations for all nutrients (NRC, 2001).

${ }^{2}$ Values represent averages of samples composited every $4 \mathrm{wk}$. 


\section{Reagents}

Percoll $^{\circledR}$ was obtained from GE Healthcare Bio-Sciences Corp. (Piscataway, NJ). Luminol (5-amino-2,3-dihydro-1,4-phthalazinedione) and phorbol 12-myristate,13acetate (PMA) were obtained from Sigma Chemical Co. (St. Louis, MO) and prepared as 89.4 $\mathrm{m} M$ and 162.12 $\mu M$ stock solutions, respectively, in dimethyl sulfoxide (DMSO). Sytox ${ }^{\circledR}$ Orange nucleic acid stain was obtained from Invitrogen Co. (Carlsbad, CA) as a 5 $\mathrm{m} M$ solution in DMSO. Recombinant-human interleukin-8 (IL-8) was obtained from Sigma Chemical Co. All stock solutions were stored at $-20^{\circ} \mathrm{C}$. All working solutions, cell suspensions and reactions were prepared using endotoxin-free, calcium and magnesiumfree Hanks' Balanced Salt Solution (CMF-HBSS; Sigma Chemical Co.) or RPMI-1640 (GIBCO, Grand Island, NY) and were pre-warmed to $37^{\circ} \mathrm{C}$ immediately prior to their use in the assays. In our in vitro cellular system, CMF-HBSS and RPMI-1640 were used to maintain a balanced environment and provide the cells with water and inorganic ions essential for normal cell metabolism. The use of HBSS as buffering medium during PMNL-chemiluminescence assays results in maximal light emission and cell activation compared to PBS and Tris (Freitas et al., 2009).

\section{Isolation of Bovine Neutrophils}

Neutrophils were isolated using procedures modified from Weber et al. (2001) as validated in our laboratory. Blood $(10 \mathrm{~mL})$ was aliquotted into $50-\mathrm{mL}$ polypropylene conical tubes (Corning Inc.) and centrifuged at $1000 \times g$ for $20 \mathrm{~min}$ at $4^{\circ} \mathrm{C}$ to separate plasma from the cell pack. Plasma was discarded and the remaining cells were suspended in $35 \mathrm{~mL}$ of CMF-HBSS. The suspension was gently pipetted down the side of a $50-\mathrm{mL}$ polypropylene conical tube containing $10 \mathrm{~mL}$ of $1.084 \mathrm{~g} / \mathrm{mL}$ of Percoll ${ }^{\circledR}$. The cells were 
then subjected to gradient centrifugation ( $400 \times \mathrm{g}$ for $40 \mathrm{~min}$ at $22^{\circ} \mathrm{C}$ ). The supernatant, mononuclear cell layer and Percoll ${ }^{\circledR}$ were aseptically aspirated and discarded. The pellet (PMNL and erythrocytes) was retained and erythrocytes were lysed by hypotonic shock. For this procedure, two volumes of an ice-cold $10.56 \mathrm{~m} M \mathrm{Na}_{2} \mathrm{HPO}_{4}, 2.67 \mathrm{mM} \mathrm{NaH} \mathrm{PO}_{4}$ solution were added to one volume of cell suspension and the solution was mixed by gentle aspiration and inversion. Tonicity was restored by the addition of one volume of a $10.56 \mathrm{~m} M \mathrm{Na}_{2} \mathrm{HPO}_{4}, 2.67 \mathrm{~m} M \mathrm{NaH}_{2} \mathrm{PO}_{4} 0.43 M \mathrm{NaCl}$ solution. The cell solution was then centrifuged $(850 \times \mathrm{g})$ for $4 \mathrm{~min}$ at $4^{\circ} \mathrm{C}$ and the pellet washed twice by resuspension in $35 \mathrm{~mL}$ of CMF-HBSS and recentrifugation $\left(850 \times \mathrm{g}\right.$ for $4 \mathrm{~min}$ at $\left.4^{\circ} \mathrm{C}\right)$. Cells were enumerated and viability was determined by propidium iodide (Sigma Chemical Co.) exclusion using a Cellometer Vision ${ }^{\circledR}$ automatic cell counter (Nexcelom Bioscience LLC., Lawrence, MA).

\section{Luminol Chemiluminescence Assay}

An index of the total neutrophil ROS production was immediately assessed by a luminol chemiluminescence assay as described by Revelo and Waldron (2010). Fifty microliters of cell suspension were pipetted into the wells $\left(2.5 \times 10^{5}\right.$ cells/well $)$ of whitewalled, clear-bottomed 96-well microplates (Corning Inc.). All reactions were carried out in sixtuplicates. Twenty $\mu \mathrm{L}$ of 5mM 5-amino-2,3-dihydro-1,4-phthalazinedione (luminol) and $20 \mu \mathrm{L}$ of either $400 \mathrm{n} M$ PMA (stimulated cells) or HBSS (non-stimulated cells) were added to all wells. Reactions were then adjusted to $200 \mu \mathrm{L}$ using CMF-HBSS. Chemiluminescence (CL) was measured every $5 \mathrm{~min}$ for $240 \mathrm{~min}$ with a Synergy HT plate reader (BioTek Instruments, Inc., Winooski, VT). Background values, defined as the mean CL values of luminol diluted in CMF-HBSS, were automatically subtracted 
from all readings. Individual curves were generated from plotted CL values over time for each experimental condition. The area under the curve (AUC), maximal CL, and time to maximal CL were obtained from plotted CL values.

\section{Neutrophil Extracellular Trap (NET) Assay}

Neutrophil extracellular trap formation was quantified using Sytox ${ }^{\circledR}$ Orange as described by Revelo and Waldron (2010). After PMNL incubation with LPS, $100 \mu \mathrm{L}$ of cell suspension were pipetted into the wells $\left(6.25 \times 10^{5}\right.$ cells/well) of white-walled, clearbottomed 96-well microplates (Costar Inc., NY). All reactions were performed in sixtuplicates. Twenty microliters of PMA and $20 \mu \mathrm{L}$ of ionomycin to obtain a final concentration of $10 \mathrm{ng} / \mathrm{ml}$ and $1 \mu M$, respectively, were added to the stimulated set of wells. Forty microliters of RPMI- $1640+2 \%$ heat-inactivated FBS were added to the control wells. The plates were then centrifuged $(10 \mathrm{~min}$ at $650 \times g)$ and incubated $\left(37^{\circ} \mathrm{C}\right.$ and 5\% $\mathrm{CO}_{2}$ ) for $60 \mathrm{~min}$. After incubation, supernatant from the plates was removed, and the wells were stained and protected from light with $50 \mu \mathrm{L}$ of $5 \mu M$ Sytox Orange in CMF-HBSS (15 min at room temperature). The stain was poured off, and the wells washed three times with $100 \mu \mathrm{L}$ of CMF-HBSS. A Synergy HT plate reader was used to detect fluorescence with an excitation wavelength of $530 \mathrm{~nm}$ and emission wavelength of $580 \mathrm{~nm}$. Background values, defined as the mean fluorescence values of PMA and ionomycin diluted in RPMI-1640 were subtracted from all readings.

\section{Chemotaxis Assay}

Chemotaxis was performed in a 48-well micro chemotaxis chamber (Neuro Probe Inc., Gaithersburg, MD) according to the manufacturer's recommendations. The wells in the bottom plate of the chamber were filled with $26 \mu \mathrm{L}$ of the chemoattractant interleukin 
8 (IL-8; $100 \mathrm{ng} / \mathrm{mL}$, Sigma Chemical Co.) or CMF-HBSS, previously warmed to $37^{\circ} \mathrm{C}$. A slight positive meniscus was formed when the wells were filled with the chemoattractant. A 25 x $80 \mathrm{~mm}$ polycarbonate filter (non-polyvinylpyrrolidone treated, Neuro Probe Inc.) was lowered onto the formed meniscus, allowing the middle portion of the filter to make contact first. The polycarbonate filter had pores with a diameter of $5 \mu \mathrm{m}$ to allow migration and adhesion of PMNL to the underside of the filter where they could be stained and counted. A silicone gasket was then applied on top of the filter and the top component of the micro chemotaxis chamber fastened to the bottom chamber. The chamber was pre-warmed for $10 \mathrm{~min}$ at $37^{\circ} \mathrm{C}$ before adding the PMNL to the upper chamber. Fifty-five $\mu \mathrm{L}$ of cell suspensions (3.75 x $10^{6}$ neutrophils/ml) previously incubated with 0 or $50 \mu \mathrm{g} / \mathrm{mL}$ of $E$. coli LPS were added to the top chamber and incubated at $37^{\circ} \mathrm{C}$ in humidified air with $5 \% \mathrm{CO}_{2}$ for 30 min to allow the PMNL to migrate towards the chemoattractant. After the incubation, the fluid from the top chamber was aspirated and the chamber disassembled. The side of the filter membrane that had faced the upper chamber was rinsed with CMF-HBSS and wiped off three times with a wiper blade to remove non-migrated PMNL. Migrated PMNL were adhered to the side of the filter that had faced the IL-8 (chemoattractant) or CMF-HBSS (control). The filter was immersed in methanol for 10 min, air-dried and stained in a 3.75\% Giemsa staining solution (Sigma Chemical Co.) for $1 \mathrm{hr}$. Subsequently, the membrane was rinsed in Giemsa buffer and placed with the cell-side up on a 50 × $75 \mathrm{~mm}$ microscope slide for drying. The number of PMNL that migrated through the filter was counted under oil immersion on a light microscope by counting the number of neutrophils present in 7 microscope fields at 1,000 x. Each sample was run in triplicate. The average number of 
PMNL that migrated towards CMF-HBSS (random migration) was subtracted from the average number of PMNL that migrated towards IL-8 (directed migration). Results are reported as the average number of migrated neutrophils towards IL-8 observed per seven fields.

\section{Killing Assay}

The killing assay was performed as described by Revelo and Waldron (2010). S. aureus Newbould 305 (American Type Culture Collection, Manassas, VA) was inoculated on a blood agar plate (Northeast Laboratory Services Inc., ME) and incubated overnight at $37^{\circ} \mathrm{C}$. Ten colonies were transferred from the plate to a sterile tube containing $10 \mathrm{~mL}$ of brain-heart infusion broth (Sigma Chemical Co.) and incubated overnight at $37^{\circ} \mathrm{C}$ at $225 \mathrm{rpm}$ in an orbital shaker. After the overnight incubation, the tube was sonicated to disassociate individual bacterial cells using a sonic dismembrator (Sonifier $^{\circledR}$, Branson Ultrasonics Corp., Danbury, CT) and maintained at $4^{\circ} \mathrm{C}$ to stop bacterial growth. One hundred microliters from this culture were serially diluted in CMFHBSS and $100 \mu \mathrm{L}$ of the resulting dilutions were spread on blood agar plates. The stock culture was maintained at $4{ }^{\circ} \mathrm{C}$ and the plates were incubated overnight at $37^{\circ} \mathrm{C}$ to determine the concentration [colony forming units $(\mathrm{cfu}) / \mathrm{mL}$ ] of the stock culture based on the colony counts of the spread plates. Immediately before the initiation of the killing assay, the stock culture was placed in ice and diluted in HBSS to yield a final concentration of $1.6 \times 10^{8} \mathrm{cfu} / \mathrm{mL}$. To assess PMNL killing, $200 \mu \mathrm{L}$ of S. aureus $(3.2 \times$ $\left.10^{7} \mathrm{cfu}\right)$ and $400 \mu \mathrm{L}$ of pooled, heat-inactivated bovine serum were added to sterile tubes with or without $2 \times 10^{6}$ PMNL previously incubated with the different doses of LPS (1.4 $\mathrm{mL}$ ). The ratio of bacteria to PMNL was 16:1. All reactions were performed in triplicate. 
The samples were placed on a Labquake ${ }^{\circledR}$ tube rotator for $60 \mathrm{~min}$ at $37^{\circ} \mathrm{C}$. These reactions were then placed in ice and sonicated with a sonic dismembrator for $15 \mathrm{~s}$ at amplitude of $20 \%$. Rupture of the PMNL was verified by microscopic examination. Sonication does not affect bacterial viability (Rinaldi et al., 2007; Revelo and Waldron, unpublished observation). A $100 \mu \mathrm{L}$ aliquot from each sonicated reaction was serially diluted in CMF-HBSS and $100 \mu \mathrm{L}$ quantities of the resulting dilutions was spread on blood agar plates. The plates were incubated overnight at $37^{\circ} \mathrm{C}$ and the colonies enumerated. The percentage of bacteria killed were determined by calculating the difference in the number of bacteria incubated in the absence and presence of PMNL and dividing this difference by the number of colonies when bacteria were incubated in the absence of PMNL.

\section{Statistical Analyses}

The experiment was conducted as a completely randomized design with cow as the experimental unit. Data were analyzed by analysis of variance (ANOVA) as a mixed model with repeated measures using the mixed procedure of SAS (2001). For the luminol-based assay involving multiple measurements of CL over time, the areas under the curves (AUC) were calculated from plotted data points for each experimental condition using the expand procedure of SAS with cubic spline interpolation and the trapezoid rule (SAS, 2001). For all dependent variables, extreme observations were detected using PROC REG of SAS (2001) and removed from the data set if they presented at least three of the following characteristics: standardized residual (ri) $>2$ standard deviations, high leverage (hii $\geq 2 \mathrm{pn}-1$, where $\mathrm{n}=$ number of observations and $\mathrm{p}$ $=$ number of parameters $),$ possible undue influence $[$ DFITTSi $\geq 2(\sqrt{ }$ pn-1)-1, DFBETASi 
$\geq 2(\sqrt{ } \mathrm{n})-1]$, cook's distance $(\mathrm{Di})>1$ and ratio of generalized variances (COVRATIOi) $<$ $1-3 p n-1$ or $>1+3 p n-1$ (Kaps and Lamberson, 2004). All variables followed a normal distribution as they passed the goodness-of-fit tests for normality $(P>0.05)$ implemented using the Capability procedure of SAS (2001). Data were analyzed by analysis of variance (ANOVA) as a mixed model with repeated measures using the mixed procedure of SAS (2001). The independent variables in the model statement included dietary supplementation, day relative to calving and their interaction. The random statement defined cow as a random variable. The repeated statement was used to specify a repeated measurement model with day as the repeated effect, cow as the subject and a compound symmetry covariance structure (Littell et al., 1998). Compound symmetry covariance structures were the best fit to the model as indicated by Akaike information criteria (AIC) and Swarz Bayesian information criteria (BIC) values generated by SAS. Analysis of covariance (ANCOVA) was conducted to assess the potential influence of the pretreatment measurements collected on day 46 prepartum on the outcome variables. Analysis of covariance was implemented by defining the pre-treatment value as a continuous variable in the MODEL statement but not in the CLASS statement. Pretreatment measurements were not included in the dataset during ANCOVA. For all dependent variables, the effect of covariate was non-significant $(P>0.05)$. Therefore, covariates were removed from the MODEL statement. Separate ANOVA were conducted to determine the effect of the day relative to calving on the dependent variables. These analyses included the pre-treatment measurements in the dataset. Least squares means and standard errors were generated using the LSMeans statement in conjunction with the pdiff option of SAS. Tukey's multiple comparison test was used only to assess specific 
treatment differences for those variables shown to have significant overall treatment effects in the analysis of variance. Reported means are the adjusted least squares means \pm SEM. Significance was declared when $P<0.05$.

\section{RESULTS}

\section{Reactive Oxygen Species Production}

Luminol-derived CL, an index of the overall generation of ROS by PMNL, was measured every 5 min for a period of 240 min after stimulation of PMNL with PMA (data not shown). These CL values were plotted over time and used to calculate the AUC representing the overall amount of ROS produced by the stimulated PMNL (Figures 3.1A, 3.1B, and 3.1C), the maximal luminol-derived ROS production (Figures 3.1D, 3.1E, and 3.1F), and the time required for the PMNL to reach maximal ROS generation (Figures 3.1G, 3.1H, and 3.1I). There was no effect of interaction of OmniGen- $\mathrm{AF}^{\circledR}$ supplementation by time on the total amount of ROS produced (Figure 3.1A, $P=0.722$ ), maximal ROS generation (Figure 3.1D, $P=0.952$ ), or time to maximal ROS production (Figure $3.1 \mathrm{G}, P=0.945$ ). Also, there was no main effect of dietary supplementation on the total amount of ROS produced (Figure 3.1B, $P=0.415$ ), maximal ROS generation (Figure 3.1E, $P=0.361$ ), or time to maximal ROS generation (Figure 3.1H, $P=0.094$ ). Independent of dietary supplementation, the amount of ROS produced by PMNL (AUC) increased by $39 \%$ between days 49 and 19 prepartum $(P=0.025)$, then decreased by $61 \%$ to reach the lowest level on the day after parturition $(P<0.001)$, remained unchanged on days 7 and 14 postpartum $(P>0.200)$, and started to recover by day 30 postpartum (Figure 3.1C; main effect of time, $P<0.001$ ). The maximal production of ROS did not 
differ between days 49,28, 19 and 9 prepartum $(P>0.200)$, declined by $42 \%$ between days 9 prepartum and 1 postpartum $(P=0.009)$, remained low on days 7 and 14 postpartum $(P>0.200)$, but improved by day 30 postpartum (Figure $3.1 \mathrm{~F}$, main effect of time, $P<0.001)$. Finally, the time required for the PMNL to reach maximal ROS production increased by 106 min between days 49 and 28 prepartum $(P<0.001)$ but did not change $(P>0.200)$ during the rest of the periparturient period (Figure 3.1I; main effect of time, $P<0.001)$. 
Figure 3.1. Effect of a dietary supplement containing B-complex vitamins and yeast on the reactive oxygen species (ROS) production by bovine neutrophils (PMNL). Cows received $56 \mathrm{~g} /$ day of either OmniGen- $\mathrm{AF}^{\circledR}(\mathrm{n}=8)$ or sham control (soybean hulls; $\mathrm{n}=12$ ) mixed into total-mixed rations from day $46 \pm 1$ before calving until day 31 after parturition. PMNL were collected from the blood of cows on days $49 \pm 2$ (pre-treatment sample), $28 \pm 1,19 \pm 1$, and $9 \pm 1$ prepartum and 1, 7, 14, and 30 postpartum. Subsequently, the luminol-derived chemiluminescence (CL) was measured every 5 min over a 240 min period after addition of phorbol 12-myristate,13-acetate (PMA; $400 \mathrm{nM}$ ). CL values of wells without PMNL (containing HBSS and reagents only) were subtracted from CL measurements at every time point. Areas under the curves (AUC; panels A, B, and $\mathrm{C}$ ), maximal luminol-dependent ROS production (arbitrary units; panels D, E, and F), and time to maximal ROS production (panels $\mathrm{G}, \mathrm{H}$, and I) were calculated from plotted luminol-derived CL measurements. (A) Effect of interaction of OmniGen-AF ${ }^{\circledR}$ supplementation by time on the production of ROS (AUC) by PMA-stimulated PMNL, $P$ $=0.722$. $(\mathrm{B})$ Main effect of OmniGen- $\mathrm{AF}^{\circledR}$ supplementation on the production of ROS (AUC) by PMA-stimulated PMNL, $P=0.415$. (C) Main effect of time on the production of ROS (AUC) by PMA-stimulated PMNL, P < 0.001. (D) Effect of interaction of OmniGen- $\mathrm{AF}^{\circledR}$ supplementation by time on the maximal production of ROS (arbitrary units) by PMA-stimulated PMNL, $P=0.952$. (E) Main effect of OmniGen-AF ${ }^{\circledR}$ supplementation on the maximal production of ROS (arbitrary units) by PMA-stimulated PMNL, $P=0.361$. (F) Main effect of time on maximal production of ROS (arbitrary units) by PMA-stimulated PMNL, $P<0.001$. (G) Effect of interaction of OmniGen-AF ${ }^{\circledR}$ supplementation by time on the time to maximal production of ROS by PMA-stimulated PMNL, $P=0.945$. (H) Main effect of OmniGen-AF ${ }^{\circledR}$ supplementation on the time to maximal production of ROS by PMA-stimulated PMNL, $P=0.094$. (I) Main effect of time on the time to maximal production of ROS by PMA-stimulated PMNL, $P<0.001$. Data represent adjusted least square means. Error bars indicate the standard error of the mean. ${ }^{\text {a,b }}$ Means with different letters differ at $P<0.05$ (Tukey). 

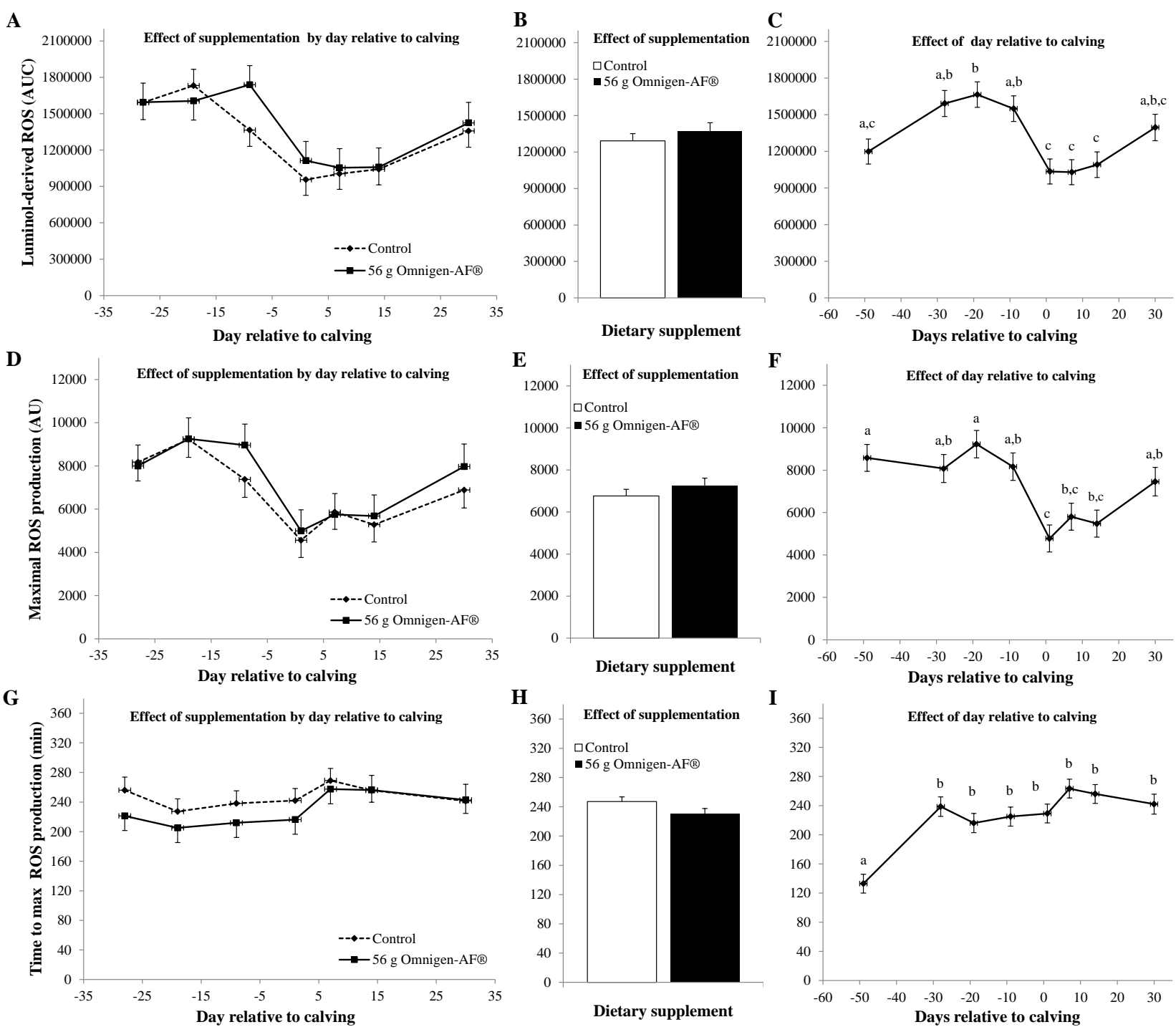


\section{Neutrophil Extracellular Trap Formation}

The release of NETs by stimulated PMNL was quantified after a 60-min incubation with PMA (Figures 3.2A, 3.2B, and 3.2C). There was no effect of interaction of OmniGen-AF ${ }^{\circledR}$ supplementation by time on the release of NETs (Figure 3.2A; $P=$ 0.332). In addition, no main effect of dietary supplementation on NETs formation was detected (Figure 3.2B; $P=0.602$ ). Regardless of dietary treatment, the release of NETs was highest on day 49 prepartum but declined by $40 \%$ to reach the lowest level on day 19 prepartum $(P<0.001)$. Changes in the formation of NETs by bovine PMNL between days 28 prepartum and 14 postpartum were not detected $(P>0.200)$, but the expression of NETs on day 30 postpartum tended $(P=0.063)$ to be $25 \%$ higher, compared with PMNL collected on day 19 prepartum (Figure 3.2C; main effect of time, $P<0.001$ ).

\section{Chemotaxis}

Directed chemotaxis towards the chemoattractant IL-8 was assessed in a microchemotaxis chamber which allows PMNL to cross the 5- $\mu \mathrm{m}$ pores of a membrane in response to IL-8. There was no effect of interaction of OmniGen-AF ${ }^{\circledR}$ supplementation by time on the chemotaxis of PMNL towards IL-8 (Figure 3.3A; $P=0.431$ ). Similarly, no main effect of dietary supplementation on the chemotaxis of PMNL towards IL-8 was detected (Figure 3.3B; $P=0.367$ ). There was no main effect of time on the chemotaxis of PMNL towards IL-8 (Figure 3.3C; $P=0.694$ ). 

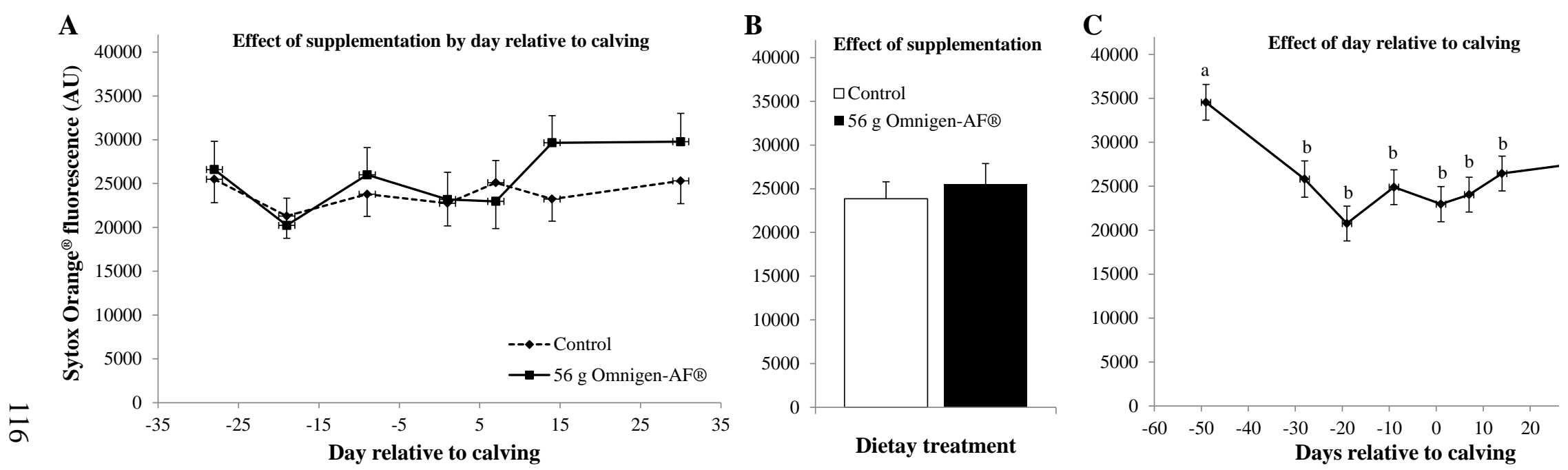

Figure 3.2. Effect of a dietary supplement containing B-complex vitamins and yeast on Neutrophil Extracellular Trap release (NET) by bovine neutrophils (PMNL). Cows received $56 \mathrm{~g} /$ day of either OmniGen-AF ${ }^{\circledR}(\mathrm{n}=8)$ or sham control $(\mathrm{soybean}$ hulls; $\mathrm{n}=12$ ) mixed into total-mixed rations from day $46 \pm 1$ before calving until day 31 after parturition. PMNL were collected from the blood of cows on days $49 \pm 2$ (pre-treatment sample), $28 \pm 1,19 \pm 1$, and $9 \pm 1$ prepartum and 1, 7, 14, and 30 postpartum. Release of NET was assessed by measuring the fluorescence of cells stained with Sytox Orange ${ }^{\circledR}$ (Invitrogen Co., Eugene, OR) after a 1-h incubation with $16.21 \mathrm{nM}$ of phorbol 12-myristate,13-acetate (PMA). (A) Effect of interaction of OmniGen- $\mathrm{AF}^{\circledR}$ supplementation by time on NET release by PMA-stimulated PMNL, $P=0.332$. (B) Main effect of OmniGen$\mathrm{AF}^{\circledR}$ supplementation on NET release by PMA-stimulated PMNL, $P=0.602$. (C) Main effect of time on NET release by PMAstimulated PMNL, $P<0.001$. Data represent adjusted least square means. Error bars indicate the standard error of the mean. ${ }^{\text {a,b }}$ Means with different letters differ at $P<0.05$ (Tukey). 

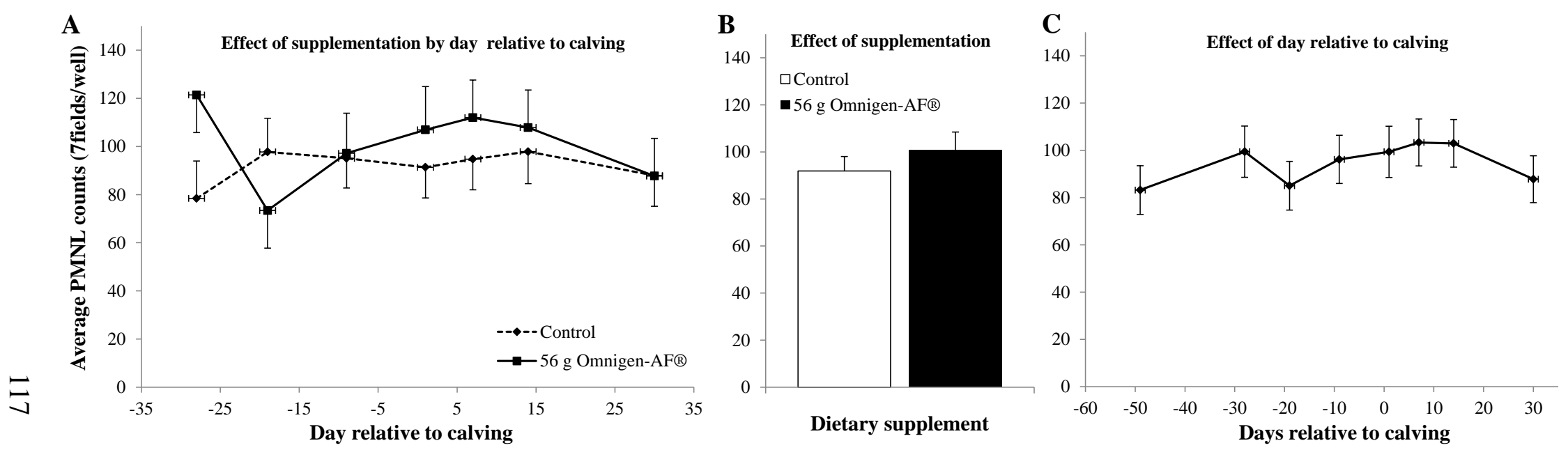

Figure 3.3. Effect of a dietary supplement containing B-complex vitamins and yeast on neutrophil (PMNL) chemotaxis towards the chemoattractant interleukin-8 (IL-8). Cows received $56 \mathrm{~g} /$ day of either OmniGen-AF ${ }^{\circledR}(\mathrm{n}=8)$ or sham control (soybean hulls; $\mathrm{n}=12$ ) mixed into total-mixed rations from day $46 \pm 1$ before calving until day 31 after parturition. PMNL were collected from the blood of cows on days $49 \pm 2$ (pre-treatment sample), $28 \pm 1,19 \pm 1$, and $9 \pm 1$ prepartum and 1, 7, 14, and 30 postpartum. Chemotaxis was determined as the mean number of migrated PMNL towards IL- 8 observed in seven fields at $1000 \mathrm{x}$. (A) Effect of interaction of OmniGen-AF ${ }^{\circledR}$ supplementation by time on chemotaxis of PMNL towards IL-8, $P=0.431$. (B) Main effect of OmniGen-AF ${ }^{\circledR}$ supplementation on chemotaxis of PMNL towards IL-8, $P=0.367$. (C) Main effect of time on chemotaxis of PMNL towards IL-8, $P=0.694$. Data represent adjusted least square means. Error bars indicate the standard error of the mean. ${ }^{\text {a,b }}$ Means with different letters differ at $P<0.05$ (Tukey). 


\section{Killing of Staphylococcus Aureus}

On average, PMNL killed approximately $37 \%$ of $S$. aureus during a 1 -hr coincubation with the pathogen. There was no effect of interaction of OmniGen-AF ${ }^{\circledR}$ supplementation by time on the killing ability of PMNL against $S$. aureus (Figure 3.4A; $P$ $=0.096)$. Also, no main effect of dietary supplementation on the killing of S. aureus by PMNL was detected (Figure 3.4B; $P=0.522$ ) and no main effect of time on the killing of S. aureus by PMNL (Figure $3.4 \mathrm{C} ; P=0.148$ ). 

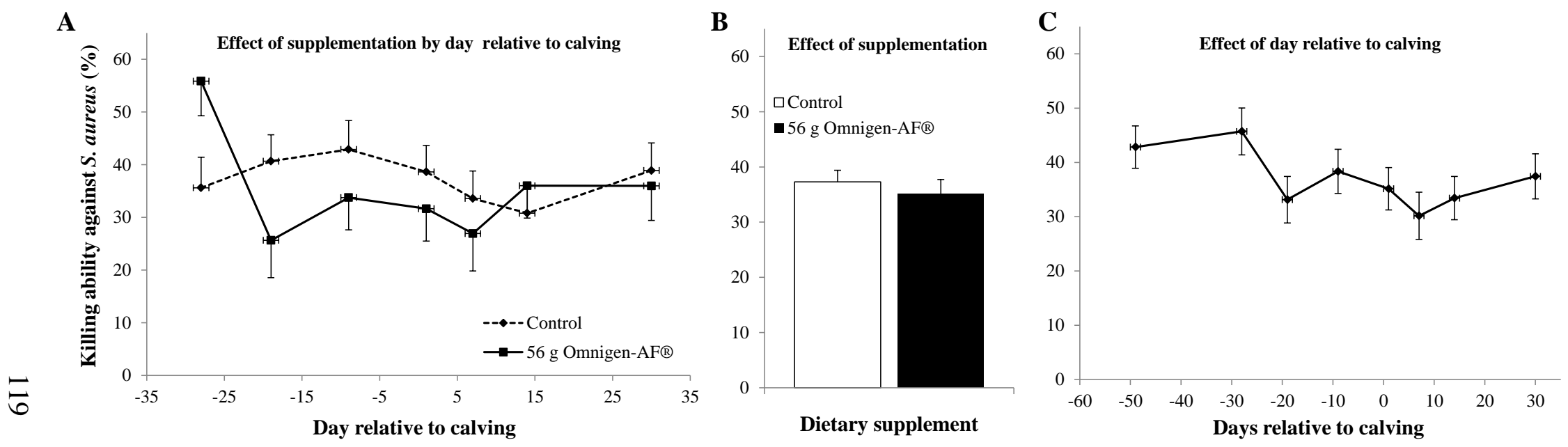

Figure 3.4. Effect of a dietary supplement containing B-complex vitamins and yeast on the neutrophil (PMNL) killing ability against Staphylococcus aureus. Cows received 56 g/day of either OmniGen-AF ${ }^{\circledR}(\mathrm{n}=8)$ or sham control (soybean hulls; $\mathrm{n}$ $=12$ ) mixed into total-mixed rations from day $46 \pm 1$ before calving until day 31 after parturition. PMNL were collected from the blood of cows on days $49 \pm 2$ (pre-treatment sample), $28 \pm 1,19 \pm 1$, and $9 \pm 1$ prepartum and 1, 7, 14, and 30 postpartum. The ability of PMNL to kill S. aureus was determined by calculating the difference in the number of bacteria incubated in the absence and presence of PMNL and dividing this difference by the number of bacteria incubated in the absence of PMNL. (A) Effect of interaction of OmniGen-AF ${ }^{\circledR}$ supplementation by time on the PMNL killing ability against S. aureus, $P=0.096$. (B) Main effect of OmniGen- $\mathrm{AF}^{\circledR}$ supplementation on the PMNL killing ability against S. aureus, $P=0.522$. (C) Main effect of time on the PMNL killing ability against $S$. aureus, $P=0.148$. Data represent adjusted least square means. Error bars indicate the standard error of the mean. ${ }^{\mathrm{a}, \mathrm{b}}$ Means with different letters differ at $P<0.05$ (Tukey). 


\section{DISCUSSION}

In the present study, no effects of a dietary supplement containing B-vitamins, dehydrated yeast, and dried Trichoderma longibrachiatum fermentation products $\left(\right.$ OmniGen- $\left.\mathrm{AF}^{\circledR}\right)$ on the functional capacity of blood neutrophils were detected. There was no effect of OmniGen- $\mathrm{AF}^{\circledR}$ supplementation on the production of ROS, release of NETs, chemotaxis or killing of $S$. aureus by PMNL. Hence, our results suggest that the nutritional supplement OmniGen- $\mathrm{AF}^{\circledR}$ does not improve the ability of the PMNL to eliminate pathogens based on our independent in vitro assays. Other investigators (Wang et al.; 2007, 2009b) have reported immune-modulatory effects of dietary OmniGen-AF ${ }^{\circledR}$ on blood PMNL from sheep and dairy cows. Wang et al. (2007) reported that dexamethasone-immunosuppressed sheep fed OmniGen- $\mathrm{AF}^{\circledR}$ for 28 days had a $329 \%$ higher protein concentration of neutrophil L-selectin compared with non-supplemented immunosuppressed animals. In the same study, OmniGen- $\mathrm{AF}^{\circledR}$ augmented the protein level of interleukin-1 $\beta$ (IL-1 $\beta$ ) in PMNL from immunosuppressed sheep fed wheat pellets contaminated with Aspergillus fumigatus, compared with immunosuppressed animals challenged with the mold (Wang et al., 2007). Therefore, feeding the nutritional supplement OmniGen- $\mathrm{AF}^{\circledR}$ may support immune health by restoring the protein concentration of the PMNL adhesion molecule L-selectin and the inflammatory mediator IL-1 $\beta$, at least in this model of immunosuppression. In dairy cows, feeding $56 \mathrm{~g} /$ day of OmniGen- $\mathrm{AF}^{\circledR}$ during the last month prepartum altered the mRNA contents of 18 genes involved in apoptosis, intercellular communication, and interleukin receptor expression in PMNL purified from blood 15 hours after calving (Wang et al., 2009b). Genes found to be up-regulated by these investigators included interleukin-4 receptor- $\alpha$ (IL-4R $\alpha$ ), 
interleukin converting enzyme (ICE), and fibroblast growth factor (aFGF), which suggested that that $\mathrm{OmniGen}-\mathrm{AF}^{\circledR}$ elicits neutrophil responses also in dairy cows. Contrary to these findings, Schramm et al. (2011) reported no effect of OmniGen-AF ${ }^{\circledR}$ supplementation to dairy cows during the periparturient period on milk SCC and mRNA expression of SELL, IL-8, and ICE by blood PMNL collected on days -7, 9, 19 and 28 relative to parturition. As discussed by these authors, the discrepancy between their results and those reported by Wang et al. $(2007,2009 b)$ may be explained by differences in the timing of PMNL collection because immune-modulators may be more effective in periods of greater immunosuppression such as the day of parturition (Burvenich et al., 2007). However, the results in our experiment suggest that OmniGen- $\mathrm{AF}^{\circledR}$ supplementation does not influence most functional activities of PMNL during the immediate postpartum period, including the day after calving.

Perhaps, the immune-modulator OmniGen- $\mathrm{AF}^{\circledR}$ improves other aspects of cellular mediated immunity that ultimately increase the capacity of mammary gland defense mechanisms to clear the infection. Indeed, water-soluble vitamins such as vitamin B6, vitamin B12, and folic acid influence adaptive immune responses in humans and animal models (Grimble, 1997; Wintergerst et al., 2007). For instance, healthy women consuming a constant diet who received $2.1 \mathrm{mg} / \mathrm{day}$ of the vitamin $\mathrm{B} 6$ pyridoxine hydrochloride for 2 weeks had a 35\% increase in ex vivo lymphocyte proliferation in response to a mitogen, compared to subjects receiving $1 \mathrm{mg} /$ day of vitamin B6 (Kwak et al., 2002). Compared with healthy controls, patients with vitamin B12 deficiency anemia had diminished number of cytotoxic T-lymphocytes, natural killer cell activity, and abnormally high CD4/CD8 ratio which recuperated to normal levels after a methyl-B12 
injection (Tamura et al., 1999). Also, human lymphocytes grown in folic acid-deficient medium had increased DNA strand breakage and overall reduced proliferation, compared to medium supplemented with increasing concentrations of folic acid, suggesting that folate is necessary for DNA stability and growth of lymphocytes (Duthie and Hawdon, 1998). Although the role of these vitamins on adaptive immune function has not been investigated in dairy cows, it is likely that these micronutrients support immunity via similar mechanisms. Furthermore, as reviewed by Suttle and Jones (1989), in vitro tests of immune function may be a better indicator of nutritional status and experiments involving challenge infections may be more appropriate to determine the effects of trace elements on disease resistance. Indeed, Rowson et al. (2011) reported that OmniGen-AF ${ }^{\circledR}$ fed to lactating mice for two weeks prior to experimental intramammary infection with $E$. coli, S. aureus, and S. uberis resulted in reduced DNA accumulation of all three pathogens compared with the glands of challenged rodents receiving no supplementation, suggesting that OmniGen- $\mathrm{AF}^{\circledR}$ is effective in increasing the ability of the host to clear bacterial infections, at least in a rodent model of mastitis.

Regardless of dietary OmniGen- $\mathrm{AF}^{\circledR}$ supplementation, the amount of ROS produced by PMNL increased between days 49 and 19 prepartum, decreased to lowest levels on days 1,7 , and 14 postpartum, and did not return to basal levels until day 30 after calving. Although the timing of blood sampling differs between studies, researchers have consistently reported that PMNL produce the lowest amount of ROS during the first week postpartum which does not recover until 3 or 4 weeks after parturition (Kehrli and Goff, 1989; Kehrli et al., 1989; Cai et al., 1994; Detilleux et al., 1995; Dosogne et al., 1999; Hoeben et al., 2000; Mehrzad et al., 2001b; Revelo and Waldron, 2010; Stevens et 
al., 2011). In agreement with our results, a transient increase in ROS production prior to week 1 prepartum has been also reported by Detilleux et al. (1995) and Dosogne et al. (1999), although the biological meaning of this event is unclear. Ineffective production of ROS by PMNL in the postpartum period has been associated with increased severity of experimentally induced E. coli mastitis (Heyneman et al., 1990). Thus, inability of PMNL to produce adequate amounts of ROS likely contributes to altered immune function in the periparturient period in dairy cows (Burvenich et al., 2007).

NETs release by PMA-stimulated PMNL decreased between days 49 and 28 prepartum, independent of dietary supplementation. No further differences in PMNL expression of NETs were detected between days 28, 19, 9 prepartum and 1, 7, 14 and 30 postpartum in the current report. These results suggest that the ability of the PMNL to release NETs is reduced throughout the periparturient period, at least compared with day 48 prepartum. Also, PMNL tended $(P=0.063)$ to release more NETs on day 30 postpartum, compared with day 19 prepartum suggesting a slight recovery in NETs expression as the cow leaves the periparturient period. In a previous study, we reported that PMNL collected from cows on day 12 prepartum had a 36 and $23 \%$ increase in the release of NETs compared with days 7 and 253 postpartum, respectively (Revelo and Waldron, 2010). The reason why NETs release was not improved in the prepartum period (e.g. day 9 prior to calving) in the current study is unclear, but it is possible that the expression of NETs varies substantially during the days around parturition. Although research is needed to establish the role of NETs in periparturient immunosuppresion, evidence indicates that the antimicrobial activity of NETs released by PMNL may be strategic during immunosuppresion. For example, Grinberg et al. (2008) reported that 
pre-incubation of PMNL with increasing concentrations of beta-hydroxybutyrate inhibited the formation of NETs, phagocytosis and extracellular killing of E. coli which may explain why ketotic cows are more susceptible to infectious diseases. Additionally, incubation of PMNL in milk does not reduce their ability to release NETs, in contrast with other features of neutrophil function, suggesting that when other PMNL killing mechanisms are impaired, neutrophils might rely on killing of bacteria by NETs (Lippolis et al., 2006b).

No changes in PMNL chemotaxis towards IL-8 between any of the sampling periods were detected in this study, regardless of dietary treatment. Previous research in which PMNL chemotaxis was assessed during the periparturient period has yielded conflicting results. In agreement with our findings, several investigators have reported no changes in directed PMNL chemotaxis during the 5 weeks around parturition in healthy cows (Kehrli and Goff, 1989; Cai et al., 1994; Detilleux et al., 1995). In cows with retained placenta, Cai et al. (1994) reported a marked decrease in directed chemotaxis during the first week after parturition, but no change in PMNL chemotaxis in healthy animals. Interestingly, Kimura et al. (2002b) measured PMNL chemotaxis towards supernatant from fetal cotyledon tissue and reported that cows that developed retained placenta had lower chemotaxis than PMNL from healthy cows, particularly during the first week after parturition. These results suggest that compromised PMNL chemotaxis can predispose the dairy cows to develop retained placenta, although alterations in directed PMNL chemotaxis in healthy animals during the periparturient period have been difficult to establish. Recently, Stevens et al. (2011) reported lower PMNL chemotaxis towards complement C5a on day 3 postpartum, compared with PMNL collected from 
cows on day 150 postpartum. Perhaps, the ability of PMNL to migrate towards a chemoattractant decreases as the dairy cow transitions between midlactation into the prepartum period, but does not change around the time of parturition.

The killing ability of PMNL against $S$. aureus after a 60-min co-incubation with the pathogen also did not change between days 49,28, 19, 9 prepartum and 1, 7, 14 and 30 postpartum in this study. Similarly to the PMNL chemotaxis, findings regarding the antimicrobial ability of bovine PMNL during the periparturient period are contradictory. Some studies have found no change in the antibody-mediated cytotoxicity of PMNL (Kehrli et al., 1989; Cai et al., 1994) whereas others have reported a decrease in the antimicrobial ability of PMNL during the first 2 weeks after parturition, compared with the few weeks before calving (Kehrli and Goff, 1989; Daniel et al., 1991; Detilleux et al., 1995). Alternatively, our group has reported that the killing capacity of PMNL against $S$. aureus is higher on day 7 postpartum, compared with the prepartum and midlactation periods (Revelo and Waldron, 2010). As discussed by Detilleux (2004), differences in methodologies used to determine the antimicrobial capacity of PMNL could explain the inconsistency of results relating to changes in the antimicrobial capacity of PMNL during the periparturient period.

\section{CONCLUSIONS}

Dietary supplementation of dairy cows with $56 \mathrm{~g} /$ day of a dietary supplement containing B-vitamins, dehydrated yeast, and dried Trichoderma longibrachiatum fermentation products, commercially available as OmniGen-AF ${ }^{\circledR}$, had no effects on the functional capacity of PMNL during the periparturient period. There were no differences 
in the amount of ROS produced by the PMNL, NETs release, chemotaxis and killing of S. aureus between PMNL collected from OmniGen- $\mathrm{AF}^{\circledR}$-supplemented and nonsupplemented cows. These results suggest that the nutritional supplement OmniGen-AF ${ }^{\circledR}$ does not improve most aspects of PMNL function related to their ability to eliminate pathogens, at least in vitro. Regardless of dietary supplementation, the amount of ROS produced by PMNL increased between days 49 and 19 prepartum, decreased to lowest levels on days 1,7 , and 14 postpartum, and did not recuperate until day 30 after calving. The release of NETs by PMNL decreased between days 49 and 28 prepartum, but no differences in PMNL expression of NETs were detected between days 28, 19, 9 prepartum and 1, 7, 14 and 30 postpartum. Finally, no changes in PMNL chemotaxis towards IL-8 and killing of $S$. aureus during the periparturient period were detected in this study. Reduced ability of PMNL to produce ROS and release NETs during the postpartum period may contribute to the increased incidence and severity of IMI during the transition period in dairy cows. 


\title{
CHAPTER FOUR
}

\section{EFFECT OF A B-VITAMIN AND FUNGAL DIETARY SUPPLEMENT ON THE GLOBAL GENE EXPRESSION PROFILE OF NEUTROPHILS HARVESTED FROM PERIPARTURIENT DAIRY COWS}

\begin{abstract}
The objective of this study was to investigate the effects of a dietary supplement containing B-vitamins, dehydrated yeast, and Trichoderma longibrachiatum fermentation products on the global gene expression profile of neutrophils (PMNL) from periparturient dairy cows. Cows received 56 g/day of either the supplement OmniGen- $\mathrm{AF}^{\circledR}(\mathrm{n}=5)$ or sham control (soybean hulls; $\mathrm{n}=5$ ) mixed into total-mixed rations from day $46 \pm 1$ before calving until day 31 after parturition. PMNL were collected on day 7 postpartum and incubated with 0 or $50 \mu \mathrm{g} / \mathrm{mL}$ of Escherichia coli lipopolysaccharide (LPS) for 120 min. Messenger RNA (poly-A enriched) was purified from the PMNL and converted into a library of template molecules for high throughput RNA-sequencing. Sequenced fragments were then mapped to the bovine reference genome. The resulting digital count data was analyzed to determine the effect of OmniGen- $\mathrm{AF}^{\circledR}$ supplementation on the PMNL global gene expression profile. Feeding OmniGen-AF ${ }^{\circledR}$ to periparturient dairy cows resulted in the differential regulation of 43 genes ( $7 \uparrow$ and $36 \downarrow)$ in PMNL upon activation with LPS. Functional annotation analysis indicated that the lysosome pathway was enriched by the genes with down-regulated expression due to OmniGen-AF ${ }^{\circledR}$ supplementation. Independent from LPS activation, OmniGen-AF ${ }^{\circledR}$ altered the expression of 53 transcripts ( $12 \uparrow$ and $41 \downarrow$, relative to non-supplemented controls). Pathway analysis
\end{abstract}


revealed that genes with down-regulated expression enriched the oxidative phosphorylation pathway. Detected enriched GO terms related to this downregulated pathway included oxidation-reduction, electron transport chain, mitochondrial electron transport, ATP synthesis coupled electron transport, and cellular respiration. These results point out potential molecular mechanisms by which the dietary supplement OmniGen- $\mathrm{AF}^{\circledR}$ may influence the performance of PMNL in periparturient dairy cows. In addition, the effect of PMNL incubation with $50 \mu \mathrm{g} / \mathrm{mL}$ of LPS was also tested independent of dietary supplementation. Incubation with LPS altered the expression of 333 genes $(211 \uparrow$ and $122 \downarrow$, relative to $0 \mu \mathrm{g} / \mathrm{mL})$, independent of dietary treatment. LPS activation in PMNL resulted in the enrichment of the TOLL-like receptor (TLR) signaling and hematopoietic cell lineage pathways. These results may contribute to the understanding of the mechanisms involved in LPS-mediated activation of PMNL. 


\section{INTRODUCTION}

Polymorphonuclear leukocytes or neutrophils (PMNL) are an essential component of the innate immune system whose primary purpose as professional phagocytes is to eliminate invading microbes (Paape et al., 2003). In dairy cows, the successful resolution of intramammary infections depends particularly on the migration of PMNL into the mammary gland and subsequent antimicrobial activity (Vangroenweghe et al., 2005). These PMNL are frequently described as short-lived and terminally differentiated effector cells with limited transcriptional capabilities. Despite this misconception, global gene expression analyses have revealed that human PMNL transcribe a wide range of genes involved in phagocytosis, respiratory burst activity, and cytokine synthesis (Newburger et al., 2000). In dairy cows, however, only a few studies have aimed to investigate the global genetic profile of bovine PMNL. For instance, Madsen et al. (2002) hypothesized that parturition induces changes in the gene expression of PMNL that may result in the defective immune cell phenotype typical of the periparturient period. These investigators used differential display PCR to measure mRNA contents in PMNL during the periparturient period and reported a decreased expression of eight genes, including cytochrome $b$ and rig/ribosomal protein $\mathrm{S} 15$. The same group then used microarrays containing 1,056 cDNA probes to further identify the changes in gene expression during the weeks around parturition and reported that 42 genes had altered expression of at calving, 14\% of which encode proteins involved in cell apoptosis (Madsen et al., 2004). These authors noted that the expression of genes encoding for proteins that induce apoptosis was strongly diminished at parturition whereas pro-survival genes were upregulated. Additional genes found to be differentially expressed due to the stress of 
parturition include those involved in the activation of the immune response and tissue remodeling (Burton et al., 2005). These functional genomic studies using PCR and microarrays have shed light into some of the molecular mechanisms underlying the effects of parturition on immune function.

The development of novel high-throughput sequencing technologies has provided a new method for quantifying global transcript expression levels termed RNA-sequencing or RNA-seq (Mortazavi et al., 2008). Although RNA-seq technology is still under development, it has several advantages over hybridization-based methods. First, RNAseq does not rely on pre-existing assumptions about the genome sequence. Second, digital transcript-counting results in a very low, if any, background signal. Finally, RNA-seq offers great sensitivity for transcripts expressed at low or very high levels (Wang et al., 2009a). Indeed, a direct comparison between RNA-seq and microarray technology has shown improved performance in gene expression analysis conducted with RNA-seq (Sultan et al., 2008). These researchers reported that using RNA-seq during transcriptome analysis of human embryonic kidney and B cells resulted in the elucidation of at least $25 \%$ more genes, compared with microarrays. As a result of improved performance and less limitations compared with hybridization-based technologies, RNA-seq methods are now in common use in biology (Cullum et al., 2011).

In dairy cows, the incidence and severity of clinical mastitis increase during the periparturient period due in part to an incompetent immune system (Mallard et al., 1998). It has been proposed that one of the underlying causes of this periparturient immunosuppression may be the inadequate amounts of dietary vitamins and trace minerals (Goff, 2006; Spears and Weiss, 2008). Although synthesis by rumen microbes 
should provide considerable amounts of B-vitamins, limited research has been conducted to determine the dairy cow requirements for B-vitamins to support gestation, milk production and health (NRC, 2001). Furthermore, dietary supplementation with a blend of B-vitamins, dehydrated yeast, and dried Trichoderma longibrachiatum fermentation products (OmniGen-AF ${ }^{\circledR}$, Prince Agri Products, Inc. Quincy, IL) has been reported to influence the immune responses of dairy cows (Wang et al., 2007; 2009b). Wang et al. (2009b) used an array containing approximately 1,500 sequenced tag clones to test the hypothesis that dietary supplementation with OmniGen- $\mathrm{AF}^{\circledR}$ would influence the expression of genes involved in the PMNL immune response. In this study, OmniGen$\mathrm{AF}^{\circledR}$ supplementation caused greater expression of 12 genes and decreased expression of six genes related to immune function. These authors reported that the dietary supplement induced changes in PMNL in the expression of genes involved in apoptosis, cell-to-cell communication, and intracellular signaling (Wang et al., 2009b). Although these findings provided evidence that OmniGen- $\mathrm{AF}^{\circledR}$ may have immune-modulatory effects via changes in gene expression in PMNL, the use of a microarray limited the discovery of genes differentially expressed to the number of cDNA probes present in the array. Therefore, the objective of this study was to use RNA-seq to investigate the effects of the dietary supplement OmniGen- $\mathrm{AF}^{\circledR}$ on the global gene expression profile of resting and LPSactivated neutrophils harvested from periparturient dairy cows. It was hypothesized that (1) feeding the dietary supplement OmniGen- $\mathrm{AF}^{\circledR}$ would alter the expression of genes in PMNL towards an enhanced immune response; and (2) LPS would cause stimulate the transcription of genes encoding for proteins involved in immune activation. 


\section{MATERIALS AND METHODS}

\section{Experimental Design, Animals, and Dietary Treatments}

The use and care of all animals were approved by The University of Missouri Animal Care and Use Committee. All cows enrolled in the study did not experience any major clinical event during the previous lactation, had an average previous lactation somatic cell count lower than 300,000 cells/ml and a current locomotion score lower than two (Sprecher et al., 1997). Ten cows received $56 \mathrm{~g} /$ day of either OmniGen- $\mathrm{AF}^{\circledR}(\mathrm{n}=5)$ or sham control (soybean hulls; $\mathrm{n}=5$ ) mixed into total-mixed rations from day $46 \pm 1$ before calving until day 31 after parturition. OmniGen- $A F^{\circledR}$ is a dietary supplement manufactured by Prince Agri Products, Inc. (Quincy, IL) that contains a mixture of Bcomplex vitamins, dehydrated yeast, and dried Trichoderma longibrachiatum fermentation products (Table 4.1). All cows were fed diets formulated to meet or exceed nutritional recommendations for all nutrients (Table 4.2; NRC, 2001). Cows were housed in a free-stall barn, milked twice daily and fed for ad libitum feed intake via Calan doors. Treatment groups were balanced for parity, mature equivalent 305 milk production, and body condition score. To determine the effect of the dietary supplement OmniGen-AF ${ }^{\circledR}$ on the genetic profile of circulating PMNL, approximately $450 \mathrm{~mL}$ of blood were collected on day 7 after parturition. Blood was collected via jugular vein catheters into sterile $500 \mathrm{~mL}$ polypropylene conical tubes (Corning Inc., NY) containing $75 \mathrm{~mL}$ of acidcitrate dextrose, inverted three times. Catheters were inserted using aseptic techniques and immediately removed after collection of samples. Blood samples were immediately stored on ice until isolation of blood PMNL ( $<20 \mathrm{~min})$. 
Table 4.1. Composition of the dietary supplement Omnigen-AF ${ }^{\circledR}$

\begin{tabular}{lcc}
\hline Nutrients $^{1}$ & Concentration, $\mathrm{mg} / \mathrm{kg}$ & ${\text { Supplemented, } \mathrm{mg} / \mathrm{day}^{2}}^{2}$ \\
\hline Vitamin B-12 & 198.4 & 11.11 \\
Riboflavin & 14.3 & 0.80 \\
D-pantothenic acid & 152.1 & 8.52 \\
Thiamin & 75.0 & 4.20 \\
Niacin & 496.0 & 27.78 \\
Vitamin B-6 & 44.1 & 2.47 \\
Folic acid & 6.6 & 0.37 \\
Choline & 77.2 & 4.32 \\
Biotin & 2.2 & 0.12 \\
\hline
\end{tabular}

Omnigen- $\mathrm{AF}^{\circledR}$ is manufactured by Prince Agri Products, Inc. (Quincy, IL).

${ }^{1}$ Ingredients: Hydrated sodium calcium aluminosilicate, diatomaceous earth, brewers dehydrated yeast, mineral oil, calcium carbonate, dried Trichoderma longibrachiatum fermentation products, rice hulls, niacin supplement, biotin, d-calcium pantothenate, vitamin B-12 supplement, choline chloride, thiamine mononitrate, pyridoxine hydrochloride, silicon dioxide, riboflavin-5-phosphate and folic acid.

${ }^{2}$ Cows in the supplemented group received $56 \mathrm{~g} /$ day of OmniGen-AF ${ }^{\circledR}(\mathrm{n}=5)$. Animals in the control group were fed $56 \mathrm{~g}$ /day of soybean hulls $(\mathrm{n}=5)$. 
Table 4.2. Chemical composition of the prepartum and postpartum diets (DM basis)

\begin{tabular}{|c|c|c|}
\hline Nutrient & Prepartum diet ${ }^{1,2}$ & Postpartum diet ${ }^{1,2}$ \\
\hline Crude Protein, $\%$ & 12.6 & 17.4 \\
\hline Available Protein, $\%$ & 11.5 & 16.1 \\
\hline ADICP, \% & 1.0 & 1.3 \\
\hline Acid Detergent Fiber, \% & 33.5 & 21.1 \\
\hline Neutral Detergent Fiber, \% & 50.3 & 30.6 \\
\hline Crude Fat, \% & 2.2 & 4.4 \\
\hline TDN, \% & 61.7 & 71.8 \\
\hline NEL, Mcal/kg & 1.4 & 1.7 \\
\hline NEm, Mcal/kg & 1.3 & 1.7 \\
\hline $\mathrm{NEg}, \mathrm{Mcal} / \mathrm{kg}$ & 0.7 & 1.1 \\
\hline Soluble Protein, \% CP & 20.2 & 26.3 \\
\hline Lignin, \% & 4.7 & 4.2 \\
\hline NDICP, \% & 4.1 & 5.2 \\
\hline Starch, \% & 13.5 & 27.4 \\
\hline $\mathrm{NFC}, \%$ & 30.7 & 44.7 \\
\hline Calcium, \% & 0.86 & 1.00 \\
\hline Phosphorus, \% & 0.23 & 0.28 \\
\hline Magnesium, \% & 0.38 & 0.36 \\
\hline Potassium, \% & 1.36 & 1.39 \\
\hline Sodium, \% & 0.10 & 0.33 \\
\hline Iron, ppm & 336.0 & 335.7 \\
\hline Zinc, ppm & 77.2 & 100.2 \\
\hline Copper, ppm & 22.2 & 28.2 \\
\hline Manganese, ppm & 77.7 & 81.0 \\
\hline Molybdenum, ppm & 1.3 & 1.4 \\
\hline Cobalt, ppm & 0.1 & 0.2 \\
\hline Sulfur, \% & 0.22 & 0.25 \\
\hline Ash, $\%$ & 8.4 & 8.0 \\
\hline
\end{tabular}

${ }^{1}$ Diets were formulated to meet or exceed nutritional recommendations for all nutrients (NRC, 2001).

${ }^{2}$ Values represent averages of samples composited every $4 \mathrm{wk}$. 


\section{Isolation of Bovine Neutrophils}

Neutrophils were isolated using procedures modified from Weber et al. (2001) as validated in our laboratory. The $500 \mathrm{~mL}$ conical tubes (Corning Inc.) containing the blood sample were centrifuged at $1000 \times g$ for 20 min at $4^{\circ} \mathrm{C}$ to separate plasma from the cell pack. Plasma was discarded and the remaining cells were suspended in 1,450 mL of calcium and magnesium-free Hanks' Balanced Salt Solution (CMF-HBSS; Sigma Chemical Co.). The suspension was gently mixed and $40 \mathrm{~mL}$ aliquots were pipetted down the side of a $50-\mathrm{mL}$ polypropylene conical tube containing $10 \mathrm{~mL}$ of $1.084 \mathrm{~g} / \mathrm{mL}$ of Percoll $^{\circledR}$ (GE Healthcare Bio-Sciences Corp., Piscataway, NJ). The cells were then subjected to gradient centrifugation $\left(400 \times \mathrm{g}\right.$ for $40 \mathrm{~min}$ at $\left.22^{\circ} \mathrm{C}\right)$. The supernatant, mononuclear cell layer and Percoll ${ }^{\circledR}$ were aseptically aspirated and discarded. The pellet (PMNL and erythrocytes) was retained and erythrocytes were lysed by hypotonic shock. For this procedure, two volumes of an ice-cold $10.56 \mathrm{~m} M \mathrm{Na}_{2} \mathrm{HPO}_{4}, 2.67 \mathrm{mM} \mathrm{NaH} \mathrm{PO}_{4}$ solution were added to one volume of cell suspension and the solution was mixed by gentle aspiration and inversion. Tonicity was restored by the addition of one volume of a $10.56 \mathrm{~m} M \mathrm{Na}_{2} \mathrm{HPO}_{4}, 2.67 \mathrm{~m} M \mathrm{NaH}_{2} \mathrm{PO}_{4} 0.43 M \mathrm{NaCl}$ solution. The cell solution was then centrifuged $(850 \times \mathrm{g})$ for $4 \mathrm{~min}$ at $4^{\circ} \mathrm{C}$ and the pellet washed twice by resuspension in $35 \mathrm{~mL}$ of CMF-HBSS and recentrifugation $\left(850 \times \mathrm{g}\right.$ for $4 \mathrm{~min}$ at $\left.4^{\circ} \mathrm{C}\right)$. Cells were

enumerated and viability was determined by propidium iodide (Sigma Chemical Co.) exclusion using a Cellometer Vision ${ }^{\circledR}$ automatic cell counter (Nexcelom Bioscience LLC., Lawrence, MA). The concentration of cells was adjusted and the suspension was kept at $4{ }^{\circ} \mathrm{C}$ until incubation with LPS. 


\section{Incubation of PMNL with $E$. coli Lipopolysaccharide}

The effects of the dietary supplement on the genetic profile of neutrophils were determined in both resting and LPS-stimulated PMNL. Lipopolysaccharide from E. coli (serotype O55:B5, strain CDC 1644-70) was obtained from Sigma Chemical Co. A working solution of $250 \mu \mathrm{g} / \mathrm{mL}$ LPS was added to $50-\mathrm{mL}$ polypropylene conical tubes (Corning Inc., NY) containing purified neutrophils to reach final concentrations of 0 or $50 \mu \mathrm{g} / \mathrm{mL}$ of LPS at a cell concentration of $1.2 \times 10^{7}$ cells $/ \mathrm{mL}$. The tubes were then placed in a humidified incubator $\left(37^{\circ} \mathrm{C}, 5 \% \mathrm{CO}_{2}\right)$ and incubated for 120 minutes while inverted in a Labquake ${ }^{\circledR}$ tube rotator (Barnstead-Thermolyne Corp., Dubuque, IA). Under similar conditions, our laboratory has tested the effects of increasing concentrations of LPS $(0,1,25$ and $50 \mu \mathrm{g} / \mathrm{mL}$ of LPS) on PMNL function (Revelo and Waldron, 2012), and from that study, concluded that the $50 \mu \mathrm{g} / \mathrm{mL}$ dose would be most effective for the current experiment. After incubation, the cell solutions were centrifuged $(850 \mathrm{x} g$ ) for 4 min at $4^{\circ} \mathrm{C}$. The cell pellet was re-suspended in Trizol $^{\circledR}$ at a concentration of $1 \times 10^{7}$ cells/mL. This suspension was homogenized using a sonic dismembrator (Sonifier ${ }^{\circledR}$, Branson Ultrasonics Corp., Danbury, CT) and stored at $-80^{\circ} \mathrm{C}$ until mRNA extraction.

\section{mRNA Extraction}

Total cellular RNA was harvested from neutrophils using $1 \mathrm{~mL}$ of Trizol ${ }^{\circledR}$ reagent (Invitrogen Co., Carlsbad, CA) per 1 x $10^{7}$ cells, according to the manufacturer recommendations. Messenger RNA was further purified using the RNeasy ${ }^{\circledR}$ Mini Kit (Qiagen, Valencia, CA). Genomic DNA was then removed by on-column DNase digestion using the RNase-Free DNase Set (Qiagen) during the RNeasy ${ }^{\circledR}$ procedure. The concentration and purity of RNA were determined with a ND-1000 NanoDrop ${ }^{\circledR}$ 
spectrophotometer (NanoDrop Technologies, Wilmington, DE). In addition, the integrity

of RNA was assessed by electrophoresis and fluorescence detection using the Experion ${ }^{\mathrm{TM}}$ automated electrophoresis system (Bio-Rad, Hercules, CA). After purification and quality control, RNA samples were stored at $-80^{\circ} \mathrm{C}$ in RNase-free water.

\section{Library Preparation and mRNA Sequencing}

Messenger RNA was converted into a library of template molecules for high throughput cDNA sequencing (Illumina, Inc., Hayward, CA) by the University of Missouri DNA Core following the manufacturer's recommendations. First, poly-A containing mRNA molecules were purified via poly-T oligo-attached magnetic beads using the TruSeq $^{\circledR}$ RNA sample preparation kit (Illumina, Inc.). Poly-A mRNA molecules were then fragmented into small pieces using divalent cations under high temperatures. Subsequently, first strand cDNA synthesis was performed using reverse transcriptase and random primers followed by second strand cDNA synthesis using DNA polymerase I and RNase H. Next, blunt-end fragments are created and the 3' ends of these cDNA fragments were adenylated. After indexed adapters were ligated, these products were purified and enriched by PCR to create the final cDNA libraries. The amplified libraries were indexed for multiplexing during this step. The size, purity, and concentration of the final constructs were evaluated using the Agilent BioAnalyzer $2100^{\circledR}$ automated electrophoresis system (Agilent Technologies, Inc., Santa Clara, CA) and the Qubit $^{\circledR}$ fluorometer (Invitrogen, Co.) using the Quant-iT ${ }^{\mathrm{TM}}$ HS dsDNA reagent kit (Invitrogen, Co.). The indexed cDNA libraries were pooled into a single lane (six libraries per lane) of a flow cell. Single-read sequencing by synthesis was conducted on the Illumina HiSeq 2000 according to the manufacturer's recommendations. Called 
sequenced reads were approximately 50 bases in length. The resulting data were collected and de-multiplexed using the CASAVA package version 1.8 (Illumina, Inc.) for downstream analysis.

\section{Sequence alignment}

Reads were processed and mapped against a bovine reference genome using the NextGene ${ }^{\circledR}$ sequence analysis software v. 2.15 (SoftGenetics, LLC., State College, PA). First, sequencing output files (FASTQ) were converted to FASTA format. Subsequently, low quality reads were trimmed or removed from the sample file using the Format Conversion tool. Quality thresholds were used to identify low quality reads. Reads were removed from the sample file if they failed to meet one of the following criteria: median quality score $>20,<3$ uncalled bases, $>35$ called bases, and $<3$ consecutive bases with a quality score less than 15 (Table 4.3). Reads that passed the quality filter were then aligned to the Bos_taurus_UMD_3.1 reference assembly (available at $\mathrm{ftp}$ //ftp.ncbi.nih.gov/genomes/Bos_taurus/) using the Sequence Alignment tool. A seeded alignment was carried out using the first 24 bases with four bases between seed start positions. Reads that mapped equally well to more than 50 genomic regions were discarded. For reads that did not match exactly to the reference, a maximum of two mismatched bases were allowed to align the entire read to the genome. Reads with a matching base percentage lower than $85 \%$ were further filtered out. The average counts for matched and unmatched reads are indicated in Table 4.4. After alignment, expression reports were generated using the gene sequences in the reference assembly as the alignment segments. Digital sequencing read counts were obtained from these reports and used in the statistical analysis. 
Table 4.3. Conversion and quality control summary

\begin{tabular}{lc}
\hline Description & Number $^{\mathbf{1}}$ \\
\hline Total sequenced fragments & $32,275,264$ \\
Reads that passed the quality thresholds & $28,421,029$ \\
Reads that failed to pass the quality filter & \\
Median quality score $<20$ & 860,333 \\
Uncalled bases per read > 3 & 34,165 \\
Called bases per read < 35 & 249,737 \\
Three consecutive bases with quality score < 15 & $2,710,000$ \\
Total & $3,854,235$ \\
Reads trimmed due to low base quality score & $6,777,841$ \\
Bases trimmed due to low base quality score & $132,756,242$ \\
Mean read length, bases & 48.74 \\
\hline
\end{tabular}

${ }^{\mathrm{T}}$ Values represent the average of 10 sample files. 
Table 4.4. Summary of sequencing read alignment to the reference bovine genome

\begin{tabular}{|c|c|}
\hline Description $^{1}$ & Number $^{2}$ \\
\hline Total matched reads & $25,110,957$ \\
\hline Perfectly matched reads & $20,591,998$ \\
\hline Total unmatched reads & $3,309,867$ \\
\hline Matched bases & $2,161,869,805$ \\
\hline Unmatched bases recorded as mutations & $3,294,007$ \\
\hline Average read length & 48.2 \\
\hline Average coverage & 7.6 \\
\hline Reference assembly length, bases & $2,670,102,999$ \\
\hline Covered bases & $260,251,637$ \\
\hline Percent coverage ${ }^{3}$ & $9.7 \%$ \\
\hline Percentage of reads mapped ${ }^{4}$ & $88.4 \%$ \\
\hline \multicolumn{2}{|c|}{$\begin{array}{l}{ }^{1} \text { Reads were aligned to the Bos_taurus_UMD_3.1 bovine reference assembly (available } \\
\text { at ftp://ftp.ncbi.nih.gov/genomes/Bos_taurus/). } \\
{ }^{2} \text { Values represent the average of } 10 \text { sample files. } \\
{ }^{3} \text { Covered bases divided by the total bases in bovine genome, multiplied by } 100 \text {. } \\
{ }^{4} \text { Total reads matched divided by the sum of matched and unmatched reads, multiplied by } \\
100 \text {. }\end{array}$} \\
\hline
\end{tabular}




\section{Statistical Analysis}

A modified generalized linear modeling (GLM) approach was used to model the read counts in order to determine which genes were differentially expressed. Traditional GLMs are, as their name implies, extensions of the usual linear model framework (e.g., ANOVA and linear regression) to data that is not normally distributed (non-Gaussian). Several computational modifications and theoretical extensions were made to the traditional GLM framework and its estimation algorithm to make them applicable to high-throughput read count data, as detailed in (McCarthy et al., 2012). These methods are available in the edgeR package (Robinson et al., 2010) available through the opensource Bioconductor/R project. The approach implemented in edgeR (limma-voom pipeline) fitted a separate count model for each gene, but it is done in an empirical Bayes manner which permits a flexible borrowing of information across genes in order to improve the precision of the model estimates. The voom method models the counts after a $\log$ transformation $\left(\log y_{g i}+0.5\right.$, where $g$ is a gene in a sample $i$ ) to converge the variables to normality.

For each analysis, models were fit in edgeR using gene-wise estimates of the dispersion. The design matrix incorporated the main effect of dietary supplementation (OmniGen- $\mathrm{AF}^{\circledR}$ or sham control), LPS incubation $(0$ or $50 \mu \mathrm{g} / \mathrm{mL}$ of LPS), and their interaction. Fixed cow-level effects were also incorporated to account for the dependency of measurements form the same animal. Model fit was assessed through checks for numerical convergence failure, quantile-quantile plots, and goodness-of fit test. To account for pre-existing variation between animals, several measurements of PMNL function obtained from the same cows (Revelo et al., dissertation chapter three) were 
tested as potential covariates. Two of these baseline PMNL functional measurements, reactive oxygen species (ROS) and antimicrobial capacity were used as covariates in the model. To determine which genes were differentially expressed, an overall F-test of three pre-planned contrasts was conducted. To account for multiple testing the $P$-values from the overall F-test were adjusted via the false discovery rate (FDR) approach of Benjamini and Hochberg, which controls the expected FDR. Genes beneath the expected FDR cutoff of $20 \%$ were considered statistically significant. If a gene was significant at a FDR of $20 \%$, then each contrast within that gene was tested. The contrasts in the analysis tested differences within the interaction effect $\left[\left(\mathrm{OmniGen}^{-\mathrm{AF}^{\circledR}}{ }^{\text {50LPS/OmniGen-AF }}{ }^{\circledR}\right.\right.$ OLPS) vs. (control 50LPS/control 0LPS)], main effect of supplementation (OmniGen-AF ${ }^{\circledR}$ vs. control), and main effect of LPS (50LPS vs. OLPS). Percent changes in gene expression were calculated relative to the second term in each contrast.

\section{Functional Annotation Analyses}

Differentially expressed genes were loaded to the DAVID Bioinformatics Resources 6.7 (http://david.abcc.ncifcrf.gov/home.jsp; DAVID Bioinformatics, 2012) for pathway and gene ontology (GO) enrichment analyses as described by Huang et al. (2009b). The functional annotation chart module of DAVID was used to identify enriched annotation terms. Differentially regulated genes within each contrast were mapped to associated biological annotation GO terms. To identify enriched pathways, all genes differentially regulated by the comparison between dietary treatments were used. Enriched pathways were then visualized using the Kyoto Encyclopedia of Genes and Genomes (http://www.genome.jp/keg; KEGG, 2012) and genes classified according to up- or down-regulation. The enrichment of gene members for each of the annotation 
terms (e.g., GO terms and pathways) was tested by a modified Fisher's exact test (EASE score) using the Bos taurus genome as a reference or background. For the GO enrichment analysis, GO terms were limited to biological processes and molecular functions. The thresholds to select significant KEGG pathways and GO categories were EASE score of $<0.15$ and count $>4$.

\section{RESULTS}

\section{Differential gene expression}

On average, 32.3 million sequence fragments were recovered from each sequenced cDNA library. From the 28.4 million fragments that passed the quality thresholds (Table 4.3), 88\% mapped to the bovine reference genome with a percent coverage of $9.7 \%$ (Table 4.4). After covariate adjustment, analysis of variance conducted on the digital counts revealed a total of 429 genes differentially regulated across all contrasts (Supplemental Table 4.6; $P<0.050$, corrected for multiple testing at an FDR of $20 \%$ ). For those genes that showed differential expression in any of the contrast (overall F-test; $P<0.05,20 \%$ FDR) its statistical significance within each contrast was tested. In the first contrast, the differential effect of LPS (50 vs. $0 \mu \mathrm{g} / \mathrm{mL}$ ) was compared between PMNL harvested from OmniGen-AF ${ }^{\circledR}$ - and non-supplemented cows. Supplementing cows with OmniGen- $\mathrm{AF}^{\circledR}$ resulted in the differential regulation of 43 genes ( $7 \uparrow$ and $\left.36 \downarrow\right)$ in PMNL after activation with LPS (Supplemental Table 4.6A; $P<0.050,20 \%$ FDR). The top five up-regulated genes within this contrast included uncharacterized LOC100298870 (LOC100298870), cholinergic receptor, nicotinic, alpha 6 (CHRNA6), T-cell activation Rho GTPase-activating protein-like (LOC100337120), nuclear receptor 
subfamily two, group E, member one (NR2E1), and leukocyte cell derived chemotaxin one (LECT1). In contrast, the top five genes with down-regulated expression were sorting nexin family member 30 (SNX30), leptin receptor overlapping transcript (LEPROT), tumor protein D52-like 2 (TPD52L2), surfeit 6 (SURF6), and adaptor-related protein complex two, mu one subunit (AP2M1).

Regardless of LPS activation, feeding OmniGen- $\mathrm{AF}^{\circledR}$ to dairy cows resulted in the differential regulation of 53 transcripts $(12 \uparrow$ and $41 \downarrow)$, relative to non-supplemented animals (Supplemental Table 4.6B; $P<0.050,20 \%$ FDR). The top five up-regulated genes for the main effect of OmniGen- $\mathrm{AF}^{\circledR}$ supplementation included histone H3-like centromeric protein A-like (LOC782601), patr class I histocompatibility antigen (LOC788634), agrin (AGRN), tumor necrosis factor receptor superfamily member 17 (TNFRSF17), and RNA terminal phosphate cyclase-like 1 (RCL1). The top five downregulated genes for the main effect of OmniGen- $\mathrm{AF}^{\circledR}$ supplementation included eukaryotic translation initiation factor 4E binding protein 1(EIF4EBP1), chromosome eight open reading frame (C8H9orf72), UDP-N-acetyl-alpha-Dgalactosamine:polypeptide acetylgalactosaminyltransferase 11 (GALNT11), endoplasmic reticulum aminopeptidase 2 (ERAP2), and uncharacterized LOC515828 (LOC515828).

Incubation of PMNL with $50 \mu \mathrm{g} / \mathrm{mL}$ of LPS altered the expression of 333 genes ( $211 \uparrow$ and $122 \downarrow$, relative to $0 \mu \mathrm{g} / \mathrm{mL}$ ), independent of dietary treatment (Supplemental Table 4.6C; $P<0.050,20 \%$ FDR). The top five up-regulated genes for the main effect of LPS supplementation included secreted phosphoprotein 1 (SPP1), interleukin (IL)-6 , ras homolog gene family, member C (RHOC), serum amyloid A1 (SAA1), and serum amyloid A 3 (SAA3). The top five down-regulated genes for the main effect of LPS 
supplementation included chromosome 7 open reading frame (C7H19orf71), transmembrane protein 79 (TMEM79), lymphoblastic leukemia derived sequence 1 (LYL1), chromosome 3 open reading frame, human C1orf85 (C3H1orf85), and SAYSVFN motif domain containing 1 (SAYSD1).

\section{Pathway and gene ontology enrichment analysis}

Pathway analysis was performed to identify enriched biological pathways in PMNL due to OmniGen-AF ${ }^{\circledR}$-supplementation and LPS treatment. A total of four enriched pathways were detected across all contrasts. In the comparison of the LPS effect between PMNL harvested from OmniGen- $\mathrm{AF}^{\circledR}{ }_{-}$and non-supplemented cows, the lysosome pathway was found to be enriched by genes with down-regulated expression in the differential gene expression analysis (Figure 4.1, $P=0.034$ ). Furthermore, the oxidative phosphorylation pathway was altered in response to OmniGen- $\mathrm{AF}^{\circledR}$ supplementation, regardless of PMNL incubation with LPS. Similarly to the lysosome pathway, the genes responsible for the enrichment of this pathway were down-regulated (Figure 4.2, $P<0.001$ ). Finally, LPS activation resulted in the enrichment of the TOLLlike receptor (TLR) signaling (Figure 4.3, $P=0.038$ ) and hematopoietic cell lineage (Figure 4.4, $P=0.089$ ) pathways, independent of dietary supplementation. These pathways were enriched by genes with up-regulated expression in response to LPS. 
Figure 4.1. Effect of supplementing cows with OmniGen-AF ${ }^{\circledR}$ on the lysosome pathway in neutrophils (PMNL) after activation with Escherichia coli lipopolysaccharide (LPS). Cows received $56 \mathrm{~g} /$ day of either OmniGen-AF ${ }^{\circledR}(\mathrm{n}=5)$ or sham control (soybean hulls; $n=5$ ) mixed into total-mixed rations from day $46 \pm 1$ before calving until day 31 after parturition. PMNL were collected from the blood of cows on day 7 postpartum and then incubated with 0 or $50 \mu \mathrm{g} / \mathrm{mL}$ of Escherichia coli lipopolysaccharide (LPS) for 120 min to determine their global gene expression using RNA-sequencing. Genes with altered expression due to OmniGen-AF ${ }^{\circledR}$ supplementation (relative to non-supplemented controls) and incubation with $50 \mu \mathrm{g} / \mathrm{mL}$ of LPS (relative to $0 \mu \mathrm{g} / \mathrm{mL}$ of LPS) were loaded to DAVID Bioinformatics Resources 6.7 where KEGG pathway analysis was applied to uncover enriched functional pathways. Objects highlighted in red represent downregulated gene products. Green boxes correspond to gene products specific for the Bos Taurus genome that were unaltered by treatments. Significance of gene-pathway enrichment conducted with a modified Fisher's exact test (EASE score, $P=0.034$ ). 


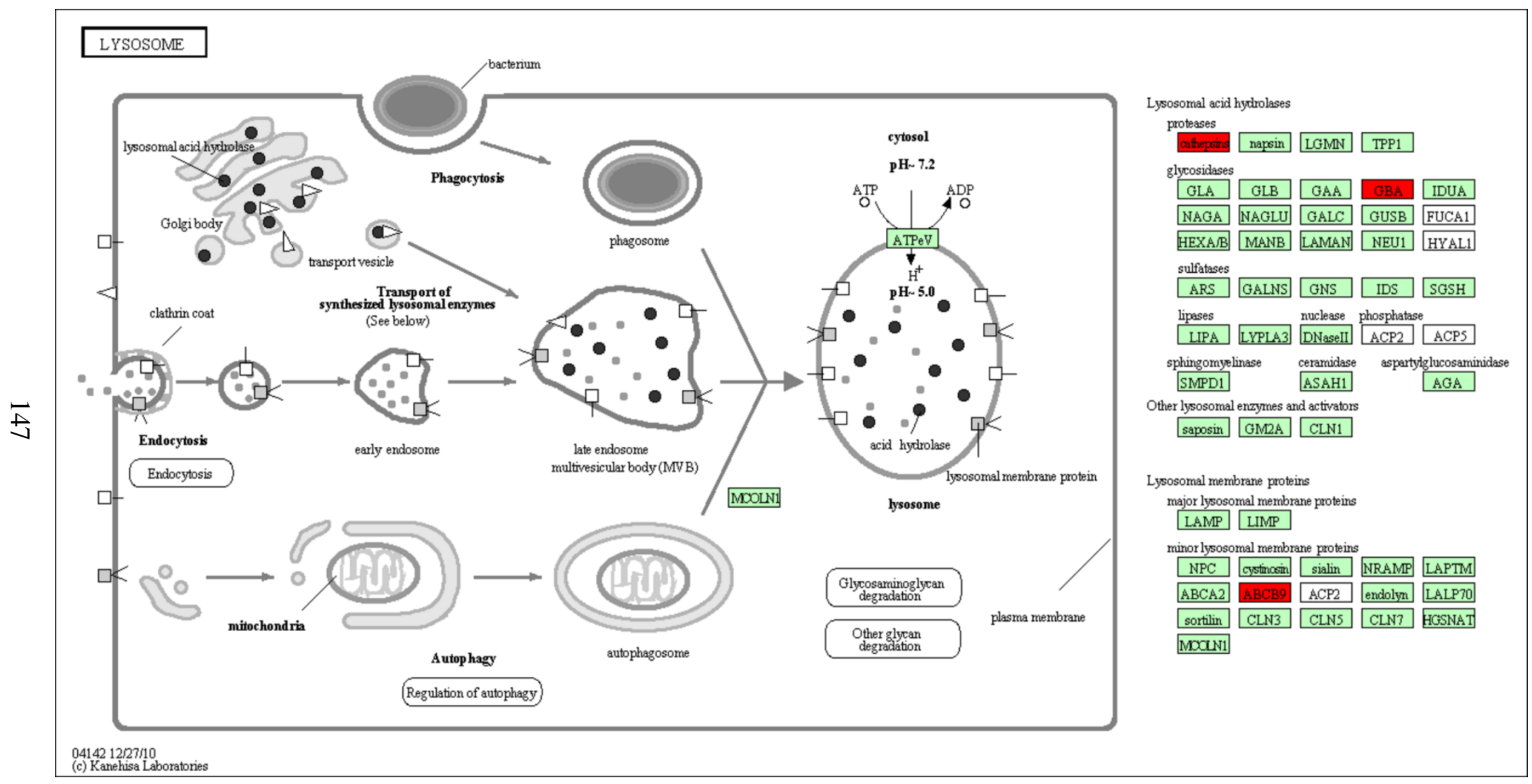


Figure 4.2. Effect of supplementing cows with OmniGen-AF ${ }^{\circledR}$ on the oxidative phosphorylation pathway in neutrophils (PMNL) regardless of incubation with Escherichia coli lipopolysaccharide (LPS). Cows received 56 g/day of either OmniGen$\mathrm{AF}^{\circledR}(\mathrm{n}=5)$ or sham control (soybean hulls; $\mathrm{n}=5$ ) mixed into total-mixed rations from day $46 \pm 1$ before calving until day 31 after parturition. PMNL were collected from the blood of cows on day 7 postpartum and then incubated with 0 or $50 \mu \mathrm{g} / \mathrm{mL}$ of Escherichia coli lipopolysaccharide (LPS) for $120 \mathrm{~min}$ to determine their global gene expression using RNA-sequencing. Genes with altered expression due to OmniGen-AF ${ }^{\circledR}$ supplementation (relative to non-supplemented controls) were loaded to DAVID Bioinformatics Resources 6.7 where KEGG pathway analysis was applied to uncover enriched functional pathways. Objects highlighted in red represent down-regulated gene products. Green boxes correspond to gene products specific for the Bos Taurus genome that were unaltered by treatments. Significance of gene-pathway enrichment conducted with a modified Fisher's exact test (EASE score, $P<0.001$ ). 


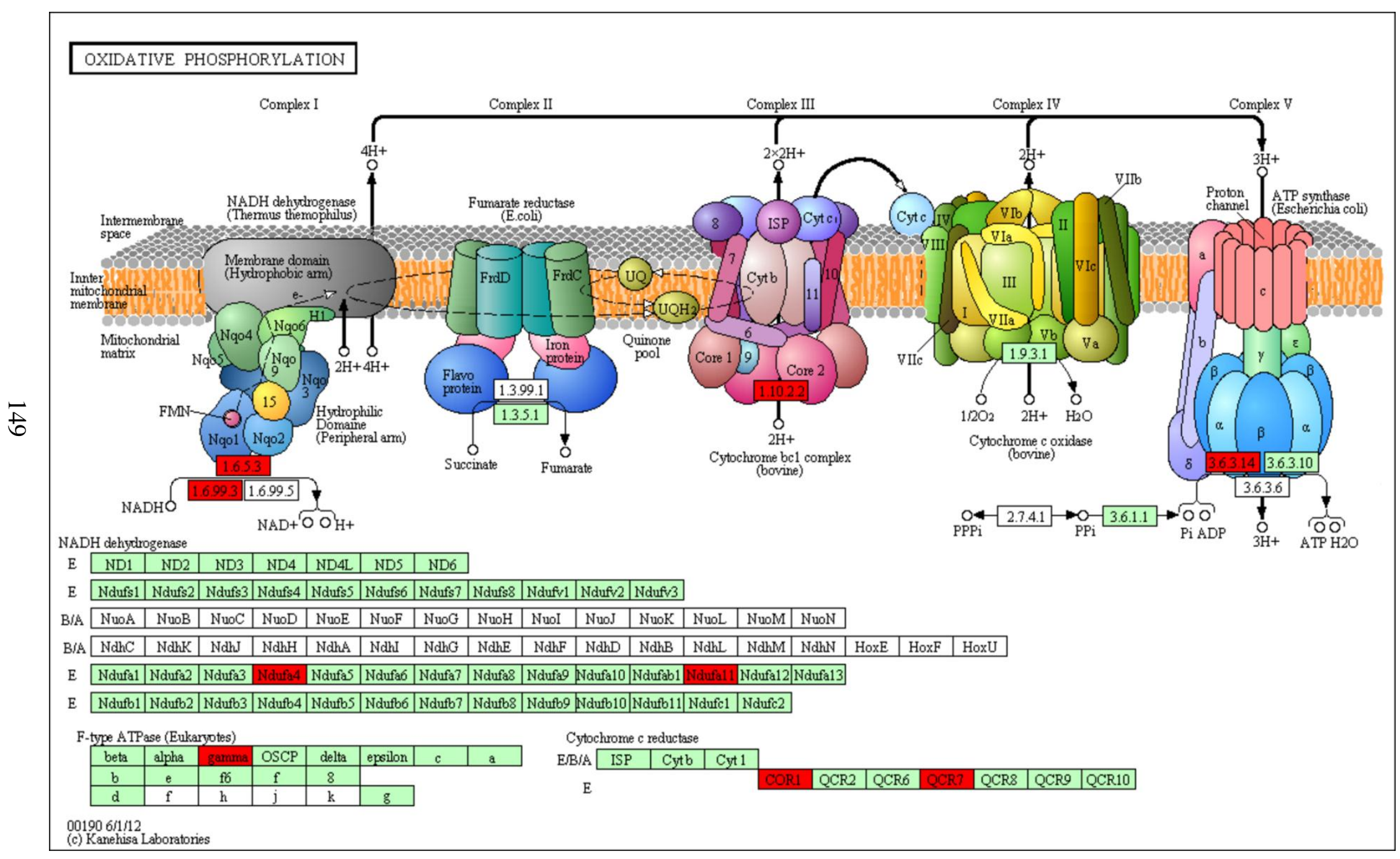


Figure 4.3. Effect of Escherichia coli lipopolysaccharide (LPS) on the toll-like receptor (TLR) signaling pathway in neutrophils (PMNL) regardless of dietary supplementation. Cows received $56 \mathrm{~g} /$ day of either OmniGen- $\mathrm{AF}^{\circledR}(\mathrm{n}=5)$ or sham control (soybean hulls; $\mathrm{n}=5$ ) mixed into total-mixed rations from day $46 \pm 1$ before calving until day 31 after parturition. PMNL were collected from the blood of cows on day 7 postpartum and then incubated with 0 or $50 \mu \mathrm{g} / \mathrm{mL}$ of LPS for $120 \mathrm{~min}$ to determine their global gene expression using RNA-sequencing. Genes with altered expression due to incubation with $50 \mu \mathrm{g} / \mathrm{mL}$ of LPS (relative to $0 \mu \mathrm{g} / \mathrm{mL}$ of LPS) were loaded to DAVID Bioinformatics Resources 6.7 where KEGG pathway analysis was applied to uncover enriched functional pathways. Objects highlighted in orange represent up-regulated gene products. Green boxes correspond to gene products specific for the Bos Taurus genome that were unaltered by treatments. Significance of gene-pathway enrichment conducted with a modified Fisher's exact test (EASE score, $P=0.038$ ). 


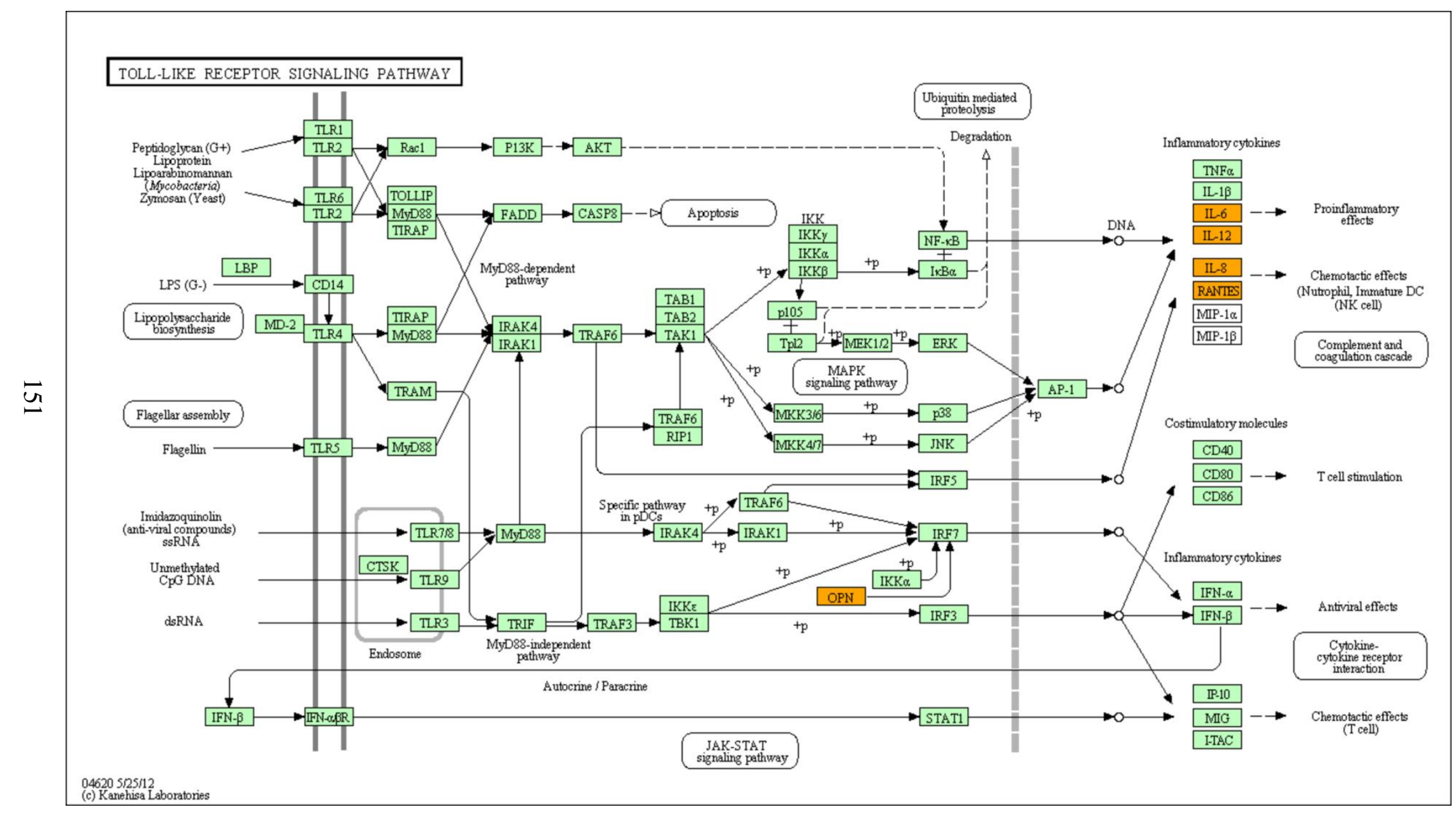


Figure 4.4. Effect of Escherichia coli lipopolysaccharide (LPS) on the hematopoietic cell lineage signaling pathway in neutrophils (PMNL) regardless of dietary supplementation. Cows received $56 \mathrm{~g} /$ day of either OmniGen- $\mathrm{AF}^{\circledR}(\mathrm{n}=5)$ or sham control (soybean hulls; $n=5$ ) mixed into total-mixed rations from day $46 \pm 1$ before calving until day 31 after parturition. PMNL were collected from the blood of cows on day 7 postpartum and then incubated with 0 or $50 \mu \mathrm{g} / \mathrm{mL}$ of LPS for $120 \mathrm{~min}$ to determine their global gene expression using RNA-sequencing. Genes with altered expression due to incubation with $50 \mu \mathrm{g} / \mathrm{mL}$ of LPS (relative to $0 \mu \mathrm{g} / \mathrm{mL}$ of LPS) were loaded to DAVID Bioinformatics Resources 6.7 where KEGG pathway analysis was applied to uncover enriched functional pathways. Objects highlighted in orange represent up-regulated gene products. Green boxes correspond to gene products specific for the Bos Taurus genome that were unaltered by treatments. Significance of gene-pathway enrichment conducted with a modified Fisher's exact test (EASE score, $P=0.089$ ). 


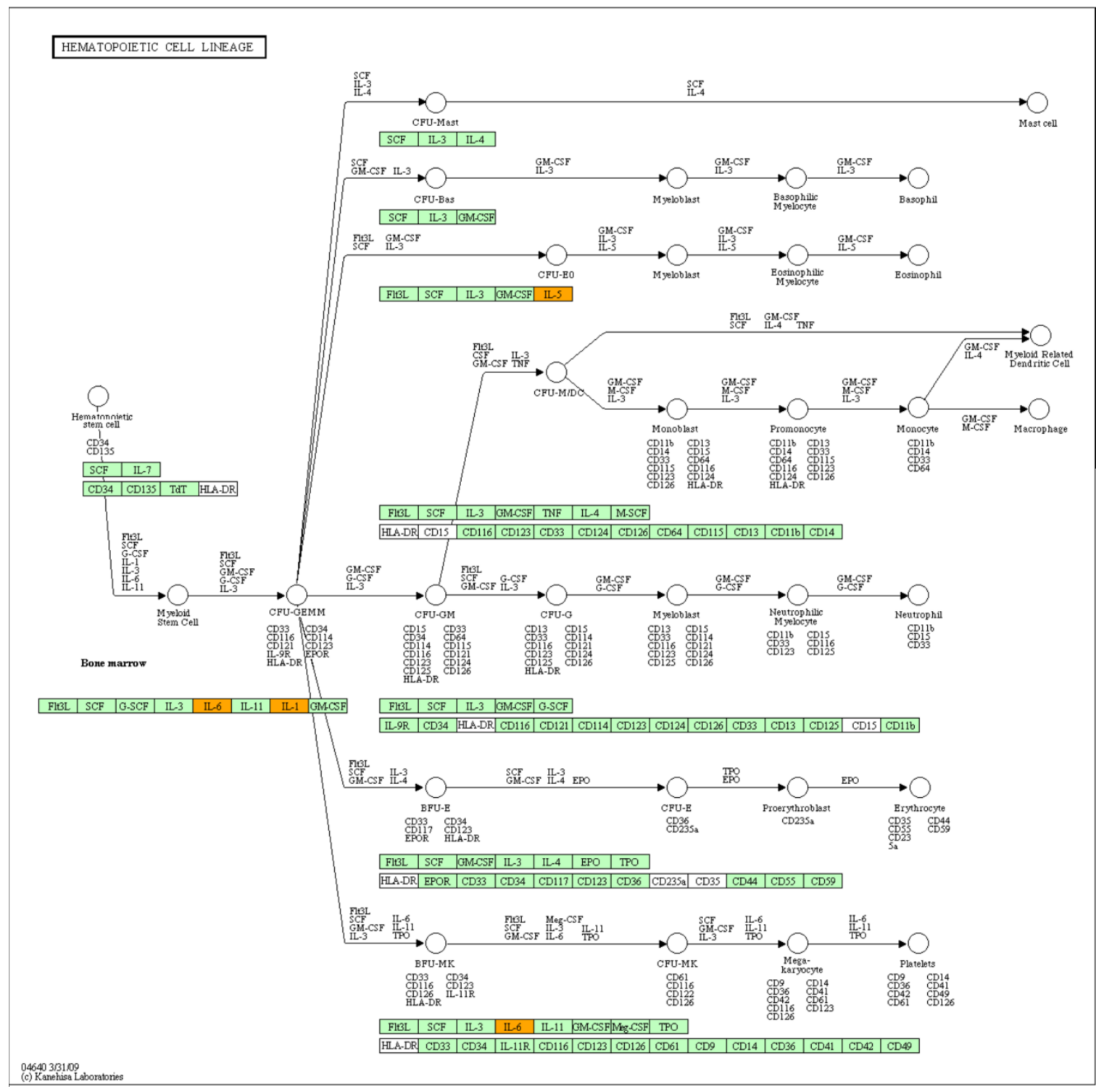


Enriched GO terms related to biological processes and molecular functions for the genes differentially regulated within each contrast are listed in Table 4.5. A total of 97 annotation terms were enriched for the genes differentially regulated across all comparisons (Table 4.5, $P<0.150$ ). The top five terms enriched in the comparison of LPS effect between PMNL harvested from OmniGen- $\mathrm{AF}^{\circledR}$ - and non-supplemented cows included RNA binding, oxidation reduction, hydrolase activity, acting on carbon-nitrogen (but not peptide) bonds, in linear amides, electron transport chain, and generation of precursor metabolites and energy (Table 4.5A, $P<0.150$ ). In the contrast between OmniGen- $\mathrm{AF}^{\circledR}$ - and non-supplemented main effects (independent of LPS incubation), the top five enriched terms were cellular macromolecular complex assembly, cellular macromolecular complex subunit organization, generation of precursor metabolites and energy, oxidation reduction, and electron transport chain (Table 4.5B, $P<0.150$ ). Finally, the top five GO terms for the main effect of LPS, regardless of dietary supplementation included intracellular signaling cascade, defense response, response to wounding, regulation of cell proliferation, and immune response. (Table 4.5B, $P<0.150$ ) 
Table 4.5. Enriched annotation terms for genes differentially regulated due to dietary supplementation with $56 \mathrm{~g} /$ day of either OmniGen-AF ${ }^{\circledR}(n=5)$ or sham control $(n=5)$ in neutrophils (PMNL) treated with 0 or $50 \mu \mathrm{g} / \mathrm{mL}$ of Escherichia coli lipopolysaccharide (LPS)

\begin{tabular}{llll}
\hline Term & Class $^{2} \uparrow$ or $\downarrow$ Genes & P value $^{3}$ \\
\hline
\end{tabular}

A. Genes differentially regulated by OmniGen-AF$F^{\circledR}$ after activation with $L P S^{1}$

RNA binding

Oxidation reduction

Hydrolase activity, acting on $\mathrm{C}-\mathrm{N}$ bonds

Electron transport chain

Generation of precursor metabolites - energy

Sphingolipid metabolic process

Membrane lipid metabolic process

$\begin{array}{llll}\text { MF } & \downarrow & 4 & 0.036 \\ \text { BP } & \downarrow & 4 & 0.096 \\ \text { MF } & \downarrow & 3 & 0.003 \\ \text { BP } & \downarrow & 3 & 0.015 \\ \text { BP } & \downarrow & 3 & 0.075 \\ \text { BP } & \downarrow & 2 & 0.055 \\ \text { BP } & \downarrow & 2 & 0.057\end{array}$

\section{B. Genes differentially regulated by OmniGen-AF ${ }^{\circledR}$ independent of $L P S^{1}$}

Cellular macromolecular complex assembly

Cellular macromolecular subunit organization

Generation of precursor metabolites -energy

Oxidation reduction

$\begin{array}{llll}\text { BP } & \uparrow & 2 & 0.100 \\ \mathrm{BP} & \uparrow & 2 & 0.110 \\ \mathrm{BP} & \downarrow & 5 & 0.001 \\ \mathrm{BP} & \downarrow & 5 & 0.018 \\ \mathrm{BP} & \downarrow & 4 & 0.001 \\ \mathrm{BP} & \downarrow & 3 & 0.007 \\ \mathrm{BP} & \downarrow & 3 & 0.059 \\ \mathrm{BP} & \downarrow & 2 & 0.010 \\ \mathrm{BP} & \downarrow & 2 & 0.028 \\ \mathrm{BP} & \downarrow & 2 & 0.034 \\ \mathrm{BP} & \downarrow & 2 & 0.042 \\ \mathrm{MF} & \downarrow & 2 & 0.049 \\ \mathrm{BP} & \downarrow & 2 & 0.052 \\ \mathrm{BP} & \downarrow & 2 & 0.054 \\ \mathrm{MF} & \downarrow & 2 & 0.076 \\ \mathrm{BP} & \downarrow & 2 & 0.094 \\ \mathrm{MF} & \downarrow & 2 & 0.095 \\ \mathrm{MF} & \downarrow & 2 & 0.100 \\ \mathrm{BP} & \downarrow & 2 & 0.130 \\ \mathrm{MF} & \downarrow & 2 & 0.140 \\ \mathrm{MF} & \downarrow & 2 & 0.140\end{array}$


Table 4.5 (continued)

Term $\quad$ Class $^{2} \uparrow$ or $\downarrow$ Genes $\quad \mathrm{P}$ value ${ }^{3}$

C. Genes differentially regulated by LPS independent of dietary supplementation ${ }^{1}$

Intracellular signaling cascade

Defense response

Response to wounding

Regulation of cell proliferation

Immune response

Cytokine activity

Inflammatory response

Carboxylic acid biosynthetic process

Organic acid biosynthetic process

Cell migration

Localization of cell

Cell motility

Cell motion

Fatty acid metabolic process

Lipid biosynthetic process

Cellular homeostasis

Growth factor activity

Acute inflammatory response

Fatty acid biosynthetic process

Blood vessel development

Skeletal system development

Vasculature development

Behavior

Acute-phase response

Unsaturated fatty acid metabolic process

Icosanoid metabolic process

Regulation of secretion

Regulation of protein-AA phosphorylation

Regulation of cellular localization

Heart development

Regulation of protein modification process

Leukocyte activation

Heme binding

Tetrapyrrole binding

Unsaturated fatty acid biosynthetic process

Icosanoid biosynthetic process

Regulation of hormone secretion

\begin{tabular}{lllr}
$\mathrm{BP}$ & $\uparrow$ & 12 & 0.010 \\
$\mathrm{BP}$ & $\uparrow$ & 10 & $<0.001$ \\
$\mathrm{BP}$ & $\uparrow$ & 9 & $<0.001$ \\
$\mathrm{BP}$ & $\uparrow$ & 9 & 0.006 \\
$\mathrm{BP}$ & $\uparrow$ & 9 & 0.008 \\
$\mathrm{MF}$ & $\uparrow$ & 9 & $<0.001$ \\
$\mathrm{BP}$ & $\uparrow$ & 8 & $<0.001$ \\
$\mathrm{BP}$ & $\uparrow$ & 7 & $<0.001$ \\
$\mathrm{BP}$ & $\uparrow$ & 7 & $<0.001$ \\
$\mathrm{BP}$ & $\uparrow$ & 7 & 0.001 \\
$\mathrm{BP}$ & $\uparrow$ & 7 & 0.001 \\
$\mathrm{BP}$ & $\uparrow$ & 7 & 0.001 \\
$\mathrm{BP}$ & $\uparrow$ & 7 & 0.003 \\
$\mathrm{BP}$ & $\uparrow$ & 6 & 0.005 \\
$\mathrm{BP}$ & $\uparrow$ & 6 & 0.026 \\
$\mathrm{BP}$ & $\uparrow$ & 6 & 0.062 \\
$\mathrm{MF}$ & $\uparrow$ & 6 & 0.003 \\
$\mathrm{BP}$ & $\uparrow$ & 5 & 0.001 \\
$\mathrm{BP}$ & $\uparrow$ & 5 & 0.002 \\
$\mathrm{BP}$ & $\uparrow$ & 5 & 0.029 \\
$\mathrm{BP}$ & $\uparrow$ & 5 & 0.031 \\
$\mathrm{BP}$ & $\uparrow$ & 5 & 0.032 \\
$\mathrm{BP}$ & $\uparrow$ & 5 & 0.084 \\
$\mathrm{BP}$ & $\uparrow$ & 4 & 0.000 \\
$\mathrm{BP}$ & $\uparrow$ & 4 & 0.004 \\
$\mathrm{BP}$ & $\uparrow$ & 4 & 0.004 \\
$\mathrm{BP}$ & $\uparrow$ & 4 & 0.034 \\
$\mathrm{BP}$ & $\uparrow$ & 4 & 0.037 \\
$\mathrm{BP}$ & $\uparrow$ & 4 & 0.063 \\
$\mathrm{BP}$ & $\uparrow$ & 4 & 0.070 \\
$\mathrm{BP}$ & $\uparrow$ & 4 & 0.070 \\
$\mathrm{BP}$ & $\uparrow$ & 4 & 0.090 \\
$\mathrm{MF}$ & $\uparrow$ & 4 & 0.089 \\
$\mathrm{MF}$ & $\uparrow$ & 4 & 0.100 \\
$\mathrm{BP}$ & $\uparrow$ & 3 & 0.018 \\
$\mathrm{BP}$ & $\uparrow$ & 3 & 0.018 \\
$\mathrm{BP}$ & $\uparrow$ & 3 & 0.023 \\
\hline & & &
\end{tabular}


Table 4.5 (continued)

\begin{tabular}{|c|c|c|c|c|}
\hline Term & Class $^{2}$ & $\uparrow$ or $\downarrow$ & Genes & $P$ value $^{3}$ \\
\hline Cytokine-mediated signaling pathway & $\mathrm{BP}$ & $\uparrow$ & 3 & 0.025 \\
\hline Cartilage development & $\mathrm{BP}$ & $\uparrow$ & 3 & 0.044 \\
\hline Response to hypoxia & $\mathrm{BP}$ & $\uparrow$ & 3 & 0.047 \\
\hline Response to oxygen levels & $\mathrm{BP}$ & $\uparrow$ & 3 & 0.047 \\
\hline Taxis & $\mathrm{BP}$ & $\uparrow$ & 3 & 0.097 \\
\hline Chemotaxis & $\mathrm{BP}$ & $\uparrow$ & 3 & 0.097 \\
\hline Ossification & $\mathrm{BP}$ & $\uparrow$ & 3 & 0.100 \\
\hline Bone development & $\mathrm{BP}$ & $\uparrow$ & 3 & 0.100 \\
\hline Chemokine activity & MF & $\uparrow$ & 3 & 0.048 \\
\hline Chemokine receptor binding & MF & $\uparrow$ & 3 & 0.048 \\
\hline Induction of positive chemotaxis & $\mathrm{BP}$ & $\uparrow$ & 2 & 0.058 \\
\hline Neutrophil chemotaxis & $\mathrm{BP}$ & $\uparrow$ & 2 & 0.067 \\
\hline Regulation of viral genome replication & $\mathrm{BP}$ & $\uparrow$ & 2 & 0.067 \\
\hline Positive regulation of positive chemotaxis & $\mathrm{BP}$ & $\uparrow$ & 2 & 0.086 \\
\hline Positive regulation of mitosis & $\mathrm{BP}$ & $\uparrow$ & 2 & 0.086 \\
\hline Regulation of positive chemotaxis & $\mathrm{BP}$ & $\uparrow$ & 2 & 0.086 \\
\hline Bone morphogenesis & $\mathrm{BP}$ & $\uparrow$ & 2 & 0.086 \\
\hline Positive regulation of nuclear division & $\mathrm{BP}$ & $\uparrow$ & 2 & 0.086 \\
\hline Regulation of viral reproduction & $\mathrm{BP}$ & $\uparrow$ & 2 & 0.086 \\
\hline Endochondral ossification & $\mathrm{BP}$ & $\uparrow$ & 2 & 0.086 \\
\hline Chondrocyte differentiation & $\mathrm{BP}$ & $\uparrow$ & 2 & 0.086 \\
\hline Endochondral bone morphogenesis & $\mathrm{BP}$ & $\uparrow$ & 2 & 0.086 \\
\hline Calcium-mediated signaling & $\mathrm{BP}$ & $\uparrow$ & 2 & 0.095 \\
\hline Regulation of smooth muscle cell proliferation & $\mathrm{BP}$ & $\uparrow$ & 2 & 0.095 \\
\hline Fibroblast growth factor receptor antagonism & MF & $\uparrow$ & 2 & 0.041 \\
\hline Fibroblast growth factor receptor binding & MF & $\uparrow$ & 2 & 0.041 \\
\hline Interleukin-1 receptor antagonist activity & MF & $\uparrow$ & 2 & 0.041 \\
\hline Receptor antagonist activity & MF & $\uparrow$ & 2 & 0.051 \\
\hline Receptor inhibitor activity & MF & $\uparrow$ & 2 & 0.061 \\
\hline Receptor regulator activity & MF & $\uparrow$ & 2 & 0.090 \\
\hline Posttranscriptional regulation & $\mathrm{BP}$ & $\downarrow$ & 3 & 0.071 \\
\hline Deacetylase activity & MF & $\downarrow$ & 2 & 0.064 \\
\hline
\end{tabular}

${ }^{\mathrm{T}}$ Cows were fed $56 \mathrm{~g} /$ day of either OmniGen- $\mathrm{AF}^{\circledR}(\mathrm{n}=5)$ or sham control (soybean hulls; $\mathrm{n}=5$ ) from day $46 \pm 1$ prepartum until day 31 postpartum. On day 7 postpartum, PMNL were harvested from blood and then incubated with 0 or $50 \mu \mathrm{g} / \mathrm{mL}$ of Escherichia coli lipopolysaccharide (LPS) for $120 \mathrm{~min}$.

${ }^{2} \mathrm{BP}=$ Biological process, $\mathrm{MF}=$ Molecular function.

${ }^{3}$ Significance of gene-term enrichment test conducted with a modified Fisher's exact test (EASE score). 


\section{DISCUSSION}

To our knowledge, this is the first study designed to investigate changes in global gene expression in PMNL using RNA-seq technology. Although PMNL are commonly thought to have limited transcriptional capabilities, human PMNL transcribe a wide repertoire of genes involved in immune response (Newburger et al., 2000). In the current study with bovine PMNL, we generated an average of 28.4 million 50-base pair reads per cDNA library that passed the quality filters. Even though a very high number of these fragments mapped to the bovine genome ( $88.4 \%)$, the average percent coverage was only 9.7\% (Table 4.4). This second finding is not surprising because the bovine genome, similarly to most mammalian genomes, has a high percentage of non-coding and repetitive DNA sequences (Elsik et al., 2009). Nevertheless, the PMNL in our experiments expressed approximately 16,400 genes at a level that allowed differential expression analysis (data not shown). Assuming the bovine genome contains a minimum of 22,000 protein-coding genes (Elsik et al., 2009), our study indicates that PMNL express roughly $75 \%$ of the total protein-coding genes in the bovine genome. Although bovine PMNL are often considered as only as effector cells with a short half-life, this finding highlights that bovine PMNL have a high capacity to express protein-coding genes.

In the present study, contrast analysis of the immune-activation effect by LPS (50 vs. $0 \mu \mathrm{g} / \mathrm{mL}$ ) between PMNL harvested from OmniGen-AF${ }^{\circledR}$ - and those from nonsupplemented cows revealed 7 and 36 genes with up- and down-regulated expression, respectively. To better appreciate their biological meaning, pathway and GO enrichment analysis was conducted using these differentially expressed genes. Within this 
comparison, the lysosome pathway was detected enriched by down-regulated genes encoding for cathepsin, glucosidase beta acid (GBA), and ATP-binding cassette subfamily B member 9 (ABCB9). In agreement with the down-regulation of the lysosome pathway, GO analysis indicated that the molecular function hydrolase activity (acting on carbon-nitrogen bonds) was reduced due to OmniGen-AF ${ }^{\circledR}$ supplementation in LPSactivated PMNL. Other GO terms enriched by down-regulated genes within this contrast included electron transport chain, oxidative reduction, and generation of precursor metabolites and energy. The biological relevance of a reduced activity in the lysosome pathway is difficult to infer. Lysosomes are involved in the inactivation, destruction, and recycling of endocytic and phagocytic particles including pathogenic organisms (Eskelinen et al., 2003). Whether the potential down-regulation of lysosome function by dietary supplementation with OmniGen- $\mathrm{AF}^{\circledR}$ has implications for the immune capacity of PMNL is unclear. However, results from a complementary study conducted by our group suggested that OmniGen- $\mathrm{AF}^{\circledR}$ supplementation does not alter the antimicrobial function of PMNL harvested from dairy cows throughout the periparturient period (Revelo et al., dissertation chapter three). Furthermore, other researchers have reported that OmniGen$\mathrm{AF}^{\circledR}$ may improve immune function by restoring the protein level of L-selectin in PMNL from dexamethasone-immunosuppressed sheep (Wang et al., 2007).

Relative to non-supplemented controls, feeding Omingen-AF ${ }^{\circledR}$ to dairy cows resulted in the up-regulation of 12 and down-regulation of 41 genes (regardless of the LPS activation effect). Using microarrays, Wang et al. (2009b) reported that OmniGen$\mathrm{AF}^{\circledR}$ supplementation to dairy cows during the dry period caused greater expression of 12 genes and decreased expression of six genes involved in PMNL apoptosis, cell-to-cell 
communication, and intracellular signaling. In the current study a higher number of differentially regulated genes were detected using RNA-sequencing, however, no common genes were found compared to those reported by Wang et al. (2009). This discrepancy may be explained by the techniques used to estimate gene expression or by the timing of PMNL collection. These investigators purified PMNL from blood collected 15 hours after parturition whereas we harvested PMNL on day 7 postpartum. Furthermore, our functional annotation analysis revealed that the oxidative phosphorylation pathway was enriched with genes down-regulated by OmniGen-AF ${ }^{\circledR}$ supplementation. Consistently, several enriched GO terms complementary to this pathway were identified including oxidative phosphorylation, oxidation reduction, electron transport chain, negative regulation of biosynthetic process, mitochondrial electron transport, ubiquinol to cytochrome $\mathrm{c}$, mitochondrial ATP synthesis coupled electron transport, ATP synthesis coupled electron transport, respiratory electron transport chain, cation-transporting ATPase activity, and cellular respiration. Unlike other cells, leukocytes rely on glycolysis rather than on oxidative phosphorylation as their main source of energy under both low (hypoxic) and normal $\mathrm{O}_{2}$ conditions (Borregaard and Herlin, 1982). Hypoxia, however, is not optimal for immune cells and often impairs their effector functions including their ROS production, a process that requires considerable amounts of cellular $\mathrm{O}_{2}$ (Sitkovsky and Lukashev, 2005). Therefore, one can speculate that PMNL with decreased oxidative phosphorylation may be more efficient in distributing $\mathrm{O}_{2}$ towards effector functions with a high demand for $\mathrm{O}_{2}$ such as ROS generation. This increased efficiency may be particularly important during hypoxic conditions such as during an inflammatory response. 
Another interesting finding was that Omingen- $\mathrm{AF}^{\circledR}$ supplementation (independent of LPS incubation) increased the expression of tumor necrosis factor receptor superfamily, member 17 (TNFRSF) by approximately 130\%. TNFRSF encodes for a protein member of the TNF-receptor superfamily, which transduces signals that lead to immune activation upon binding of its ligand (Armitage, 1994). This up-regulated expression of TNFRSF suggests that the dietary supplement Omingen- $\mathrm{AF}^{\circledR}$ may increase PMNL responsiveness to inflammatory signals. Our laboratory has previously reported that PMNL from early lactation cows have reduced LPS-dependent expression of TNF and other inflammatory mediators (Revelo and Waldron, 2012). This hyporesponsiveness of the immune system to antigen may contribute to periparturient immunosuppression in dairy cows (Mallard et al., 1998). Therefore, improvement of PMNL response to stimuli may be critical during this period when the dairy cow is more susceptible to infections.

The third contrast in our analysis revealed the effect of LPS incubation (50 vs. 0 $\mu \mathrm{g} / \mathrm{mL}$ ) on PMNL gene expression regardless of dietary treatment. For this comparison, 211 and 122 up- and down-regulated transcripts were detected. The pathways enriched by differentially regulated LPS-responsive genes included the TLR and hematopoietic cell lineage signaling pathways. Genes with up-regulated expression due to LPS that belong to the TLR signaling pathway encode for IL-6, IL-8, IL-12, chemokine (C-C motif) ligand 5 (RANTES), and secreted phosphoprotein 1 (SSP1). Consistently, GO analysis showed several terms related to immune activation including defense response, immune response, cytokine-mediated signaling pathway, cytokine activity, inflammatory response, cell migration, cell motility, cell motion, chemotaxis, chemokine activity, 
chemokine receptor binding, and neutrophil chemotaxis. The TLRs are a subtype of PRRs used by immune cells to recognize and bind highly conserved molecular motifs known as pathogen-associated molecular patterns or PAMPs (Werling et al., 2006). In the infected mammary gland, recognition of PAMPs triggers the inflammatory response by stimulating the synthesis and release of pro-inflammatory cytokines into the surrounding environment (Bannerman, 2009). Several groups including our laboratory have previously reported that LPS enhances the gene expression of cytokines involved the inflammatory response such as IL-8 (Worku and Morris, 2009; Revelo and Waldron, 2012). Interestingly, Worku and Morris (2009) reported that E. coli LPS increases the expression of TLR- 4 on the cell surface but does not alter its mRNA contents in bovine PMNL. As discussed by these authors, LPS may induce the recruitment of TLR-4 to the cell membrane without changes in the expression of the gene. In agreement, our results indicate LPS may stimulate the PMNL via the TLR pathway without an increase in the mRNA contents of TLR-4. Although mammary macrophages and epithelial cells may be the main cell types involved in the initiation of the inflammatory response, together these findings confirm that PMNL play an important role in the synthesis of pro-inflammatory cytokines. In addition, the up-regulation of the hematopoietic cell lineage pathway suggests that LPS promote the development of common myeloid progenitors into fully differentiated granulocytes. In support of the hematopoietic functions inherent to these genes up-regulated by LPS, we found several annotation terms related to this pathway such as regulation of cell proliferation, growth factor activity, and positive regulation of nuclear division. 


\section{CONCLUSIONS}

Feeding the B-vitamin and fungal dietary supplement OmniGen- $\mathrm{AF}^{\circledR}$ to dairy cows during the periparturient period resulted in the up- and down-regulation of seven and 36 genes in PMNL upon activation with LPS. Further functional annotation analysis indicated that OmniGen- $\mathrm{AF}^{\circledR}$ supplementation down-regulated genes that enriched the lysosome pathway. Although the biological relevance of this finding at the gene expression level is difficult to infer, reduced lysosome activity may cause inefficient inactivation and destruction of phagocytic products. Independently of LPS activation, OmniGen- $\mathrm{AF}^{\circledR}$ supplementation altered the expression of 53 transcripts, (main effect of dietary supplementation). Pathway analysis revealed that down-regulated genes due to OmniGen- $\mathrm{AF}^{\circledR}$ supplementation enriched the oxidative phosphorylation pathway. It is possible that PMNL with lower oxidative phosphorylation activity may be more efficient in distributing $\mathrm{O}_{2}$ towards effector functions with a high demand for $\mathrm{O}_{2}$ such as the

production of ROS. In response to OmniGen- $\mathrm{AF}^{\circledR}$ supplementation, we also detected a strong increase in the mRNA expression of TNFRSF, a member of the TNF-receptor family. This later finding suggests that the dietary supplement may improve PMNL responsiveness to immune activation. Finally, PMNL incubation with $50 \mu \mathrm{g} / \mathrm{mL}$ of LPS altered the expression of 333 genes, independent of dietary treatment. This LPS activation in PMNL resulted in the enrichment of the TLR signaling and hematopoietic cell lineage pathways revealing potential molecular mechanisms by which LPS stimulates the immune response in bovine PMNL. 
Table 4.6. Supplemental. Differential expression of genes in neutrophils (PMNL) from cows fed either $56 \mathrm{~g} / \mathrm{day}$ of either the supplement OmniGen- $\mathrm{AF}^{\circledR}(\mathrm{n}=5$ ) or sham control (soybean hulls; $\mathrm{n}=5$ ) upon a 120 -min incubation with 0 or $50 \mu \mathrm{g} / \mathrm{mL}$ of Escherichia coli lipopolysaccharide (LPS)

\begin{tabular}{|c|c|c|}
\hline Gene ID & Full name & $\begin{array}{l}\text { Percent } \\
\text { change }^{1}\end{array}$ P-Value \\
\hline
\end{tabular}

A. Genes differentially regulated by OmniGen-AF ${ }^{\circledR}$ supplementation relative to the control diet (upon activation with LPS)

\begin{tabular}{|c|c|c|c|c|}
\hline 100298870 & LOC10029887 & Uncharacterized LOC100298870 & $68.2 \uparrow$ & 0.002 \\
\hline$\underline{539113}$ & CHRNA6 & Cholinergic receptor, nicotinic, alpha 6 & $36.6 \uparrow$ & 0.008 \\
\hline$\underline{100337120}$ & LOC10033712 & T-cell activation Rho gtpase-activating protein-like & $34.5 \uparrow$ & 0.030 \\
\hline$\underline{528156}$ & NR2E1 & Nuclear receptor subfamily 2 , group E, member 1 & $27.5 \uparrow$ & 0.036 \\
\hline$\underline{281683}$ & LECT1 & Leukocyte cell derived chemotaxin 1 & $22.9 \uparrow$ & 0.002 \\
\hline$\underline{280801}$ & GAT & Glycine-N-acyltransferase-like & $21.8 \uparrow$ & 0.005 \\
\hline$\underline{282006}$ & PROS1 & Protein S (alpha) & $13.6 \uparrow$ & 0.035 \\
\hline$\underline{517400}$ & SNX30 & Sorting nexin family member 30 & $13.8 \downarrow$ & 0.034 \\
\hline$\underline{613665}$ & LEPROT & Leptin receptor overlapping transcript & $15.6 \downarrow$ & 0.046 \\
\hline$\overline{534704}$ & TPD52L2 & Tumor protein D52-like 2 & $16.8 \downarrow$ & 0.031 \\
\hline$\underline{511610}$ & SURF6 & Surfeit 6 & $17.2 \downarrow$ & 0.040 \\
\hline$\underline{517446}$ & AP2M1 & Adaptor-related protein complex $2, \mathrm{mu} 1$ subunit & $17.9 \downarrow$ & 0.045 \\
\hline$\underline{541149}$ & LOC541149 & Transmembrane protein $185 \mathrm{~A}$-like & $19 \downarrow$ & 0.042 \\
\hline$\underline{281998}$ & PRDX3 & Peroxiredoxin 3 & $19.9 \downarrow$ & 0.032 \\
\hline$\underline{539223}$ & CERS2 & Ceramide synthase 2 & $19.9 \downarrow$ & 0.047 \\
\hline$\underline{100125289}$ & C9H6orf120 & Chromosome 9 open reading frame, human c6orf 120 & $21.4 \downarrow$ & 0.027 \\
\hline$\underline{511902}$ & $\mathrm{C} 25 \mathrm{H} 7$ orf43 & Chromosome 25 open reading frame, human c7orf43 & $21.8 \downarrow$ & 0.023 \\
\hline$\underline{515307}$ & RBM7 & RNA binding motif protein 7 & $22 \downarrow$ & 0.011 \\
\hline$\underline{537087}$ & GBA & Glucosidase, beta, acid & $24 \downarrow$ & 0.017 \\
\hline$\underline{327690}$ & NDUFB1 & NADH dehydrogenase (ubiquinone) 1 beta subcomplex, 1, 7kda & $24.8 \downarrow$ & 0.043 \\
\hline$\underline{767986}$ & C5H12orf29 & Chromosome 5 open reading frame, human c12orf29 & $25.1 \downarrow$ & 0.037 \\
\hline
\end{tabular}


Table 4.6 Supplemental (continued)

\begin{tabular}{|c|c|c|c|c|}
\hline Gene ID & Symbol & Full name & $\begin{array}{l}\text { Percent } \\
\text { change }\end{array}$ & P-Value \\
\hline 516214 & RENBP & Renin binding protein & $25.7 \downarrow$ & 0.029 \\
\hline$\overline{327711}$ & CTSS & Cathepsin S & $26 \downarrow$ & 0.018 \\
\hline 616871 & UQCRB & Ubiquinol-cytochrome c reductase binding protein & $26.4 \downarrow$ & 0.006 \\
\hline$\overline{533351}$ & C13H20orf4 & Chromosome 13 open reading frame, human c20orf4 & $26.5 \downarrow$ & 0.015 \\
\hline$\overline{509386}$ & TSPAN17 & Tetraspanin 17 & $26.6 \downarrow$ & 0.015 \\
\hline$\underline{529923}$ & ABCB9 & ATP-binding cassette, sub-family B (MDR/TAP), member 9 & $27 \downarrow$ & 0.003 \\
\hline$\underline{507084}$ & THG1L & Trna-histidine guanylyltransferase 1-like (S. Cerevisiae) & $27.1 \downarrow$ & 0.028 \\
\hline$\underline{100335838}$ & LOC10033583 & Polymerase (RNA) I polypeptide D, 16kda pseudogene & $27.8 \downarrow$ & 0.012 \\
\hline 513962 & LOC513962 & Tetraspanin 3 pseudogene & $28.5 \downarrow$ & 0.007 \\
\hline$\overline{519920}$ & EIF4E2 & Eukaryotic translation initiation factor $4 \mathrm{E}$ family member 2 & $31.1 \downarrow$ & 0.020 \\
\hline$\overline{515434}$ & ACRBP & Acrosin binding protein & $31.2 \downarrow$ & 0.047 \\
\hline$\overline{509943}$ & TSN & Translin & $32 \downarrow$ & 0.026 \\
\hline$\overline{780968}$ & LOC780968 & Uncharacterized LOC780968 & $36.2 \downarrow$ & 0.003 \\
\hline$\overline{532575}$ & PGLYRP3 & Peptidoglycan recognition protein 3 & $40.5 \downarrow$ & 0.001 \\
\hline$\underline{326346}$ & NDUFA11 & NADH dehydrogenase (ubiquinone) 1 alpha subcomplex, $11,14.7 \mathrm{kda}$ & $42.7 \downarrow$ & 0.006 \\
\hline$\underline{614583}$ & SERTAD4 & SERTA domain containing 4 & $43.8 \downarrow$ & 0.020 \\
\hline$\underline{281420}$ & PPID & Peptidylprolyl isomerase D & $45.5 \downarrow$ & 0.022 \\
\hline$\underline{539080}$ & IRGC & Immunity-related gtpase family, cinema & $51.3 \downarrow$ & 0.001 \\
\hline$\overline{504545}$ & PPAP2C & Phosphatidic acid phosphatase type $2 \mathrm{C}$ & $53.2 \downarrow$ & 0.024 \\
\hline$\underline{514090}$ & BAK1 & BCL2-antagonist/killer 1 & $55.5 \downarrow$ & 0.030 \\
\hline 768058 & ACY1 & Aminoacylase 1 & $71.9 \downarrow$ & 0.000 \\
\hline 282638 & $\mathrm{P} 2 \mathrm{RY} 2$ & Purinergic receptor $\mathrm{P} 2 \mathrm{Y}, \mathrm{G}$-protein coupled, 2 & $74 \downarrow$ & 0.000 \\
\hline
\end{tabular}

B. Genes differentially regulated by OmniGen-A $F^{\circledR}$ supplementation relative to the control diet (independent of LPS activation)

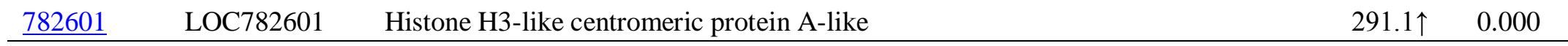


Table 4.6 Supplemental (continued)

\begin{tabular}{|c|c|c|c|c|}
\hline Gene ID & Symbol & Full name & $\begin{array}{l}\text { Percent } \\
\text { change }^{1}\end{array}$ & P-Value \\
\hline 788634 & LOC788634 & Patr class I histocompatibility antigen, A-126 alpha chain-like & $211.5 \uparrow$ & 0.000 \\
\hline$\overline{525795}$ & AGRN & Agrin & $152.2 \uparrow$ & 0.000 \\
\hline$\overline{784268}$ & TNFRSF17 & Tumor necrosis factor receptor superfamily, member 17 & $129.7 \uparrow$ & 0.009 \\
\hline$\overline{768075}$ & RCL1 & RNA terminal phosphate cyclase-like 1 & $116.4 \uparrow$ & 0.000 \\
\hline$\underline{100298822}$ & LOC10029882 & Uncharacterized LOC100298822 & $113.6 \uparrow$ & 0.000 \\
\hline$\underline{507917}$ & BOLA & MHC class I heavy chain & $106.5 \uparrow$ & 0.000 \\
\hline$\underline{613549}$ & VGLL1 & Vestigial like 1 (Drosophila) & $77.7 \uparrow$ & 0.011 \\
\hline$\underline{287019}$ & PFKFB2 & 6-phosphofructo-2-kinase/fructose-2,6-biphosphatase 2 & $53.1 \uparrow$ & 0.025 \\
\hline$\underline{540283}$ & CPOX & Coproporphyrinogen oxidase & $51.7 \uparrow$ & 0.025 \\
\hline$\underline{100337165}$ & LOC10033716 & T-cell activation Rho gtpase-activating protein-like & $40.9 \uparrow$ & 0.007 \\
\hline$\underline{100141141}$ & ZNF132 & Zinc finger protein 132 & $20.6 \uparrow$ & 0.036 \\
\hline$\underline{509613}$ & EIF4EBP1 & Eukaryotic translation initiation factor $4 \mathrm{E}$ binding protein 1 & $70 \downarrow$ & 0.001 \\
\hline$\overline{506309}$ & C8H9orf72 & Chromosome 8 open reading frame, human c9orf72 & $61.5 \downarrow$ & 0.001 \\
\hline$\underline{534904}$ & GALNT11 & UDP-N-acetyl-alpha-D-galactosamine:polypeptide N- & $51.3 \downarrow$ & 0.000 \\
\hline$\underline{513572}$ & ERAP2 & Endoplasmic reticulum aminopeptidase 2 & $47.3 \downarrow$ & 0.000 \\
\hline$\underline{515828}$ & LOC515828 & Uncharacterized LOC515828 & $46.8 \downarrow$ & 0.000 \\
\hline$\overline{514460}$ & PPP2R4 & Protein phosphatase $2 \mathrm{~A}$ activator, regulatory subunit 4 & $44.7 \downarrow$ & 0.003 \\
\hline$\underline{613512}$ & LOC613512 & Hypoxanthine phosphoribosyltransferase 1-like pseudogene & $44.4 \downarrow$ & 0.036 \\
\hline$\underline{514939}$ & PGD & Phosphogluconate dehydrogenase & $43.4 \downarrow$ & 0.001 \\
\hline$\underline{615289}$ & ABHD14B & Abhydrolase domain containing 14B & $43 \downarrow$ & 0.001 \\
\hline$\underline{617650}$ & GCHFR & GTP cyclohydrolase I feedback regulator & $42.8 \downarrow$ & 0.025 \\
\hline$\underline{100300734}$ & FAM189B & Family with sequence similarity 189 , member B & $42.2 \downarrow$ & 0.019 \\
\hline$\underline{613932}$ & PLD3 & Phospholipase D family, member 3 & $40.9 \downarrow$ & 0.006 \\
\hline$\underline{326346}$ & NDUFA11 & NADH dehydrogenase (ubiquinone) 1 alpha subcomplex, $11,14.7 \mathrm{kda}$ & $39.3 \downarrow$ & 0.030 \\
\hline$\underline{509019}$ & ZBTB7B & Zinc finger and BTB domain containing 7B & $38.5 \downarrow$ & 0.006 \\
\hline$\underline{616476}$ & HNRNPUL2 & Heterogeneous nuclear ribonucleoprotein U-like 2 & $37.7 \downarrow$ & 0.002 \\
\hline
\end{tabular}


Table 4.6 Supplemental (continued)

\begin{tabular}{|c|c|c|c|c|}
\hline Gene ID & Symbol & Full name & $\begin{array}{l}\text { Percent } \\
\text { change }^{1}\end{array}$ & P-Value \\
\hline$\underline{616015}$ & C11H9orf16 & Chromosome 11 open reading frame, human c9orf16 & $37.6 \downarrow$ & 0.036 \\
\hline$\underline{505738}$ & MCOLN1 & Mucolipin 1 & $37.4 \downarrow$ & 0.027 \\
\hline$\underline{784324}$ & LOC784324 & Chromosome 7 open reading frame, human c19orf50 pseudogene & $35.7 \downarrow$ & 0.012 \\
\hline$\underline{504586}$ & ASNA1 & Arsa arsenite transporter, ATP-binding, homolog 1 (bacterial) & $35.2 \downarrow$ & 0.016 \\
\hline$\underline{511816}$ & SNAPIN & SNAP-associated protein & $34.9 \downarrow$ & 0.031 \\
\hline$\underline{504595}$ & DENND2D & DENN/MADD domain containing 2D & $34.1 \downarrow$ & 0.001 \\
\hline$\underline{327704}$ & NDUFA4 & NADH dehydrogenase (ubiquinone) 1 alpha subcomplex, 4, 9kda & $32.8 \downarrow$ & 0.014 \\
\hline$\underline{616871}$ & UQCRB & Ubiquinol-cytochrome c reductase binding protein & $31.7 \downarrow$ & 0.007 \\
\hline$\underline{767822}$ & PNPLA6 & Patatin-like phospholipase domain containing 6 & $30.9 \downarrow$ & 0.006 \\
\hline$\underline{282393}$ & UQCRC1 & Ubiquinol-cytochrome $\mathrm{c}$ reductase core protein I & $30.6 \downarrow$ & 0.004 \\
\hline$\underline{100335838}$ & LOC10033583 & Polymerase (RNA) I polypeptide D, 16kda pseudogene & $29.9 \downarrow$ & 0.026 \\
\hline$\underline{282024}$ & TWF2 & Twinfilin, actin-binding protein, homolog 2 (Drosophila) & $29 \downarrow$ & 0.017 \\
\hline$\underline{539223}$ & LASS2 & Ceramide synthase 2 & $28.1 \downarrow$ & 0.025 \\
\hline$\underline{509725}$ & DIMT1L & DIM1 dimethyladenosine transferase 1 homolog (S. Cerevisiae) & $26.6 \downarrow$ & 0.023 \\
\hline$\underline{541149}$ & LOC541149 & Transmembrane protein $185 \mathrm{~A}$-like & $26.4 \downarrow$ & 0.020 \\
\hline$\underline{525164}$ & TRIAP1 & TP53 regulated inhibitor of apoptosis 1 & $26.3 \downarrow$ & 0.046 \\
\hline$\underline{513148}$ & DHX35 & DEAH (Asp-Glu-Ala-His) box polypeptide 35 & $26 \downarrow$ & 0.013 \\
\hline$\underline{505570}$ & C13H20orf11 & Chromosome 13 open reading frame, human c20orf11 & $25.8 \downarrow$ & 0.044 \\
\hline$\underline{100138035}$ & TMCO6 & Transmembrane and coiled-coil domains 6 & $25.1 \downarrow$ & 0.014 \\
\hline 767938 & GBAS & Glioblastoma amplified sequence & $24.9 \downarrow$ & 0.008 \\
\hline$\underline{537087}$ & GBA & Glucosidase, beta, acid & $24.6 \downarrow$ & 0.047 \\
\hline$\underline{534704}$ & TPD52L2 & Tumor protein D52-like 2 & $23.4 \downarrow$ & 0.017 \\
\hline$\underline{517097}$ & CTCF & CCCTC-binding factor (zinc finger protein) & $21.5 \downarrow$ & 0.029 \\
\hline$\underline{519624}$ & STX16 & Syntaxin 16 & $21.1 \downarrow$ & 0.036 \\
\hline$\underline{534287}$ & FBXO3 & F-box protein 3 & $21 \downarrow$ & 0.015 \\
\hline$\underline{327668}$ & ATP5C1 & ATP synthase, H+ transporting, mitochondrial F1 complex, gamma polypeptide 1 & $20.5 \downarrow$ & 0.022 \\
\hline
\end{tabular}


Table 4.6 Supplemental (continued)

\begin{tabular}{lll}
\hline Gene ID $\quad$ Symbol & Full name & $\begin{array}{c}\text { Percent } \\
\text { change }^{1}\end{array}$ P-Value \\
\hline
\end{tabular}

C. Genes differentially regulated by incubation with 50 relative to $0 \mu \mathrm{g} / \mathrm{mL}$ of LPS (independent of dietary supplementation)

\begin{tabular}{|c|c|c|c|c|}
\hline$\underline{281499}$ & SPP1 & Secreted phosphoprotein 1 & $500.1 \uparrow$ & 0.000 \\
\hline$\underline{280826}$ & IL6 & Interleukin 6 (interferon, beta 2) & $481.1 \uparrow$ & 0.002 \\
\hline$\underline{513152}$ & RHOC & Ras homolog gene family, member $\mathrm{C}$ & $436.1 \uparrow$ & 0.001 \\
\hline$\underline{616035}$ & SAA1 & Serum amyloid A1 & $383.7 \uparrow$ & 0.000 \\
\hline$\underline{281474}$ & SAA3 & Serum amyloid A 3 & $380.9 \uparrow$ & 0.001 \\
\hline$\underline{618238}$ & M-SAA3.2 & Mammary serum amyloid A3.2 & $309.8 \uparrow$ & 0.003 \\
\hline$\underline{514076}$ & CFB & Complement factor B & $304.8 \uparrow$ & 0.003 \\
\hline$\underline{515276}$ & LGALS16 & Lectin, galactoside-binding, soluble, 16 & $287 \uparrow$ & 0.002 \\
\hline$\underline{506412}$ & SAA2 & Serum amyloid A2 & $272 \uparrow$ & 0.002 \\
\hline 282023 & PTGS2 & Prostaglandin-endoperoxide synthase 2 (prostaglandin G/H synthase and & $255.8 \uparrow$ & 0.000 \\
\hline$\underline{100138376}$ & LOC10013837 & Uncharacterized LOC100138376 & $246.5 \uparrow$ & 0.000 \\
\hline$\underline{281856}$ & IL12A & Interleukin $12 \mathrm{~A}$ (natural killer cell stimulatory factor 1 , cytotoxic lymphocyte & $234.9 \uparrow$ & 0.015 \\
\hline$\underline{281137}$ & EDN1 & Endothelin 1 & $224.6 \uparrow$ & 0.003 \\
\hline$\underline{615762}$ & IL36G & Interleukin 36, gamma & $218.3 \uparrow$ & 0.006 \\
\hline$\underline{539640}$ & RCAN1 & Regulator of calcineurin 1 & $184.1 \uparrow$ & 0.005 \\
\hline$\underline{360007}$ & TFPI2 & Tissue factor pathway inhibitor 2 & $165.2 \uparrow$ & 0.002 \\
\hline 282876 & NOS2 & Nitric oxide synthase 2 , inducible & $163.8 \uparrow$ & 0.001 \\
\hline$\underline{100299023}$ & LOC10029902 & Lectin, galactoside-binding, soluble, 14-like & $162.5 \uparrow$ & 0.013 \\
\hline$\underline{538516}$ & PDLIM3 & PDZ and LIM domain 3 & $112.8 \uparrow$ & 0.002 \\
\hline$\underline{281214}$ & CXCL2 & Chemokine (C-X-C motif) ligand 2 & $110.5 \uparrow$ & 0.001 \\
\hline$\underline{281250}$ & IL1A & Interleukin 1, alpha & $96.4 \uparrow$ & 0.002 \\
\hline$\underline{100138099}$ & ZNF263 & Zinc finger protein 263 & $89.4 \uparrow$ & 0.003 \\
\hline 280825 & IL5 & Interleukin 5 (colony-stimulating factor, eosinophil) & $87.8 \uparrow$ & 0.001 \\
\hline 280828 & IL8 & Interleukin 8 & $84.3 \uparrow$ & 0.003 \\
\hline
\end{tabular}


Table 4.6 Supplemental (continued)

\begin{tabular}{|c|c|c|c|c|}
\hline Gene ID & Symbol & Full name & $\begin{array}{l}\text { Percent } \\
\text { change }^{1}\end{array}$ & P-Value \\
\hline$\underline{510619}$ & TSPAN31 & Tetraspanin 31 & $84 \uparrow$ & 0.001 \\
\hline$\overline{523429}$ & IL36A & Interleukin 36 , alpha & $84 \uparrow$ & 0.002 \\
\hline 281489 & SLAMF1 & Signaling lymphocytic activation molecule family member 1 & $83.3 \uparrow$ & 0.006 \\
\hline$\overline{613879}$ & MED30 & Mediator complex subunit 30 & $82.1 \uparrow$ & 0.000 \\
\hline$\underline{281408}$ & PLAU & Plasminogen activator, urokinase & $75.3 \uparrow$ & 0.005 \\
\hline 784268 & TNFRSF17 & Tumor necrosis factor receptor superfamily, member 17 & $74.1 \uparrow$ & 0.007 \\
\hline$\underline{539270}$ & ABT1 & Activator of basal transcription 1 & $71.8 \uparrow$ & 0.004 \\
\hline$\underline{100296900}$ & LOC10029690 & Uncharacterized LOC100296900 & $71.3 \uparrow$ & 0.001 \\
\hline$\underline{540386}$ & DDAH2 & Dimethylarginine dimethylaminohydrolase 2 & $68.5 \uparrow$ & 0.001 \\
\hline$\underline{282151}$ & BCL2A1 & BCL2-related protein A1 & $68.3 \uparrow$ & 0.002 \\
\hline$\underline{534043}$ & CD109 & CD109 molecule & $67.3 \uparrow$ & 0.001 \\
\hline$\underline{515049}$ & NECAB2 & $\mathrm{N}$-terminal EF-hand calcium binding protein 2 & $66.7 \uparrow$ & 0.002 \\
\hline$\underline{504257}$ & $\mathrm{SCOC}$ & Short coiled-coil protein & $65.7 \uparrow$ & 0.002 \\
\hline$\underline{281983}$ & PLAUR & Plasminogen activator, urokinase receptor & $64.4 \uparrow$ & 0.004 \\
\hline$\underline{616804}$ & CTSO & Cathepsin $\mathrm{O}$ & $63.5 \uparrow$ & 0.001 \\
\hline$\underline{509296}$ & GSDMB & Gasdermin B & $63.3 \uparrow$ & 0.012 \\
\hline$\underline{540641}$ & CCRN4L & CCR4 carbon catabolite repression 4-like (S. Cerevisiae) & $62.6 \uparrow$ & 0.002 \\
\hline$\underline{524106}$ & ZNF354A & Zinc finger protein $354 \mathrm{~A}$ & $61 \uparrow$ & 0.001 \\
\hline$\underline{614795}$ & FKBP1A & FK506 binding protein $1 \mathrm{~A}, 12 \mathrm{kda}$ & $60.6 \uparrow$ & 0.001 \\
\hline$\underline{538751}$ & AREGB & Amphiregulin B & $60.5 \uparrow$ & 0.006 \\
\hline$\underline{522921}$ & HBEGF & Heparin-binding EGF-like growth factor & $58.5 \uparrow$ & 0.014 \\
\hline$\underline{100337107}$ & WDR35 & WD repeat domain 35 & $58.1 \uparrow$ & 0.008 \\
\hline$\underline{100295476}$ & EREG & Epiregulin & $57.8 \uparrow$ & 0.022 \\
\hline$\underline{538657}$ & TRIM68 & Tripartite motif containing 68 & $56.8 \uparrow$ & 0.000 \\
\hline$\underline{522795}$ & KLF10 & Kruppel-like factor 10 & $56.7 \uparrow$ & 0.001 \\
\hline 520781 & TRIM66 & Tripartite motif containing 66 & $56.7 \uparrow$ & 0.010 \\
\hline
\end{tabular}


Table 4.6 Supplemental (continued)

\begin{tabular}{|c|c|c|c|c|}
\hline Gene ID & Symbol & Full name & $\begin{array}{l}\text { Percent } \\
\text { change }^{1}\end{array}$ & P-Value \\
\hline$\underline{327712}$ & CCL5 & Chemokine (C-C motif) ligand 5 & $56.4 \uparrow$ & 0.006 \\
\hline$\overline{281716}$ & CRHR1 & Corticotropin releasing hormone receptor 1 & $56.3 \uparrow$ & 0.001 \\
\hline$\overline{512375}$ & TWISTNB & TWIST neighbor & $55.3 \uparrow$ & 0.001 \\
\hline$\underline{618656}$ & LOC618656 & Protease, serine, 38 & $54.7 \uparrow$ & 0.001 \\
\hline$\underline{514745}$ & DHCR7 & 7-dehydrocholesterol reductase & $53.8 \uparrow$ & 0.002 \\
\hline 787450 & ALOX12E & Arachidonate lipoxygenase, epidermal & $53.8 \uparrow$ & 0.028 \\
\hline$\underline{450206}$ & AQP8 & Aquaporin 8 & $53.6 \uparrow$ & 0.001 \\
\hline$\underline{616423}$ & C29H11orf48 & Chromosome 29 open reading frame, human c11orf 48 & $52.3 \uparrow$ & 0.001 \\
\hline$\underline{615890}$ & GDPD1 & Glycerophosphodiester phosphodiesterase domain containing 1 & $52.3 \uparrow$ & 0.002 \\
\hline$\underline{538384}$ & PRDM1 & PR domain containing 1 , with ZNF domain & $50.2 \uparrow$ & 0.004 \\
\hline$\underline{519461}$ & SLC22A1 & Solute carrier family 22 (organic cation transporter), member 1 & $50.2 \uparrow$ & 0.017 \\
\hline$\underline{514456}$ & GPD1L & Glycerol-3-phosphate dehydrogenase 1-like & $50.1 \uparrow$ & 0.002 \\
\hline$\underline{100301381}$ & LOC10030138 & Parvalbumin alpha-like & $48.5 \uparrow$ & 0.000 \\
\hline$\underline{535166}$ & LOC535166 & Extracellular sulfatase Sulf-1-like & $48.2 \uparrow$ & 0.001 \\
\hline$\underline{282335}$ & RABGEF1 & RAB guanine nucleotide exchange factor (GEF) 1 & $47.8 \uparrow$ & 0.006 \\
\hline$\underline{536771}$ & FGF5 & Fibroblast growth factor 5 & $45.2 \uparrow$ & 0.001 \\
\hline$\underline{788561}$ & THNSL1 & Threonine synthase-like 1 (S. Cerevisiae) & $44.7 \uparrow$ & 0.016 \\
\hline$\underline{519731}$ & TYW3 & Trna-yw synthesizing protein 3 homolog (S. Cerevisiae) & $44.1 \uparrow$ & 0.001 \\
\hline$\underline{520472}$ & NAMPT & Nicotinamide phosphoribosyltransferase & $44 \uparrow$ & 0.007 \\
\hline$\underline{538673}$ & SLC1A6 & Solute carrier family 1 (high affinity aspartate/glutamate transporter), member 6 & $43.4 \uparrow$ & 0.000 \\
\hline$\underline{518088}$ & GRID2IP & Glutamate receptor, ionotropic, delta 2 (Grid2) interacting protein & $42 \uparrow$ & 0.001 \\
\hline$\overline{509355}$ & SYAP1 & Synapse associated protein 1 & $41 \uparrow$ & 0.002 \\
\hline$\underline{511776}$ & RAB9A & RAB9A, member RAS oncogene family & $40.2 \uparrow$ & 0.002 \\
\hline$\underline{537991}$ & $\mathrm{LRCH} 2$ & Leucine-rich repeats and calponin homology $(\mathrm{CH})$ domain containing 2 & $39.8 \uparrow$ & 0.005 \\
\hline$\underline{615161}$ & FRMD5 & FERM domain containing 5 & $39.5 \uparrow$ & 0.002 \\
\hline$\underline{282329}$ & PTGER2 & Prostaglandin E receptor 2 (subtype EP2), 53kda & $37.3 \uparrow$ & 0.012 \\
\hline
\end{tabular}


Table 4.6 Supplemental (continued)

\begin{tabular}{|c|c|c|c|c|}
\hline Gene ID & Symbol & Full name & $\begin{array}{l}\text { Percent } \\
\text { change }^{1}\end{array}$ & P-Value \\
\hline$\underline{540705}$ & ETNK1 & Ethanolamine kinase 1 & $36.1 \uparrow$ & 0.001 \\
\hline$\overline{540041}$ & MATN3 & Matrilin 3 & $35.7 \uparrow$ & 0.031 \\
\hline$\underline{100138434}$ & LAMA2 & Laminin, alpha 2 & $35.2 \uparrow$ & 0.004 \\
\hline$\overline{515158}$ & CCDC99 & Coiled-coil domain containing 99 & $34.5 \uparrow$ & 0.003 \\
\hline$\overline{537379}$ & RBP1 & Retinol binding protein 1 , cellular & $34.4 \uparrow$ & 0.001 \\
\hline$\underline{541187}$ & ATP8B1 & Atpase, aminophospholipid transporter, class I, type 8B, member 1 & $34.2 \uparrow$ & 0.001 \\
\hline$\underline{504677}$ & PGAP1 & Post-GPI attachment to proteins 1 & $33.9 \uparrow$ & 0.002 \\
\hline 767921 & C24H18orf32 & Chromosome 24 open reading frame, human c18orf32 & $33.6 \uparrow$ & 0.007 \\
\hline$\underline{513276}$ & RBKS & Ribokinase & $33.3 \uparrow$ & 0.005 \\
\hline$\underline{516925}$ & COX18 & COX18 cytochrome c oxidase assembly homolog (S. Cerevisiae) & $33.1 \uparrow$ & 0.024 \\
\hline$\underline{100139386}$ & 20ALPHA- & Placental and ovary 20alpha hydroxysteroid dehydrogenase protein & $32.8 \uparrow$ & 0.007 \\
\hline$\underline{535565}$ & ANAPC1 & Anaphase promoting complex subunit 1 & $32.4 \uparrow$ & 0.002 \\
\hline$\underline{100126179}$ & ZNF75A & Zinc finger protein $75 \mathrm{a}$ & $32.3 \uparrow$ & 0.006 \\
\hline$\underline{280740}$ & CA2 & Carbonic anhydrase II & $32.3 \uparrow$ & 0.012 \\
\hline$\underline{100299171}$ & GINS2 & GINS2 GINS complex subunit 2 (Psf2 homolog) [ Bos taurus ] & $31.8 \uparrow$ & 0.002 \\
\hline$\underline{512660}$ & SEMA3C & Sema domain, immunoglobulin domain (Ig), short basic domain, secreted, & $31.4 \uparrow$ & 0.000 \\
\hline$\underline{614335}$ & RAB3IL1 & RAB3A interacting protein (rabin3)-like 1 & $31.4 \uparrow$ & 0.006 \\
\hline$\underline{535714}$ & AIFM1 & Apoptosis-inducing factor, mitochondrion-associated, 1 & $31.3 \uparrow$ & 0.002 \\
\hline$\underline{531420}$ & GP2 & Glycoprotein 2 (zymogen granule membrane) & $31.2 \uparrow$ & 0.003 \\
\hline$\underline{505502}$ & FOXP2 & Forkhead box P2 & $30.9 \uparrow$ & 0.001 \\
\hline$\underline{515321}$ & $\mathrm{BDH} 2$ & 3-hydroxybutyrate dehydrogenase, type 2 & $30.4 \uparrow$ & 0.004 \\
\hline$\underline{540652}$ & GALNT8 & UDP-N-acetyl-alpha-D-galactosamine:polypeptide N- & $30.1 \uparrow$ & 0.000 \\
\hline 786270 & LOC786270 & ZAR1-like protein-like & $29.9 \uparrow$ & 0.001 \\
\hline$\underline{527289}$ & GRXCR1 & Glutaredoxin, cysteine rich 1 & $29.5 \uparrow$ & 0.002 \\
\hline$\underline{516469}$ & ENKUR & Enkurin, TRPC channel interacting protein & $29.4 \uparrow$ & 0.005 \\
\hline 513348 & NME9 & NME gene family member 9 & $29.3 \uparrow$ & 0.004 \\
\hline
\end{tabular}


Table 4.6 Supplemental (continued)

\begin{tabular}{|c|c|c|c|c|}
\hline Gene ID & Symbol & Full name & $\begin{array}{l}\text { Percent } \\
\text { change }^{1}\end{array}$ & P-Value \\
\hline Top of & SOGA1 & Suppressor of glucose, autophagy associated 1 & $29.1 \uparrow$ & 0.001 \\
\hline$\underline{526949}$ & OXSR1 & Oxidative-stress responsive 1 & $28.8 \uparrow$ & 0.025 \\
\hline$\underline{100138604}$ & PKD1L3 & Polycystic kidney disease 1 -like 3 & $28.7 \uparrow$ & 0.009 \\
\hline$\overline{504954}$ & FILIP1L & Filamin A interacting protein 1-like & $28.6 \uparrow$ & 0.001 \\
\hline 100337027 & LOC 10033702 & Glutamate receptor, ionotropic, kainate 4 -like & $28.6 \uparrow$ & 0.003 \\
\hline 282001 & PRKCA & Protein kinase $\mathrm{C}$, alpha & $28.5 \uparrow$ & 0.006 \\
\hline$\underline{509784}$ & PHLDB2 & Pleckstrin homology-like domain, family B, member 2 & $28.3 \uparrow$ & 0.000 \\
\hline$\underline{353351}$ & BMP15 & Bone morphogenetic protein 15 & $28 \uparrow$ & 0.005 \\
\hline$\underline{517589}$ & NUDT9 & Nudix (nucleoside diphosphate linked moiety X)-type motif 9 & $27.8 \uparrow$ & 0.005 \\
\hline$\underline{281875}$ & ITGAV & Integrin, alpha V (vitronectin receptor, alpha polypeptide, antigen CD51) & $27.5 \uparrow$ & 0.003 \\
\hline$\underline{327709}$ & GSTM1 & Glutathione S-transferase M1 & $26.4 \uparrow$ & 0.001 \\
\hline$\underline{540925}$ & $\mathrm{SCN} 3 \mathrm{~B}$ & Sodium channel, voltage-gated, type III, beta & $25.8 \uparrow$ & 0.000 \\
\hline$\underline{100295005}$ & LOC10029500 & Nuclear envelope pore membrane protein POM 121-like & $25.7 \uparrow$ & 0.003 \\
\hline$\underline{617927}$ & SNTB1 & Syntrophin, beta 1 (dystrophin-associated protein A1, 59kda, basic component 1 ) & $25.6 \uparrow$ & 0.001 \\
\hline$\underline{519694}$ & ARL5A & ADP-ribosylation factor-like $5 \mathrm{~A}$ & $25.6 \uparrow$ & 0.001 \\
\hline$\underline{510749}$ & PANK3 & Pantothenate kinase 3 & $25.5 \uparrow$ & 0.002 \\
\hline$\underline{287019}$ & PFKFB2 & 6-phosphofructo-2-kinase/fructose-2,6-biphosphatase 2 & $25.3 \uparrow$ & 0.027 \\
\hline$\underline{505686}$ & LAD1 & Ladinin 1 & $25.1 \uparrow$ & 0.002 \\
\hline$\underline{505226}$ & C4H7orf46 & Chromosome 4 open reading frame, human c7orf46 & $25 \uparrow$ & 0.005 \\
\hline$\underline{616821}$ & LOC616821 & Midline 2-like & $24.8 \uparrow$ & 0.005 \\
\hline$\underline{530413}$ & SAMD15 & Sterile alpha motif domain containing 15 & $24.8 \uparrow$ & 0.009 \\
\hline$\underline{785150}$ & LOC785150 & Aquarius-like & $24.5 \uparrow$ & 0.006 \\
\hline$\underline{516046}$ & CD1D & CD1D antigen, d polypeptide & $24.4 \uparrow$ & 0.004 \\
\hline$\underline{511650}$ & AGGF1 & Angiogenic factor with $\mathrm{G}$ patch and FHA domains 1 & $24.1 \uparrow$ & 0.001 \\
\hline 521081 & LOC521081 & Fatty acid desaturase 2 -like protein-like & $23.1 \uparrow$ & 0.005 \\
\hline$\underline{617064}$ & OTUD6B & OTU domain containing 6B & $22.7 \uparrow$ & 0.003 \\
\hline
\end{tabular}


Table 4.6 Supplemental (continued)

\begin{tabular}{|c|c|c|c|c|}
\hline Gene ID & Symbol & Full name & $\begin{array}{l}\text { Percent } \\
\text { change }^{1}\end{array}$ & P-Value \\
\hline$\underline{505926}$ & BCAT1 & Branched chain amino-acid transaminase 1 , cytosolic & $22.2 \uparrow$ & 0.000 \\
\hline$\overline{444872}$ & HSPG2 & Heparan sulfate proteoglycan 2 & $22.2 \uparrow$ & 0.000 \\
\hline 536732 & ESRRG & Estrogen-related receptor gamma & $22.2 \uparrow$ & 0.003 \\
\hline$\underline{616701}$ & JAZF1 & $\mathrm{JAZF}$ zinc finger 1 & $22 \uparrow$ & 0.005 \\
\hline$\underline{540803}$ & MRAS & Muscle RAS oncogene homolog & $21.8 \uparrow$ & 0.018 \\
\hline$\underline{538425}$ & CTDSPL & CTD (carboxy-terminal domain, RNA polymerase II, polypeptide A) small & $21.7 \uparrow$ & 0.008 \\
\hline$\underline{536813}$ & SLC25A27 & Solute carrier family 25 , member 27 & $21.5 \uparrow$ & 0.001 \\
\hline$\underline{521867}$ & MACC1 & Metastasis associated in colon cancer 1 & $21.4 \uparrow$ & 0.002 \\
\hline$\underline{781239}$ & PLCXD3 & Phosphatidylinositol-specific phospholipase $\mathrm{C}, \mathrm{X}$ domain containing 3 & $21.2 \uparrow$ & 0.001 \\
\hline$\underline{614096}$ & ELOVL7 & ELOVL fatty acid elongase 7 & $21.1 \uparrow$ & 0.018 \\
\hline 780995 & LOC780995 & Regulator of nonsense transcripts 3B-like & $20.9 \uparrow$ & 0.001 \\
\hline$\underline{782214}$ & IL17RD & Interleukin 17 receptor D & $20.7 \uparrow$ & 0.006 \\
\hline$\underline{789588}$ & LOC789588 & Cytochrome P450, family 2, subfamily B, polypeptide 6-like & $20.3 \uparrow$ & 0.001 \\
\hline$\underline{537711}$ & $\mathrm{ABI} 2$ & Abl-interactor 2 & $20.2 \uparrow$ & 0.001 \\
\hline$\underline{532273}$ & SYT17 & Synaptotagmin XVII & $20 \uparrow$ & 0.002 \\
\hline$\underline{519541}$ & RNF125 & Ring finger protein 125 & $19.6 \uparrow$ & 0.002 \\
\hline$\underline{518246}$ & GIPC2 & GIPC PDZ domain containing family, member 2 & $19.6 \uparrow$ & 0.006 \\
\hline$\overline{100140623}$ & LOC 10014062 & Uncharacterized LOC100140623 & $19.5 \uparrow$ & 0.017 \\
\hline 517987 & NEBL & Nebulette & $19.4 \uparrow$ & 0.001 \\
\hline$\underline{614176}$ & CCDC148 & Coiled-coil domain containing 148 & $19.4 \uparrow$ & 0.004 \\
\hline$\underline{781850}$ & LOC781850 & THAP domain-containing protein 2-like & $19.3 \uparrow$ & 0.001 \\
\hline$\underline{614203}$ & GALNTL4 & UDP-N-acetyl-alpha-D-galactosamine:polypeptide N- & $19.3 \uparrow$ & 0.003 \\
\hline$\underline{519222}$ & DIS3L2 & DIS3 mitotic control homolog (S. Cerevisiae)-like 2 & $19.2 \uparrow$ & 0.004 \\
\hline$\underline{514767}$ & TGM5 & Transglutaminase 5 & $18.9 \uparrow$ & 0.006 \\
\hline$\underline{527624}$ & 4-Mar & Membrane-associated ring finger $(\mathrm{C} 3 \mathrm{HC} 4)$, $\mathrm{E} 3$ ubiquitin protein ligase & $18.7 \uparrow$ & 0.009 \\
\hline$\underline{507516}$ & SCN7A & Sodium channel, voltage-gated, type VII, alpha & $18.5 \uparrow$ & 0.005 \\
\hline
\end{tabular}


Table 4.6 Supplemental (continued)

\begin{tabular}{|c|c|c|c|c|}
\hline Gene ID & Symbol & Full name & $\begin{array}{l}\text { Percent } \\
\text { change }^{1}\end{array}$ & P-Value \\
\hline 536532 & TAOK1 & TAO kinase 1 & $18.2 \uparrow$ & 0.001 \\
\hline$\overline{521976}$ & DPY19L3 & Dpy-19-like 3 (C. Elegans) & $18.1 \uparrow$ & 0.001 \\
\hline$\underline{617339}$ & FBXO36 & F-box protein 36 & $17.5 \uparrow$ & 0.001 \\
\hline$\overline{507285}$ & CHN2 & Chimerin (chimaerin) 2 & $17.5 \uparrow$ & 0.003 \\
\hline 281062 & $\mathrm{CDH} 2$ & Cadherin 2, type $1, \mathrm{~N}$-cadherin (neuronal) & $17.3 \uparrow$ & 0.004 \\
\hline$\underline{533191}$ & TMTC1 & Transmembrane and tetratricopeptide repeat containing 1 & $17.3 \uparrow$ & 0.006 \\
\hline$\underline{521959}$ & HS3ST3A1 & Heparan sulfate (glucosamine) 3-O-sulfotransferase 3A1 & $17.3 \uparrow$ & 0.008 \\
\hline$\underline{100336428}$ & LOC10033642 & Uncharacterized LOC100336428 & $17.2 \uparrow$ & 0.011 \\
\hline$\underline{516032}$ & SMAP1 & Small arfgap 1 & $17.1 \uparrow$ & 0.004 \\
\hline$\underline{100296635}$ & USH2A & Usher syndrome 2A (autosomal recessive, mild) & $17.1 \uparrow$ & 0.005 \\
\hline$\underline{535872}$ & SLC16A2 & Solute carrier family 16, member 2 (monocarboxylic acid transporter 8 ) & $16.7 \uparrow$ & 0.001 \\
\hline$\underline{519342}$ & EBF2 & Early B-cell factor 2 & $16.7 \uparrow$ & 0.011 \\
\hline$\underline{768230}$ & CYYR1 & Cysteine/tyrosine-rich 1 & $16.4 \uparrow$ & 0.001 \\
\hline$\underline{613849}$ & COL13A1 & Collagen, type XIII, alpha 1 & $16.1 \uparrow$ & 0.014 \\
\hline$\underline{540275}$ & CHRDL1 & Chordin-like 1 & $15.9 \uparrow$ & 0.011 \\
\hline$\underline{513653}$ & WDR52 & WD repeat domain 52 & $15.7 \uparrow$ & 0.003 \\
\hline$\underline{533796}$ & BICD1 & Bicaudal D homolog 1 (Drosophila) & $15.7 \uparrow$ & 0.006 \\
\hline$\underline{788352}$ & NKAIN3 & $\mathrm{Na}+/ \mathrm{K}+$ transporting atpase interacting 3 & $15.6 \uparrow$ & 0.004 \\
\hline$\underline{615452}$ & TBC1D19 & TBC1 domain family, member 19 & $15.6 \uparrow$ & 0.005 \\
\hline$\underline{519310}$ & EPB41L4B & Erythrocyte membrane protein band 4.1 like $4 \mathrm{~B}$ & $15.5 \uparrow$ & 0.004 \\
\hline 493725 & DDX4 & DEAD (Asp-Glu-Ala-Asp) box polypeptide 4 & $15.2 \uparrow$ & 0.018 \\
\hline$\underline{508289}$ & OR6S1 & Olfactory receptor, family 6 , subfamily $S$, member 1 & $15.1 \uparrow$ & 0.030 \\
\hline$\underline{539366}$ & LRFN5 & Leucine rich repeat and fibronectin type III domain containing 5 & $15 \uparrow$ & 0.005 \\
\hline$\underline{508204}$ & GREB1 & Growth regulation by estrogen in breast cancer 1 & $14.8 \uparrow$ & 0.005 \\
\hline 100337015 & LOC10033701 & Rho guanine nucleotide exchange factor (GEF) 12-like & $14.8 \uparrow$ & 0.006 \\
\hline$\underline{512981}$ & EPB49 & Erythrocyte membrane protein band 4.9 (dematin) & $14.7 \uparrow$ & 0.032 \\
\hline
\end{tabular}


Table 4.6 Supplemental (continued)

\begin{tabular}{|c|c|c|c|c|}
\hline Gene ID & Symbol & Full name & $\begin{array}{l}\text { Percent } \\
\text { change }^{1}\end{array}$ & P-Value \\
\hline$\underline{525878}$ & EFCAB5 & EF-hand calcium binding domain 5 & $14.6 \uparrow$ & 0.002 \\
\hline$\overline{781953}$ & MATN2 & Matrilin 2 & $14.6 \uparrow$ & 0.007 \\
\hline$\underline{100336913}$ & $\mathrm{ABCB} 4$ & ATP-binding cassette, sub-family B (MDR/TAP), member 4 & $14.3 \uparrow$ & 0.019 \\
\hline$\overline{512781}$ & ARL13B & ADP-ribosylation factor-like 13B & $14.3 \uparrow$ & 0.022 \\
\hline$\overline{782935}$ & FAM19A1 & Family with sequence similarity 19 (chemokine (C-C motif)-like), member A1 & $14.2 \uparrow$ & 0.009 \\
\hline$\underline{100140203}$ & DTHD1 & Death domain containing 1 & $14.2 \uparrow$ & 0.029 \\
\hline$\underline{537580}$ & LOC537580 & Leucine-rich repeat-containing protein 9-like & $14 \uparrow$ & 0.005 \\
\hline Top of & C3H1orf192 & Chromosome 3 open reading frame, human c1orf192 & $13.7 \uparrow$ & 0.014 \\
\hline$\underline{614302}$ & PCCA & Propionyl coa carboxylase, alpha polypeptide & $13.6 \uparrow$ & 0.007 \\
\hline$\underline{777690}$ & LRRC17 & Leucine rich repeat containing 17 & $13.6 \uparrow$ & 0.035 \\
\hline$\underline{617365}$ & EFCAB11 & EF-hand calcium binding domain 11 & $13.4 \uparrow$ & 0.004 \\
\hline$\underline{534683}$ & COMMD1 & Copper metabolism (Murr1) domain containing 1 & $13.4 \uparrow$ & 0.005 \\
\hline$\underline{281941}$ & NCAM1 & Neural cell adhesion molecule 1 & $13.4 \uparrow$ & 0.015 \\
\hline$\underline{508266}$ & CHN1 & Chimerin (chimaerin) 1 & $13 \uparrow$ & 0.007 \\
\hline$\underline{100336965}$ & ODZ1 & Odz, odd Oz/ten-m homolog 1 (Drosophila) & $12.9 \uparrow$ & 0.007 \\
\hline 282049 & NMT2 & $\mathrm{N}$-myristoyltransferase 2 & $12.8 \uparrow$ & 0.030 \\
\hline$\underline{511034}$ & TMEM108 & Transmembrane protein 108 & $12.6 \uparrow$ & 0.011 \\
\hline 282124 & VSNL1 & Visinin-like 1 & $12.5 \uparrow$ & 0.024 \\
\hline$\underline{615715}$ & EFCAB2 & EF-hand calcium binding domain 2 & $12.3 \uparrow$ & 0.007 \\
\hline$\underline{522122}$ & SMC1B & Structural maintenance of chromosomes 1B & $12.1 \uparrow$ & 0.019 \\
\hline$\underline{524491}$ & CUX2 & Cut-like homeobox 2 & $12 \uparrow$ & 0.010 \\
\hline$\underline{539968}$ & LINGO2 & Leucine rich repeat and Ig domain containing 2 & $11.8 \uparrow$ & 0.003 \\
\hline$\underline{540817}$ & MOB3B & MOB kinase activator 3B & $11.3 \uparrow$ & 0.009 \\
\hline$\overline{509483}$ & CEP135 & Centrosomal protein $135 \mathrm{kda}$ & $11.2 \uparrow$ & 0.002 \\
\hline$\underline{519892}$ & NUAK1 & NUAK family, SNF1-like kinase, 1 & $11.1 \uparrow$ & 0.038 \\
\hline$\underline{539650}$ & IGF2BP3 & Insulin-like growth factor 2 mrna binding protein 3 & $10.4 \uparrow$ & 0.012 \\
\hline
\end{tabular}


Table 4.6 Supplemental (continued)

\begin{tabular}{|c|c|c|c|c|}
\hline Gene ID & Symbol & Full name & $\begin{array}{l}\text { Percent } \\
\text { change }^{1}\end{array}$ & P-Value \\
\hline$\underline{617013}$ & LOC617013 & Uncharacterized LOC617013 & $10.3 \uparrow$ & 0.018 \\
\hline$\overline{100139725}$ & ZDBF2 & Zinc finger, DBF-type containing 2 & $10.3 \uparrow$ & 0.023 \\
\hline$\overline{541161}$ & DKK2 & Dickkopf homolog 2 (Xenopus laevis) & $10.1 \uparrow$ & 0.016 \\
\hline$\overline{524180}$ & CSPP1 & Centrosome and spindle pole associated protein 1 & $9.3 \uparrow$ & 0.023 \\
\hline 783452 & LOC783452 & Pro-neuregulin-2, membrane-bound isoform-like & $7.7 \uparrow$ & 0.035 \\
\hline 768023 & C7H19orf71 & Chromosome 7 open reading frame, human c19orf71 & $62.5 \downarrow$ & 0.002 \\
\hline$\underline{513599}$ & TMEM79 & Transmembrane protein 79 & $58 \downarrow$ & 0.001 \\
\hline$\underline{281286}$ & LYL1 & Lymphoblastic leukemia derived sequence 1 & $52.3 \downarrow$ & 0.007 \\
\hline$\underline{100125876}$ & $\mathrm{C} 3 \mathrm{H} 1$ orf 85 & Chromosome 3 open reading frame, human c1orf85 & $48.9 \downarrow$ & 0.006 \\
\hline$\underline{618888}$ & SAYSD1 & SAYSVFN motif domain containing 1 & $46.8 \downarrow$ & 0.000 \\
\hline$\underline{515527}$ & TMEM150B & Transmembrane protein $150 \mathrm{~B}$ & $46.6 \downarrow$ & 0.049 \\
\hline$\underline{100138621}$ & ZNF513 & Zinc finger protein 513 & $46.2 \downarrow$ & 0.001 \\
\hline 511013 & LYSMD2 & Lysm, putative peptidoglycan-binding, domain containing 2 & $44.6 \downarrow$ & 0.001 \\
\hline$\underline{526369}$ & LENG9 & Leukocyte receptor cluster (LRC) member 9 & $43.1 \downarrow$ & 0.001 \\
\hline$\underline{506467}$ & TREM2 & Triggering receptor expressed on myeloid cells 2 & $42.8 \downarrow$ & 0.032 \\
\hline$\underline{617546}$ & C23H6orf47 & Chromosome 23 open reading frame, human c6orf 47 & $40.1 \downarrow$ & 0.000 \\
\hline$\underline{505837}$ & ZNF358 & Zinc finger protein 358 & $39.6 \downarrow$ & 0.001 \\
\hline$\underline{514104}$ & WDR81 & WD repeat domain 81 & $39.6 \downarrow$ & 0.008 \\
\hline$\underline{539436}$ & ITPRIPL1 & Inositol 1,4,5-trisphosphate receptor interacting protein-like 1 & $39.5 \downarrow$ & 0.018 \\
\hline$\underline{513167}$ & GLT25D1 & Glycosyltransferase 25 domain containing 1 & $38.9 \downarrow$ & 0.002 \\
\hline$\underline{510325}$ & KIAA1539 & KIAA1539 ortholog & $37.8 \downarrow$ & 0.001 \\
\hline$\overline{518544}$ & $\mathrm{CNO}$ & Cappuccino homolog (mouse) & $37.4 \downarrow$ & 0.002 \\
\hline$\underline{281915}$ & MMP14 & Matrix metallopeptidase 14 (membrane-inserted) & $36.6 \downarrow$ & 0.021 \\
\hline$\underline{618444}$ & C11H9orf167 & Chromosome 11 open reading frame, human c9orf167 & $36.4 \downarrow$ & 0.001 \\
\hline$\underline{616482}$ & CAPS & Calcyphosine & $36.4 \downarrow$ & 0.008 \\
\hline$\underline{615572}$ & MID1IP1 & MID1 interacting protein 1 (gastrulation specific G12 homolog (zebrafish)) & $36.1 \downarrow$ & 0.001 \\
\hline
\end{tabular}


Table 4.6 Supplemental (continued)

\begin{tabular}{|c|c|c|c|c|}
\hline Gene ID & Symbol & Full name & $\begin{array}{l}\text { Percent } \\
\text { change }^{1}\end{array}$ & P-Value \\
\hline$\underline{510831}$ & NRSN1 & Neurensin 1 & $35.5 \downarrow$ & 0.000 \\
\hline$\overline{618368}$ & SPATA2L & Spermatogenesis associated 2-like & $35.4 \downarrow$ & 0.009 \\
\hline$\overline{767913}$ & GDPD3 & Glycerophosphodiester phosphodiesterase domain containing 3 & $35 \downarrow$ & 0.001 \\
\hline$\overline{507426}$ & LOC507426 & Poly [ADP-ribose] polymerase 3-like & $33.9 \downarrow$ & 0.012 \\
\hline$\underline{535935}$ & LATS1 & LATS, large tumor suppressor, homolog 1 (Drosophila) & $33.4 \downarrow$ & 0.000 \\
\hline$\underline{618605}$ & SPHK1 & Sphingosine kinase 1 & $32.9 \downarrow$ & 0.031 \\
\hline$\underline{540610}$ & CHCHD1 & Coiled-coil-helix-coiled-coil-helix domain containing 1 & $32.4 \downarrow$ & 0.011 \\
\hline$\underline{777786}$ & ATXN7L3B & Ataxin 7-like 3B & $32.1 \downarrow$ & 0.015 \\
\hline$\underline{511098}$ & LGALS2 & Lectin, galactoside-binding, soluble, 2 & $31.7 \downarrow$ & 0.015 \\
\hline$\underline{539774}$ & PHF23 & PHD finger protein 23 & $31.4 \downarrow$ & 0.001 \\
\hline$\underline{538662}$ & LSM2 & LSM2 homolog, U6 small nuclear RNA associated (S. Cerevisiae) & $31.4 \downarrow$ & 0.007 \\
\hline$\underline{782177}$ & LOC782177 & Vacuolar protein sorting-associated protein 35 -like & $31.3 \downarrow$ & 0.005 \\
\hline$\overline{508124}$ & LOC508124 & Homer protein homolog 3-like & $30.5 \downarrow$ & 0.002 \\
\hline$\underline{540925}$ & $\mathrm{SCN} 3 \mathrm{~B}$ & Sodium channel, voltage-gated, type III, beta & $30.3 \downarrow$ & 0.019 \\
\hline$\underline{404144}$ & LMNA & Lamin $\mathrm{A} / \mathrm{C}$ & $30.1 \downarrow$ & 0.011 \\
\hline$\underline{281555}$ & TUBB2A & Tubulin, beta $2 \mathrm{~A}$ class iia & $29.6 \downarrow$ & 0.001 \\
\hline$\underline{512667}$ & ADAL & Adenosine deaminase-like & $29.5 \downarrow$ & 0.006 \\
\hline$\underline{535407}$ & $\mathrm{ACP} 2$ & Acid phosphatase 2, lysosomal & $28.9 \downarrow$ & 0.011 \\
\hline$\underline{617482}$ & KBTBD4 & Kelch repeat and BTB (POZ) domain containing 4 & $28.8 \downarrow$ & 0.022 \\
\hline$\underline{505571}$ & TSEN34 & Trna splicing endonuclease 34 homolog (S. Cerevisiae) & $28.5 \downarrow$ & 0.004 \\
\hline$\underline{511011}$ & CXHXorf26 & Chromosome $X$ open reading frame, human cxorf 26 & $28.1 \downarrow$ & 0.011 \\
\hline$\overline{531644}$ & C1GALT1C1 & C1GALT1-specific chaperone 1 & $28 \downarrow$ & 0.000 \\
\hline$\underline{100300734}$ & FAM189B & Family with sequence similarity 189 , member B & $27.9 \downarrow$ & 0.007 \\
\hline 281555 & TUBB2A & Tubulin, beta $2 \mathrm{~A}$ class iia & $27.8 \downarrow$ & 0.004 \\
\hline$\underline{535768}$ & MTO1 & Mitochondrial translation optimization 1 homolog (S. Cerevisiae) & $27.8 \downarrow$ & 0.006 \\
\hline$\underline{511091}$ & TSEN54 & Trna splicing endonuclease 54 homolog (S. Cerevisiae) & $27.6 \downarrow$ & 0.015 \\
\hline
\end{tabular}


Table 4.6 Supplemental (continued)

\begin{tabular}{|c|c|c|c|c|}
\hline Gene ID & Symbol & Full name & $\begin{array}{l}\text { Percent } \\
\text { change }^{1}\end{array}$ & P-Value \\
\hline$\underline{531984}$ & FAM86A & Family with sequence similarity 86 , member A & $27.4 \downarrow$ & 0.005 \\
\hline$\overline{521401}$ & AMDHD2 & Amidohydrolase domain containing 2 & $26.5 \downarrow$ & 0.001 \\
\hline$\underline{509037}$ & TMX1 & Thioredoxin-related transmembrane protein 1 & $26.2 \downarrow$ & 0.017 \\
\hline$\underline{513723}$ & RALGDS & Ral guanine nucleotide dissociation stimulator & $26 \downarrow$ & 0.006 \\
\hline$\underline{100300220}$ & LOC10030022 & Alpha isoform of regulatory subunit A, protein phosphatase 2-like pseudogene & $26 \downarrow$ & 0.027 \\
\hline$\underline{511926}$ & MRPL54 & Mitochondrial ribosomal protein L54 & $25.8 \downarrow$ & 0.003 \\
\hline$\underline{515465}$ & ICT1 & Immature colon carcinoma transcript 1 & $25.8 \downarrow$ & 0.017 \\
\hline$\underline{616015}$ & C11H9orf16 & Chromosome 11 open reading frame, human c9orf16 & $25.7 \downarrow$ & 0.014 \\
\hline$\underline{507930}$ & LOC507930 & Ras-related GTP-binding protein C-like & $25.1 \downarrow$ & 0.009 \\
\hline$\underline{507921}$ & MFSD5 & Major facilitator superfamily domain containing 5 & $24.8 \downarrow$ & 0.001 \\
\hline$\underline{508965}$ & C7H19orf25 & Chromosome 7 open reading frame, human c19orf 25 & $24.7 \downarrow$ & 0.018 \\
\hline$\underline{512312}$ & PTPN6 & Protein tyrosine phosphatase, non-receptor type 6 & $24.6 \downarrow$ & 0.019 \\
\hline$\underline{509053}$ & NKIRAS2 & NFKB inhibitor interacting Ras-like 2 & $24.5 \downarrow$ & 0.012 \\
\hline$\underline{504517}$ & BABAM1 & BRISC and BRCA1 A complex member 1 & $24.5 \downarrow$ & 0.024 \\
\hline$\underline{507664}$ & TRPV2 & Transient receptor potential cation channel, subfamily V, member 2 & $23.8 \downarrow$ & 0.011 \\
\hline$\underline{505738}$ & MCOLN1 & Mucolipin 1 & $23.4 \downarrow$ & 0.017 \\
\hline$\underline{531211}$ & FBXL14 & F-box and leucine-rich repeat protein 14 & $23.3 \downarrow$ & 0.005 \\
\hline$\underline{617367}$ & RRAGC & Ras-related GTP binding C & $22.5 \downarrow$ & 0.005 \\
\hline$\underline{534248}$ & MGAT1 & Mannosyl (alpha-1,3-)-glycoprotein beta-1,2-N-acetylglucosaminyltransferase & $22.3 \downarrow$ & 0.012 \\
\hline$\underline{617650}$ & GCHFR & GTP cyclohydrolase I feedback regulator & $22.2 \downarrow$ & 0.04 \\
\hline$\underline{507473}$ & CNPPD1 & Cyclin Pas1/PHO80 domain containing 1 & $22.1 \downarrow$ & 0.006 \\
\hline$\underline{767834}$ & KLHL9 & Kelch-like 9 (Drosophila) & $22.1 \downarrow$ & 0.042 \\
\hline$\underline{538537}$ & ZNF7 & Zinc finger protein 7 & $22 \downarrow$ & 0.022 \\
\hline$\underline{504961}$ & MPDU1 & Mannose-P-dolichol utilization defect 1 & $21.9 \downarrow$ & 0.001 \\
\hline$\underline{511816}$ & SNAPIN & SNAP-associated protein & $21.7 \downarrow$ & 0.017 \\
\hline$\underline{534282}$ & RNF145 & Ring finger protein 145 & $21.2 \downarrow$ & 0.022 \\
\hline
\end{tabular}


Table 4.6 Supplemental (continued)

\begin{tabular}{|c|c|c|c|c|}
\hline Gene ID & Symbol & Full name & $\begin{array}{l}\text { Percent } \\
\text { change }^{1}\end{array}$ & P-Value \\
\hline$\underline{614209}$ & LOC614209 & Developmentally regulated GTP binding protein 1 pseudogene & $21 \downarrow$ & 0.002 \\
\hline$\underline{514858}$ & TBC1D17 & TBC1 domain family, member 17 & $20.5 \downarrow$ & 0.007 \\
\hline$\underline{100125239}$ & CCDC127 & Coiled-coil domain containing 127 & $20.3 \downarrow$ & 0.011 \\
\hline 783569 & CSTF2T & Cleavage stimulation factor, $3^{\prime}$ pre-RNA, subunit $2,64 \mathrm{kda}$, tau variant & $20.2 \downarrow$ & 0.004 \\
\hline$\overline{515389}$ & MTA2 & Metastasis associated 1 family, member 2 & $19.9 \downarrow$ & 0.013 \\
\hline$\underline{531673}$ & CASC3 & Cancer susceptibility candidate 3 & $19.5 \downarrow$ & 0.005 \\
\hline 497032 & MTHFR & Methylenetetrahydrofolate reductase (NAD(P)H) & $19.4 \downarrow$ & 0.014 \\
\hline$\underline{613932}$ & PLD3 & Phospholipase D family, member 3 & $19.2 \downarrow$ & 0.020 \\
\hline$\underline{338042}$ & SLC35A2 & Solute carrier family 35 (UDP-galactose transporter), member A2 & $18.9 \downarrow$ & 0.005 \\
\hline$\underline{767983}$ & SDHAF2 & Succinate dehydrogenase complex assembly factor 2 & $18.9 \downarrow$ & 0.009 \\
\hline$\underline{505039}$ & GATAD2B & GATA zinc finger domain containing 2B & $18.8 \downarrow$ & 0.009 \\
\hline$\underline{100140430}$ & DDX42 & DEAD (Asp-Glu-Ala-Asp) box polypeptide 42 & $18.8 \downarrow$ & 0.013 \\
\hline$\underline{509419}$ & HMGXB3 & HMG box domain containing 3 & $18.8 \downarrow$ & 0.027 \\
\hline$\underline{506370}$ & CYB5B & Cytochrome b5 type B (outer mitochondrial membrane) & $18.7 \downarrow$ & 0.009 \\
\hline$\underline{505570}$ & C13H20orf11 & Chromosome 13 open reading frame, human c20orf11 & $17.2 \downarrow$ & 0.017 \\
\hline$\underline{508235}$ & FBXO7 & F-box protein 7 & $17.1 \downarrow$ & 0.015 \\
\hline$\underline{618512}$ & HN1L & Hematological and neurological expressed 1-like & $17.1 \downarrow$ & 0.031 \\
\hline$\underline{768068}$ & KLHL12 & Kelch-like 12 (Drosophila) & $17 \downarrow$ & 0.031 \\
\hline$\underline{510698}$ & VAT1 & Vesicle amine transport protein 1 homolog (T. Californica) & $16.8 \downarrow$ & 0.023 \\
\hline$\underline{509488}$ & SEC24C & SEC24 family, member C (S. Cerevisiae) & $16.5 \downarrow$ & 0.015 \\
\hline$\underline{513510}$ & GTF2H1 & General transcription factor $\mathrm{IIH}$, polypeptide $1,62 \mathrm{kda}$ & $16.4 \downarrow$ & 0.006 \\
\hline$\overline{614212}$ & LMO4 & LIM domain only 4 & $16.4 \downarrow$ & 0.023 \\
\hline$\underline{615905}$ & ZNF34 & Zinc finger protein 34 & $16.2 \downarrow$ & 0.008 \\
\hline$\underline{506358}$ & MRPL20 & Mitochondrial ribosomal protein L20 & $16 \downarrow$ & 0.037 \\
\hline$\underline{541149}$ & LOC541149 & Transmembrane protein 185A-like & $15.9 \downarrow$ & 0.012 \\
\hline 525164 & TRIAP1 & TP53 regulated inhibitor of apoptosis 1 & $15.8 \downarrow$ & 0.029 \\
\hline
\end{tabular}


Table 4.6 Supplemental (continued)

\begin{tabular}{|c|c|c|c|c|}
\hline Gene ID & Symbol & Full name & $\begin{array}{l}\text { Percent } \\
\text { change }^{1}\end{array}$ & P-Value \\
\hline$\underline{783336}$ & WDR82 & WD repeat domain 82 & $15.3 \downarrow$ & 0.002 \\
\hline$\overline{100297865}$ & ACTR5 & ARP5 actin-related protein 5 homolog (yeast) & $15.3 \downarrow$ & 0.025 \\
\hline 504807 & COMMD5 & COMM domain containing 5 & $15.3 \downarrow$ & 0.025 \\
\hline$\overline{514789}$ & ISCU & Iron-sulfur cluster scaffold homolog (E. Coli) & $15 \downarrow$ & 0.034 \\
\hline$\underline{100299693}$ & PRPF38B & PRP38 pre-mrna processing factor 38 (yeast) domain containing B & $14.8 \downarrow$ & 0.012 \\
\hline$\underline{767979}$ & HSBP1 & Heat shock factor binding protein 1 & $14.5 \downarrow$ & 0.016 \\
\hline$\underline{511329}$ & PARP6 & Poly (ADP-ribose) polymerase family, member 6 & $14.5 \downarrow$ & 0.030 \\
\hline$\underline{539294}$ & PPCS & Phosphopantothenoylcysteine synthetase & $14.1 \downarrow$ & 0.020 \\
\hline$\overline{521864}$ & VPS35 & Vacuolar protein sorting 35 homolog (S. Cerevisiae) & $14.1 \downarrow$ & 0.033 \\
\hline$\overline{518880}$ & RBX1 & Ring-box 1, E3 ubiquitin protein ligase & $14 \downarrow$ & 0.046 \\
\hline$\underline{613828}$ & DR1 & Down-regulator of transcription 1, TBP-binding (negative cofactor 2) & $13.7 \downarrow$ & 0.032 \\
\hline$\overline{537087}$ & GBA & Glucosidase, beta, acid & $13.6 \downarrow$ & 0.05 \\
\hline$\overline{540348}$ & ELOVL1 & ELOVL fatty acid elongase 1 & $13.3 \downarrow$ & 0.045 \\
\hline$\overline{509725}$ & DIMT1 & DIM1 dimethyladenosine transferase 1 homolog (S. Cerevisiae) & $12.9 \downarrow$ & 0.047 \\
\hline 767822 & PNPLA6 & Patatin-like phospholipase domain containing 6 & $12.8 \downarrow$ & 0.042 \\
\hline 281691 & DNAJC14 & Dnaj (Hsp40) homolog, subfamily C, member 14 & $12.5 \downarrow$ & 0.015 \\
\hline$\underline{518859}$ & NUDT21 & Nudix (nucleoside diphosphate linked moiety X)-type motif 21 & $11.8 \downarrow$ & 0.029 \\
\hline$\underline{517097}$ & CTCF & CCCTC-binding factor (zinc finger protein) & $11.8 \downarrow$ & 0.036 \\
\hline$\overline{511803}$ & PSMD1 & Proteasome (prosome, macropain) 26S subunit, non-atpase, 1 & $11.4 \downarrow$ & 0.023 \\
\hline$\underline{530189}$ & RAD23B & RAD23 homolog B (S. Cerevisiae) & $10.6 \downarrow$ & 0.022 \\
\hline$\overline{617316}$ & ELAVL1 & ELAV (embryonic lethal, abnormal vision, Drosophila)-like 1 (Hu antigen R) & $10.5 \downarrow$ & 0.026 \\
\hline$\overline{513824}$ & TMEM128 & Transmembrane protein 128 & $10.2 \downarrow$ & 0.027 \\
\hline$\overline{529700}$ & RPRD1B & Regulation of nuclear pre-mrna domain containing $1 \mathrm{~B}$ & $10.1 \downarrow$ & 0.031 \\
\hline
\end{tabular}

${ }^{\mathrm{I}}$ Direction of change in expression of genes is indicated with a $\uparrow$ or $\downarrow$ 


\title{
CHAPTER FIVE
}

\section{EFFECT OF TRACE MINERAL SUPPLEMENTATION SOURCE ON THE FUNCTION OF NEUTROPHILS COLLECTED FROM PERIPARTURIENT DAIRY COWS}

\begin{abstract}
The objective of this study was to investigate the effects of trace mineral supplementation from inorganic or organic sources on neutrophil (PMNL) function in dairy cows during the periparturient period. Thirty nine pregnant Holstein cows entering second or greater lactation were randomly assigned to a basal diet with no added dietary $\mathrm{Mn}, \mathrm{Co}, \mathrm{Cu}$ and $\mathrm{Zn}$ (basal, $\mathrm{n}=13$ ) or orally supplemented via gelcap with $200 \mathrm{mg} \mathrm{Mn}$, $25 \mathrm{mg} \mathrm{Co}, 125 \mathrm{mg} \mathrm{Cu}$, and $360 \mathrm{mg} \mathrm{Zn}$ from sulfate and carbonate sources (inorganic, $\mathrm{n}=$ 11) or supplied by amino acid complexes of $\mathrm{Mn}, \mathrm{Cu}$, and $\mathrm{Zn}$, and cobalt glucoheptonate (organic, $\mathrm{n}=11$ ). Total supplementation for inorganic and organic cows was brought to $65,15,75$, and $2.5 \mathrm{ppm}$ of diet using the inorganic sources added to each gelcap for Mn, $\mathrm{Cu}, \mathrm{Zn}$, and $\mathrm{Co}$, respectively. Oral treatments were administered between day $57.1 \pm 1$ prior to parturition until day 8 after parturition. PMNL were collected from the blood of cows on days $58.1 \pm 1.0$ (pre-treatment sample), $7.3 \pm 0.7$ prior to parturition and $6.4 \pm$ 0.1 after parturition. To investigate the effects of trace mineral supplementation on basal and stimulated PMNL function, the cells were incubated with 0 or $50 \mu \mathrm{g} / \mathrm{mL}$ of Escherichia coli lipopolysaccharide (LPS) for $120 \mathrm{~min}$. Subsequently, the generation of reactive oxygen species (ROS), PMNL extracellular traps (NETs), chemotaxis towards interleukin-8 (IL-8), phagocytosis of E. coli particles, and killing ability against E. coli
\end{abstract}


were determined. Cows supplemented with trace minerals from inorganic sources had a $39 \%$ increase in the amount of E. coli particles phagocytized by PMNL on day 6 postpartum, compared with cows receiving no supplemental trace minerals. There was no difference in any other parameter of PMNL function between animals supplemented with inorganic or organic forms of trace minerals and those receiving no supplementation. Replacing inorganic forms of trace mineral with the same amount of trace minerals from organic sources had no effect on any aspect of PMNL function. Independently of dietary treatment, PMNL production of ROS decreased by $38 \%$ between days 58 prepartum and 6 postpartum. In addition, PMNL chemotaxis towards IL- 8 declined by $18 \%$ between days 58 prepartum and 6 postpartum, but did not differ between days 7 prepartum and 6 postpartum. The percentage of PMNL positive for phagocytized E. coli particles and their mean fluorescence intensity decreased between days 58 and 7 prepartum and then slightly recovered by day 6 postpartum. Conversely, PMNL NETs release and killing of E. coli augmented as the animals transitioned from the prepartum into the postpartum period. In summary, replacing trace minerals from inorganic sources with more bioavailable forms such as those from organic sources had no effect on PMNL function. Regardless of trace mineral supplementation, the production of ROS, chemotaxis towards IL-8, and phagocytosis of $E$. coli particles by PMNL were compromised in the periparturient period and may contribute to the increased incidence and severity of IMI observed around the time of parturition. 


\section{INTRODUCTION}

Dairy cows are more susceptible to infectious diseases in the mammary gland for several weeks around the time of parturition in part due to an incompetent immune system and increased oxidative stress (Mallard et al., 1998; Sordillo and Aitken, 2009). The incapacity of local defense mechanisms to effectively detect and eliminate the invading pathogen during this period may contribute to the increased incidence and severity of intramammary infections (IMI; Mallard et al., 1998). In the mammary gland of dairy cows, the innate immune system is the most important host defense mechanism against the invading pathogen (Burvenich et al., 2007). Polymorphonuclear leukocytes or neutrophils (PMNL) are key components of the innate immune response to IMI. Although macrophages comprise the majority of leukocytes present in the healthy mammary gland, PMNL migrate rapidly towards to the gland in the case of an IMI and represent the predominant cellular population in the infected gland for several days (Saad and Ostensson, 1990). In the infected mammary gland, the primary roles of PMNL are the recognition, containment, and killing of the invading pathogen. Bovine PMNL use several strategies to destroy pathogens including microbial uptake, intracellular killing and the secretion of antimicrobial molecules (e.g. reactive oxygen species or ROS) to the extracellular space (Paape et al., 2003). PMNL are also capable of releasing granule proteins and de-condensed chromatin to form extracellular fibers known as neutrophil extracellular traps (NETs) that bind microorganisms, preventing them from distributing throughout the tissue, and killing the pathogens by keeping them in a high local concentration of antimicrobial agents (Brinkmann et al., 2004). Formation and release of NETs involves a novel type of cell death program distinct from apoptosis and necrosis 
that requires autophagy and activation of NADPH oxidase (Remijsen et al., 2011). The successful migration of PMNL into the mammary gland via chemotaxis and subsequent phagocytosis and killing are crucial for the resolution of IMI (Vangroenweghe et al., 2005). However, the antimicrobial capacity of PMNL is altered during the periparturient period and may contribute to the increased incidence and severity of infectious disease (Kehrli et al., 1989; Cai et al., 1994; Revelo and Waldron, 2010). At the same time, uncontrolled influx of PMNL into the mammary gland may lead to severe host tissue damage as a result of accumulation of toxic levels of ROS and cytokines (Zhao and Lacasse, 2008). Thus, a delicate balance must exist between a vigorous immune response required to eliminate the invading pathogen and the activation of anti-inflammatory mechanisms, indispensable to reinstate mammary gland immune homeostasis (Aitken et al., 2011).

Several micronutrients including trace minerals have been demonstrated to influence the immune response and disease resistance of cattle (Spears, 2000). Trace minerals are structural components and cofactors of enzymes involved in the antioxidant defense system (Spears and Weiss, 2008). In addition to their traditional role in the generation of anti-oxidants, trace minerals such as $\mathrm{Zn}$ can directly influence immune cell function via regulation of gene expression, hematopoiesis and cytokine production (Haase and Rink, 2009; Prasad, 2007). The protective role by antioxidant mechanisms is essential during the periparturient period when the dairy cow experiences increased oxidative stress (Bernabucci et al., 2005; Sordillo et al., 2007). Excessive generation of oxygen radicals by activated phagocytes may cause mammary epithelial cell damage and compromise its function (Lauzon et al., 2005; Zhao and Lacasse, 2008). Alternatively, 
oxidative stress may impair the protective functions of the immune cells via increased lipid peroxidation of polyunsaturated fatty acids and reduced membrane fluidity (Bendich, 1993). Thus, deficiencies of trace minerals with antioxidants may contribute to altered immune function in transition dairy cows (Spears and Weiss, 2008).

Adequate trace mineral nutrition is needed to optimize the health of dairy cows during the periparturient period when the immune and inflammatory systems are less efficient (Andrieu, 2008). In dairy cows, supplementation of trace minerals has traditionally relied on inorganic salts including sulfates, oxides, and carbonates (Andrieu, 2008). However, antagonism between metal ions, microbial digestion, and negative interactions with fiber and certain sugars may reduce the bioavailability of trace minerals in these forms (Andrieu, 2008; Spears, 2003). Conversely, trace minerals from organic sources resist many of these interactions increasing their bioavailability compared with minerals from inorganic sources (Predieri et al., 2005; Wedekind et al., 1992). In dairy cows, benefits from replacing $\mathrm{Mn}, \mathrm{Cu}$, and $\mathrm{Zn}$, and $\mathrm{Co}$ from inorganic sources with equivalent amounts of these trace minerals as organic complexes include higher milk yield (Ballantine et al., 2002; Hackbart et al., 2010; Nocek et al., 2006), increased milk fat content (Formigoni et al., 2011; Siciliano-Jones et al., 2008), augmented total immunoglobulins (Ig) content in colostrum (Formigoni et al., 2011), and decreased the incidence of sole ulcers (Siciliano-Jones et al., 2008). Furthermore, it has been demonstrated that dairy cattle fed diets deficient in Co and Cu have PMNL with reduced antimicrobial capacity in vitro (MacPherson et al., 1987; Xin et al., 1991; Scaletti et al., 2003). However, the influence of dietary supplementation of trace minerals from organic sources on PMNL has not been investigated in periparturient dairy cows. Therefore, the 
objective of this study was to determine the effects of trace mineral supplementation from either inorganic or organic sources on PMNL function in dairy cows during the periparturient period. We hypothesized that cows supplemented with amino acid complexes of $\mathrm{Mn}, \mathrm{Cu}$, and $\mathrm{Zn}$, and Co glucoheptonate would have PMNL with enhanced generation of ROS, release of NETs, chemotaxis, phagocytosis and killing abilities, relative to animals supplemented with the same amount of trace minerals from inorganic sources.

\section{MATERIALS AND METHODS}

\section{Experimental Design, Animals, and Treatments}

The use and care of all animals were approved by The University of Missouri Animal Care and Use Committee. This experiment was conducted as a randomized complete block design with cow as the experimental unit. Thirty nine pregnant Holstein cows entering second or greater lactation were randomly assigned to one of 13 blocks according to average body condition score (BCS), previous lactation somatic cell count (SCC) and previous lactation ME 305 milk production. All cows enrolled in the study did not experience any major clinical event during the previous lactation, had an average previous lactation somatic cell count lower than 300,000 cells/ml and a current locomotion score lower than two (Sprecher et al., 1997). Four cows developed clinical health complications diagnosed by staff veterinarians during the experiment and therefore removed from the study. All cows were fed a basal diet formulated to meet or exceed nutritional recommendations for all nutrients except $\mathrm{Mn}, \mathrm{Co}, \mathrm{Cu}$, and $\mathrm{Zn}$ (Table 5.1; NRC, 2001). Cows within each block were assigned to one of three treatments: no added 
dietary $\mathrm{Mn}, \mathrm{Co}, \mathrm{Cu}$ and $\mathrm{Zn}$ (basal, $\mathrm{n}=13$ ), additional $\mathrm{Mn}, \mathrm{Co}, \mathrm{Cu}$ and $\mathrm{Zn}$ from inorganic sources (inorganic, $\mathrm{n}=11$ ) and additional $\mathrm{Mn}, \mathrm{Co}, \mathrm{Cu}$ and $\mathrm{Zn}$ from organic sources (organic, $\mathrm{n}=11$ ). Treatments were administered orally once per day via gelcap capsules using a balling gun. Animals in the control (basal) group received a cornmeal carrier devoid of $\mathrm{Mn}, \mathrm{Co}, \mathrm{Cu}$ and $\mathrm{Zn}$ and only obtained these trace minerals from dietary forages and concentrates in the basal diet. Cows assigned to trace mineral supplementation from inorganic sources were fed the basal diet supplemented with $200 \mathrm{mg} \mathrm{Mn,} 25 \mathrm{mg}$ Co, 125 $\mathrm{mg} \mathrm{Cu}$, and $360 \mathrm{mg} \mathrm{Zn} \mathrm{per} \mathrm{day} \mathrm{in} \mathrm{the} \mathrm{form} \mathrm{of} \mathrm{Mn} \mathrm{sulfate,} \mathrm{Co} \mathrm{carbonate,} \mathrm{Cu}$ sulfate, and $\mathrm{Zn}$ sulfate. Cows assigned to trace mineral supplementation from organic sources were fed the basal diet supplemented with the same amounts of trace minerals in the form of Mn-amino acid complex (Availa-Mn ${ }^{\circledR}$, Zinpro Corp., Eden Prairie, MN), Co glucoheptonate $\left(\mathrm{COPRO}^{\circledR}\right.$, Zinpro Corp.), Cu-amino acid complex (Availa-Cu ${ }^{\circledR}$, Zinpro Corp.), and Zn-amino acid complex (Availa-Mn ${ }^{\circledR}$, Zinpro Corp.). Cows on both the inorganic and organic treatments were further supplemented with these four trace minerals from inorganic sources added to each gelcap such that total daily supplementation was $65,2.5,15$, and $75 \mathrm{ppm}$ of the total daily DMI. This level of trace mineral supplementation for the animals in the inorganic and organic treatment groups met or exceeded NRC (2001) nutritional recommendations for dairy cattle. 
Table 5.1. Chemical composition of the basal prepartum and postpartum diets (DM basis)

\begin{tabular}{|c|c|c|c|}
\hline Nutrient & Far-off prepartum ${ }^{1,2,3}$ & Close-up prepartum & Postpartum \\
\hline$\overline{\text { Crude Protein, } \%}$ & 12.8 & 13.7 & 15.7 \\
\hline Available Protein, \% & 11.5 & 12.3 & 14.5 \\
\hline ADICP, \% & 1.3 & 1.4 & 1.1 \\
\hline Acid Detergent Fiber, $\%$ & 37.0 & 35.1 & 22.1 \\
\hline Neutral Detergent Fiber, \% & 55.3 & 51.2 & 31.6 \\
\hline Crude Fat, \% & 2.1 & 2.0 & 4.3 \\
\hline TDN, \% & 56.8 & 58.3 & 72.3 \\
\hline NEL, Mcal/kg & 1.3 & 1.3 & 1.7 \\
\hline NEm, Mcal/kg & 1.1 & 1.2 & 1.7 \\
\hline $\mathrm{NEg}, \mathrm{Mcal} / \mathrm{kg}$ & 0.6 & 0.6 & 1.1 \\
\hline Soluble Protein, \% CP & 22.2 & 23.8 & 37.5 \\
\hline Lignin, \% & 6.6 & 6.0 & 3.7 \\
\hline NDICP, \% & 5.2 & 4.9 & 3.4 \\
\hline Starch, \% & 5.8 & 10.1 & 29.2 \\
\hline $\mathrm{NFC}, \%$ & 26.0 & 29.0 & 44.5 \\
\hline Calcium, \% & 0.64 & 0.83 & 0.91 \\
\hline Phosphorus, \% & 0.21 & 0.23 & 0.27 \\
\hline Magnesium, \% & 0.36 & 0.40 & 0.35 \\
\hline Potassium, \% & 1.50 & 1.38 & 1.29 \\
\hline Sodium, \% & 0.11 & 0.12 & 0.30 \\
\hline Iron, ppm & 249.5 & 220.3 & 233.8 \\
\hline Zinc, ppm & 31.0 & 32.7 & 36.0 \\
\hline Copper, ppm & 11.0 & 10.8 & 9.8 \\
\hline Manganese, ppm & 81.8 & 72.0 & 35.8 \\
\hline Molybdenum, ppm & 1.4 & 1.3 & 1.6 \\
\hline Cobalt, ppm & 0.1 & 0.2 & 0.2 \\
\hline Sulfur, \% & 0.25 & 0.26 & 0.23 \\
\hline Ash, $\%$ & 8.9 & 9.0 & 7.3 \\
\hline
\end{tabular}

${ }^{\mathrm{T}}$ Values represent averages of samples composited every $4 \mathrm{wk}$.

${ }^{2}$ Basal diets were formulated to meet or exceed nutritional recommendations for all nutrients except $\mathrm{Mn}, \mathrm{Co}, \mathrm{Cu}$, and $\mathrm{Zn}$ (NRC, 2001).

${ }^{3}$ Cows received no dietary added (basal) or additional $200 \mathrm{mg}$ Mn, $25 \mathrm{mg}$ Co, $125 \mathrm{mg}$ $\mathrm{Cu}$, and $360 \mathrm{mg} \mathrm{Zn}$ per day in the form of $\mathrm{Mn}$ sulfate, Co carbonate, $\mathrm{Cu}$ sulfate, and $\mathrm{Zn}$ sulfate (inorganic) or Mn-amino acid complex, Co glucoheptonate, $\mathrm{Cu}$-amino acid complex, and Zn-amino acid complex (organic; Zinpro Corp.). The level of trace mineral supplementation from inorganic and organic sources met or exceeded NRC (2001) nutritional recommendations. 
Cows were housed in a free-stall barn, milked twice daily and fed individually for adlibitum feed intake via Calan doors (American Calan, Northwood, NH). Animals were enrolled on the study 65 days before expected parturition and removed from the experiment on day 8 after calving. A pre-treatment sample was collected on day $58.1 \pm$ 1.0 prior to calving and followed by initiation of dietary treatments within 3 days. Treatments were administered to cows until day 8 after parturition. To determine the effect of trace mineral supplementation from inorganic and organic sources on the function of circulating PMNL, approximately $450 \mathrm{~mL}$ of blood were collected on days $7.3 \pm 0.7$ before calving and $6.4 \pm 0.1$ after parturition. Blood was collected via jugular vein catheters into $500 \mathrm{~mL}$ conical tubes (Corning Inc., NY) containing $75 \mathrm{~mL}$ of acidcitrate dextrose, inverted three times and stored on ice until laboratory processing $(<20$ min). Catheters were inserted using aseptic techniques and immediately removed after collection of samples. All functional assays were performed simultaneously on the same day for any given cow.

\section{Reagents}

Percoll $^{\circledR}$ was obtained from GE Healthcare Bio-Sciences Corp. (Piscataway, NJ). Lipopolysaccharide from E. coli serotype O55:B5 (strain CDC 1644-70; conjugated to fluorescein isothiocyanate) was obtained from Sigma Chemical Co. (St. Louis, MO). Luminol (5-amino-2,3-dihydro-1,4-phthalazinedione; Sigma Chemical Co.) and phorbol 12-myristate,13-acetate (PMA; Sigma Chemical Co.) were prepared as $89.4 \mathrm{~m} M$ and 162.12 $\mu M$ stock solutions, respectively, in dimethyl sulfoxide (DMSO). Sytox ${ }^{\circledR}$ Orange nucleic acid stain was obtained from Invitrogen Co. (Carlsbad, CA) as a $5 \mathrm{~m} M$ solution in DMSO. Recombinant-human interleukin-8 (IL-8) was obtained from Sigma Chemical 
Co. The Vybrant ${ }^{\circledR}$ Phagocytosis Assay Kit was purchased from Invitrogen Co. All stock solutions were stored at $-20^{\circ} \mathrm{C}$. All working solutions, cell suspensions and reactions were prepared using endotoxin-free, calcium and magnesium-free Hanks' Balanced Salt Solution (CMF-HBSS; Sigma Chemical Co.) or RPMI-1640 (GIBCO, Grand Island, NY) and were pre-warmed to $37^{\circ} \mathrm{C}$ immediately prior to their use in the assays. Calciummagnesium free HBSS and RPMI-1640 were used in our in vitro cellular system to maintain a balanced environment (intra- and extracellular osmolarity, $\mathrm{pH},\left[\mathrm{O}_{2}\right]$, and temperature) and provide the cells with water and inorganic ions essential for normal cell metabolism. The use of HBSS as buffering medium during PMNL-chemiluminescence assays results in maximal light emission and cell activation compared to PBS and Tris (Freitas et al., 2009).

\section{Isolation of Bovine Neutrophils}

Neutrophils were isolated using procedures modified from Weber et al. (2001) as validated in our laboratory. Approximately $80 \mathrm{~mL}$ of blood were used to obtain PMNL for all functional assays. PMNL from the remaining blood were used to purify total mRNA for genetic expression profiling (reported elsewhere). Blood $(10 \mathrm{~mL})$ was aliquotted into 50-mL polypropylene conical tubes (Corning Inc.) and centrifuged at $1,000 \times g$ for $20 \mathrm{~min}$ at $4^{\circ} \mathrm{C}$ to separate plasma from the cell pack. Plasma was discarded and the remaining cells were suspended in $35 \mathrm{~mL}$ of CMF-HBSS. The suspension was gently pipetted down the side of a 50-mL polypropylene conical tube containing $10 \mathrm{~mL}$ of $1.084 \mathrm{~g} / \mathrm{mL}$ of Percoll ${ }^{\circledR}$. The cells were then subjected to gradient centrifugation (400 x $g$ for $40 \mathrm{~min}$ at $22^{\circ} \mathrm{C}$ ). The supernatant, mononuclear cell layer and Percoll ${ }^{\circledR}$ were aseptically aspirated and discarded. The pellet (PMNL and erythrocytes) was retained and 
erythrocytes were lysed by hypotonic shock. For this procedure, two volumes of an icecold $10.56 \mathrm{~m} M \mathrm{Na}_{2} \mathrm{HPO}_{4}, 2.67 \mathrm{~m} M \mathrm{NaH}_{2} \mathrm{PO}_{4}$ solution were added to one volume of cell suspension and the solution was mixed by gentle aspiration and inversion. Tonicity was restored by the addition of one volume of a $10.56 \mathrm{~m} M \mathrm{Na}_{2} \mathrm{HPO}_{4}, 2.67 \mathrm{~m} M \mathrm{NaH}_{2} \mathrm{PO}_{4} 0.43$ $M \mathrm{NaCl}$ solution. The cell solution was then centrifuged $(850 \times \mathrm{g})$ for $4 \min$ at $4^{\circ} \mathrm{C}$ and the pellet washed twice by resuspension in $35 \mathrm{~mL}$ of CMF-HBSS and recentrifugation (850 $\mathrm{x} g$ for $4 \mathrm{~min}$ at $4^{\circ} \mathrm{C}$ ). Cells were enumerated and viability was determined by propidium iodide (Sigma Chemical Co.) exclusion using a Cellometer Vision ${ }^{\circledR}$ automatic cell counter (Nexcelom Bioscience LLC., Lawrence, MA).

\section{LPS Incubation}

A working solution of $500 \mu \mathrm{g} / \mathrm{mL}$ LPS was added to $50-\mathrm{mL}$ polypropylene conical tubes (Corning Inc.) containing purified neutrophils to reach final concentrations of 0 or $50 \mu \mathrm{g} / \mathrm{mL}$ of Escherichia coli LPS at a cell concentration of $4 \times 10^{6}$ cells $/ \mathrm{mL}$. The tubes were then placed in a humidified incubator $\left(37^{\circ} \mathrm{C}, 5 \% \mathrm{CO}_{2}\right)$ and incubated for 120 minutes while inverted in a Labquake ${ }^{\circledR}$ tube rotator. After incubation, the cells solutions were centrifuged $(850 \times g)$ for $4 \mathrm{~min}$ at $4^{\circ} \mathrm{C}$, the supernatant was discarded and the pellet resuspended in CMF-HBSS. Cells were enumerated and viability was assessed by propidium iodide exclusion using a Cellometer Vision ${ }^{\circledR}$ automatic cell counter (Nexcelom Bioscience LLC.).

\section{Luminol Chemiluminescence Assay}

After incubation with LPS, an index of the total neutrophil ROS production was immediately assessed by a luminol chemiluminescence assay as described by Revelo and Waldron (2010). Fifty microliters of cell suspension were pipetted into the wells $(2.5 \mathrm{x}$ 
$10^{5}$ cells/well) of white-walled, clear-bottomed 96-well microplates (Corning Inc.). All reactions were carried out in sixtuplicates. Twenty $\mu \mathrm{L}$ of $5 \mathrm{~m} M$ 5-amino-2,3-dihydro-1,4phthalazinedione (luminol) and $20 \mu \mathrm{L}$ of either $400 \mathrm{n} M$ PMA (stimulated cells) or HBSS (non-stimulated cells) were added to all wells. All reactions were then adjusted to $200 \mathrm{uL}$ using CMF-HBSS. Chemiluminescence (CL) was measured every $5 \mathrm{~min}$ for $240 \mathrm{~min}$ with a Synergy HT plate reader (BioTek Instruments, Inc., Winooski, VT). Background values, defined as the mean CL values of luminol diluted in CMF-HBSS, were automatically subtracted from all readings. Individual curves were generated from plotted CL values over time for each experimental condition. The area under the curve (AUC), maximal CL, and time to maximal CL were obtained from plotted CL values.

\section{Neutrophil Extracellular Trap (NET) Assay}

Neutrophil extracellular trap formation was quantified using Sytox ${ }^{\circledR}$ Orange as described by Revelo and Waldron (2010). After PMNL incubation with LPS, $100 \mu \mathrm{L}$ of cell suspension were pipetted into the wells $\left(6.25 \times 10^{5}\right.$ cells/well) of white-walled, clearbottomed 96-well microplates (Costar Inc., NY). All reactions were performed in sixtuplicates. Twenty microliters of PMA and $20 \mathrm{uL}$ of ionomycin to obtain a final concentration of $10 \mathrm{ng} / \mathrm{ml}$ and $1 \mu M$, respectively, were added to the stimulated set of wells. Forty microliters of RPMI-1640 $+2 \%$ heat-inactivated FBS were added to the control wells. The plates were then centrifuged $(10 \mathrm{~min}$ at $650 \times g)$ and incubated $\left(37^{\circ} \mathrm{C}\right.$ and $5 \% \mathrm{CO}_{2}$ ) for $60 \mathrm{~min}$. After incubation, supernatant from the plates was removed, and the wells were stained and protected from light with $50 \mu$ of $5 \mu M$ Sytox Orange in CMF-HBSS (15 min at room temperature). The stain was poured off, and the wells washed three times with $100 \mu \mathrm{l}$ of CMF-HBSS. A Synergy HT plate reader was used to 
detect fluorescence with an excitation wavelength of $530 \mathrm{~nm}$ and emission wavelength of $580 \mathrm{~nm}$. Background values, defined as the mean fluorescence values of PMA and ionomycin diluted in RPMI-1640 were subtracted from all readings.

\section{Chemotaxis Assay}

Chemotaxis was performed in a 48-well micro chemotaxis chamber (Neuro Probe Inc., Gaithersburg, MD) according to the manufacturer's recommendations. The wells in the bottom plate of the chamber were filled with $26 \mu \mathrm{L}$ of the chemoattractant interleukin 8 (IL-8; $100 \mathrm{ng} / \mathrm{mL}$, Sigma Chemical Co.) or CMF-HBSS, previously warmed to $37^{\circ} \mathrm{C}$. A slight positive meniscus was formed when the wells were filled with the chemoattractant. A $25 \times 80 \mathrm{~mm}$ polycarbonate filter (non-polyvinylpyrrolidone treated, Neuro Probe Inc.) was lowered onto the formed meniscus, allowing the middle portion of the filter to make contact first. The polycarbonate filter had pores with a diameter of $5 \mu \mathrm{m}$ to allow migration and adhesion of PMNL to the underside of the filter where they could be stained and counted. A silicone gasket was then applied on top of the filter and the top component of the micro chemotaxis chamber fastened to the bottom chamber. The chamber was pre-warmed for $10 \mathrm{~min}$ at $37^{\circ} \mathrm{C}$ before adding the PMNL to the upper chamber. Fifty-five $\mu 1$ of cell suspensions $\left(3.75 \times 10^{6}\right.$ neutrophils $\left./ \mathrm{ml}\right)$ previously incubated with 0 or $50 \mu \mathrm{g} / \mathrm{mL}$ of $E$. coli LPS were added to the top chamber and incubated at $37^{\circ} \mathrm{C}$ in humidified air with $5 \% \mathrm{CO}_{2}$ for $30 \mathrm{~min}$ to allow the PMNL to migrate towards the chemoattractant. After the incubation, the fluid from the top chamber was aspirated and the chamber disassembled. The side of the filter membrane that had faced the upper chamber was rinsed with CMF-HBSS and wiped off three times with a wiper blade to remove non-migrated PMNL. Migrated PMNL were adhered to the side of 
the filter that had faced the IL-8 (chemoattractant) or CMF-HBSS (control). The filter was immersed in methanol for $10 \mathrm{~min}$, air-dried and stained in a 3.75\% Giemsa staining solution (Sigma Chemical Co.) for $1 \mathrm{hr}$. Subsequently, the membrane was rinsed in Giemsa buffer and placed with the cell-side up on a 50 × $75 \mathrm{~mm}$ microscope slide for drying. The number of PMNL that migrated through the filter was counted under oil immersion on a light microscope by counting the number of neutrophils present in 7 microscope fields at 1,000 x. Each sample was run in triplicate. The average number of PMNL that migrated towards CMF-HBSS (random migration) was subtracted from the average number of PMNL that migrated towards IL-8 (directed migration). Results are reported as the average number of migrated neutrophils towards IL-8 observed per seven fields.

\section{Phagocytosis Assay}

Neutrophil phagocytosis of fluorescent Escherichia coli (K-12 strain) Bioparticles $^{\circledR}$ was determined using the Vybrant $^{\circledR}$ Phagocytosis Assay Kit (Invitrogen Co.) following manufacturer's recommendations. The E. coli Bioparticles ${ }^{\circledR}$ powder was diluted in $5 \mathrm{~mL}$ of HBSS containing $2 \mathrm{mM}$ of sodium azide, briefly sonicated with a sonic dismembrator (Sonifier ${ }^{\circledR}$, Branson Ultrasonics Corp.). This suspension was supplemented with adult bovine serum to reach a final concentration of $10 \%$ and incubated on a Labquake ${ }^{\circledR}$ tube rotator for $30 \mathrm{~min}$ at $37^{\circ} \mathrm{C}$. One hundred $\mu \mathrm{l}$ of the fluorescein-labeled E. coli Bioparticles ${ }^{\circledR}$ suspension or HBSS (control) were added to the wells of white-walled, v-bottom 96-well microplates (Costar Inc., NY) containing 2.5 x $10^{6}$ PMNL. All reactions were carried out in singlicate. After a 60-min incubation at $37^{\circ} \mathrm{C}$ in an orbital shaker at $150 \mathrm{rpm}$, the microplates were centrifuged $\left(4^{\circ} \mathrm{C}, 3 \mathrm{~min}\right.$ at 500 
$\times \mathrm{g}$ ) and the supernatant discarded. To quench non-phagocytized E. coli particles, $100 \mu \mathrm{L}$ of a trypan blue solution $(0.25 \mathrm{mg} / \mathrm{mL})$ was added to the wells containing PMNL with phagocytized E. coli bioparticles and the microplate was incubated for 2 min. The microplates were then centrifuged again $\left(4^{\circ} \mathrm{C}, 3 \mathrm{~min}\right.$ at $\left.500 \times \mathrm{g}\right)$ and the supernatant discarded. The pelleted PMNL were then fixed with $200 \mu \mathrm{L}$ of a $2 \%$ formaldehyde solution and stored protected from light at $4^{\circ} \mathrm{C}$ until flow cytometry analysis. Flow cytometric analyses were performed with a CyAn ADP flow cytometer (Beckman Coulter Inc., Miami, FL). Approximately 20,000 viable PMNL were counted in the gate according to side and scatter characteristics. The population of PMNL positive for fluorescent phagocytized E. coli Bioparticles ${ }^{\circledR}$ was then separated from PMNL containing no intracellular E. coli particles. The percentage of fluorescence-positive PMNL and their mean fluorescence intensity (MFI) were determined using Summit Sofware v4.3 (Beckman Coulter Inc.). Background fluorescence values obtained from reactions containing only PMNL (control wells) were subtracted from the MFI values for fluorescence-positive PMNL.

\section{Killing Assay}

The antimicrobial ability of PMNL against Escherichia coli was measured using a fluorescence-based microplate assay modified from Shiloh et al. (1997). E. coli P4:O37 (kindly provided by Dr. Joe Hogan, The Ohio State University) was inoculated on a blood agar plate (Northeast Laboratory Services Inc., ME) and incubated overnight at $37^{\circ} \mathrm{C}$. Ten colonies were transferred from the plate to a sterile tube containing $10 \mathrm{~mL}$ of brain-heart infusion broth (Sigma Chemical Co.) and incubated overnight at $37^{\circ} \mathrm{C}$ at 225 rpm in an orbital shaker. After the overnight incubation, the tube was sonicated to 
disassociate individual bacterial cells using a sonic dismembrator $\left(\right.$ Sonifier ${ }^{\circledR}$, Branson Ultrasonics Corp., Danbury, CT) and maintained at $4^{\circ} \mathrm{C}$ to stop bacterial growth. One hundred microliters from this culture were serially diluted in CMF-HBSS and $100 \mu \mathrm{L}$ of the resulting dilutions were spread on blood agar plates. The stock culture was maintained at $4{ }^{\circ} \mathrm{C}$ and the plates were incubated overnight at $37^{\circ} \mathrm{C}$ to determine the concentration [colony forming units $(\mathrm{CFU}) / \mathrm{mL}$ ] of the stock culture based on the colony counts of the spread plates. Immediately before the initiation of the killing assay, the stock culture was placed in ice and diluted in HBSS to yield a final concentration of $1.6 \mathrm{x}$ $10^{8} \mathrm{cfu} / \mathrm{mL}$. To assess PMNL killing, $200 \mu \mathrm{L}$ of E. coli $\left(3.2 \times 10^{7} \mathrm{cfu}\right)$ and $400 \mu \mathrm{L}$ of pooled, heat-inactivated bovine serum were added to sterile tubes with or without $2 \times 10^{6}$ PMNL previously incubated with 0 or $50 \mu \mathrm{g} / \mathrm{mL}$ of LPS. The ratio of bacteria to PMNL was 16:1. All reactions were performed in triplicate. The samples were placed on a Labquake ${ }^{\circledR}$ tube rotator for $60 \mathrm{~min}$ at $37^{\circ} \mathrm{C}$. These reactions were then placed in ice and sonicated with a sonic dismembrator for $15 \mathrm{~s}$ at amplitude of $20 \%$. Rupture of the PMNL was verified by microscopic examination. Sonication does not affect bacterial viability (Rinaldi et al., 2007; Revelo and Waldron, unpublished observation).

To determine the killing capacity of PMNL against E. coli, a $75 \mu \mathrm{L}$ aliquot from each sonicated reaction was pipetted in duplicates into the wells of white-walled, clearbottomed 96-well microplates (Corning Inc.) containing $150 \mu \mathrm{L}$ of brain-heart infusion broth medium supplemented to $15 \%$ AlarmarBlue ${ }^{\circledR}$ (Invitrogen Co.). In addition, $225 \mu \mathrm{L}$ of $15 \%$ medium-AlarmarBlue were plated in triplicates to serve as the reagent blank. The plate was immediately placed in a Synergy HT plate reader at $37^{\circ} \mathrm{C}$ and the fluorescence recorded every ten minutes for $14 \mathrm{hr}$. with an excitation wavelength of $530 \mathrm{~nm}$ and 
emission wavelength of $590 \mathrm{~nm}$. As bacteria grow, they convert AlarmarBlue ${ }^{\circledR}$ from its non-fluorescent oxidized to its fluorescent oxidized state (Shiloh et al., 1997). Fluorescence values in arbitrary units from each well were plotted over time after subtraction of the fluorescence values from wells with the reagent blank. For each curve, a line was constructed using the data points that best fitted a linear curve $(y=a \mathrm{x}+b)$. The time to half-maximal fluorescence (thmax) was calculated by dividing the difference between half-maximal fluorescence (hmax) and the intercept $(b)$ by the slope $(a)$. The time required to achieve hmax is inversely proportional to the number of viable bacteria present when AlarmarBlue ${ }^{\circledR}$ is added (Shiloh et al., 1997). This relationship was used to estimate the number of bacteria present in the reactions with or without PMNL based on thmax values. The number of viable bacteria was calculated using a standard curve for $E$. coli concentration based on AlarmarBlue ${ }^{\circledR}$ thmax values. To generate this standard curve, serial 10-fold dilutions of a stock culture were prepared and aliquots ( $75 \mu \mathrm{L})$ from each dilution were plated in triplicate into the wells of a microplate containing $150 \mu \mathrm{L}$ of $15 \%$ medium-AlarmarBlue ${ }^{\circledR}$. The thmax for each dilution was determined as described above. Aliquots $(75 \mu \mathrm{L})$ from each dilution were spread on blood agar plates. The plates were incubated overnight at $37^{\circ} \mathrm{C}$ and the colonies forming units (CFU) enumerated. The calculated thmax value for each dilution was plotted against CFU in the logarithmic scale (base 10) and the linear function $y=-0.0186 x+16.854, r^{2}>0.99$ (where $y=\log$ CFU and $\mathrm{x}=$ thmax) was generated from the plotted data points. This function was then used to calculate the number of viable CFU from thmax values obtained in all experiments. The percentage of bacteria killed were determined by calculating the difference in the 
number of bacteria incubated in the absence and presence of PMNL and dividing this difference by the number of bacteria incubated in the absence of PMNL.

\section{Statistical Analyses}

This study was conducted as a randomized complete block design with cow as the experimental unit. Cows were randomly assigned to one of 13 blocks according to their BCS, SCC and previous lactation ME 305 milk production. Cows within each block were assigned to one of the three dietary treatments. Further, PMNL collected from each cow were randomly assigned to in vitro incubations with either 0 or $50 \mu \mathrm{g} / \mathrm{mL}$ of $E$. coli LPS. Areas under the curves (AUC) were calculated from plotted data points for each experimental condition using the expand procedure of SAS with cubic spline interpolation and the trapezoid rule (SAS, 2001). Extreme observations were detected using PROC REG of SAS (2001) and removed from the data set if they presented at least 3 of the following characteristics: standardized residual $\left(\mathrm{r}_{\mathrm{i}}\right)>2$ standard deviations, high leverage $\left(h_{i i} \geq 2 \mathrm{pn}^{-1}\right.$, where $\mathrm{n}=$ number of observations and $\mathrm{p}=$ number of parameters $)$, possible undue influence $\left[\right.$DFITTS $_{\mathrm{i}} \geq 2\left({ } \mathrm{pn}^{-1}\right)^{-1}$, DFBETAS $\left._{\mathrm{i}} \geq 2(\sqrt{ } \mathrm{n})^{-1}\right]$, cook's distance $\left(\mathrm{D}_{\mathrm{i}}\right)>1$ and ratio of generalized variances $\left(\mathrm{COVRATIO}_{\mathrm{i}}\right)<1-3 \mathrm{pn}^{-1}$ or $>1+3 \mathrm{pn}^{-1}$ (Kaps and Lamberson, 2004). The distribution of all variables was examined using the Capability procedure of SAS (2001). For the variables that followed a normal distribution, data were analyzed by analysis of variance (ANOVA) as a mixed model using the mixed procedure of SAS (2001). For the variables that failed the goodness-offit tests for normality $(P<0.05)$, data were analyzed by ANOVA as a generalized linear mixed model implemented in PROC GLIMMIX of SAS (2001) which allows for nonnormally distributed data. The independent variables in the model statement included 
dietary supplementation, LPS treatment, stage of lactation and their interaction. The random statement defined block and the interaction of block by dietary supplementation interaction as random effects. The repeated statement was used to specify a repeated measurement model with period as the repeated effect, cow as the subject and a compound symmetry covariance structure (Littell et al., 1998). Analysis of covariance (ANCOVA) was conducted to assess the potential influence of the pre-treatment measurements collected on day 58 prepartum on the outcome variables. This analysis of covariance was implemented by defining the pre-treatment value as a continuous variable in the MODEL statement but not in the CLASS statement. Pre-treatment measurements were not included in the dataset during ANCOVA. Non-significant covariates $(P>0.05)$ were dropped from the MODEL statement. Separate ANOVA were conducted to determine the main effects of day relative to calving and LPS on the dependent variables. These analyses included the pre-treatment measurements in the dataset. For those variables analyzed by ANOVA as a generalized linear mixed model (PROC GLIMMIX), the type of distribution was specified in the model statement using the DIST and LINK functions of SAS. Least squares means and standard errors were generated using the LSMeans statement in conjunction with the pdiff option of SAS. Tukey's multiple comparison test was used only to assess specific treatment differences for those variables shown to have significant overall treatment effects in the analysis of variance. For normally distributed variables, the reported means are the adjusted least squares means \pm SEM. For variables with a lognormal distribution, the least squares means in the logarithmic scale generated by the generalized linear mixed model (PROC GLIMMIX) were back-transformed by raising 10 to the power of the values. To avoid the bias 
generated when back-transforming SEM, confidence intervals (CI) were produced using back-transformed confidence limits. Therefore, the reported means are back-transformed adjusted least squares means \pm CI. Significance was declared when $P<0.05$.

\section{RESULTS}

\section{Reactive Oxygen Species Production}

Luminol-derived CL, an index of the overall generation of ROS by PMNL, was measured every $5 \mathrm{~min}$ for a period of $240 \mathrm{~min}$ after stimulation of PMNL with PMA (data not shown). These CL values were plotted over time and used to calculate the AUC representing the overall amount of ROS produced by the stimulated PMNL (Figures 5.1A, 1B, and 1C), the maximal luminol-derived ROS production (Figures 5.1D, 5.1E, and 5.1F), and the time required for the PMNL to reach maximal ROS generation (Figures 5.1G, 5.1H, and 5.1I). There was no effect of interaction of trace mineral supplementation source by period on the total amount of ROS produced (Figure 5.1A, $P$ $=0.126$ ) and maximal ROS generation (Figure 5.1D, $P=0.114$ ). Although an effect of interaction of trace mineral source by period on the time to reach maximal ROS was detected (Figure 5.1G, $P=0.032$ ), the mean time to maximal ROS did not differ between treatment groups within each day relative to calving $(\mathrm{P}>0.050)$. There was no main effect of supplementation source on any of the parameters of ROS generation measured in this study (data not shown, $P>0.050$ ). However, the total ROS production by PMNL was $15 \%$ lower on day 7 prior to parturition $(P=0.007)$ and $38 \%$ decreased on day 6 after parturition $(P<0.001)$, compared with PMNL collected from cows 58 days before calving (Figure 5.1B; main effect of period, $P<0.001$ ). Maximal ROS generation was 
not different between days 58 and 7 prepartum $(P=0.638)$, but decreased on day 6 postpartum by 37 and $40 \%$, compared with days $7(P<0.001)$ and $58(P<0.001)$ prepartum, respectively (Figure 5.1E; main effect of period, $P<0.001$ ). In agreement, PMA-stimulated PMNL collected from cows on day 58 prepartum reached maximal ROS generation 23 and 40 min earlier than PMNL collected from cows on days 7 prepartum $(P=0.033)$ and 6 postpartum $(P<0.001)$, respectively. The time to reach PMNL maximal ROS production was not different $(P=0.202)$ between days 7 prepartum and 6 postpartum (Figure 5.1H; main effect of period, $P<0.001$ ). Incubation of PMNL with 50 $\mu \mathrm{g} / \mathrm{mL}$ of LPS had no effect on PMNL production of ROS (Figure 5.1C, $P=0.393$ ) and maximal generation of ROS (Figure 5.1F, $P=0.077$ ). In contrast, PMNL incubated with $50 \mu \mathrm{g} / \mathrm{mL}$ of LPS reached maximal ROS production 63 min earlier than control PMNL $(P>0.001)$ after cell stimulation with PMA (Figure 5.1I; main effect of LPS, $P<0.001$ ). 
Figure 5.1. Effect of trace mineral supplementation source on an index of the total reactive oxygen species (ROS) production by bovine neutrophils (PMNL). Cows were fed basal diets with no added dietary $\mathrm{Mn}, \mathrm{Co}, \mathrm{Cu}$ and $\mathrm{Zn}$ (basal, $\mathrm{n}=13$ ) or supplemented with $200 \mathrm{mg} \mathrm{Mn}, 25 \mathrm{mg} \mathrm{Co}, 125 \mathrm{mg} \mathrm{Cu}$, and $360 \mathrm{mg} \mathrm{Zn}$ from either inorganic (inorganic, $\mathrm{n}=11$ ) or organic sources (organic, $\mathrm{n}=11$ ). Treatments were administered to the cows between days $57.14 \pm 1.0$ prior to parturition until day 8 after parturition. PMNL were collected from the blood of cows on days 58.14 \pm 1.0 (pre-treatment sample), $7.30 \pm 0.7$ prior to parturition and $6.37 \pm 0.1$ after parturition. Subsequently, the PMNL were incubated with 0 or $50 \mu \mathrm{g} / \mathrm{mL}$ of Escherichia coli lipopolysaccharide (LPS) for $120 \mathrm{~min}$. The luminol-derived chemiluminescence (CL) was measured every $5 \mathrm{~min}$ over a 240 min period after addition of phorbol 12-myristate,13-acetate (PMA; $400 \mathrm{nM}$ ). CL values of wells without PMNL (containing HBSS and reagents only) were subtracted from CL measurements at every time point. Areas under the curves (AUC; panels A, B, and $\mathrm{C}$ ), maximal luminol-dependent ROS production (arbitrary units; panels D, E, and F), and time to maximal ROS production (panels $\mathrm{G}, \mathrm{H}$, and I) were calculated from plotted luminol-derived CL measurements. (A) Effect of interaction of trace mineral supplementation source by period on the overall production of ROS (AUC) by PMAstimulated PMNL, $P=0.126$. (B) Main effect of period on the overall production of ROS (AUC) by PMA-stimulated PMNL, $P<0.001$. (C) Main effect of LPS on the overall production of ROS (AUC) by PMA-stimulated PMNL, $P=0.393$. (D) Effect of interaction of trace mineral supplementation source by period on the maximal luminoldependent ROS production (arbitrary units) by PMA-stimulated PMNL, $P=0.114$. (E) Main effect of period on the on the maximal luminol-dependent ROS production (arbitrary units) by PMA-stimulated PMNL, $P<0.001$. (F) Main effect of LPS on the on the maximal luminol-dependent ROS production (arbitrary units) by PMA-stimulated PMNL, $P=0.077$. (G) Effect of interaction of trace mineral supplementation source by period on the time to maximal ROS production (min) by PMA-stimulated PMNL, $P=$ 0.032. (H) Main effect of period on the on the time to maximal ROS production (min) by PMA-stimulated PMNL, $P<0.001$. (I) Main effect of LPS on the time to maximal ROS production ( $\mathrm{min}$ ) by PMA-stimulated PMNL, $P<0.001$. Bars represent the adjusted least square means and the error bars indicate the standard error of the means. ${ }^{a, b}$ Bars with different letters differ at $P<0.05$ (Tukey). 

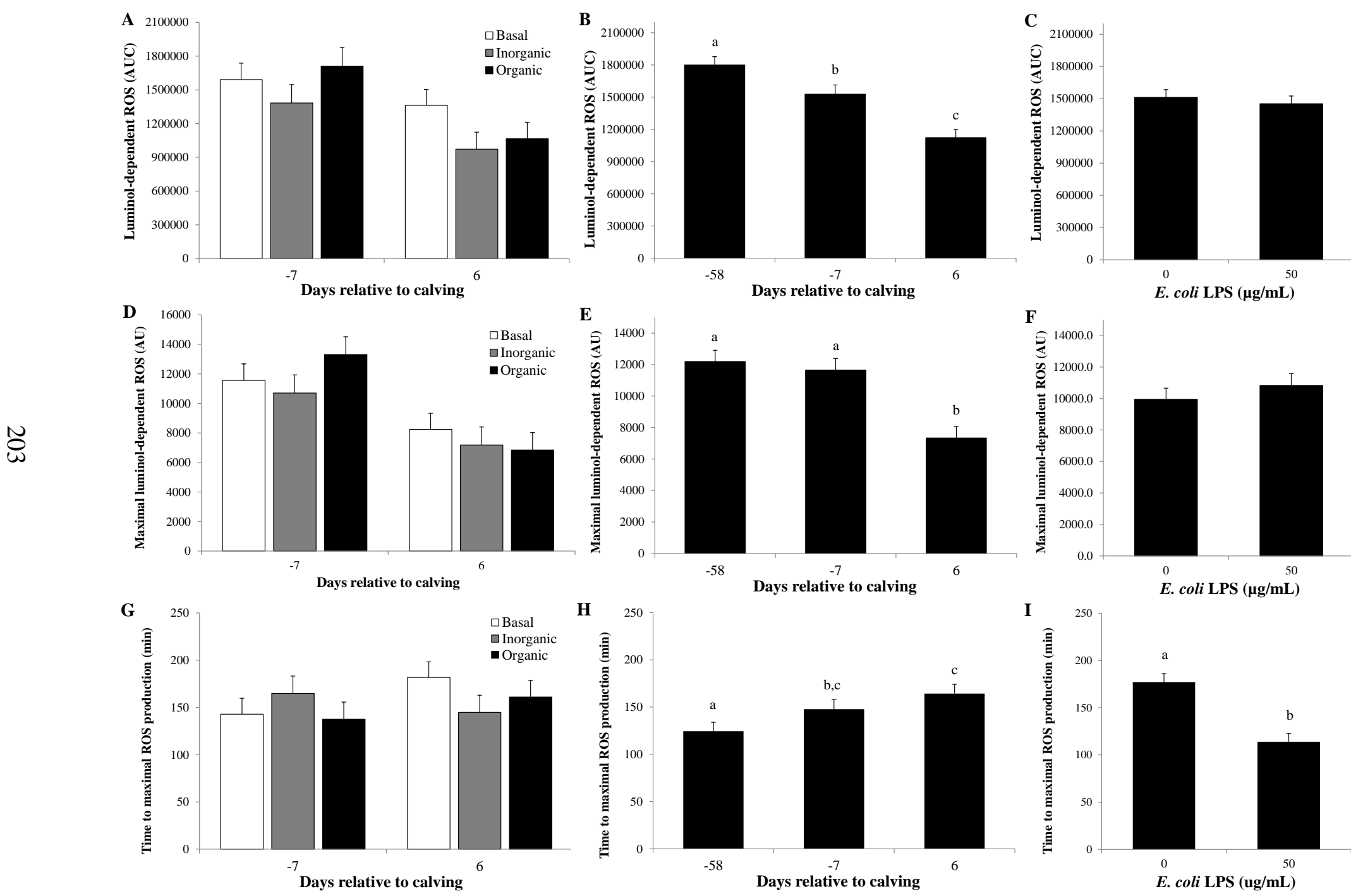


\section{Neutrophil Extracellular Trap Formation}

The release of NETs was quantified in PMA-stimulated (Figures 5.2A, 5.2B, and 5.2C) and non-stimulated (Figures 5.2D, 5.2E, and 5.2F) PMNL. There was no effect of interaction of trace mineral supplementation source by period on NET release by PMAstimulated (Figure 5.2A, $P=0.073$ ) and non-stimulated PMNL (Figure 5.2D, $P=0.156$ ). Similarly, there was no main effect of supplementation source on NET release by PMAstimulated and non-stimulated PMNL (data not shown, $P>0.050$ ). Independently of trace mineral supplementation, the release of NETs by PMA-stimulated PMNL was 27 and $36 \%$ lower $(P<0.001)$ in cows on day 58 prepartum, compared with day 7 prepartum and 6 postpartum, respectively. NET release by PMA-stimulated PMNL was $13 \%$ lower $(P=0.003)$ in PMNL from cows on day 7 prepartum, compared to day 7 postpartum (Figure 5.2B, main effect of period, $P<0.001$ ). In non-stimulated PMNL, the release of NETs was 28 and 78\% lower $(P<0.001)$ in PMNL collected from cows on day 58 prepartum, compared with day 7 prepartum and 6 postpartum, respectively. Release of NETs by non-stimulated PMNL was $22 \%$ lower $(P=0.022)$ in PMNL from cows on day 7 prepartum, compared to day 6 postpartum (Figure 5.2E, main effect of period, $P<$

0.001). Incubation of PMNL with $50 \mu \mathrm{g} / \mathrm{mL}$ of LPS had no effect on NET generation by PMA-stimulated PMNL (Figure 5.2C, $P=0.080$ ). In contrast, non-stimulated PMNL incubated with $50 \mu \mathrm{g} / \mathrm{mL}$ of LPS had $46 \%$ higher $(P>0.001)$ NET release than control PMNL (Figure 5.2F; main effect of LPS, $P<0.001$ ). 
Figure 5.2. Effect of trace mineral supplementation source on Neutrophil Extracellular Trap release (NET) by bovine neutrophils (PMNL). Cows were fed basal diets with no added dietary $\mathrm{Mn}, \mathrm{Co}, \mathrm{Cu}$ and $\mathrm{Zn}$ (basal, $\mathrm{n}=13$ ) or supplemented with 200 $\mathrm{mg} \mathrm{Mn}, 25 \mathrm{mg} \mathrm{Co}, 125 \mathrm{mg} \mathrm{Cu}$, and $360 \mathrm{mg} \mathrm{Zn}$ from either inorganic (inorganic, $\mathrm{n}=11$ ) or organic sources (organic, $\mathrm{n}=11$ ). Treatments were administered to the cows between days $57.14 \pm 1.0$ prior to parturition until day 8 after parturition. PMNL were collected from the blood of cows on days $58.14 \pm 1.0$ (pre-treatment sample), $7.30 \pm 0.7$ prior to parturition and $6.37 \pm 0.1$ after parturition. Subsequently, the PMNL were incubated with 0 or $50 \mu \mathrm{g} / \mathrm{mL}$ of Escherichia coli lipopolysaccharide (LPS) for $120 \mathrm{~min}$. Release of NETs was assessed by measuring the fluorescence of cells stained with Sytox Orange ${ }^{\circledR}$ (Invitrogen Co., Eugene, OR) after a 1-h incubation with $16.21 \mathrm{nM}$ (stimulated PMNL; panels A, B, and C) or $0 \mathrm{nM}$ of phorbol 12-myristate,13-acetate (PMA; non-stimulated PMNL; panels D, E, and F). (A) Effect of interaction of trace mineral supplementation source by period on NET release by PMA-stimulated PMNL, $P=0.073$. (B) Main effect of period on NET release by PMA-stimulated PMNL, $P<0.001$. (C) Main effect of LPS on NET release by PMA-stimulated PMNL, $P=0.080$. (D) Effect of interaction of trace mineral supplementation source by period on NET release by non-stimulated PMNL, $P=$ 0.156. (E) Main effect of period on NET release by non-stimulated PMNL, $P<0.001$. (F) Main effect of LPS on NET release by non-stimulated PMNL, $P<0.001$. Bars in panels $\mathrm{A}, \mathrm{B}$, and $\mathrm{C}$ represent the adjusted least square means and the error bars indicate the standard error of the means. Bars in panels D, E, and F represent adjusted least square means back-transformed from the logarithmic scale and the error bars indicate confidence intervals. ${ }^{\mathrm{a}, \mathrm{b}}$ Bars with different letters differ at $P<0.05$ (Tukey). 

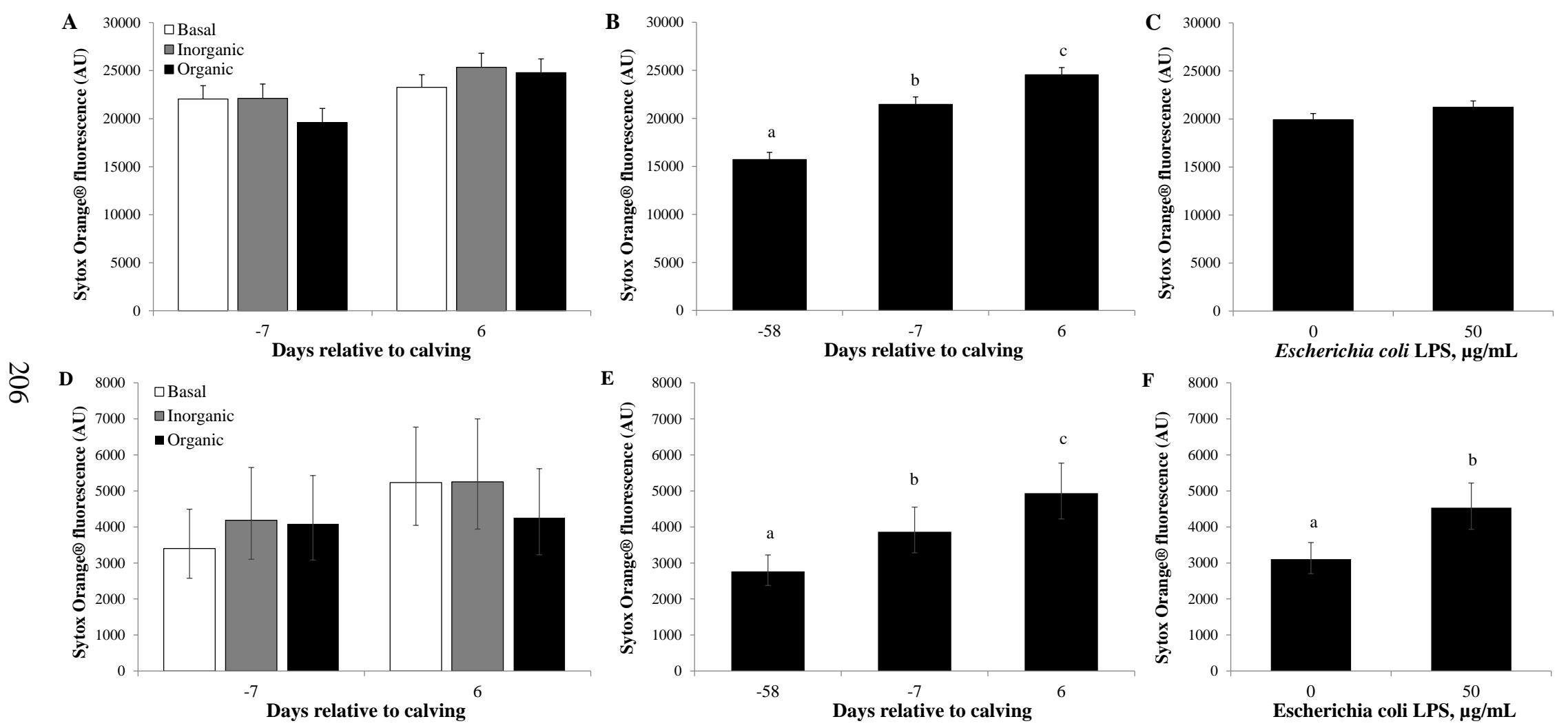


\section{Chemotaxis}

There was no effect of interaction of trace mineral supplementation source by period on PMNL chemotaxis towards IL-8 (Figure 5.3A, $P=0.325$ ). Similarly, there was no main effect of trace mineral supplementation source on chemotaxis of PMNL towards IL-8 (data not shown, $P=0.781$ ). Neutrophil chemotaxis decreased by $18 \%$ between days 58 prepartum and 6 postpartum $(P=0.018)$, but did not differ $(P>0.200)$ between days 58 and 7 prepartum or 7 prepartum and 6 postpartum (Figure 5.3B; main effect of period, $P=0.024)$. Incubation of PMNL with $50 \mu \mathrm{g} / \mathrm{mL}$ of LPS had no effect on chemotaxis of PMNL towards IL-8 (Figure 5.3C, $P=0.832$ ). 
Figure 5.3. Effect of trace mineral supplementation source on neutrophil (PMNL) chemotaxis towards the chemoattractant interleukin-8 (IL-8). Cows were fed basal diets with no added dietary $\mathrm{Mn}, \mathrm{Co}, \mathrm{Cu}$ and $\mathrm{Zn}$ (basal, $\mathrm{n}=13$ ) or supplemented with $200 \mathrm{mg}$ $\mathrm{Mn}, 25 \mathrm{mg} \mathrm{Co}, 125 \mathrm{mg} \mathrm{Cu}$, and $360 \mathrm{mg} \mathrm{Zn}$ from either inorganic (inorganic, $\mathrm{n}=11$ ) or organic sources (organic, $\mathrm{n}=11$ ). Treatments were administered to the cows between days $57.14 \pm 1.0$ prior to parturition until day 8 after parturition. PMNL were collected from the blood of cows on days $58.14 \pm 1.0$ (pre-treatment sample), $7.30 \pm 0.7$ prior to parturition and $6.37 \pm 0.1$ after parturition. Subsequently, the PMNL were incubated with 0 or $50 \mu \mathrm{g} / \mathrm{mL}$ of Escherichia coli lipopolysaccharide (LPS) for $120 \mathrm{~min}$. Chemotaxis was determined as the mean number of migrated PMNL towards IL-8 observed in seven fields at 1000x. (A) Effect of interaction of trace mineral supplementation source by period on chemotaxis of PMNL towards IL-8, $P=0.325$. (B) Main effect of period on chemotaxis of PMNL towards IL-8, $P=0.024$. (C) Main effect of LPS on chemotaxis of PMNL towards IL- $8, P=0.832$. Bars represent the adjusted least square means and the error bars indicate the standard error of the means. ${ }^{\mathrm{a}, \mathrm{b}} \mathrm{B}$ ars with different letters differ at $P$ $<0.05$ (Tukey). 

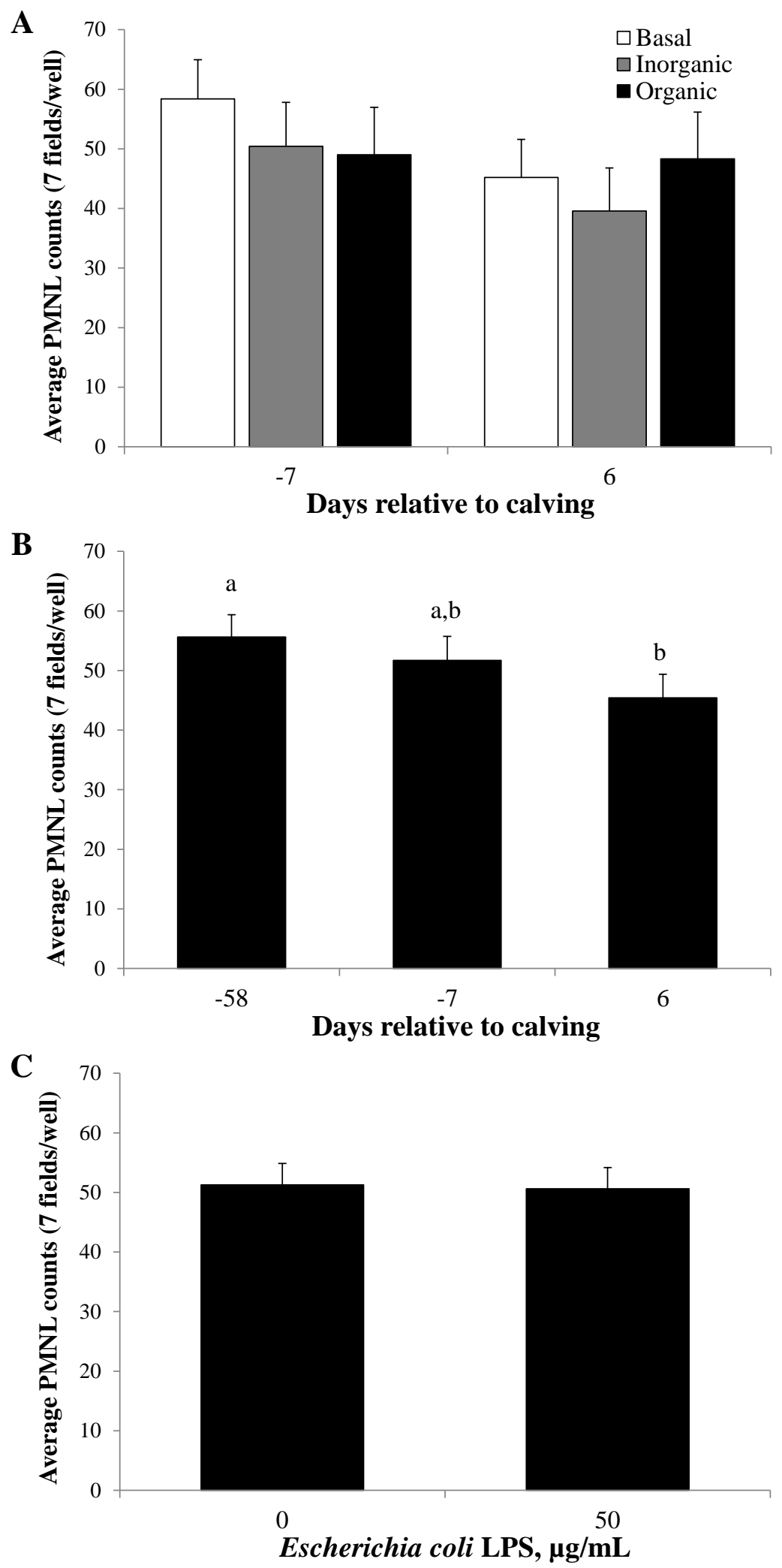


\section{Phagocytosis}

Phagocytosis of E. coli by PMNL was assessed as the percentage of PMNL positive for phagocytized E. coli particles (Figures 5.4A, 5.4B, and 5.4C) and their mean fluorescence intensity (Figures 5.4D, 5.4E, and 5.4F). There was no effect of interaction of trace mineral supplementation source by period on the percentage of PMNL positive for phagocytized $E$. coli particles (Figure 5.4A, $P=0.098$ ). Also, no main effect of trace mineral supplementation source on PMNL positive for phagocytized E. coli particles (data not shown, $P=0.749$ ). The percentage of PMNL with phagocytized $E$. coli particles was 44 and $26 \%$ lower on days 7 prepartum and 6 postpartum $(P<0.001)$, compared with day 58 prepartum, respectively. However, the percentage of PMNL positive for phagocytized $E$. coli was $31 \%$ higher $(P<0.001)$ on day 6 postpartum, compared with day 7 prepartum (Figure 5.4B; main effect of period, $P<0.001$ ). Independently of trace mineral source, incubation of PMNL with $50 \mu \mathrm{g} / \mathrm{mL}$ of LPS had no effect on the percentage of PMNL positive for phagocytized $E$. coli (Figure $5.4 \mathrm{C}, P=0.580$ ). An effect of interaction of trace mineral supplementation source by period on the mean fluorescence intensity of phagocytic PMNL was detected (Figure 5.4D, $P=0.007$ ). On day 7 prepartum, no differences $(P>0.200)$ in the mean fluorescence intensity of phagocytic PMNL were detected between non-supplemented cows (basal) and those supplemented with trace minerals from inorganic or organic sources. On day 6 postpartum, the mean fluorescence intensity of phagocytic PMNL from cows supplemented with trace minerals from inorganic sources was $39 \%$ higher $(P=0.002)$ than that of cows receiving no supplemental trace minerals. However, the mean fluorescence intensity of phagocytic PMNL was not different $(P=0.127)$ between cows 
receiving no supplemental trace minerals and those supplemented with trace minerals from organic sources on day 6 postpartum. The mean fluorescence intensity of phagocytic PMNL did not differ $(P=0.134)$ between cows supplemented with trace minerals from inorganic and organic sources on day 6 postpartum. Regardless of trace mineral supplementation, the mean fluorescence intensity of phagocytic PMNL was 35 and $17 \%$ higher on day 58 prepartum, compared with days 7 prepartum $(P<0.001)$ and 6 postpartum $(P=0.005)$, respectively. However, the mean fluorescence intensity of phagocytic PMNL was $26 \%$ higher $(P<0.001)$ on day 6 postpartum compared with day 7 prepartum (Figure 5.4E; main effect of period, $P<0.001$ ). Incubation of PMNL with $50 \mu \mathrm{g} / \mathrm{mL}$ of LPS had no effect on the mean fluorescence intensity of phagocytic PMNL (Figure 5.4F, $P=0.415)$. 
Figure 5.4. Effect of trace mineral supplementation source on neutrophil (PMNL) phagocytosis of Escherichia coli particles. Cows were fed basal diets with no added dietary $\mathrm{Mn}, \mathrm{Co}, \mathrm{Cu}$ and $\mathrm{Zn}$ (basal, $\mathrm{n}=13$ ) or supplemented with $200 \mathrm{mg} \mathrm{Mn}, 25 \mathrm{mg} \mathrm{Co}$, $125 \mathrm{mg} \mathrm{Cu}$, and $360 \mathrm{mg} \mathrm{Zn}$ from either inorganic (inorganic, $\mathrm{n}=11$ ) or organic sources (organic, $\mathrm{n}=11$ ). Treatments were administered to the cows between days $57.14 \pm 1.0$ prior to parturition until day 8 after parturition. PMNL were collected from the blood of cows on days $58.14 \pm 1.0$ (pre-treatment sample), $7.30 \pm 0.7$ prior to parturition and 6.37 \pm 0.1 after parturition. Subsequently, the PMNL were incubated with 0 or $50 \mu \mathrm{g} / \mathrm{mL}$ of $E$. coli lipopolysaccharide (LPS) for $120 \mathrm{~min}$. The percentage of PMNL positive for phagocytized E. coli particles (panels A, B, and C) and their geometric mean fluorescence intensity (MFI; panels D, E, and F) were determined by flow cytometry. (A) Effect of interaction of trace mineral supplementation source by period on the percentage of PMNL positive for phagocytized E. coli particles, $P=0.098$. (B) Main effect of period on the percentage of PMNL positive for phagocytized E. coli particles, $P<0.001$. (C) Main effect of LPS on the percentage of PMNL positive for phagocytized E. coli particles, $P=0.580$. (D) Effect of interaction of trace mineral supplementation source by period on the MFI of PMNL positive for phagocytized particles, $P=0.007$. (E) Main effect of period on the MFI of PMNL positive for phagocytized particles, $P<0.001$. (F) Main effect of LPS on the MFI of PMNL positive for phagocytized particles, $P=0415$. Bars in panels $\mathrm{A}, \mathrm{B}$, and $\mathrm{C}$ represent the adjusted least square means and the error bars indicate the standard error of the means. Bars in panels D, E, and F represent adjusted least square means back-transformed from the logarithmic scale and the error bars indicate confidence intervals. *Asterisks indicate difference from basal trace mineral supplementation within the same period at $P<0.05$. ${ }^{\mathrm{a}, \mathrm{b}}$ Bars with different letters differ at $P<0.05$ (Tukey). 

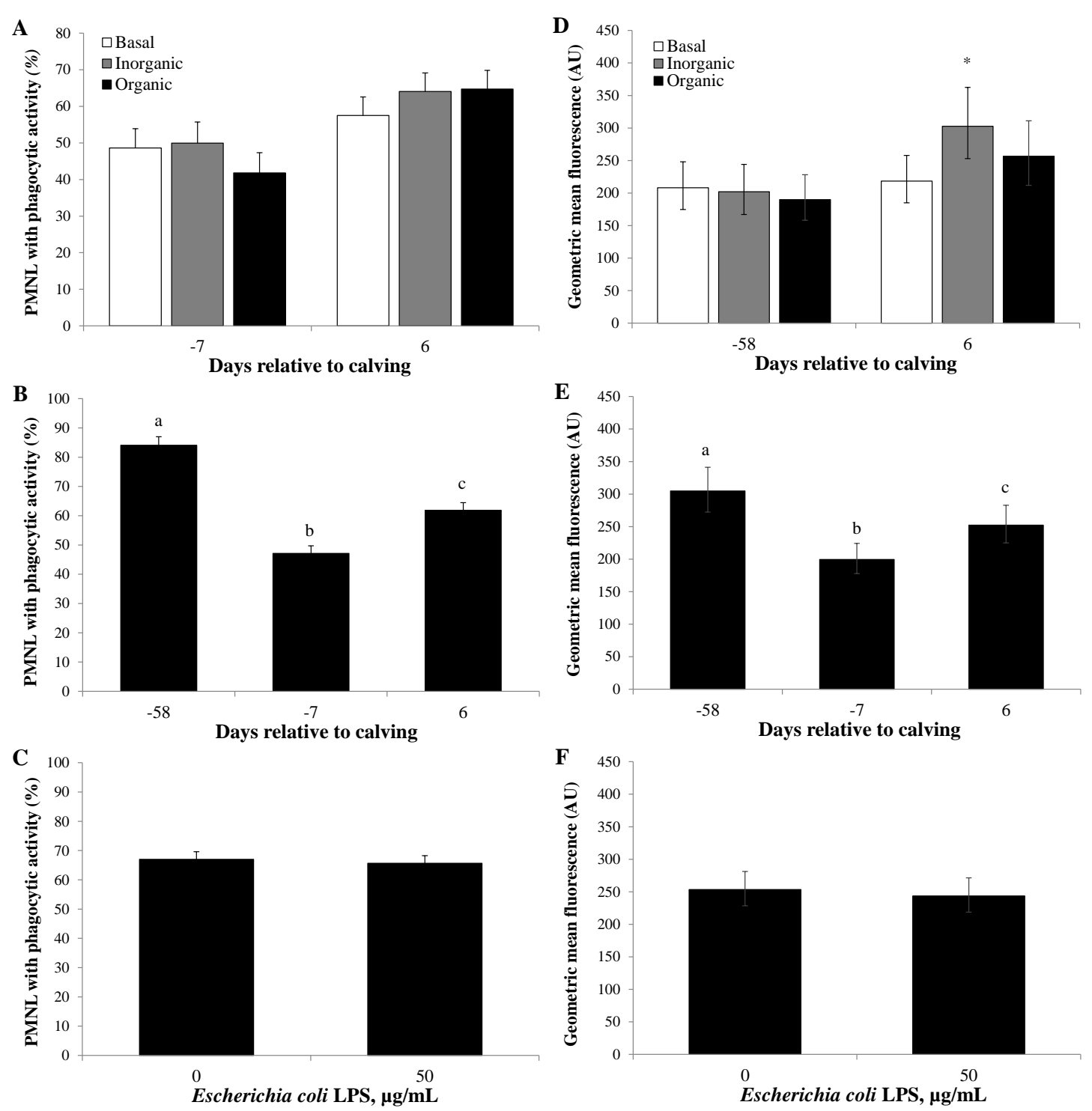


\section{Killing of Escherichia coli}

An effect of interaction of trace mineral supplementation source by period on the PMNL killing ability against $E$. coli was detected (Figure 5.5A, $P<0.001$ ). However, there were no differences $(P>0.100)$ in PMNL killing of $E$. coli between basal, inorganic and organic trace mineral supplemented groups within each period. Similarly, there was no main effect of trace mineral supplementation source on PMNL killing of E. coli (data not shown, $P=0.487)$. Regardless of trace mineral supplementation source, PMNL killing of E. coli was 13 and $20 \%$ lower on day 58 prepartum compared to day 7 prepartum $(P<0.001)$ and day 6 postpartum $(P<0.001)$. Also, killing of $E$. coli by PMNL was $6 \%$ lower $(P=0.021)$ on day 7 prepartum compared with day 6 postpartum (Figure 5.5B; main effect of period, $P<0.001$ ). Incubation of PMNL with $50 \mu \mathrm{g} / \mathrm{mL}$ of LPS had no effect on PMNL killing of $E$. coli (Figure 5.5C, $P=0.727$ ). 
Figure 5.5. Effect of trace mineral supplementation source on the neutrophil (PMNL) killing ability against Escherichia coli. Cows were fed basal diets with no added dietary $\mathrm{Mn}, \mathrm{Co}, \mathrm{Cu}$ and $\mathrm{Zn}$ (basal, $\mathrm{n}=13$ ) or supplemented with $200 \mathrm{mg} \mathrm{Mn}, 25 \mathrm{mg}$ Co, $125 \mathrm{mg} \mathrm{Cu}$, and $360 \mathrm{mg} \mathrm{Zn}$ from either inorganic (inorganic, $\mathrm{n}=11$ ) or organic sources (organic, $\mathrm{n}=11$ ). Treatments were administered to the cows between days $57.14 \pm 1.0$ prior to parturition until day 8 after parturition. PMNL were collected from the blood of cows on days $58.14 \pm 1.0$ (pre-treatment sample), $7.30 \pm 0.7$ prior to parturition and 6.37 \pm 0.1 after parturition. Subsequently, the PMNL were incubated with 0 or $50 \mu \mathrm{g} / \mathrm{mL}$ of E. coli lipopolysaccharide (LPS) for $120 \mathrm{~min}$. The ability of PMNL to kill E. coli was determined by calculating the difference in the number of bacteria incubated in the absence and presence of PMNL and dividing this difference by the number of bacteria incubated in the absence of PMNL. (A) Effect of interaction of trace mineral supplementation source by period on the PMNL killing ability against E. coli, $P<0.001$. (B) Main effect of period on the PMNL killing ability against E. coli, $P<0.001$. (C) Main effect of LPS on the PMNL killing ability against E. coli, $P=0.727$. Bars represent the adjusted least square means and the error bars indicate the standard error of the means. ${ }^{\mathrm{a}, \mathrm{b}}$ Bars with different letters differ at $P<0.05$ (Tukey). 

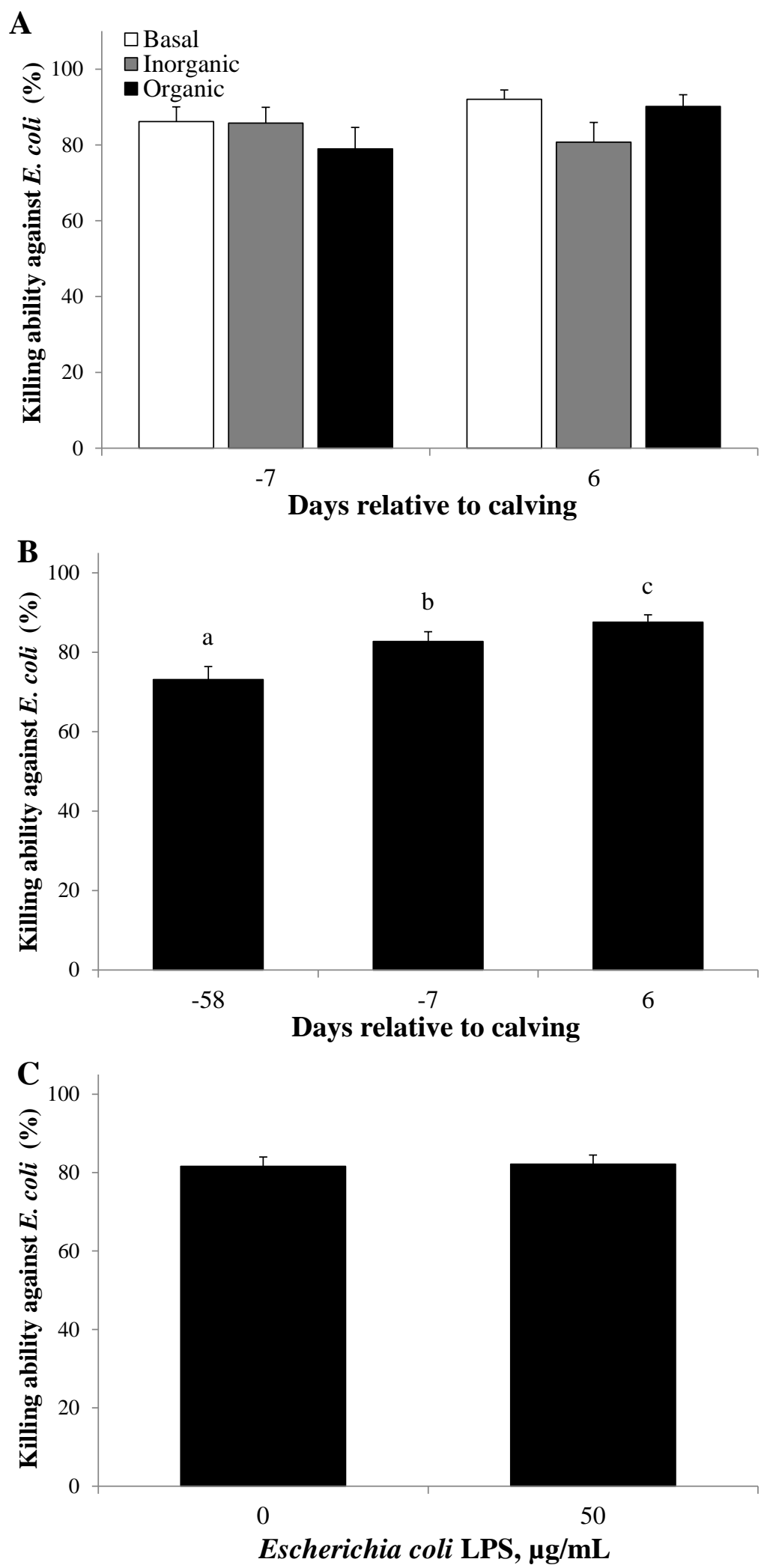


\section{DISCUSSION}

In the current study, minimal differences in PMNL function were detected between cows receiving no added trace minerals or cows supplemented with $\mathrm{Mn}, \mathrm{Co}, \mathrm{Cu}$ and $\mathrm{Zn}$ from either inorganic or organic sources. Cows supplemented with trace minerals from inorganic sources had a 39\% increase in the MFI of phagocytic PMNL on day 6 postpartum, compared with cows receiving no trace mineral supplementation. However, the MFI of phagocytic PMNL from cows supplemented with trace minerals from organic sources was not different compared with that of animals in the basal and inorganic treatment groups. No changes in PMNL ROS production, NETs release, chemotaxis, percent of phagocytic PMNL, and killing of E. coli in response to trace mineral supplementation amount or source were detected. Several studies have reported that replacing supplemental $\mathrm{Mn}, \mathrm{Co}, \mathrm{Cu}$, and $\mathrm{Zn}$ in inorganic forms with equivalent amounts of these trace minerals from organic sources improve the performance of dairy cows (Ballantine et al., 2002; Hackbart et al., 2010; Formigoni et al., 2011; Nocek et al., 2006; Siciliano-Jones et al., 2008). However, there is limited evidence indicating a beneficial effect of trace mineral supplementation from organic sources on innate immune function, at least when compared with trace minerals from inorganic sources supplemented at the same level. Kincaid et al. (1997) reported no differences in phagocytosis and killing of $S$. aureus by PMNL collected from calves receiving no added dietary $\mathrm{Zn}, 150 \mathrm{ppm}$ of $\mathrm{Zn}$ as an amino acid complex, $300 \mathrm{ppm}$ of $\mathrm{Zn}$ as an amino acid complex or $300 \mathrm{ppm}$ of $\mathrm{Zn}$ as $\mathrm{Zn}$ oxide. Most experiments have investigated the effects of organic trace mineral supplementation on milk SCC. For instance, Kellogg et al. (2004), in a summary of 12 trails, reported a 33\% reduction in SCC in the milk of animals supplemented with $\mathrm{Zn}$ 
methionine, compared to those supplemented inorganic forms of $\mathrm{Zn}$. However, it is unclear if this response was the result of higher content of $\mathrm{Zn}$ in the diets fed to the $\mathrm{Zn}$ methionine group in 7 of those trials. Other researchers (Ballantine et al., 2002; Formigoni et al., 2011; Hackbart et al., 2010; Siciliano-Jones et al., 2008) have found no differences in milk SCC between animals supplemented with equivalent amounts of trace minerals from inorganic or organic sources. Recently, Formigoni et al. (2011) reported that replacing inorganic forms of $\mathrm{Zn}, \mathrm{Cu}$ and $\mathrm{Mn}$ with similar amounts of these trace minerals from organic sources increased the content of total Ig in colostrum by $19 \%$, suggesting that organic trace minerals may enhance the transfer of passive immunity to the calf. Furthermore, numerous reports indicate that dietary deficiencies of individual trace minerals, especially $\mathrm{Cu}$, impair bovine $\mathrm{PMNL}$ function and the animal's resistance to infection. For instance, dairy heifers fed a basal diet containing $6.5 \mathrm{ppm}$ of $\mathrm{Cu}$, had a more severe clinical response to experimental E. coli mastitis, compared with animals supplemented with $20 \mathrm{ppm}$ of $\mathrm{Cu}$ (Scaletti et al., 2003). In addition, induced $\mathrm{Cu}$ deficiency in cattle results in defective killing of $S$. aureus (Xin et al., 1991) and $C$. albicans (Boyne and Arthur, 1981; Sharma et al., 2005), and reduced SOD and cytochrome $c$ activities (Cerone et al., 2000; Xin et al., 1991). In addition, steers receiving a Co-deficient diet for 72 weeks had reduced PMNL killing capacity against $C$. albicans, whereas Co-repleted animals recovered their antimicrobial capacity 4 weeks after supplementation with Co from inorganic sources (Paterson and MacPherson, 1990). These studies suggest a consistent impairment of PMNL function when the animals were induced to develop a severe deficiency, frequently accomplished via addition of other elements that interfere with the absorption and bioavailability of trace minerals. Instead 
of a severe deficiency, the cows in our study that received no added dietary trace minerals likely developed a marginal deficiency which did not impair most aspects of PMNL function, but only resulted in a minimal reduction in phagocytosis on day 6 postpartum. Indeed, homeostatic regulation of trace mineral metabolism such as changes in absorption, storage in tissues and excretion ensure no disturbances in biological function during suboptimal intake (Miller, 1975). During periods of limited supply of trace minerals, dairy cows may prioritize the routing of trace minerals towards immune function over processes less critical for survival (Suttle and Jones, 1989). Alternatively, it is possible that the periparturient alteration in PMNL function is caused by other firstlimiting variables not associated with these trace minerals. Furthermore, our findings suggest that increasing the bioavailability of dietary trace minerals by replacing inorganic with organic forms does not support augmented PMNL function in healthy animals. However, as discussed by Suttle and Jones (1989), lack of effect on immune function tested in vitro may not reflect the potential of improved trace mineral metabolism to enhance the animal's resistance to disease. Therefore, there is need for studies to determine the role of trace mineral supplementation on the susceptibility of dairy cows to challenge infections.

The total production of ROS by PMNL was assessed by a luminol-dependent assay that measures the intracellular and extracellular generation of $\mathrm{O}_{2}^{-}, \mathrm{OH}^{-}, \mathrm{H}_{2} \mathrm{O}_{2}$, $\mathrm{ONOO}^{-}$, and $\mathrm{HClO}$ (Rinaldi et al., 2007). Plotted luminescence values measured every 5 min for 240 min were used to calculate the AUC that represents the overall amount of ROS produced, the maximal level of ROS generated and the time at which PMNL reached maximal ROS generation. No differences in any of these parameters of PMNL 
ROS generation were detected between cows receiving no added trace minerals and those supplemented with $\mathrm{Mn}, \mathrm{Co}, \mathrm{Cu}$ and $\mathrm{Zn}$ from either inorganic or organic sources. To our knowledge, the effects of trace mineral supplementation source on bovine PMNL ROS production have not been examined. However, severe dietary deficiencies of individual trace minerals have been demonstrated to impair the activity of enzymes involved in the production of PMNL ROS. For instance, PMNL collected from dairy steers fed a $\mathrm{Cu}$ depleting diet for 8 months had a $48 \%$ reduction in SOD activity, compared with PMNL from steers supplemented with $20 \mathrm{ppm}$ of $\mathrm{Cu}$ as $\mathrm{Cu}$ sulfate (Xin et al., 1991). Similarly, PMNL from $\mathrm{Cu}$-depleted Angus calves had a 32\% decrease in their cytochrome $c$ oxidase activity, relative to $\mathrm{PMNL}$ from animals receiving $\mathrm{Cu}$ only from forages and concentrates (Cerone et al., 2000). In humans, Moroni et al. (2005) reported that PMNL from old healthy donors with reduced intracellular $\mathrm{Zn}$ content had a $25 \%$ reduction in their production of ROS. Although this finding does not imply a cause-effect relationship, the authors reported a significant negative correlation between zinc content in PMNL and oxidative burst $(r=-0.6)$. As discussed earlier, it is likely that the cows receiving no added trace minerals in our study only developed a marginal dietary deficiency which did not alter the capacity of PMNL to produce ROS. Furthermore, independent of trace mineral supplementation, the generation of ROS by PMNL decreased as the cow approaches parturition and reached a lowest level on the first week after calving, in agreement with previous research (Dosogne et al., 1999; Hoeben et al., 2000; Mehrzad et al., 2001b, 2002; Revelo and Waldron, 2010). Reduced generation of ROS by PMNL was characterized by a decreased total amount over time (AUC), reduced maximal ROS production, and delayed response of PMNL to stimulus (i.e., increased time to maximal 
ROS). Incubation of PMNL with $50 \mu \mathrm{g} / \mathrm{mL}$ of LPS did not affect the amount and maximal production of ROS, but decreased the time needed for the PMNL to reach maximal ROS. Recently, we reported that LPS enhances the generation of ROS and gene expression of the cytokines IL-8, tumor necrosis factor, and superoxide dismutase, an enzyme involved in the generation of ROS (Revelo and Waldron, 2012).

A novel antimicrobial mechanism termed neutrophil extracellular traps (NETs) has been recently described (Brinkmann et al., 2004). Activation of PMNL leads to swelling of the nuclei and dissolution of its chromatin to form strands of decondensed DNA. Although it was initially proposed that NETs are released by intact viable cells, recent evidence indicates that PMNL extrude NETs in a processes of cell death termed NETosis which is different from apoptosis and necrosis (Remijsen et al., 2011). Rupture of nuclear and granular membranes while the integrity of the plasma membrane is maintained, allows mixing of these structures with granule substances including proteolytic enzymes, antimicrobial proteins, and ROS. Subsequently, these NETs are released to the extracellular matrix where they contribute to the killing ability of PMNL by delivering a high concentration of antimicrobial substances and act as a barrier that prevents the microorganisms from spreading (Brinkmann and Zychlinsky, 2007). In contrast to other PMNL antimicrobial mechanisms, NET formation is not affected by incubation in milk, suggesting that NET release by bovine PMNL may be a strategic tactic to defeat the invading pathogen during an IMI (Lippolis et al., 2006b). In the present study, no differences in the release of NETs by PMA- and non-stimulated PMNL were detected between cows receiving no added trace minerals or supplemented with trace minerals from inorganic or organic sources. Regardless of trace mineral 
supplementation, PMA- and non-stimulated PMNL collected on day 6 postpartum had higher release of NETs compared with PMNL collected on days 7 and 58 prepartum. We previously reported that PMNL collected from cows on day 7 postpartum was $27 \%$ lower compared with day 12 prepartum (Revelo and Waldron, 2010). The reason for the discrepancy between these studies using similar assays to measure NETs is unclear, but it is possible that the expression of NETs varies substantially during the days around parturition. Incubation of PMNL with $50 \mu \mathrm{g} / \mathrm{mL}$ of LPS for $120 \mathrm{~min}$ had no effect of NETs release by PMA-stimulated PMNL. However, LPS increased NET release by nonstimulated PMNL in agreement with a recent study by our group (Revelo and Waldron, 2012).

No difference in PMNL chemotaxis towards IL-8 was detected between cows receiving no added trace minerals and those supplemented with trace minerals from either inorganic or organic sources. Arthington et al. (1996) reported that PMNL from AngusHereford heifers with a severe $\mathrm{Cu}$-deficiency had similar chemotactic capacity towards complement C5a compared with PMNL from animals fed a diet supplemented with 10 ppm of $\mathrm{Cu}$, suggesting that PMNL chemotaxis may not be impaired by severe $\mathrm{Cu}$ deficiencies. In our study, PMNL chemotaxis decreased by $18 \%$ between day 58 prepartum and 6 postpartum but was not different between day 7 prepartum and 6 postpartum. Previous research has reported lower chemotaxis towards complement C5a by PMNL collected on day 3 postpartum, compared with PMNL collected from cows on day 150 postpartum (Stevens et al., 2011). In contrast, no change in PMNL directed migration has been observed between 5 weeks prior to parturition and 5 weeks after parturition (Detilleux et al., 1995; Kehrli and Goff, 1989). Together, these results indicate 
that the ability of PMNL to migrate towards a chemoattractant decreases as the dairy cow transitions between midlactation into the prepartum period, but does not change around parturition. In agreement with a previous report (Revelo and Waldron, 2012), a 120-min incubation of PMNL with $50 \mu \mathrm{g} / \mathrm{mL}$ of LPS had no effect on chemotaxis towards IL-8.

The percentage of PMNL positive for intracellular E. coli bioparticles did not differ between cows fed no added trace minerals and animals supplemented with Mn, Co, $\mathrm{Cu}$ and $\mathrm{Zn}$ from either inorganic or organic sources. However, the MFI of phagocytic PMNL was $12 \%$ higher in cows supplemented with trace minerals from inorganic sources on day 6 postpartum, compared to animals in the basal treatment group. In contrast, Kincaid et al. (1997) reported no differences in the phagocytic ability against S. aureus between PMNL collected from calves receiving no added dietary $\mathrm{Zn}$ and animals supplemented with equivalent amounts of $\mathrm{Zn}$ from either organic or organic sources. Also, previous reports indicate that cattle with induced Cu-deficiency do not have impaired PMNL phagocytic abilities (Boyne and Arthur, 1981; Xin et al., 1991). In our study, no differences in the MFI of phagocytic PMNL were detected between cows in the organic and inorganic treatment groups, or between organic and basal groups. Because trace minerals from organic sources have increased bioavailability compared with minerals from inorganic sources (Predieri et al., 2005; Wedekind et al., 1992), we hypothesized that PMNL from cows supplemented with trace minerals from organic sources would have improved phagocytic abilities, compared with PMNL from basal and inorganic treatment groups. The reason why PMNL from cows supplemented trace minerals from inorganic sources had a slight improvement in phagocytic capacity, whereas PMNL from cows supplemented trace minerals from organic sources had no 
improvement, remains unclear. Regardless of trace mineral supplementation source, the percentage of PMNL positive for E. coli bioparticles and their MFI decreased between days 58 and 7 prepartum and then slightly increased by day 6 postpartum. The phagocytic abilities of PMNL did not recover to the levels found on day 58 prepartum. Studies investigating the changes in bovine PMNL phagocytic abilities during the periparturient period have yielded conflicting results. Several investigators have reported an increase in PMNL phagocytosis between week 1 prepartum and week 1 postpartum (Guidry et al., 1976; Detilleux et al., 1995; Dosogne et al., 1999), consistent with our results. In contrast, no change in phagocytic activity during the periparturient period has been reported as well (Cai et al., 1994; Daniel et al., 1991; Dosogne et al., 2001). The majority of these experiments have measured changes in PMNL phagocytosis during the 5 weeks around the time of calving. Conversely, our findings also indicate that the phagocytic ability of PMNL decreases between approximately 8 weeks prepartum and the periparturient period.

The killing capacity of PMNL against E. coli did not differ between cows fed no added trace minerals and animals supplemented with $\mathrm{Mn}, \mathrm{Co}, \mathrm{Cu}$ and $\mathrm{Zn}$ from inorganic or organic sources. Similarly, Kincaid et al. (1997) reported no difference in PMNL killing ability against $S$. aureus between PMNL collected from calves receiving no added dietary $\mathrm{Zn}$ and animals supplemented with equivalent amounts of $\mathrm{Zn}$ either as an amino acid complex or $\mathrm{Zn}$ oxide. Several studies indicate that severe deficiencies of $\mathrm{Cu}$ (Boyne and Arthur, 1981; Sharma et al., 2005; Xin et al., 1991) and Co (MacPherson et al., 1987; Paterson and MacPherson, 1990) impair the killing ability of PMNL. As previously discussed, the difference in results reported in the current study and those obtained in 
experiments with induced trace mineral depletions, may be attributable to the magnitude and duration of the dietary deficiency. Furthermore, the killing ability of PMNL against E. coli slightly increased between days 58 and 7 prepartum, and augmented again by day 6 postpartum. Other investigators have reported no changes (Kehrli et al., 1989; Cai et al., 1994) or a decrease in the killing capacity of PMNL between several weeks before parturition and the early postpartum periods (Daniel et al., 1991; Detilleux et al., 1995; Dosogne et al., 2001). In agreement with the current study, recent research from our group (Revelo and Waldron, 2010) indicated that the killing capacity of PMNL against $S$. aureus increases between days 12 prepartum and 7 postpartum. As discussed in the report from our earlier study (Revelo and Waldron, 2010), differences in methodologies used to determine the antimicrobial capacity of PMNL could explain the inconsistency of results regarding the antimicrobial capacity of PMNL. Finally, as recently reported by our laboratory (Revelo and Waldron, 2012), incubation of PMNL with $50 \mu \mathrm{g} / \mathrm{mL}$ of LPS for 120 min had no effect on the killing capacity of PMNL against E.coli.

\section{CONCLUSIONS}

Dietary supplementation of dairy cows with $200 \mathrm{mg} \mathrm{Mn}, 25 \mathrm{mg} \mathrm{Co}, 125 \mathrm{mg} \mathrm{Cu}$, and $360 \mathrm{mg} \mathrm{Zn}$ from either inorganic or organic sources had minimal effects on the function of PMNL during the periparturient period. Only a slight increase in the amount of $E$. coli particles ingested by PMNL was detected in the animals were supplemented trace minerals from inorganic forms, compared with cows receiving no dietary added trace minerals. There was no difference in any parameter of PMNL function between animals supplemented with trace minerals from inorganic and those supplemented with equivalent 
amounts of trace minerals from organic forms. These results indicate that replacing inorganic forms of trace mineral with more bioavailable forms such as those from organic sources results in no changes in basal PMNL function. The occurrence of only a small alteration in PMNL phagocytosis in the cows receiving no added dietary trace minerals compared to those supplemented with trace minerals from inorganic sources, suggests that homeorhetic mechanisms may be able to prevent most disturbances in PMNL function during suboptimal intake of these micronutrients or that other variables not associated with these trace minerals are first-limiting in the function of PMNL around the time of calving. Regardless of trace mineral supplementation, the production of ROS, chemotaxis towards IL-8, and phagocytosis of E. coli particles by PMNL were compromised in the periparturient period and may contribute to the increased incidence and severity of IMI observed during this time. 


\title{
CHAPTER SIX
}

\section{EFFECT OF TRACE MINERAL SUPPLEMENTATION SOURCE ON THE GLOBAL GENE EXPRESSION PROFILE OF NEUTROPHILS COLLECTED FROM PERIPARTURIENT DAIRY COWS}

\begin{abstract}
The objective of this study was to investigate the effects of trace mineral supplementation from inorganic or organic sources on the global gene expression in neutrophils (PMNL) collected from dairy cows during the periparturient period. Twenty one pregnant Holstein cows entering second or greater lactation were randomly assigned to a basal diet with no added dietary $\mathrm{Mn}, \mathrm{Co}, \mathrm{Cu}$ and $\mathrm{Zn}$ (basal, $\mathrm{n}=7$ ) or supplemented with $200 \mathrm{mg} \mathrm{Mn}, 25 \mathrm{mg} \mathrm{Co}, 125 \mathrm{mg} \mathrm{Cu}$, and $360 \mathrm{mg} \mathrm{Zn}$ from sulfate and carbonate sources (inorganic, $\mathrm{n}=7$ ) or supplied by amino acid complexes of $\mathrm{Mn}, \mathrm{Cu}$, and $\mathrm{Zn}$, and cobalt glucoheptonate (organic, $\mathrm{n}=7$ ). Total supplementation for inorganic and organic cows was brought to $65,15,75$, and $2.5 \mathrm{ppm}$ of diet using the inorganic sources added to each gelcap for $\mathrm{Mn}, \mathrm{Cu}, \mathrm{Zn}$, and $\mathrm{Co}$, respectively. Oral treatments were administered between day $57.1 \pm 1$ prior to parturition until day 8 after parturition. PMNL were harvested from the blood of cows on days $58.1 \pm 1.0$ prepartum (pre-treatment sample) and $6.4 \pm 0.1$ after parturition. Subsequently, PMNL were incubated with 0 or $50 \mu \mathrm{g} / \mathrm{mL}$ of Escherichia coli lipopolysaccharide (LPS) for $120 \mathrm{~min}$. Messenger RNA (poly-A enriched) was purified from the PMNL and converted into a library of template molecules for high throughput RNA-sequencing. Sequenced fragments were then mapped to the bovine reference genome. The resulting digital count data was analyzed to
\end{abstract}


determine the effects of trace mineral supplementation source and physiological state on the global gene expression profile of non-activated and LPS-activated PMNL. Relative to cows fed the basal diet, trace mineral supplementation from either inorganic or organic sources resulted in minimal changes in gene expression in non-activated and LPSactivated PMNL. However, when compared with trace minerals from inorganic sources, organic trace mineral supplementation altered the expression of 230 and 47 genes in nonactivated and LPS-activated PMNL, respectively. Pathway analysis revealed that organic trace mineral supplementation (relative to inorganic) up-regulated genes that enriched the RIG-I-like receptor signaling, cytosolic DNA-sensing, and TOLL-like receptor (TLR) signaling pathways. The RIG-I-like receptor signaling and cytosolic DNA-sensing pathways were up-regulated in both, non-activated and LPS-activated PMNL whereas the TLR signaling pathway was only enriched in non-activated PMNL. Although trace mineral supplementation, regardless of source, had minimal effects on PMNL gene expression, these results suggest that organic trace minerals (vs. inorganic) stimulate gene pathways involved in antigen recognition and immune response. Relative to PMNL from prepartum cows, PMNL collected from postpartum animals had altered expression of 9,589 and 2,001 transcripts in non-activated and LPS-activated PMNL, respectively. Down-regulated pathways important for immune function included the chemokine signaling, leukocyte transendothelial migration, Fc gamma R-mediated phagocytosis, cell adhesion molecules (CAMs), TLR signaling, lysosome, apoptosis, and NOD-like receptor signaling pathways. Overall, we discovered an extensive collection of genes and pathways with altered expression in PMNL from postpartum (vs. prepartum) cows that 
highlight the molecular mechanisms of PMNL dysfunction during the periparturient period. 


\section{INTRODUCTION}

Trace minerals can influence immune cell function of dairy cows directly or due to their antioxidant properties. Numerous trace minerals including $\mathrm{Co}, \mathrm{Zn}$, and $\mathrm{Mn}$ can either function as components of enzymes involved in the antioxidant defense system or act as scavengers of free radicals (Spears and Weiss, 2008). In addition to these traditional roles as anti-oxidants, trace minerals such as $\mathrm{Zn}$ can directly influence immune cells via regulation of gene expression, hematopoiesis and cytokine production (Haase and Rink, 2009; Prasad, 2007). In dairy cows, supplementation of trace minerals has traditionally relied on inorganic salts including sulfates, oxides, and carbonates (Andrieu, 2008). However, feeding trace minerals bound to an organic compound may result in increased bioavailability in part due to their resistance to antagonisms in the rumen (Wedekind et al., 1992; Spears, 2003; Predieri et al., 2005). Replacing $\mathrm{Mn}, \mathrm{Cu}$, and $\mathrm{Zn}$, and Co from inorganic sources with equivalent amounts of these trace minerals as organic complexes has increased milk yield (Ballantine et al., 2002; Hackbart et al., 2010; Nocek et al., 2006), and fat content (Formigoni et al., 2011; Siciliano-Jones et al., 2008). In addition to enhanced performance, trace mineral supplementation from organic sources may improve some aspects of immune function in dairy cows. For example, substituting trace minerals from inorganic sources with trace minerals as organic complexes increased the content of immunoglobulins (Ig) in colostrum (Formigoni et al., 2011). Recently, our group investigated the effects of trace mineral supplementation source on the function of polymorphonuclear leukocytes or neutrophils (PMNL). In this study, replacing inorganic forms of trace mineral with the same amount of trace minerals from organic sources had no effect on the PMNL production of reactive oxygen species 
(ROS), neutrophil extracellular traps (NETs), chemotaxis, phagocytosis, or killing of $E$. coli. (Revelo et al., chapter dissertation chapter five). However, it is possible that trace mineral supplementation from organic sources may improve neutrophil performance via other mechanisms that may be revealed by changes in global gene expression.

Neutrophils are essential cells of the bovine innate immune system, forming the first line of defense against pathogens during intramammary infections. The competence by which these PMNL recognize and destroy the invading pathogen often determines the severity and duration of the infection (Sordillo and Streicher, 2002). This protection against pathogens, however, is not without cost because the uncontrolled influx of PMNL into the mammary gland may lead to severe host tissue damage caused by toxic levels of oxygen free radicals (Zhao and Lacasse, 2008). Remarkably, the function of PMNL is altered during the periparturient period of dairy cows (Kehrli et al., 1989; Cai et al., 1994; Revelo and Waldron, 2010) and may contribute to the increased incidence and severity of mastitis during this period (Mallard et al., 1998). In addition, dairy cows experience higher oxidative stress during this periparturient period as evidenced by increased circulatory thiobarbituric acid-reactive substances and reactive oxygen metabolites (Bernabucci et al., 2005). When these oxidant molecules are not effectively removed by the antioxidant system, oxidative stress may further compromise the health of the dairy cow (Miller et al., 1993). Therefore, the protective role of antioxidant mechanisms including those supported by trace minerals is essential during the periparturient period when the dairy cow experiences augmented oxidative stress (Sordillo et al., 2007).

Neutrophils are generally described as terminally differentiated effector cells with a short half-life and limited transcriptional capabilities. However, these cells express a 
wide range of genes encoding proteins involved in phagocytosis, respiratory burst activity, and cytokine synthesis (Newburger et al., 2000). In dairy cows, extensive research has focused on the antimicrobial function of PMNL during the periparturient period. However, only a few experiments have investigated the global gene expression profile in PMNL using high-throughput technologies which allow the screening of large numbers of genes simultaneously. For instance, Madsen et al. (2004) used microarrays to investigate the genetic expression profile of blood neutrophils around the time of parturition and reported that calving induces a shift in the expression of a major cluster of genes towards prolonged survival of PMNL. Although hybridization-based methods such as the microarray used by Madsen et al. (2004) enabled whole genome studies in a variety of organisms, novel high-throughput sequencing technologies such as RNAsequencing (RNA-seq) have overcome the limitations presented by hybridization-based methods and offer increased performance to unveil differential gene expression (Wang et al., 2009a). Therefore, the objective of this study was to determine the effects of trace mineral supplementation from either inorganic or organic sources on the global gene expression profile of neutrophils collected in the prepartum (pre-treatment) and postpartum periods. Furthermore, we aimed to test the effects of trace mineral supplementation source in both resting and lipopolysaccharide (LPS)-stimulated PMNL. It was hypothesized that (1) PMNL from dairy cows supplemented with amino acid complexes of $\mathrm{Mn}, \mathrm{Cu}$, and $\mathrm{Zn}$, and $\mathrm{Co}$ glucoheptonate would have an altered gene expression profile towards an improved immune response; and (2) Non-activated and LPS-activated PMNL collected from postpartum cows would have an altered gene 
expression profile consistent with the defective PMNL function observed around parturition.

\section{MATERIALS AND METHODS}

\section{Experimental Design, Animals, and Dietary Treatments}

The use and care of all animals were approved by The University of Missouri Animal Care and Use Committee. All cows enrolled in the study did not experience any major clinical event during the previous lactation, had an average previous lactation somatic cell count lower than 300,000 cells/ml and a current locomotion score lower than two (Sprecher et al., 1997). All cows were fed a basal diet formulated to meet or exceed nutritional recommendations for all nutrients except $\mathrm{Mn}, \mathrm{Co}, \mathrm{Cu}$, and $\mathrm{Zn}$ (Table 6.1; NRC, 2001). Cows were assigned to one of three treatments: no added dietary Mn, Co, $\mathrm{Cu}$ and $\mathrm{Zn}$ (basal, $\mathrm{n}=7$ ), additional $\mathrm{Mn}, \mathrm{Co}, \mathrm{Cu}$ and $\mathrm{Zn}$ from inorganic sources (inorganic, $\mathrm{n}=7$ ) and additional $\mathrm{Mn}, \mathrm{Co}, \mathrm{Cu}$ and $\mathrm{Zn}$ from organic sources (organic, $\mathrm{n}=$ 7). Treatments were administered orally once per day via gelcap capsules using a balling gun. Animals in the control (basal) group received a cornmeal carrier devoid of $\mathrm{Mn}$, Co, $\mathrm{Cu}$ and $\mathrm{Zn}$ and only obtained these trace minerals from dietary forages and concentrates in the basal diet. Cows assigned to trace mineral supplementation from inorganic sources were fed the basal diet supplemented with $200 \mathrm{mg} \mathrm{Mn}, 25 \mathrm{mg} \mathrm{Co}, 125 \mathrm{mg} \mathrm{Cu}$, and 360 mg Zn per day in the form of Mn sulfate, Co carbonate, $\mathrm{Cu}$ sulfate, and $\mathrm{Zn}$ sulfate. Cows assigned to trace mineral supplementation from organic sources were fed the basal diet supplemented with the same amounts of trace minerals in the form of Mn-amino acid complex (Availa-Mn ${ }^{\circledR}$, Zinpro Corp., Eden Prairie, MN), Co glucoheptonate $\left(\mathrm{COPRO}^{\circledR}\right.$, 
Zinpro Corp.), Cu-amino acid complex (Availa-Cu ${ }^{\circledR}$, Zinpro Corp.), and Zn-amino acid complex (Availa-Mn ${ }^{\circledR}$, Zinpro Corp.). Cows on both the inorganic and organic treatments were further supplemented with these four trace minerals from inorganic sources added to each gelcap such that total daily supplementation was $65,2.5,15$, and $75 \mathrm{ppm}$ of the total daily DMI. This level of trace mineral supplementation to the animals in the inorganic and organic treatment groups met or exceeded NRC (2001) nutritional recommendations for dairy cattle. 
Table 6.1. Chemical composition of the basal prepartum and postpartum diets (DM basis)

\begin{tabular}{|c|c|c|c|}
\hline Nutrient & Far-off prepartum ${ }^{1,2,3}$ & Close-up prepartum & Postpartum \\
\hline Crude Protein, \% & 12.8 & 13.7 & 15.7 \\
\hline Available Protein, \% & 11.5 & 12.3 & 14.5 \\
\hline ADICP, \% & 1.3 & 1.4 & 1.1 \\
\hline Acid Detergent Fiber, \% & 37.0 & 35.1 & 22.1 \\
\hline Neutral Detergent Fiber, \% & 55.3 & 51.2 & 31.6 \\
\hline Crude Fat, \% & 2.1 & 2.0 & 4.3 \\
\hline TDN, \% & 56.8 & 58.3 & 72.3 \\
\hline NEL, Mcal/kg & 1.3 & 1.3 & 1.7 \\
\hline $\mathrm{NEm}, \mathrm{Mcal} / \mathrm{kg}$ & 1.1 & 1.2 & 1.7 \\
\hline $\mathrm{NEg}, \mathrm{Mcal} / \mathrm{kg}$ & 0.6 & 0.6 & 1.1 \\
\hline Soluble Protein, \% CP & 22.2 & 23.8 & 37.5 \\
\hline Lignin, \% & 6.6 & 6.0 & 3.7 \\
\hline NDICP, \% & 5.2 & 4.9 & 3.4 \\
\hline Starch, \% & 5.8 & 10.1 & 29.2 \\
\hline $\mathrm{NFC}, \%$ & 26.0 & 29.0 & 44.5 \\
\hline Calcium, \% & 0.64 & 0.83 & 0.91 \\
\hline Phosphorus, \% & 0.21 & 0.23 & 0.27 \\
\hline Magnesium, \% & 0.36 & 0.40 & 0.35 \\
\hline Potassium, \% & 1.50 & 1.38 & 1.29 \\
\hline Sodium, \% & 0.11 & 0.12 & 0.30 \\
\hline Iron, $\mathrm{ppm}$ & 249.5 & 220.3 & 233.8 \\
\hline Zinc, ppm & 31.0 & 32.7 & 36.0 \\
\hline Copper, ppm & 11.0 & 10.8 & 9.8 \\
\hline Manganese, ppm & 81.8 & 72.0 & 35.8 \\
\hline Molybdenum, ppm & 1.4 & 1.3 & 1.6 \\
\hline Cobalt, ppm & 0.1 & 0.2 & 0.2 \\
\hline Sulfur, \% & 0.3 & 0.3 & 0.2 \\
\hline Ash, $\%$ & 8.9 & 9.0 & 7.3 \\
\hline
\end{tabular}

${ }^{\mathrm{T}}$ Values represent averages of samples composited every $4 \mathrm{wk}$.

${ }^{2}$ Basal diets were formulated to meet or exceed nutritional recommendations for all nutrients except $\mathrm{Mn}, \mathrm{Co}, \mathrm{Cu}$, and $\mathrm{Zn}$ (NRC, 2001).

${ }^{3}$ Cows received no dietary added (basal, $\mathrm{n}=7$ ) or additional $200 \mathrm{mg} \mathrm{Mn}, 25 \mathrm{mg}$ Co, 125 $\mathrm{mg} \mathrm{Cu}$, and $360 \mathrm{mg} \mathrm{Zn}$ per day in the form of $\mathrm{Mn}$ sulfate, Co carbonate, $\mathrm{Cu}$ sulfate, and $\mathrm{Zn}$ sulfate (inorganic, $\mathrm{n}=7$ ) or $\mathrm{Mn}$-amino acid complex, Co glucoheptonate, $\mathrm{Cu}$-amino acid complex, and Zn-amino acid complex (organic; Zinpro Corp.; $\mathrm{n}=7$ ). The level of trace mineral supplementation from inorganic and organic sources met or exceeded NRC (2001) nutritional recommendations. 
Cows were housed in a free-stall barn, milked twice daily and fed individually for ad libitum feed intake via Calan doors (American Calan, Northwood, NH). Animals were enrolled on the study 65 days before expected parturition and removed from the experiment on day 8 after calving. Dietary treatments were initiated on day $57.1 \pm 1$ prepartum and ended on day 8 postpartum. To determine the effect of trace mineral supplementation from inorganic and organic sources on the genetic profile of circulating PMNL, approximately $450 \mathrm{~mL}$ of blood were collected on days $58.1 \pm 1.0$ prepartum (pre-treatment sample) and $6.4 \pm 0.1$ postpartum. Blood was collected via jugular vein catheters into sterile $500 \mathrm{~mL}$ polypropylene conical tubes (Corning Inc., NY) containing $75 \mathrm{~mL}$ of acid-citrate dextrose, inverted 3 times. Catheters were inserted using aseptic techniques and immediately removed after collection of samples. Blood samples stored on ice until isolation of blood PMNL (<20 min).

\section{Isolation of Bovine Neutrophils}

Neutrophils were isolated using procedures modified from Weber et al. (2001) as validated in our laboratory. The $500 \mathrm{~mL}$ conical tubes (Corning Inc.) containing the blood sample were centrifuged at $1,000 \times g$ for $20 \mathrm{~min}$ at $4^{\circ} \mathrm{C}$ to separate plasma from the cell pack. Plasma was discarded and the remaining cells were suspended in $1450 \mathrm{~mL}$ of calcium and magnesium-free Hanks' Balanced Salt Solution (CMF-HBSS; Sigma Chemical Co.). The suspension was gently mixed and $40 \mathrm{~mL}$ aliquots were pipetted down the side of a $50-\mathrm{mL}$ polypropylene conical tube containing $10 \mathrm{~mL}$ of $1.084 \mathrm{~g} / \mathrm{mL}$ of Percoll $^{\circledR}$ (GE Healthcare Bio-Sciences Corp., Piscataway, NJ). The cells were then subjected to gradient centrifugation $\left(400 \times \mathrm{g}\right.$ for $40 \mathrm{~min}$ at $\left.22^{\circ} \mathrm{C}\right)$. The supernatant, mononuclear cell layer and Percoll ${ }^{\circledR}$ were aseptically aspirated and discarded. The pellet 
(PMNL and erythrocytes) was retained and erythrocytes were lysed by hypotonic shock. For this procedure, two volumes of an ice-cold $10.56 \mathrm{~m} M \mathrm{Na}_{2} \mathrm{HPO}_{4}, 2.67 \mathrm{~m} M \mathrm{NaH}_{2} \mathrm{PO}_{4}$ solution were added to one volume of cell suspension and the solution was mixed by gentle aspiration and inversion. Tonicity was restored by the addition of one volume of a $10.56 \mathrm{~m} M \mathrm{Na}_{2} \mathrm{HPO}_{4}, 2.67 \mathrm{~m} M \mathrm{NaH}_{2} \mathrm{PO}_{4} 0.43 \mathrm{M} \mathrm{NaCl}$ solution. The cell solution was then centrifuged $\left(850 \mathrm{x} g\right.$ ) for $4 \mathrm{~min}$ at $4^{\circ} \mathrm{C}$ and the pellet washed twice by resuspension in $35 \mathrm{~mL}$ of CMF-HBSS and recentrifugation $\left(850 \times \mathrm{g}\right.$ for 4 min at $\left.4^{\circ} \mathrm{C}\right)$. Cells were enumerated and viability was determined by propidium iodide (Sigma Chemical Co.) exclusion using a Cellometer Vision ${ }^{\circledR}$ automatic cell counter (Nexcelom Bioscience LLC., Lawrence, MA). The concentration of cells was adjusted and the suspension was kept at $4{ }^{\circ} \mathrm{C}$ until incubation with LPS.

\section{Incubation of PMNL with E. coli Lipopolysaccharide}

The effects of the dietary supplement on the genetic profile of neutrophils were determined in both resting and LPS-stimulated PMNL. Lipopolysaccharide from E. coli (serotype O55:B5, strain CDC 1644-70) was obtained from Sigma Chemical Co. A working solution of $250 \mu \mathrm{g} / \mathrm{mL}$ LPS was added to $50-\mathrm{mL}$ polypropylene conical tubes (Corning Inc., NY) containing purified neutrophils to reach final concentrations of 0 or $50 \mu \mathrm{g} / \mathrm{mL}$ of LPS at a cell concentration of $1.2 \times 10^{7}$ cells $/ \mathrm{mL}$. The tubes were then placed in a humidified incubator $\left(37^{\circ} \mathrm{C}, 5 \% \mathrm{CO}_{2}\right)$ and incubated for 120 minutes while inverted in a Labquake ${ }^{\circledR}$ tube rotator (Barnstead-Thermolyne Corp., Dubuque, IA). Under similar conditions, our laboratory has tested the effects of increasing concentrations of LPS $(0,1,25$ and $50 \mu \mathrm{g} / \mathrm{mL}$ of LPS) on PMNL function and reported that the strongest stimuli was obtained at the $50 \mu \mathrm{g} / \mathrm{mL}$ dose (Revelo and Waldron, 2012). After 
incubation, the cell solutions were centrifuged $(850 \mathrm{x} g)$ for $4 \mathrm{~min}$ at $4^{\circ} \mathrm{C}$. The cell pellet was resuspended in $\operatorname{Trizol}^{\circledR}$ at a concentration of $1 \times 10^{7}$ cells $/ \mathrm{mL}$. This suspension was homogenized using a sonic dismembrator (Sonifier $^{\circledR}$, Branson Ultrasonics Corp., Danbury, CT) and stored at $-80^{\circ} \mathrm{C}$ until mRNA extraction.

\section{mRNA Extraction}

Total cellular RNA was harvested from neutrophils using $1 \mathrm{~mL}$ of Trizol ${ }^{\circledR}$ reagent (Invitrogen Co., Carlsbad, CA) per $1 \times 10^{7}$ cells, according to the manufacturer recommendations. Messenger RNA was further purified using the RNeasy ${ }^{\circledR}$ Mini Kit (Qiagen, Valencia, CA). Genomic DNA was then removed by on-column DNase digestion using the RNase-Free DNase Set (Qiagen) during the RNeasy ${ }^{\circledR}$ procedure. The concentration and purity of RNA were determined with a ND-1000 NanoDrop ${ }^{\circledR}$ spectrophotometer (NanoDrop Technologies, Wilmington, DE). In addition, the integrity

of RNA was assessed by electrophoresis and fluorescence detection using the Experion ${ }^{\mathrm{TM}}$ automated electrophoresis system (Bio-Rad, Hercules, CA). After purification and quality control, RNA samples were stored at $-80^{\circ} \mathrm{C}$ in RNase-free water.

\section{Library Preparation and mRNA Sequencing}

Messenger RNA was converted into a library of template molecules for high throughput cDNA sequencing (Illumina, Inc., Hayward, CA) by the University of Missouri DNA Core following the manufacturer's recommendations. First, poly-A containing mRNA molecules were purified via poly-T oligo-attached magnetic beads using the $\operatorname{TruSeq}^{\circledR}$ RNA sample preparation kit (Illumina, Inc.). Poly-A mRNA molecules were then fragmented into small pieces using divalent cations under high temperatures. Subsequently, first strand cDNA synthesis was performed using reverse 
transcriptase and random primers followed by second strand cDNA synthesis using DNA polymerase I and RNase H. Next, blunt-end fragments are created and the 3 ' ends of these cDNA fragments were adenylated. After indexed adapters were ligated, these products were purified and enriched by PCR to create the final cDNA libraries. The amplified libraries were indexed for multiplexing during this step. The size, purity, and concentration of the final constructs were evaluated using the Agilent BioAnalyzer $2100^{\circledR}$ automated electrophoresis system (Agilent Technologies, Inc., Santa Clara, CA) and the Qubit $^{\circledR}$ fluorometer (Invitrogen, Co.) using the Quant-iT ${ }^{\mathrm{TM}}$ HS dsDNA reagent kit (Invitrogen, Co.). The indexed cDNA libraries were pooled into a single lane (6 libraries per lane) of a flow cell. Single-read sequencing by synthesis was conducted on the Illumina HiSeq 2000 according to the manufacturer's recommendations. Called sequenced reads were approximately 50 bases in length. The resulting data were collected and de-multiplexed using the CASAVA package version 1.8 (Illumina, Inc.) for downstream analysis.

\section{Sequence alignment}

Reads were processed and mapped against a bovine reference genome using the NextGene ${ }^{\circledR}$ sequence analysis software v. 2.15 (SoftGenetics, LLC., State College, PA). First, sequencing output files (FASTQ) were converted to FASTA format. Subsequently, low quality reads were trimmed or removed from the sample file using the Format Conversion tool. Quality thresholds were used to identify low quality reads. Reads were removed from the sample file if they failed to meet one of the following criteria: median quality score $>20,<3$ uncalled bases, $>35$ called bases, and $<3$ consecutive bases with a quality score less than 15 (Table 6.2). Reads that passed the quality filter were then 
aligned to the Bos_taurus_UMD_3.1 reference assembly (available at ftp://ftp.ncbi.nih.gov/genomes/Bos_taurus/) using the Sequence Alignment tool. A seeded alignment was carried out using the first 24 bases with four bases between seed start positions. Reads that mapped equally well to more than 50 genomic regions were discarded. For reads that did not match exactly to the reference, a maximum of two mismatched bases were allowed to align the entire read to the genome. Reads with a matching base percentage lower than $85 \%$ were further filtered out. The average counts for matched and unmatched reads are indicated in Table 6.3. After alignment, expression reports were generated using the gene sequences in the reference assembly as the alignment segments. Digital sequencing read counts were obtained from these reports and used in the statistical analysis. 
Table 6.2. Conversion and quality control summary

\begin{tabular}{lc}
\hline Description & Number $^{1}$ \\
\hline Total sequenced fragments & $24,717,638$ \\
Reads that passed the quality thresholds & $21,621,872$ \\
Reads that failed to pass the quality filter & \\
Median quality score $<20$ & 619,457 \\
Uncalled bases per read > 3 & 24,616 \\
Called bases per read < 35 & 488,170 \\
Three consecutive bases with quality score < 15 & $1,963,523$ \\
Total & $3,095,765$ \\
Reads trimmed due to low base quality score & $4,875,162$ \\
Bases trimmed due to low base quality score & $97,464,113$ \\
Mean read length, bases & 49.0 \\
\hline
\end{tabular}

${ }^{\mathrm{T}}$ Values represent the average of 84 sample files. 
Table 6.3. Summary of sequencing read alignment to the reference bovine genome

\begin{tabular}{lc}
\hline Description $^{1}$ & Number $^{2}$ \\
\hline Total matched reads & $18,937,151$ \\
Perfectly matched reads & $14,909,473$ \\
Total unmatched reads & $2,684,560$ \\
Matched bases & $1,705,378,173$ \\
Unmatched bases recorded as mutations & $3,589,298$ \\
Average read length & 48.6 \\
Average coverage & 6.3 \\
Reference assembly length, bases & $2,670,102,999$ \\
Covered bases & $241,574,935$ \\
Percent coverage & $9.0 \%$ \\
Percentage of reads mapped & \\
1. & $88.0 \%$ \\
\hline
\end{tabular}

${ }^{\mathrm{T}}$ Reads were aligned to the Bos_taurus_UMD_3.1 bovine reference assembly (available at ftp://ftp.ncbi.nih.gov/genomes/Bos_taurus/).

${ }^{2}$ Values represent the average of 84 sample files.

${ }^{4}$ Total reads matched divided by the sum of matched and unmatched reads, multiplied by 100. 


\section{Statistical Analyses}

A modified generalized linear modeling (GLM) approach was used to model the read counts in order to determine which genes were differentially expressed. Traditional GLMs are, as their name implies, extensions of the usual linear model framework (e.g., ANOVA and linear regression) to data that is not normally distributed (non-Gaussian). Several computational modifications and theoretical extensions were made to the traditional GLM framework and its estimation algorithm to make them applicable to high-throughput read count data, as detailed in (McCarthy et al., 2012). These methods are available in the edgeR package (Robinson et al., 2010) available through the opensource Bioconductor/R project. The approach implemented in edgeR (limma-voom pipeline) fitted a separate count model for each gene, but it is done in an empirical Bayes manner which permits a flexible borrowing of information across genes in order to improve the precision of the model estimates. The voom method models the counts after a $\log$ transformation ( $\log y_{g i}+0.5$, where $g$ is a gene in a sample $i$ ) which converge the variables to normality.

For each analysis, models were fit in edgeR using gene-wise estimates of the dispersion. The design matrix incorporated the main effect of trace mineral supplementation (control, inorganic or organic), LPS incubation (0 or $50 \mu \mathrm{g} / \mathrm{mL}$ of LPS), and physiological state (prepartum or postpartum). Fixed cow-level effects were also incorporated to account for the dependency of measurements from the same animal. Model fit was assessed through checks for numerical convergence failure, quantilequantile plots, and goodness-of fit test. To account for pre-existing variation between animals, several measurements of PMNL function obtained from the same cows (Revelo 
et al., dissertation chapter five) were tested as potential covariates. Covariates were selected based on their relationship with gene expression data corresponding to the pretreatment sample. Based on this principle, the baseline measurement of reactive oxygen species (ROS) was used as a covariate in the model. To determine which genes were differentially expressed, an overall F-test of pre-planned contrasts was conducted. To account for multiple testing the $P$-values from the overall F-test were adjusted via the false discovery rate (FDR) approach of Benjamini and Hochberg, which controls the expected FDR. Genes beneath the expected FDR cutoff of $20 \%$ were considered statistically significant. If a gene was significant at a FDR of $20 \%$, then each contrast within that gene was tested. The contrasts in the analysis included: i) contrasts between diets (inorganic vs. control, organic vs. control, and organic vs. inorganic) within each

activation state (50 or $0 \mu \mathrm{g} / \mathrm{mL}$ of LPS); ii) contrasts of the LPS effect (50 vs. $0 \mu \mathrm{g} / \mathrm{mL}$ of LPS) between dietary treatments (inorganic vs. control, organic vs. control, and organic vs. inorganic); and iii) contrasts of physiological states (prepartum vs. postpartum) within each activation state ( 50 or $0 \mu \mathrm{g} / \mathrm{mL}$ of LPS). For this last contrast, only gene expression data from the inorganic group was included in the postpartum dataset to avoid a bias of diet.

\section{Functional Annotation Analyses}

Differentially expressed genes within each contrast were loaded to the DAVID Bioinformatics Resources 6.7 (http://david.abcc.ncifcrf.gov/home.jsp; DAVID Bioinformatics, 2012) for pathway and gene ontology (GO) enrichment analyses as described by Huang et al. (2009a,b). The functional annotation chart module of DAVID was used to identify enriched annotation terms. Differentially regulated genes within each 
contrast were mapped to associated biological annotation GO terms. To indentify overrepresented (enriched) pathways, all genes differentially regulated by a major comparison between dietary treatments were used. Enriched pathways were then visualized using the Kyoto Encyclopedia of Genes and Genomes (http://www.genome.jp/keg; KEGG, 2012) and genes classified according to LPS activation. The enrichment of gene members for each of the annotation terms (e.g. GO terms and pathways) was tested by a modified Fisher's exact test (EASE score) using the Bos taurus genome as a reference or background. For the GO enrichment analysis, GO terms were limited to biological processes and molecular functions. The thresholds to select significant KEGG pathways and GO categories were EASE score of $<0.100$ and count $>4$.

\section{RESULTS}

\section{Differential gene expression}

On average, 24.7 million fragments were sequenced from each cDNA library. Approximately, 21.6 million of these sequenced fragments passed the quality filters (Table 6.2) and 18.9 million of these aligned to the bovine reference genome (Table 6.3). The percent of reads that mapped to the reference was $88 \%$ with a percent base coverage of 9\% (Table 6.3). To adjust for pre-existing differences between dietary treatment groups, analysis of covariance was conducted using measurements of PMNL function and gene expression obtained on day 58 prepartum (pre-treatment). Based on their relationship with pre-treatment gene expression data $(P<0.050)$, ROS was used as a covariate in the analysis of variance $(R=0.632,7962$ genes). After covariate adjustment, 
analysis of variance was conducted on the digital counts. For those genes that showed differential expression in any of the contrasts (overall F-test; $P<0.05,20 \%$ FDR), its statistical significance within each contrast was tested.

Supplemental Table 6.5 lists the genes differentially regulated due to trace mineral supplementation within each comparison. Relative to cows fed the basal diet, trace mineral supplementation from inorganic sources resulted in $6(\uparrow 5, \downarrow 1)$ and $6(\uparrow 6)$ transcripts differentially regulated in non-activated and LPS-activated PMNL, respectively $(P<0.050,20 \%$ FDR; Supplemental Table 6.5A). Alternatively, trace mineral supplementation from organic sources resulted in seven genes differentially regulated in non-activated $(\uparrow 2, \downarrow 5)$, and $4(\uparrow 4)$ in LPS-activated PMNL, relative to the basal treatment group $(P<0.050,20 \%$ FDR; Supplemental Table 6.5B). Relative to cows fed inorganic trace minerals, trace mineral supplementation from organic sources altered the expression of $230(\uparrow 89, \downarrow 141)$ and $47(\uparrow 29, \downarrow 18)$ genes in non-activated and LPSactivated PMNL, respectively $(P<0.050,20 \%$ FDR; Supplemental Table 6.5C). No genes with altered expression were detected $(P<0.050,20 \%$ FDR $)$ within the contrast of LPS effect $(50 / 0 \mu \mathrm{g} / \mathrm{mL}$ of LPS) between dietary treatments (inorganic vs. control, organic vs. control, and organic vs. inorganic).

The top 100 genes differentially regulated due to physiological state in nonactivated and LPS-activated PMNL are indicated in Supplemental Table 6.6. Relative to PMNL from prepartum cows, PMNL collected from postpartum animals had altered expression of 9,589 $(\uparrow 0, \downarrow 9,589)$ and $2,001(\uparrow 801, \downarrow 1,200)$ genes in non-activated and LPS-activated PMNL, respectively ( $<<0.050,1 \%$ FDR; Supplemental Table 6.6). The effect of LPS (50 vs. $0 \mu \mathrm{g} / \mathrm{mL}$ ) was also compared between PMNL from postpartum and 
prepartum cows. In PMNL from postpartum (vs. prepartum cows), incubation with 50 $\mu \mathrm{g} / \mathrm{mL}$ of LPS (vs. $0 \mu \mathrm{g} / \mathrm{mL}$ ) resulted in the up-regulation and down-regulation of 1 and 9,428 genes, respectively ( $\mathrm{P}<0.050,1 \%$ FDR; Supplemental Table 6.6).

\section{Pathway and gene ontology enrichment analysis}

Pathway analysis was performed to identify enriched biological pathways in PMNL due to trace mineral supplementation in PMNL incubated with 0 or $50 \mu \mathrm{g} / \mathrm{mL}$ of LPS. Relative to PMNL from cows fed inorganic trace minerals, three pathways were enriched by up-regulated genes in PMNL due to organic trace mineral supplementation. These included the RIG-I-like receptor signaling, cytosolic DNA-sensing, and TOLL-like receptor (TLR) signaling pathways. In both, non-activated and LPS-activated PMNL, organic trace mineral supplementation stimulated the RIG-I-like receptor signaling (Figure 6.1, $P<0.001$ ) and cytosolic DNA-sensing (Figure 6.2, $P=0.027$ ) pathways. In contrast, the TLR signaling pathway was only enriched in non-activated PMNL (Figure $6.3, P=0.087)$. No pathways were enriched by genes with altered expression when the comparisons were made between the inorganic or organic and the basal dietary treatment $(P>0.100)$. 
Figure 6.1. Regulation of the RIG-I-like receptor signaling pathway in neutrophils (PMNL) from cows supplemented with trace minerals from organic sources relative to those fed inorganic trace minerals. Cows were fed a basal diet with no added dietary Mn, $\mathrm{Co}, \mathrm{Cu}$ and $\mathrm{Zn}$ (basal, $\mathrm{n}=7$ ) or supplemented with $200 \mathrm{mg} \mathrm{Mn}, 25 \mathrm{mg} \mathrm{Co}, 125 \mathrm{mg} \mathrm{Cu}$, and $360 \mathrm{mg} \mathrm{Zn}$ from sulfate and carbonate sources (inorganic, $\mathrm{n}=7$ ) between day $57.1 \pm$ 1 prior to parturition until day 8 after parturition. PMNL were harvested from the blood of cows on day $6.4 \pm 0.1$ after parturition and incubated with 0 or $50 \mu \mathrm{g} / \mathrm{mL}$ of Escherichia coli lipopolysaccharide (LPS) for $120 \mathrm{~min}$ to determine their global gene expression using RNA-sequencing. Genes with altered expression in response to organic relative to inorganic trace mineral supplementation were loaded to DAVID Bioinformatics Resources 6.7 where KEGG pathway analysis was applied to uncover enriched functional pathways. Objects highlighted in orange represent up-regulated gene products in both, LPS- and non-activated PMNL except for TRIM25 (only in nonactivated PMNL). Green boxes correspond to gene products specific for the Bos Taurus genome that were unaltered by treatments. Significance of gene-pathway enrichment conducted with a modified Fisher's exact test (EASE score, $P<0.001$ ). 


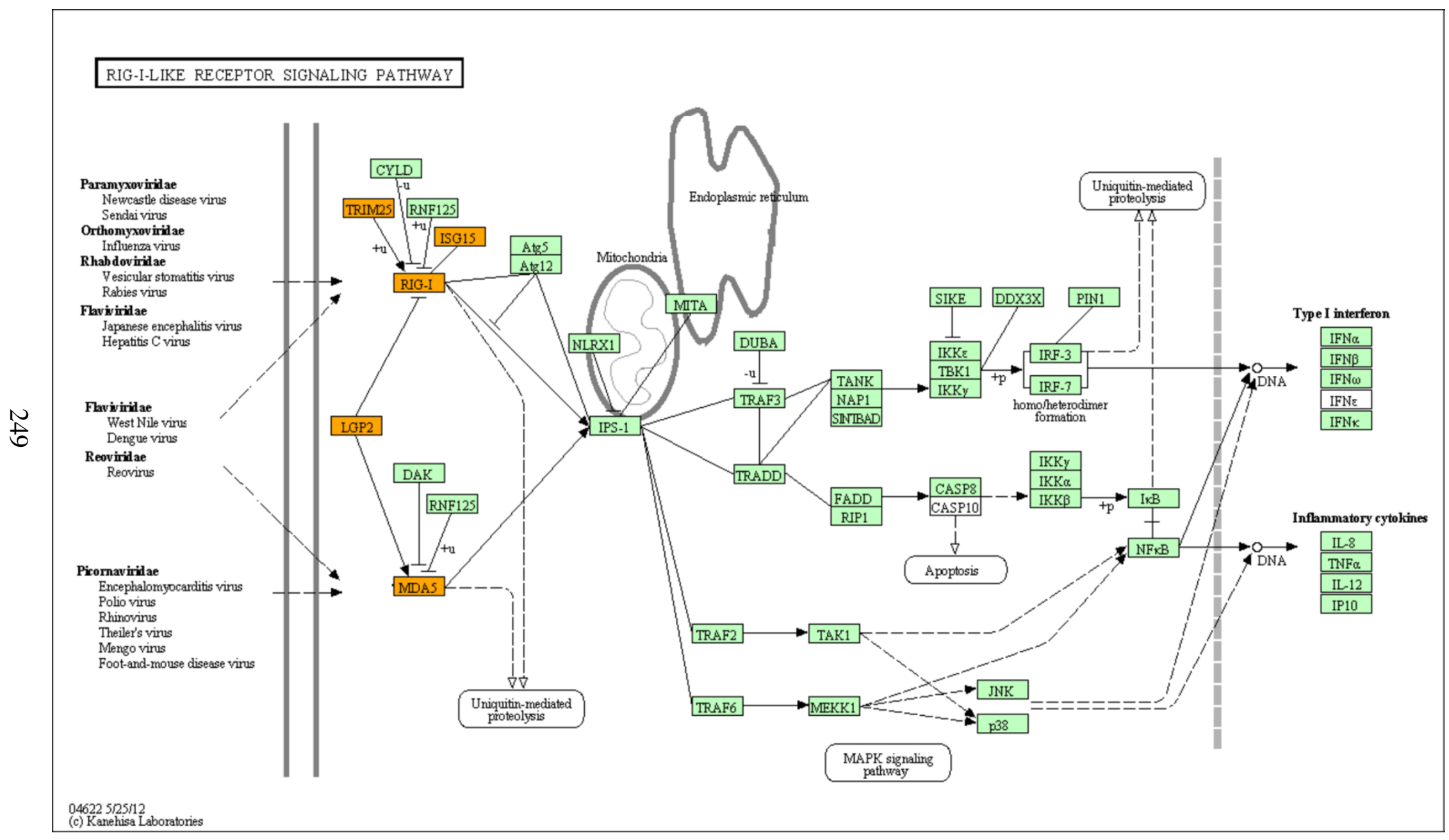


Figure 6.2. Regulation of the cytosolic DNA-sensing pathway in neutrophils (PMNL) from cows supplemented with trace minerals from organic sources relative to those fed inorganic trace minerals. Cows were fed a basal diet with no added dietary $\mathrm{Mn}$, $\mathrm{Co}, \mathrm{Cu}$ and $\mathrm{Zn}$ (basal, $\mathrm{n}=7$ ) or supplemented with $200 \mathrm{mg} \mathrm{Mn}, 25 \mathrm{mg} \mathrm{Co}, 125 \mathrm{mg} \mathrm{Cu}$, and $360 \mathrm{mg} \mathrm{Zn}$ from sulfate and carbonate sources (inorganic, $\mathrm{n}=7$ ) between day $57.1 \pm$ 1 prior to parturition until day 8 after parturition. PMNL were harvested from the blood of cows on day $6.4 \pm 0.1$ after parturition and incubated with 0 or $50 \mu \mathrm{g} / \mathrm{mL}$ of Escherichia coli lipopolysaccharide (LPS) for $120 \mathrm{~min}$ to determine their global gene expression using RNA-sequencing. Genes with altered expression in response to organic relative to inorganic trace mineral supplementation were loaded to DAVID Bioinformatics Resources 6.7 where KEGG pathway analysis was applied to uncover enriched functional pathways. Objects highlighted in orange represent up-regulated gene products in both, LPS- and non-activated PMNL. Green boxes correspond to gene products specific for the Bos Taurus genome that were unaltered by treatments. Significance of gene-pathway enrichment conducted with a modified Fisher's exact test (EASE score, $P=0.027$ ). 


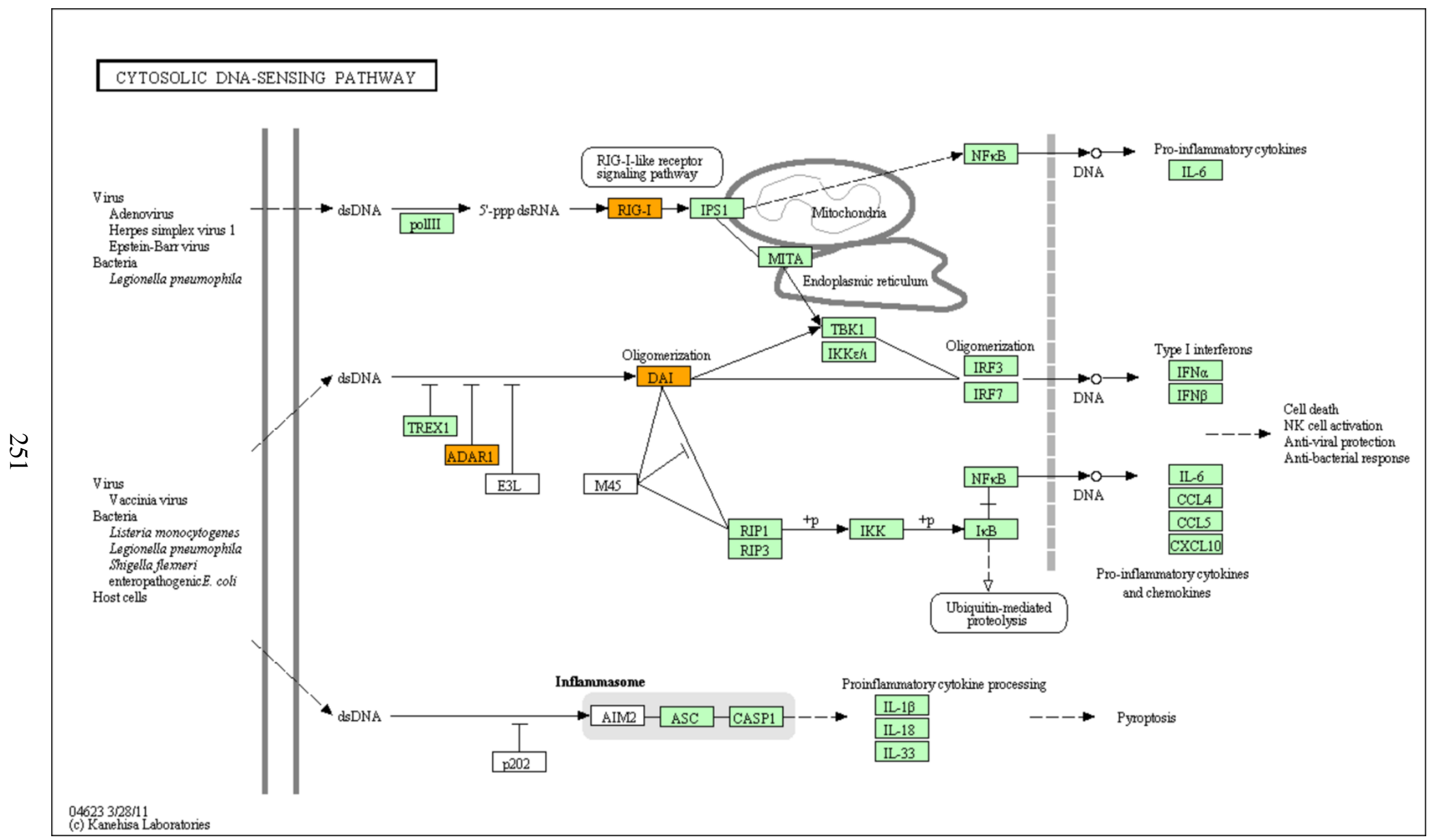


Figure 6.3. Regulation of the TOLL-like receptor signaling pathway in neutrophils (PMNL) from cows supplemented with trace minerals from organic sources relative to those fed inorganic trace minerals. Cows were fed a basal diet with no added dietary Mn, Co, Cu and Zn (basal, n = 7) or supplemented with $200 \mathrm{mg} \mathrm{Mn,} 25 \mathrm{mg}$ Co, $125 \mathrm{mg} \mathrm{Cu}$, and $360 \mathrm{mg} \mathrm{Zn}$ from sulfate and carbonate sources (inorganic, $\mathrm{n} \mathrm{=7}$ ) between day $57.1 \pm 1$ prior to parturition until day 8 after parturition. PMNL were harvested from the blood of cows on day $6.4 \pm 0.1$ after parturition and incubated with 0 or $50 \mu \mathrm{g} / \mathrm{mL}$ of Escherichia coli lipopolysaccharide (LPS) for $120 \mathrm{~min}$ to determine their global gene expression using RNA-sequencing. Genes with altered expression in response to organic relative to inorganic trace mineral supplementation were loaded to DAVID Bioinformatics Resources 6.7 where KEGG pathway analysis was applied to uncover enriched functional pathways. Objects highlighted in orange represent upregulated gene products only in non-activated PMNL. Green boxes correspond to gene products specific for the Bos Taurus genome that were unaltered by treatments. Significance of gene-pathway enrichment conducted with a modified Fisher's exact test (EASE score, $P=0.087$ ). 


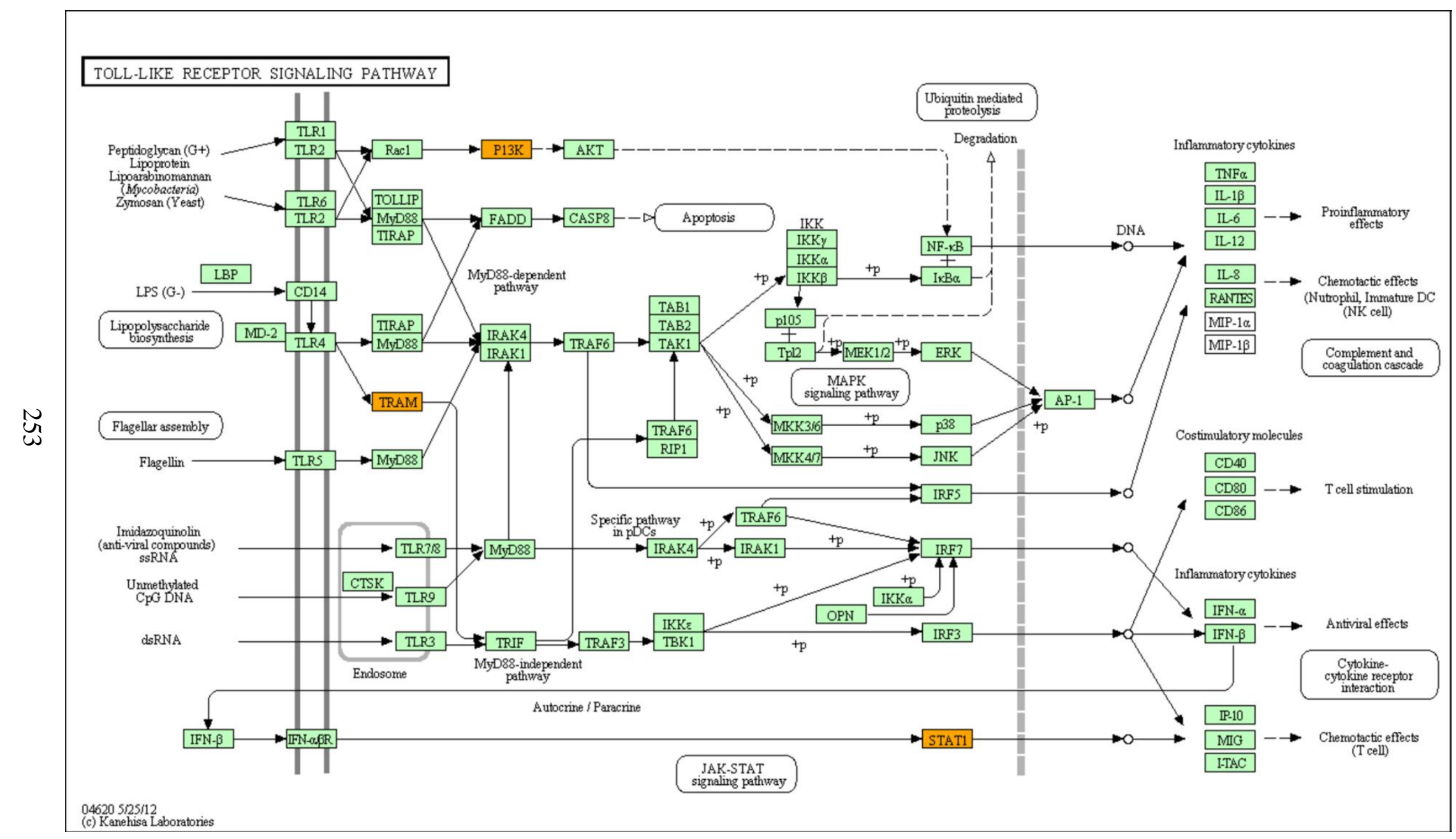


Pathways enriched by genes differentially regulated due to physiological state (postpartum vs. prepartum) are listed in Table 6.4. Non-activated PMNL from postpartum cows had a total of 39 pathways enriched by down-regulated genes, compared with nonactivated PMNL from prepartum cows $(P<0.100$; Table 6.4A). The top three pathways in this group included the MAPK signaling, chemokine signaling pathway, and regulation of actin cytoskeleton pathways. Table 6.4B indicates the 17 pathways enriched by genes differentially expressed due to physiological state in LPS-activated PMNL $(P<0.100)$. In non-activated PMNL, several key pathways relevant to immune cell were down-regulated in the postpartum, compared with the prepartum physiological state. These included the chemokine signaling $(P<0.001$, Figure 6.4), leukocyte transendothelial migration $(P<$ 0.001, Figure 6.5), and Fc gamma R-mediated phagocytosis $(P<0.001$, Figure 6.6) pathways. The top three pathways enriched by up-regulated genes in LPS-activated PMNL from postpartum, relative to prepartum cows, were purine metabolism, glycolysisgluconeogenesis, and fatty acid metabolism. Conversely, the top three pathways enriched by down-regulated genes in LPS-activated PMNL from postpartum, relative to prepartum cows, included spliceosome, RNA degradation, and purine metabolism. Dependent on activation with LPS (50 vs. $0 \mu \mathrm{g} / \mathrm{mL}$ of LPS), 11 pathways were enriched by downregulated genes in PMNL from postpartum, relative to those from prepartum cows $(P<$ 0.100, Table 6.4C). Similarly to LPS-activated PMNL, the top 3 pathways in this group included spliceosome, RNA degradation, and purine metabolism. 
Table 6.4. Enriched pathways for genes with differential expression due to physiological state (postpartum vs. prepartum) after incubation with 0 or $50 \mu \mathrm{g} / \mathrm{mL}$ of $E$. coli lipopolysaccharide (LPS)

\begin{tabular}{|c|c|c|c|}
\hline Pathway & $\uparrow$ or $\downarrow^{1}$ & Genes & $\mathrm{P}_{\text {value }}{ }^{2}$ \\
\hline \multicolumn{4}{|l|}{ A. Non-activated PMNL $(0 \mu \mathrm{g} / \mathrm{ml}$ of LPS $)$} \\
\hline MAPK signaling pathway & $\downarrow$ & 75 & $<0.001$ \\
\hline Chemokine signaling pathway & $\downarrow$ & 62 & $<0.001$ \\
\hline Regulation of actin cytoskeleton & $\downarrow$ & 58 & $<0.001$ \\
\hline Focal adhesion & $\downarrow$ & 49 & $<0.001$ \\
\hline Endocytosis & $\downarrow$ & 45 & 0.003 \\
\hline Leukocyte transendothelial migration & $\downarrow$ & 41 & $<0.001$ \\
\hline Neurotrophin signaling pathway & $\downarrow$ & 40 & $<0.001$ \\
\hline Jak-STAT signaling pathway & $\downarrow$ & 36 & 0.003 \\
\hline Fc gamma R-mediated phagocytosis & $\downarrow$ & 35 & $<0.001$ \\
\hline Cell adhesion molecules (CAMs) & $\downarrow$ & 34 & 0.005 \\
\hline Tight junction & $\downarrow$ & 33 & 0.004 \\
\hline Ubiquitin mediated proteolysis & $\downarrow$ & 33 & 0.016 \\
\hline $\mathrm{T}$ cell receptor signaling pathway & $\downarrow$ & 32 & 0.001 \\
\hline Axon guidance & $\downarrow$ & 32 & 0.002 \\
\hline Toll-like receptor signaling pathway & $\downarrow$ & 30 & $<0.001$ \\
\hline Natural killer cell mediated cytotoxicity & $\downarrow$ & 30 & 0.002 \\
\hline B cell receptor signaling pathway & $\downarrow$ & 29 & $<0.001$ \\
\hline Lysosome & $\downarrow$ & 27 & 0.042 \\
\hline Vascular smooth muscle contraction & $\downarrow$ & 26 & 0.035 \\
\hline Pancreatic cancer & $\downarrow$ & 25 & $<0.001$ \\
\hline Phosphatidylinositol signaling system & $\downarrow$ & 25 & $<0.001$ \\
\hline Acute myeloid leukemia & $\downarrow$ & 24 & $<0.001$ \\
\hline Chronic myeloid leukemia & $\downarrow$ & 24 & 0.001 \\
\hline Prostate cancer & $\downarrow$ & 23 & 0.015 \\
\hline Adipocytokine signaling pathway & $\downarrow$ & 22 & 0.001 \\
\hline Long-term depression & $\downarrow$ & 22 & 0.002 \\
\hline Apoptosis & $\downarrow$ & 22 & 0.022 \\
\hline NOD-like receptor signaling pathway & $\downarrow$ & 21 & $<0.001$ \\
\hline Renal cell carcinoma & $\downarrow$ & 21 & 0.003 \\
\hline Small cell lung cancer & $\downarrow$ & 21 & 0.031 \\
\hline Fc epsilon RI signaling pathway & $\downarrow$ & 20 & 0.024 \\
\hline ErbB signaling pathway & $\downarrow$ & 20 & 0.045 \\
\hline RIG-I-like receptor signaling pathway & $\downarrow$ & 19 & 0.009 \\
\hline Adherens junction & $\downarrow$ & 19 & 0.020 \\
\hline Non-small cell lung cancer & $\downarrow$ & 18 & 0.002 \\
\hline
\end{tabular}


Table 6.4 (continued)

\begin{tabular}{lccc}
\hline Pathway & $\uparrow$ or $\downarrow^{1}$ & Genes & P value \\
\hline Long-term potentiation & $\downarrow$ & 18 & 0.012 \\
Glioma & $\downarrow$ & 18 & 0.014 \\
Inositol phosphate metabolism & $\downarrow$ & 17 & 0.011 \\
mTOR signaling pathway & $\downarrow$ & 16 & 0.014
\end{tabular}

\section{B. LPS-activated PMNL (50 $\mu \mathrm{g} / \mathrm{ml}$ of LPS)}

Purine metabolism

Glycolysis / Gluconeogenesis

Fatty acid metabolism

Hedgehog signaling pathway

Amyotrophic lateral sclerosis (ALS)

Sulfur metabolism

Spliceosome

RNA degradation

Purine metabolism

Pyrimidine metabolism

Cytosolic DNA-sensing pathway

Steroid biosynthesis

$\mathrm{N}$-Glycan biosynthesis

DNA replication

Inositol phosphate metabolism

Homologous recombination

Notch signaling pathway

$\begin{array}{lcr}\uparrow & 10 & 0.070 \\ \uparrow & 7 & 0.011 \\ \uparrow & 5 & 0.040 \\ \uparrow & 5 & 0.087 \\ \uparrow & 5 & 0.097 \\ \uparrow & 3 & 0.044 \\ \downarrow & 30 & <0.001 \\ \downarrow & 14 & <0.001 \\ \downarrow & 14 & 0.077 \\ \downarrow & 10 & 0.070 \\ \downarrow & 9 & 0.006 \\ \downarrow & 8 & <0.001 \\ \downarrow & 8 & 0.007 \\ \downarrow & 7 & 0.011 \\ \downarrow & 7 & 0.082 \\ \downarrow & 6 & 0.016 \\ \downarrow & 6 & 0.086\end{array}$

C. LPS activation effect (50 vs. $0 \mu \mathrm{g} / \mathrm{mL}$ of LPS)

Spliceosome

RNA degradation

Purine metabolism

Pyrimidine metabolism

Cytosolic DNA-sensing pathway

Steroid biosynthesis

N-Glycan biosynthesis

DNA replication

Inositol phosphate metabolism

Homologous recombination

Notch signaling pathway

$\begin{array}{rcr}\downarrow & 30 & <0.001 \\ \downarrow & 14 & <0.001 \\ \downarrow & 14 & 0.077 \\ \downarrow & 10 & 0.070 \\ \downarrow & 9 & 0.006 \\ \downarrow & 8 & <0.001 \\ \downarrow & 8 & 0.007 \\ \downarrow & 7 & 0.011 \\ \downarrow & 7 & 0.082 \\ \downarrow & 6 & 0.016 \\ \downarrow & 6 & 0.086\end{array}$

${ }^{1}$ Direction of gene expression change (postpartum vs. prepartum) indicated with a $\uparrow$ or $\downarrow$.

${ }^{2}$ Significance of enrichment tested with a modified Fisher's exact test (EASE score). 
Figure 6.4. Non-activated neutrophils (PMNL) from postpartum cows have a defective chemokine signaling pathway compared with PMNL from prepartum animals. PMNL were harvested from the blood of cows on days $58.1 \pm 1.0$ prepartum $(\mathrm{n}=21)$, and $6.4 \pm 0.1$ postpartum $(\mathrm{n}=7)$ and incubated with 0 or $50 \mu \mathrm{g} / \mathrm{mL}$ of Escherichia coli lipopolysaccharide (LPS) for $120 \mathrm{~min}$ to determine their global gene expression using RNA-sequencing. Genes with down-regulated expression in non-activated PMNL from postpartum, relative to prepartum cows, were loaded to DAVID Bioinformatics Resources 6.7 where KEGG pathway analysis was applied to uncover enriched functional pathways. Objects highlighted in red represent down-regulated gene products. Green boxes correspond to gene products specific for the Bos Taurus genome that were unaltered by treatments. Significance of gene-pathway enrichment conducted with a modified Fisher's exact test (EASE score, $P<0.001$ ). 


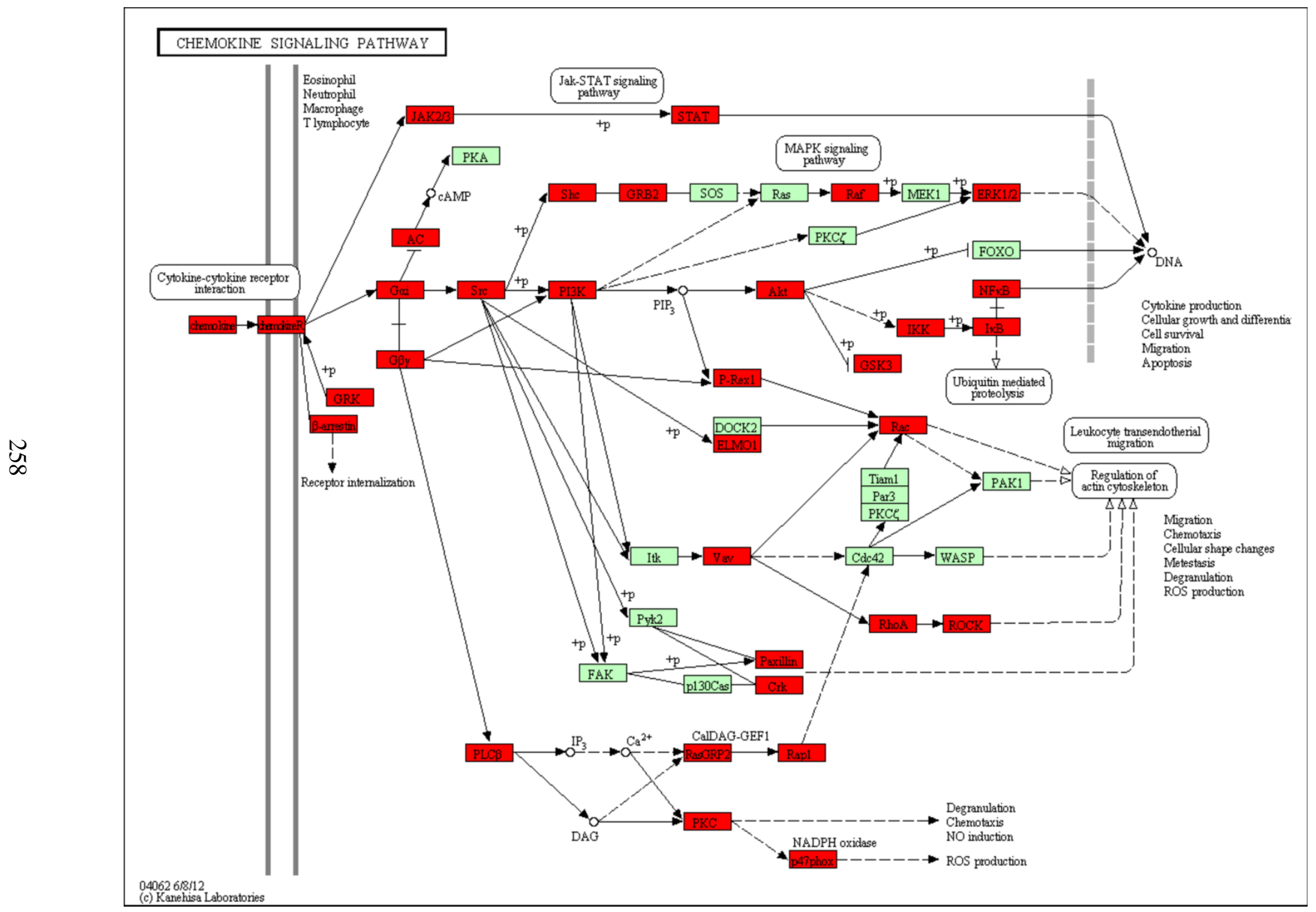


Figure 6.5. Non-activated neutrophils (PMNL) from postpartum cows have a defective leukocyte transendothelial migration pathway compared with PMNL from prepartum animals. PMNL were harvested from the blood of cows on days $58.1 \pm 1.0$ prepartum $(\mathrm{n}=21)$, and $6.4 \pm 0.1$ postpartum $(\mathrm{n}=7)$ and incubated with 0 or $50 \mu \mathrm{g} / \mathrm{mL}$ of Escherichia coli lipopolysaccharide (LPS) for $120 \mathrm{~min}$ to determine their global gene expression using RNA-sequencing. Genes with down-regulated expression in nonactivated PMNL from postpartum, relative to prepartum cows, were loaded to DAVID Bioinformatics Resources 6.7 where KEGG pathway analysis was applied to uncover enriched functional pathways. Objects highlighted in red represent down-regulated gene products. Green boxes correspond to gene products specific for the Bos Taurus genome that were unaltered by treatments. Significance of gene-pathway enrichment conducted with a modified Fisher's exact test (EASE score, $P<0.001$ ). 


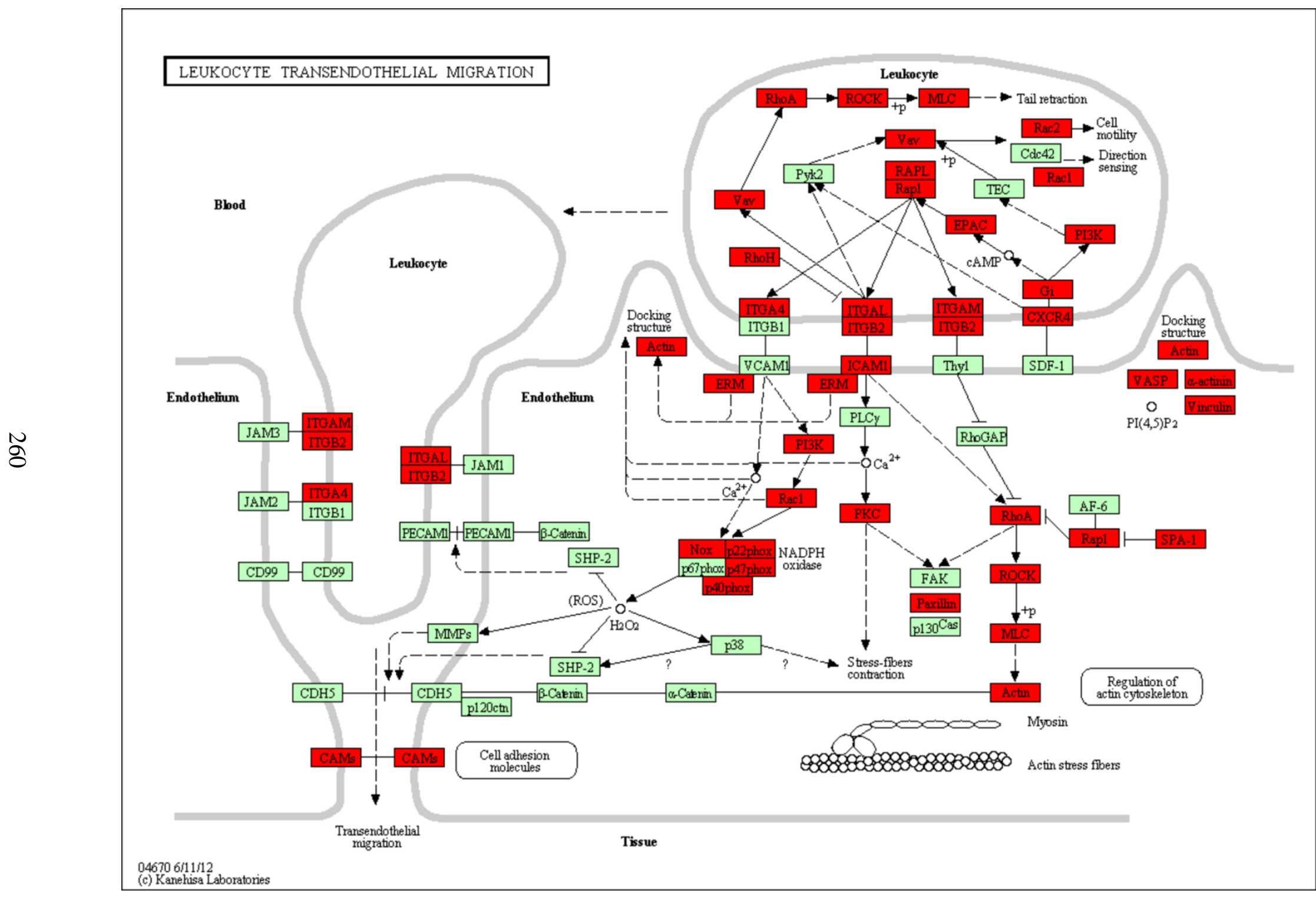


Figure 6.6. Non-activated neutrophils (PMNL) from postpartum cows have a defective Fc gamma R-mediated phagocytosis pathway compared with PMNL from prepartum animals. PMNL were harvested from the blood of cows on days $58.1 \pm 1.0$ prepartum $(\mathrm{n}=21)$, and $6.4 \pm 0.1$ postpartum $(\mathrm{n}=7)$ and incubated with 0 or $50 \mu \mathrm{g} / \mathrm{mL}$ of Escherichia coli lipopolysaccharide (LPS) for $120 \mathrm{~min}$ to determine their global gene expression using RNA-sequencing. Genes with down-regulated expression in nonactivated PMNL from postpartum, relative to prepartum cows, were loaded to DAVID Bioinformatics Resources 6.7 where KEGG pathway analysis was applied to uncover enriched functional pathways. Objects highlighted in red represent down-regulated gene products. Green boxes correspond to gene products specific for the Bos Taurus genome that were unaltered by treatments. Significance of gene-pathway enrichment conducted with a modified Fisher's exact test (EASE score, $P<0.001$ ). 


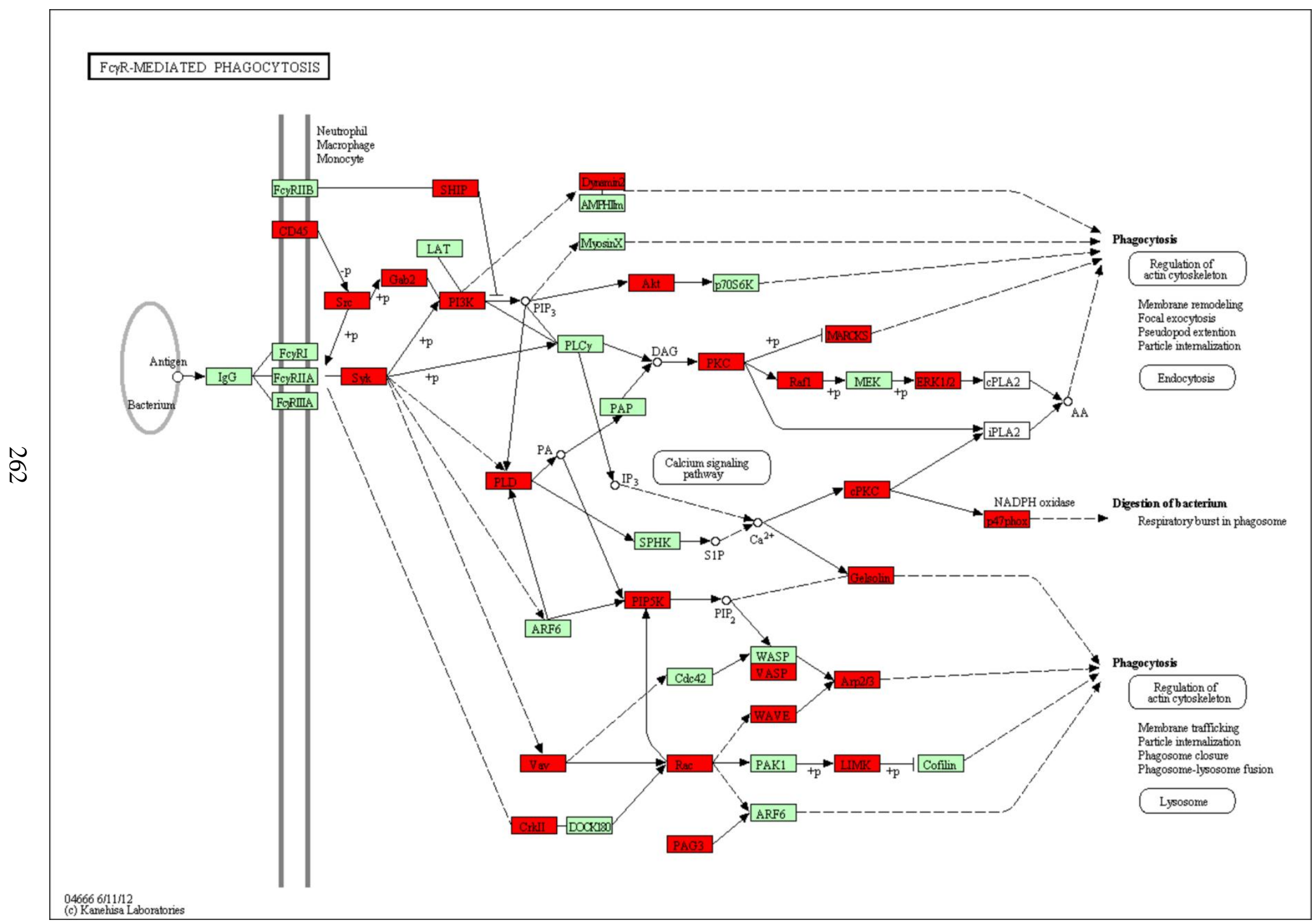


Enriched GO terms related to biological processes and molecular functions for the genes differentially regulated for the comparisons between trace mineral supplementation groups are listed in Table 6.4. A total of 49 annotation terms were enriched for the genes differentially regulated by organic trace mineral supplementation relative to the inorganic group (Table 6.5, $P<0.100$ ). No enriched GO terms were detected for the remaining contrasts of dietary supplementation $(P>0.100)$. The top five terms enriched in the comparison of organic and inorganic trace mineral supplementation for non-activated PMNL ( $0 \mu \mathrm{g} / \mathrm{mL}$ of LPS) included purine nucleotide binding ( $\uparrow$ ), nucleotide binding ( $\uparrow$ ), purine ribonucleotide binding $(\uparrow)$, ribonucleotide binding $(\uparrow)$, and vesicle-mediated transport $(\downarrow)$. In addition, several GO terms related to immunity were identified such as immune response $(\uparrow)$, response to virus $(\uparrow)$, endocytosis $(\downarrow)$, and response to fungus ( $($; Table 6.5A, $P<0.100)$. Furthermore, the top five terms enriched in the comparison of organic and inorganic trace mineral supplementation for LPS-activated PMNL (50 $\mu \mathrm{g} / \mathrm{mL}$ of LPS) were RNA binding ( $\downarrow$ ), ISG15-protein conjugation ( $\uparrow$ ), response to virus $(\uparrow)$, ds RNA adenosine deaminase activity $(\downarrow)$, and adenosine deaminase activity ( $\downarrow$; Table 6.5B, $P<0.100)$. 
Table 6.5. Enriched annotation terms for genes differentially regulated due to trace mineral supplementation from organic sources (relative to inorganic sources) in neutrophils (PMNL) treated with 0 or $50 \mu \mathrm{g} / \mathrm{mL}$ of Escherichia coli lipopolysaccharide (LPS)

\begin{tabular}{lcccc}
\hline Term & Class $^{1}$ & $\uparrow$ or $\downarrow$ & Genes & P value $^{2}$ \\
\hline A. Non-activated PMNL $(0 \mu g / m l$ of LPS $)$ & & & & \\
Purine nucleotide binding & & & & \\
Nucleotide binding & MF & $\uparrow$ & 14 & 0.009 \\
Purine ribonucleotide binding & MF & $\uparrow$ & 14 & 0.033 \\
Ribonucleotide binding & MF & $\uparrow$ & 13 & 0.016 \\
Vesicle-mediated transport & MF & $\uparrow$ & 13 & 0.016 \\
Protein transport & BP & $\downarrow$ & 10 & 0.000 \\
Establishment of protein localization & BP & $\downarrow$ & 10 & 0.001 \\
Protein localization & BP & $\downarrow$ & 10 & 0.001 \\
Positive regulation of molecular function & BP & $\downarrow$ & 10 & 0.002 \\
Intracellular transport & BP & $\downarrow$ & 6 & 0.005 \\
Gtpase activity & BP & $\downarrow$ & 6 & 0.031 \\
Positive regulation of catalytic activity & MF & $\uparrow$ & 5 & 0.001 \\
Immune response & BP & $\downarrow$ & 5 & 0.014 \\
Intracellular protein transport & BP & $\uparrow$ & 5 & 0.023 \\
Cellular protein localization & BP & $\downarrow$ & 5 & 0.036 \\
Cellular macromolecule localization & BP & $\downarrow$ & 5 & 0.044 \\
GTP binding & BP & $\downarrow$ & 5 & 0.045 \\
Guanyl ribonucleotide binding & MF & $\uparrow$ & 5 & 0.046 \\
Guanyl nucleotide binding & MF & $\uparrow$ & 5 & 0.050 \\
RNA binding & MF & $\uparrow$ & 5 & 0.051 \\
Response to virus & MF & $\uparrow$ & 5 & 0.080 \\
Endocytosis & BP & $\uparrow$ & 4 & 0.000 \\
Membrane invagination & BP & $\downarrow$ & 3 & 0.086 \\
Steroid metabolic process & BP & $\downarrow$ & 3 & 0.086 \\
Ds RNA adenosine deaminase activity & BP & $\downarrow$ & 3 & 0.097 \\
ISG15-protein conjugation & MF & $\uparrow$ & 2 & 0.010 \\
Adenosine deaminase activity & BP & $\uparrow$ & 2 & 0.015 \\
Urea metabolic process & MF & $\uparrow$ & 2 & 0.028 \\
Urea cycle & BP & $\downarrow$ & 2 & 0.031 \\
Amide biosynthetic process & BP & $\downarrow$ & 2 & 0.031 \\
Intermediate filament organization & BP & $\downarrow$ & 2 & 0.037 \\
Arginine metabolic process & BP & $\downarrow$ & 2 & 0.037 \\
Filament cytoskeleton organization & BP & $\downarrow$ & 2 & 0.049 \\
& BP & $\downarrow$ & 2 & 0.055 \\
\hline
\end{tabular}


Table 6.5 (continued)

\begin{tabular}{lcccc} 
Term & Class $^{1}$ & $\uparrow$ or $\downarrow$ & Genes & P value $^{2}$ \\
\hline Detection of biotic stimulus & BP & $\downarrow$ & 2 & 0.061 \\
Deaminase activity & MF & $\uparrow$ & 2 & 0.065 \\
Intermediate filament-based process & BP & $\downarrow$ & 2 & 0.073 \\
Protein amino acid ADP-ribosylation & BP & $\downarrow$ & 2 & 0.073 \\
Hydrolase activity, acting on C-N & MF & $\uparrow$ & 2 & 0.074 \\
Response to fungus & BP & $\downarrow$ & 2 & 0.090
\end{tabular}

\section{B. LPS-activated PMNL (50 $\mu \mathrm{g} / \mathrm{ml}$ of LPS)}

RNA binding

ISG15-protein conjugation

Response to virus

Ds RNA adenosine deaminase activity

Adenosine deaminase activity

Deaminase activity

Hydrolase activity, acting on C-N

Double-stranded RNA binding

Purine NTP-dependent helicase activity

ATP-dependent helicase activity

${ }^{1} \mathrm{BP}=$ Biological process, MF = Molecular function.

\begin{tabular}{llll} 
MF & $\downarrow$ & 4 & 0.014 \\
BP & $\uparrow$ & 2 & 0.005 \\
BP & $\uparrow$ & 2 & 0.036 \\
MF & $\downarrow$ & 2 & 0.003 \\
MF & $\downarrow$ & 2 & 0.009 \\
MF & $\downarrow$ & 2 & 0.021 \\
MF & $\downarrow$ & 2 & 0.024 \\
MF & $\downarrow$ & 2 & 0.034 \\
MF & $\downarrow$ & 2 & 0.089 \\
MF & $\downarrow$ & 2 & 0.089 \\
\hline
\end{tabular}

${ }^{2}$ Significance of gene-term enrichment test conducted with a modified Fisher's exact test (EASE score). 


\section{DISCUSSION}

In the current study, an average of 24.7 million 50-base pair reads per cDNA library was generated. Approximately 21.6 million of these reads passed the quality filters, and 18.9 million reads mapped to the bovine genome using gene sequences as the alignment segments. Usually, a high percentage of sequenced fragments are expected to map to known and predicted exons when RNA-seq is conducted on poly(A) ${ }^{+}$RNA (Mortazavi et al., 2008). These investigators, for example, reported that $93 \%$ of reads obtained from poly $(\mathrm{A})^{+}$RNA purified from mouse liver mapped to the source genome. In our experiments, $88 \%$ of filtered reads successfully mapped to the bovine genome. We evaluated the differential expression between our experimental conditions using digital counts of these reads. Our contrast analysis approach revealed that, regardless of source, only a few genes were altered due to trace mineral supplementation when compared with the basal group. As a result no enriched pathways or GO terms were detected for these contrasts. Interestingly, one of the transcripts up-regulated in LPS-activated PMNL by both inorganic and organic trace mineral supplementation (vs. the basal diet) was the gene encoding for GRB2-related adaptor protein 2 (GRAP2). This protein interacts with

other intracellular molecules during leukocyte-specific protein-tyrosine kinase signaling (Tanase, 2010). Although the role of GRAP2 in neutrophils is less clear, the protein encoded by this gene is required for the proliferation and immune response of CD8+ T cells in mice (Zhang et al., 2011). Hence, our finding suggests that trace mineral supplementation (regardless of source) may influence the intracellular signaling that leads to leukocyte proliferation. However, interpretation of changes in the expression of 
individual genes should be interpreted cautiously because of the small number of detected transcripts in these contrasts and a false discovery rate of $20 \%$.

That a limited number of genes were altered by feeding either inorganic or organic trace minerals (vs. basal) suggests minimal changes in PMNL gene expression when dietary trace mineral intake is suboptimal. In a complementary study by our group, PMNL from cows receiving no added trace minerals had a slight decrease in phagocytosis of E. coli particles, compared with animals supplemented inorganic trace minerals (Revelo et al., dissertation chapter five). However, PMNL from nonsupplemented cows did not show impaired ROS production, release of NETs, chemotaxis, or killing of E. coli (Revelo et al., dissertation chapter five). Together with our analysis of gene expression, these results suggest that the non-supplemented (for approximately 2 months) cows only developed a marginal trace mineral deficiency without substantial effects on PMNL. As suggested by Suttle and Jones (1989), dairy cows may prioritize the routing of trace minerals towards immune function over processes less critical for survival when the supply of these nutrients is limited. In contrast, supplementation of cows with organic trace minerals had a strong effect on PMNL gene expression, at least when compared with inorganic trace minerals. Organic trace mineral supplementation (vs. inorganic) altered the expression of 230 and 47 transcripts in non-activated and LPS-activated PMNL, respectively. Functional annotation analyses revealed that organic trace mineral supplementation (vs. inorganic) up-regulated three pathways relevant to the innate immune response: the RIG-I-like receptor signaling (non-activated and LPS-activated PMNL), cytosolic DNA-sensing (non-activated and LPS-activated PMNL), and TLR signaling pathway (non-activated 
PMNL). Membrane-bound TLRs are a subtype of PRRs used by phagocytes and other antigen presenting cells (APC) to recognize pathogen-associated molecular patterns (Werling et al., 2006). Similarly, the RIG-I-like receptors (RLRs) are PPRs involved in the recognition of viral nucleic acids in the cytoplasm. This recognition of viral infection by RLRs results in downstream signaling pathways leading to the transcription of proinflammatory cytokines (Kawai and Akira, 2008). In addition, recent studies have identified a novel cytosolic DNA-sensing pathway in which cytosolic RNA polymerase III recognizes and transcribes microbial DNA into 5'-triphosphate dsRNA that subsequently triggers the RIG-I-like receptor signaling pathway (Cao, 2009). The findings in the current study suggest that organic trace mineral supplementation may enhance the ability of the PMNL to detect the presence of infection and induce an immune response, at least at the gene expression level.

Our results suggest that increased bioavailability of $\mathrm{Cu}, \mathrm{Zn}, \mathrm{Co}$, and $\mathrm{Mn}$ in cows fed trace minerals from organic sources may improve PMNL performance via changes in gene expression, at least compared with inorganic sources. Several experiments with human cell lines and rodents have indicated that deficiencies in trace minerals alter the expression of immune-related genes. For instance, incubation of a monocyte-macrophage cell line in medium devoid of $\mathrm{Zn}$ decreased the mRNA contents of TNF, IL- $\beta 1$ and IL-8 suggesting that $\mathrm{Zn}$ is needed for cytokine synthesis (Bao et al., 2003). Similarly, the same investigators reported that $\mathrm{Zn}$-deficient $\mathrm{T}$-cells have decreased mRNA transcripts encoding for INF- $\gamma$, IL-12Rß2, and T-box 21, which are essential for T-cell differentiation (Bao et al., 2011). In mice, a marginal dietary Zn deficiency resulted in altered expression of 230 genes in thymic tissue including transcripts encoding for 
proteins involved in thymic development (Moore et al., 2001). One mechanism by which trace minerals may regulate gene expression is through changes in redox signaling. Trace minerals such as $\mathrm{Mn}, \mathrm{Cu}, \mathrm{Co}$, and $\mathrm{Zn}$ can function either as direct scavengers of free radicals or as components of enzymes involved in the antioxidant defense system including the superoxide dismutases or SODs (Spears and Weiss, 2008). Three isoforms of SODs exist in mammals: the cytoplasmic $\mathrm{Cu}-\mathrm{Zn}$ SOD (SOD1), the mitochondrial Mn SOD (SOD2), and the extracellular Cu-Zn SOD (SOD3). All three of these enzymes require $\mathrm{Cu}$ and $\mathrm{Zn}$ for their activation and catalyze the dismutation of $\mathrm{O}_{2}{ }^{-}$to $\mathrm{H}_{2} \mathrm{O}_{2}$ (Fukai and Ushio-Fukai, 2011). Because intracellular $\mathrm{O}_{2}{ }^{-}$and $\mathrm{H}_{2} \mathrm{O}_{2}$ can directly activate the transcription factors nuclear factor- $\mathrm{kB}(\mathrm{NF}-\mathrm{\kappa B})$ and nuclear factor (erythroid-derived 2)like 2 (NRF2; Brigelius-Flohé and Flohé, 2011), the intracellular levels of these trace minerals may influence redox signaling and thus the expression of pro- or antiinflammatory genes. Furthermore, $\mathrm{Zn}$ can function as an independent intracellular molecule or as a component of $\mathrm{Zn}$-finger proteins and transcription factors (Murakami and Hirano, 2008). Zinc-finger proteins regulate the expression of growth factors and steroid receptors and require $\mathrm{Zn}$ to maintain the stability of their tertiary structure (Shankar and Prasad, 1998). Zinc-finger transcription factors may also have a critical role in the differentiation of neutrophils from myeloid progenitors. For example, mice lacking the Zn-finger transcription factor Gfi-1 have no mature neutrophils and are highly susceptible to abscess formation by gram-positive bacteria (Hock et al., 2003). Interestingly, several $\mathrm{Zn}$ finger proteins were up-regulated in PMNL from cows supplemented with organic trace minerals (vs. inorganic) in the present study. These included the Zn finger proteins 565 (ZNF565), 398 (ZNF398), and NFX1-type containing 
1 (ZNFX1), in addition to the ring finger proteins 38 (RNF38), and 213 (RNF213). Whether these changes in the expression of $\mathrm{Zn}$ transcription factors in response to organic trace mineral supplementation have an impact on PMNL development in dairy cows needs further investigation.

Dairy cows are more susceptible to mammary gland infections for several weeks around the time of parturition in part due to a compromised immune system (Mallard et al., 1998). Because the antimicrobial roles of PMNL provide the first line of defense against the invading pathogen, extensive research has focused on periparturient PMNL dysfunction (Paape et al., 2003). In the present study, the genetic profile of blood PMNL collected from prepartum (pretreatment, day -58 relative to parturition) and postpartum (day 6 relative to parturition) was examined. To avoid the bias of the different trace mineral supplementation treatments over time, only cows receiving trace mineral supplementation from inorganic sources (pretreatment dietary supplementation source) were included in this analysis as the postpartum group. The expression of a substantial number of genes was altered in non-activated (9589, $\uparrow 0$ and $\downarrow 9589)$ and LPS-activated (2001, $\uparrow 801$ and $\downarrow 1200)$ PMNL from postpartum, compared with prepartum cows. Remarkably, the number of genes with down-regulated expression greatly surpassed that of up-regulated genes, particularly in non-activated PMNL. Several pathways essential for the immune response were down-regulated in non-activated PMNL from postpartum, relative to prepartum cows. For example, the chemokine signaling, leukocyte transendothelial migration, Fc gamma R-mediated phagocytosis, cell adhesion molecules (CAMs), TLR signaling, lysosome, apoptosis, and NOD-like receptor signaling pathways were all down-regulated in non-activated PMNL from postpartum cows. These results 
provide an extensive amount of information on the alteration of gene expression that may explain the impaired performance of PMNL during the periparturient period. On the other hand, several interesting pathways were up-regulated in LPS-activated PMNL from postpartum (vs. prepartum) cows including glycolysis and fatty acid metabolism. The upregulation of glycolysis in LPS-activated PMNL suggests that these cells may use additional amounts of glucose as fuel during the postpartum period. During infection, glucose may be an important source of energy for PMNL because, upon activation, these cells increase their expression of the glucose transporters (GLUT), isoforms 1 and 3 (Maratou et al., 2007). In dairy cows, Waldron et al. (2003, 2006) reported increased plasma glucose concentration, hepatic gluconeogenesis, and glucose rate of appearance into blood following experimental immune activation. Similarly, Moyes et al. (2009) reported an increase in plasma glucose concentration after experimental intramammary infection with Streptococcus uberis.

Other researchers have investigated changes in global PMNL gene (Madsen et al., 2004; Burton et al., 2005) and protein (Lippolis et al., 2006a) expression during the periparturient period. Madsen et al. (2004) used microarrays containing 1,056 cDNA probes to identify fluctuations in gene expression between days $-7,0,0.25$, and 1 relative to parturition and discovered 42 genes with altered expression at calving. Furthermore, these investigators reported that genes encoding for pro-apoptotic proteins were strongly diminished at parturition whereas pro-survival genes were up-regulated. Although the timing of sample collection was different, these results are in agreement with our finding that PMNL collected from cows around the day of parturition (day 6 postpartum) have reduced apoptotic potential as evidenced by 22 down-regulated genes in this pathway. 
The same group later expanded their analysis of this dataset and reported that around parturition PMNL have altered expression of genes involved in the immune response such as cluster of differentiation (CD)62L, CD18, IL-8 receptor $\beta$, IкK alpha, and FcRN (Burton et al., 2005). At the protein level, Lippolis et al. (2006a) reported 24 proteins with down-regulated expression in PMNL collected on days 2 and 3 postpartum relative to PMNL from cows on day 28 prepartum. Proteins with down-regulated expression included the PMNL chemoattractant hepoxilin A3 and myeloperoxidase (MPO) involved in ROS generation. Consistently, the gene expression of MPO was 59.6\% down-regulated in LPS-activated PMNL from postpartum cows in the present study. In addition, several groups including our own laboratory have previously reported altered expression of individual genes involved in cytokine synthesis (Revelo and Waldron, 2012), and TLR signaling (Stevens et al., 2011). These reports are in agreement with our finding that the chemokine and TLR signaling pathways are down-regulated in non-activated PMNL from postpartum cows. In summary, the use of high-throughput sequencing technology in the current study provided an unprecedented ability to discover an extensive collection of genes with altered expression in PMNL from postpartum cows. Using these differentially regulated genes, functional annotation analysis revealed pathways that increase our biological insights into periparturient bovine immune-alteration and highlight opportunities for interventions.

\section{CONCLUSIONS}

Regardless of source, minimal effects of trace mineral supplementation on the

global gene expression profile of non-activated and LPS-activated PMNL were detected 
when compared with a basal diet containing none of these added trace minerals. This finding suggests that non-supplemented cows developed only a marginal dietary deficiency for these nutrients and may have used homeorhetic mechanisms to prioritize trace minerals towards immune function. In contrast, organic trace mineral supplementation, compared with that from inorganic sources, altered the expression of 230 and 47 transcripts in non-activated and LPS-activated PMNL, respectively. Pathways up-regulated by organic, relative to inorganic trace minerals, included the RIG-I-like receptor signaling (non-activated and LPS-activated PMNL), cytosolic DNA-sensing (non-activated and LPS-activated PMNL), and TLR signaling pathway (non-activated PMNL). These results suggest that organic trace minerals (vs. inorganic) stimulate PMNL pathways involved in antigen recognition and immune response. In addition, PMNL collected from cows on day 6 postpartum had altered expression of genes towards defective immune function, compared with those harvested from cows on day -58 relative to parturition. Relative to PMNL from prepartum cows, PMNL collected from postpartum animals had altered expression of 9589 and 2001 transcripts in non-activated and LPS-activated PMNL, respectively. Functional annotation analysis indicated that the chemokine signaling, leukocyte transendothelial migration, Fc gamma R-mediated phagocytosis, cell adhesion molecules (CAMs), TLR signaling, lysosome, apoptosis, and NOD-like receptor signaling pathways were down-regulated in PMNL from postpartum (vs. prepartum) cows. The use of RNA-sequencing allowed the discovery of wide assortment of genes with altered expression that may explain defective PMNL function in the periparturient period of dairy cows. 
Table 6.6. Supplemental. Differential expression of genes in neutrophils (PMNL) collected from cows fed no added dietary trace minerals $(n=7)$ or supplemented with trace minerals from either inorganic $(n=7)$ or organic $(n=7)$ sources after incubation with 0 or $50 \mu \mathrm{g} / \mathrm{mL}$ of $E$. coli lipopolysaccharide for $120 \mathrm{~min}$

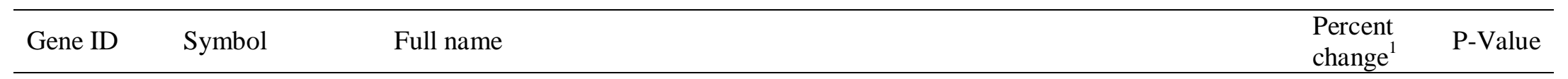

A. Genes differentially expressed in PMNL from cows fed inorganic trace minerals relative to those fed the basal diet ${ }^{2}$

Non-activated PMNL $(0 \mu \mathrm{g} / \mathrm{ml}$ E. Coli LPS $)$

\begin{tabular}{|c|c|c|c|c|}
\hline$\underline{514979}$ & CLEC6A & C-type lectin domain family 6 , member A & $127.3 \uparrow$ & $<0.001$ \\
\hline$\underline{751808}$ & WC-7 & WC1 isolate $\mathrm{CH} 149$ & $88.4 \uparrow$ & $<0.001$ \\
\hline$\underline{504509}$ & ZAP70 & Zeta-chain (TCR) associated protein kinase 70kda & $74.5 \uparrow$ & $<0.001$ \\
\hline$\underline{617342}$ & CCDC83 & Coiled-coil domain containing 83 & $63.2 \uparrow$ & $<0.001$ \\
\hline 751809 & WC1 & WC1 isolate DV9 & $54 \uparrow$ & $<0.001$ \\
\hline 404104 & DMBT1 & Deleted in malignant brain tumors 1 & $49.9 \downarrow$ & $<0.001$ \\
\hline
\end{tabular}

$\stackrel{1}{N}$

LPS-activated PMNL $(50 \mu \mathrm{g} / \mathrm{ml}$ E. Coli LPS $)$

\begin{tabular}{|c|c|c|c|c|}
\hline$\underline{514979}$ & CLEC6A & C-type lectin domain family 6 , member A & $174.6 \uparrow$ & $<0.001$ \\
\hline$\underline{751808}$ & WC-7 & WC1 isolate $\mathrm{CH} 149$ & $86.8 \uparrow$ & $<0.001$ \\
\hline$\underline{504509}$ & ZAP70 & Zeta-chain (TCR) associated protein kinase 70kda & $80.9 \uparrow$ & $<0.001$ \\
\hline$\underline{522346}$ & GRAP2 & GRB2-related adaptor protein 2 & $59.9 \uparrow$ & $<0.001$ \\
\hline$\underline{617342}$ & $\mathrm{CCDC} 83$ & Coiled-coil domain containing 83 & $57.4 \uparrow$ & $<0.001$ \\
\hline 751809 & WC1 & WC1 isolate DV9 & $50.2 \uparrow$ & $<0.001$ \\
\hline
\end{tabular}

B. Genes differentially expressed in PMNL from cows fed organic trace minerals relative to those fed the basal diet ${ }^{2}$

Non-activated PMNL $(0 \mu \mathrm{g} / \mathrm{ml}$ E. Coli LPS $)$

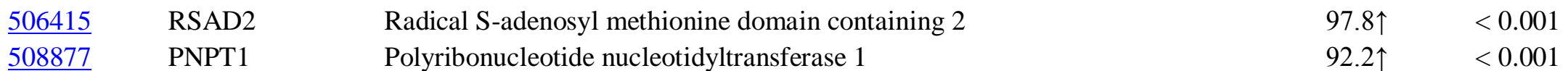


Table 6.6. Supplemental (continued)

\begin{tabular}{|c|c|c|c|c|}
\hline Gene ID & Symbol & Full name & $\begin{array}{l}\text { Percent } \\
\text { change }^{1}\end{array}$ & P-Value \\
\hline 281747 & EPYC & Epiphycan & $30.5 \downarrow$ & $<0.001$ \\
\hline$\overline{505407}$ & IGHE & Immunoglobulin heavy constant epsilon & $88.5 \downarrow$ & $<0.001$ \\
\hline$\underline{100337466}$ & LOC100337466 & Mucin-like & $81.7 \downarrow$ & $<0.001$ \\
\hline$\underline{505653}$ & MS4A1 & Membrane-spanning 4-domains, subfamily A, member 1 & $79.6 \downarrow$ & $<0.001$ \\
\hline$\underline{404104}$ & DMBT1 & Deleted in malignant brain tumors 1 & $55 \downarrow$ & $<0.001$ \\
\hline
\end{tabular}

LPS-activated PMNL (50 $\mu \mathrm{g} / \mathrm{ml}$ E. Coli LPS)

\begin{tabular}{|c|c|c|c|c|}
\hline$\underline{506415}$ & RSAD2 & Radical S-adenosyl methionine domain containing 2 & $95.1 \uparrow$ & $<0.001$ \\
\hline$\underline{513185}$ & PARP12 & Poly (ADP-ribose) polymerase family, member 12 & $74.5 \uparrow$ & $<0.001$ \\
\hline$\underline{522346}$ & GRAP2 & GRB2-related adaptor protein 2 & $70.8 \uparrow$ & $<0.001$ \\
\hline 513479 & RNF114 & Ring finger protein 114 & $68.2 \uparrow$ & $<0.001$ \\
\hline
\end{tabular}

Ư

C. Genes differentially expressed in PMNL from cows fed organic relative to those fed inorganic trace minerals ${ }^{2}$

Non-activated PMNL $(0 \mu \mathrm{g} / \mathrm{ml}$ E. Coli LPS $)$

\begin{tabular}{|c|c|c|c|c|}
\hline$\underline{530757}$ & SULT6B1 & Sulfotransferase family, cytosolic, 6B, member 1 & $421.4 \uparrow$ & $<0.001$ \\
\hline$\underline{613907}$ & BHLHE41 & Basic helix-loop-helix family, member e41 & $387.8 \uparrow$ & 0.002 \\
\hline$\underline{509859}$ & TRANK1 & Tetratricopeptide repeat and ankyrin repeat containing 1 & $242.4 \uparrow$ & 0.001 \\
\hline$\underline{791010}$ & MIR142 & Microrna mir-142 & $202.6 \uparrow$ & 0.002 \\
\hline$\underline{508333}$ & ZBP1 & Z-DNA binding protein 1 & $200.3 \uparrow$ & $<0.001$ \\
\hline$\underline{281871}$ & ISG15 & ISG15 ubiquitin-like modifier & $192.7 \uparrow$ & 0.001 \\
\hline$\underline{280873}$ & MX2 & Myxovirus (influenza virus) resistance 2 (mouse) & $189 \uparrow$ & 0.001 \\
\hline$\underline{540438}$ & STOML1 & Stomatin (EPB72)-like 1 & $174.4 \uparrow$ & $<0.001$ \\
\hline$\underline{506415}$ & RSAD2 & Radical S-adenosyl methionine domain containing 2 & $161.5 \uparrow$ & $<0.001$ \\
\hline$\underline{512913}$ & IFI6 & Interferon, alpha-inducible protein 6 & $160.1 \uparrow$ & 0.001 \\
\hline
\end{tabular}


Table 6.6. Supplemental (continued)

\begin{tabular}{|c|c|c|c|c|}
\hline Gene ID & Symbol & Full name & $\begin{array}{l}\text { Percent } \\
\text { change }^{1}\end{array}$ & P-Value \\
\hline$\underline{615827}$ & CKS1B & CDC28 protein kinase regulatory subunit $1 \mathrm{~B}$ & $158.6 \uparrow$ & $<0.001$ \\
\hline$\overline{529660}$ & OAS2 & 2'-5'-oligoadenylate synthetase $2,69 / 71 \mathrm{kda}$ & $151.2 \uparrow$ & $<0.001$ \\
\hline$\overline{525795}$ & AGRN & Agrin & $141.9 \uparrow$ & 0.002 \\
\hline$\overline{535490}$ & IFIH1 & Interferon induced with helicase $\mathrm{C}$ domain 1 & $132.8 \uparrow$ & $<0.001$ \\
\hline$\underline{614838}$ & PIK3R5 & Phosphoinositide-3-kinase, regulatory subunit 5 & $129.6 \uparrow$ & 0.002 \\
\hline 100139892 & HPGDS & Hematopoietic prostaglandin D synthase & $128.1 \uparrow$ & $<0.001$ \\
\hline$\underline{785309}$ & LOC785309 & T-cell receptor alpha chain V region PY14-like & $127.5 \uparrow$ & $<0.001$ \\
\hline$\underline{538504}$ & ZNF565 & Zinc finger protein 565 & $122.7 \uparrow$ & $<0.001$ \\
\hline$\underline{525559}$ & ZNF398 & Zinc finger protein 398 & $120.6 \uparrow$ & 0.003 \\
\hline$\underline{509866}$ & HIVEP3 & Human immunodeficiency virus type I enhancer binding protein 3 & $117.7 \uparrow$ & 0.001 \\
\hline$\underline{508877}$ & PNPT1 & Polyribonucleotide nucleotidyltransferase 1 & $114.6 \uparrow$ & $<0.001$ \\
\hline$\underline{790428}$ & LOC790428 & Gag-like & $113.6 \uparrow$ & 0.001 \\
\hline$\overline{509283}$ & RNF213 & Ring finger protein 213 & $112.5 \uparrow$ & $<0.001$ \\
\hline$\underline{505134}$ & ADAR & Adenosine deaminase, RNA-specific & $111.3 \uparrow$ & $<0.001$ \\
\hline$\underline{347700}$ & EIF2AK2 & Eukaryotic translation initiation factor 2-alpha kinase 2 & $110.3 \uparrow$ & $<0.001$ \\
\hline$\underline{508378}$ & DHX58 & DEXH (Asp-Glu-X-His) box polypeptide 58 & $106.5 \uparrow$ & $<0.001$ \\
\hline$\underline{514701}$ & FAM3B & Family with sequence similarity 3 , member B & $103.5 \uparrow$ & $<0.001$ \\
\hline$\underline{100138545}$ & PML & Promyelocytic leukemia & $103.2 \uparrow$ & $<0.001$ \\
\hline$\underline{523809}$ & UBASH3B & Ubiquitin associated and $\mathrm{SH} 3$ domain containing B & $99.7 \uparrow$ & $<0.001$ \\
\hline$\underline{527577}$ & TRIM4 & Tripartite motif containing 4 & $97.1 \uparrow$ & 0.002 \\
\hline$\underline{613856}$ & LY86 & Lymphocyte antigen 86 & $93.9 \uparrow$ & 0.002 \\
\hline$\underline{529500}$ & AHRR & Aryl-hydrocarbon receptor repressor & $92.4 \uparrow$ & 0.001 \\
\hline$\underline{504760}$ & DDX58 & DEAD (Asp-Glu-Ala-Asp) box polypeptide 58 & $89.7 \uparrow$ & $<0.001$ \\
\hline$\underline{529115}$ & HIVEP1 & Human immunodeficiency virus type I enhancer binding protein 1 & $89.5 \uparrow$ & 0.001 \\
\hline$\underline{509620}$ & FTSJD2 & Ftsj methyltransferase domain containing 2 & $89.2 \uparrow$ & $<0.001$ \\
\hline$\underline{510923}$ & TRIM25 & Tripartite motif containing 25 & $84.9 \uparrow$ & 0.002 \\
\hline$\underline{513185}$ & PARP12 & Poly (ADP-ribose) polymerase family, member 12 & $84.7 \uparrow$ & $<0.001$ \\
\hline
\end{tabular}


Table 6.6. Supplemental (continued)

\begin{tabular}{|c|c|c|c|c|}
\hline Gene ID & Symbol & Full name & $\begin{array}{l}\text { Percent } \\
\text { change }^{1}\end{array}$ & P-Value \\
\hline$\underline{515202}$ & USP18 & Ubiquitin specific peptidase 18 & $84.7 \uparrow$ & $<0.001$ \\
\hline$\overline{516599}$ & LOC516599 & Tripartite motif containing 5-like & $83.5 \uparrow$ & $<0.001$ \\
\hline$\overline{539807}$ & ZNFX1 & Zinc finger, NFX1-type containing 1 & $83.5 \uparrow$ & 0.001 \\
\hline$\overline{514492}$ & TRIM6 & Tripartite motif containing 6 & $81.1 \uparrow$ & $<0.001$ \\
\hline$\underline{506566}$ & ALG5 & Asparagine-linked glycosylation 5, dolichyl-phosphate beta-glucosyltransferase & $79.2 \uparrow$ & 0.001 \\
\hline$\underline{280872}$ & MX1 & Myxovirus (influenza virus) resistance 1 , interferon-inducible protein p78 & $78.1 \uparrow$ & 0.002 \\
\hline$\underline{516949}$ & GBP5 & Guanylate binding protein 5 & $74.4 \uparrow$ & 0.001 \\
\hline$\underline{613533}$ & FLNB & Filamin B, beta & $72.2 \uparrow$ & $<0.001$ \\
\hline$\underline{541459}$ & $\mathrm{KCNC} 1$ & Potassium voltage-gated channel, Shaw-related subfamily, member 1 & $63.7 \uparrow$ & $<0.001$ \\
\hline$\underline{532026}$ & CALHM1 & Calcium homeostasis modulator 1 & $63.7 \uparrow$ & 0.003 \\
\hline$\underline{510814}$ & STAT1 & Signal transducer and activator of transcription $1,91 \mathrm{kda}$ & $63 \uparrow$ & 0.002 \\
\hline$\underline{782527}$ & LOC782527 & Tripartite motif protein 5 alpha-like & $62.2 \uparrow$ & $<0.001$ \\
\hline$\overline{507007}$ & NUBP1 & Nucleotide binding protein 1 & $60.8 \uparrow$ & 0.001 \\
\hline$\underline{509643}$ & PROSC & Proline synthetase co-transcribed homolog (bacterial) & $59.9 \uparrow$ & 0.002 \\
\hline$\underline{539884}$ & RFX5 & Regulatory factor X, 5 (influences HLA class II expression) & $58.6 \uparrow$ & $<0.001$ \\
\hline$\underline{514143}$ & LOC514143 & Uncharacterized LOC514143 & $58.6 \uparrow$ & 0.001 \\
\hline$\underline{511723}$ & RPP38 & Ribonuclease P/MRP 38kda subunit & $58.4 \uparrow$ & 0.002 \\
\hline$\underline{100337081}$ & LOC 100337081 & Egf-like module containing, mucin-like, hormone receptor-like 3-like & $58 \uparrow$ & 0.003 \\
\hline$\underline{515333}$ & $\mathrm{ABCC} 4$ & ATP-binding cassette, sub-family C (CFTR/MRP), member 4 & $57.9 \uparrow$ & $<0.001$ \\
\hline$\underline{519920}$ & EIF4E2 & Eukaryotic translation initiation factor $4 \mathrm{E}$ family member 2 & $57 \uparrow$ & $<0.001$ \\
\hline$\underline{789894}$ & PAPD5 & PAP associated domain containing 5 & $54.1 \uparrow$ & 0.001 \\
\hline$\underline{527520}$ & HERC6 & Hect domain and RLD 6 & $54.1 \uparrow$ & 0.002 \\
\hline$\underline{533664}$ & AP1AR & Adaptor-related protein complex 1 associated regulatory protein & $51 \uparrow$ & $<0.001$ \\
\hline$\underline{509003}$ & NUCB2 & Nucleobindin 2 & $49.1 \uparrow$ & 0.001 \\
\hline$\underline{539153}$ & SLC16A14 & Solute carrier family 16, member 14 (monocarboxylic acid transporter 14 ) & $48.9 \uparrow$ & $<0.001$ \\
\hline$\underline{786435}$ & LOC786435 & RING finger protein 38-like & $46.4 \uparrow$ & 0.002 \\
\hline$\underline{508347}$ & LOC508347 & Interferon-induced protein 44-like & $45.3 \uparrow$ & 0.002 \\
\hline
\end{tabular}


Table 6.6. Supplemental (continued)

\begin{tabular}{|c|c|c|c|c|}
\hline Gene ID & Symbol & Full name & $\begin{array}{l}\text { Percent } \\
\text { change }^{1}\end{array}$ & P-Value \\
\hline$\underline{532877}$ & RNF38 & Ring finger protein 38 & $44.8 \uparrow$ & 0.002 \\
\hline$\overline{521855}$ & MTSS1L & Metastasis suppressor 1-like & $43.4 \uparrow$ & 0.003 \\
\hline$\overline{525806}$ & PROSER1 & Proline and serine rich 1 & $41.6 \uparrow$ & $<0.001$ \\
\hline$\underline{100299991}$ & LOC100299991 & Uncharacterized LOC100299991 & $39.6 \uparrow$ & 0.003 \\
\hline 784219 & NUP214 & Nucleoporin $214 \mathrm{kda}$ & $39.3 \uparrow$ & $<0.001$ \\
\hline$\underline{533289}$ & PIP4K2A & Phosphatidylinositol-5-phosphate 4-kinase, type II, alpha & $38.6 \uparrow$ & $<0.001$ \\
\hline$\underline{520327}$ & KYNU & Kynureninase & $38.6 \uparrow$ & 0.001 \\
\hline$\underline{782145}$ & LOC782145 & Hcg1648656-like & $38.1 \uparrow$ & 0.003 \\
\hline$\underline{539350}$ & TICAM2 & Toll-like receptor adaptor molecule 2 & $37.7 \uparrow$ & 0.002 \\
\hline$\underline{541014}$ & KIAA1143 & KIAA1143 ortholog & $36.8 \uparrow$ & $<0.001$ \\
\hline$\underline{507204}$ & NLK & Nemo-like kinase & $36 \uparrow$ & 0.002 \\
\hline$\underline{511298}$ & NUB1 & Negative regulator of ubiquitin-like proteins 1 & $35.3 \uparrow$ & 0.002 \\
\hline$\underline{100125937}$ & SNX22 & Sorting nexin 22 & $33.8 \uparrow$ & 0.001 \\
\hline$\underline{507792}$ & GTF2IRD1 & GTF2I repeat domain containing 1 & $33.7 \uparrow$ & 0.001 \\
\hline$\underline{532560}$ & SLC25A15 & Solute carrier family 25 (mitochondrial carrier; ornithine transporter) member 15 & $33.6 \uparrow$ & 0.001 \\
\hline$\underline{533910}$ & DLD & Dihydrolipoamide dehydrogenase & $32.4 \uparrow$ & 0.002 \\
\hline$\underline{540514}$ & ARSJ & Arylsulfatase family, member $\mathbf{J}$ & $31.7 \uparrow$ & 0.001 \\
\hline$\underline{538618}$ & ENDOD1 & Endonuclease domain containing 1 & $31.4 \uparrow$ & 0.002 \\
\hline$\underline{617066}$ & LYPD4 & LY6/PLAUR domain containing 4 & $30.4 \uparrow$ & 0.001 \\
\hline$\underline{506539}$ & SIK3 & SIK family kinase 3 & $29 \uparrow$ & 0.001 \\
\hline$\underline{781710}$ & LOC781710 & Uncharacterized LOC781710 & $29 \uparrow$ & 0.002 \\
\hline$\underline{507486}$ & SHQ1 & SHQ1 homolog (S. Cerevisiae) & $28.8 \uparrow$ & 0.003 \\
\hline$\underline{530939}$ & IGSF3 & Immunoglobulin superfamily, member 3 & $26.7 \uparrow$ & 0.002 \\
\hline$\underline{527807}$ & FBXO42 & F-box protein 42 & $25.8 \uparrow$ & 0.001 \\
\hline$\underline{618087}$ & LOC618087 & Cd46 molecule, complement regulatory protein-like & $20.5 \uparrow$ & 0.002 \\
\hline 281889 & KRT17 & Keratin 17 & $87.9 \downarrow$ & $<0.001$ \\
\hline$\underline{505407}$ & IGHE & Immunoglobulin heavy constant epsilon & $86 \downarrow$ & $<0.001$ \\
\hline
\end{tabular}


Table 6.6. Supplemental (continued)

\begin{tabular}{|c|c|c|c|c|}
\hline Gene ID & Symbol & Full name & $\begin{array}{l}\text { Percent } \\
\text { change }^{1}\end{array}$ & P-Value \\
\hline$\underline{282305}$ & PGLYRP1 & Peptidoglycan recognition protein 1 & $85.4 \downarrow$ & $<0.001$ \\
\hline$\overline{404111}$ & KRT14 & Keratin 14 & $81.9 \downarrow$ & $<0.001$ \\
\hline$\overline{505653}$ & MS4A1 & Membrane-spanning 4-domains, subfamily A, member 1 & $81 \downarrow$ & $<0.001$ \\
\hline 100300028 & LOC 00300028 & Uncharacterized LOC100300028 & $80.3 \downarrow$ & 0.002 \\
\hline$\underline{509801}$ & CD79B & CD79b molecule, immunoglobulin-associated beta & $79.1 \downarrow$ & 0.001 \\
\hline$\underline{100300716}$ & LOC100300716 & Uncharacterized LOC100300716 & $75.8 \downarrow$ & 0.002 \\
\hline$\underline{100140137}$ & RNASE10 & Ribonuclease, rnase A family, 10 (non-active) & $72.7 \downarrow$ & 0.001 \\
\hline$\underline{100297100}$ & 100297100 & Ig heavy chain $\mathrm{C}$ region, membrane-bound form-like & $72.6 \downarrow$ & 0.001 \\
\hline$\underline{281270}$ & HOPX & HOP homeobox & $71.8 \downarrow$ & 0.002 \\
\hline$\underline{507948}$ & PTCH2 & Patched 2 & $71.7 \downarrow$ & 0.001 \\
\hline 100300424 & CAPNS2 & Calpain, small subunit 2 & $70.7 \downarrow$ & $<0.001$ \\
\hline$\underline{444876}$ & LOC444876 & $\operatorname{Igm}$ & $70.3 \downarrow$ & 0.002 \\
\hline$\underline{614288}$ & MKKS & Mckusick-Kaufman syndrome & $68.7 \downarrow$ & $<0.001$ \\
\hline$\underline{515777}$ & NOL3 & Nucleolar protein 3 (apoptosis repressor with CARD domain) & $67.3 \downarrow$ & 0.003 \\
\hline 100298507 & 100298507 & Uncharacterized LOC100298507 & $66.5 \downarrow$ & 0.003 \\
\hline$\underline{515013}$ & SCRN2 & Secernin 2 & $65.1 \downarrow$ & 0.002 \\
\hline$\underline{504950}$ & NPM3 & Nucleophosmin/nucleoplasmin 3 & $64.5 \downarrow$ & 0.002 \\
\hline$\underline{618664}$ & ART5 & ADP-ribosyltransferase 5 & $63.8 \downarrow$ & $<0.001$ \\
\hline$\underline{539375}$ & ACTN3 & Actinin, alpha 3 & $63.1 \downarrow$ & 0.001 \\
\hline$\underline{508623}$ & LOC508623 & MLN64 N-terminal homolog pseudogene & $62.7 \downarrow$ & $<0.001$ \\
\hline$\underline{280726}$ & ASS1 & Argininosuccinate synthase 1 & $62.3 \downarrow$ & 0.002 \\
\hline$\underline{100139105}$ & RAB3D & RAB3D, member RAS oncogene family & $61.6 \downarrow$ & $<0.001$ \\
\hline$\underline{505719}$ & FAM63A & Family with sequence similarity 63 , member A & $59.7 \downarrow$ & $<0.001$ \\
\hline$\underline{509956}$ & CTSG & Cathepsin $\mathrm{G}$ & $58.5 \downarrow$ & $<0.001$ \\
\hline$\underline{539101}$ & JAGN1 & Jagunal homolog 1 (Drosophila) & $58.5 \downarrow$ & $<0.001$ \\
\hline$\underline{337919}$ & MUC15 & Mucin 15 , cell surface associated & $58.1 \downarrow$ & 0.002 \\
\hline$\underline{506939}$ & CTTN & Cortactin & $57.7 \downarrow$ & 0.002 \\
\hline
\end{tabular}


Table 6.6. Supplemental (continued)

\begin{tabular}{|c|c|c|c|c|}
\hline Gene ID & Symbol & Full name & $\begin{array}{l}\text { Percent } \\
\text { change }^{1}\end{array}$ & P-Value \\
\hline 100294941 & C7H19orf38 & C7h19orf38 chromosome 7 open reading frame, human c19orf38 [ Bos taurus ] & $57.5 \downarrow$ & $<0.001$ \\
\hline$\overline{514978}$ & LOC514978 & Lipopolysaccharide-binding protein-like & $57.4 \downarrow$ & $<0.001$ \\
\hline$\underline{282523}$ & SLC25A11 & Solute carrier family 25 (mitochondrial carrier; oxoglutarate carrier), member 11 & $56.9 \downarrow$ & $<0.001$ \\
\hline$\underline{618550}$ & LOC618550 & Heme-binding protein 1 pseudogene & $56.8 \downarrow$ & 0.002 \\
\hline$\underline{618071}$ & LOC618071 & Uncharacterized LOC618071 & $56.7 \downarrow$ & $<0.001$ \\
\hline$\underline{534628}$ & NMRAL1 & Nmra-like family domain containing 1 & $56.7 \downarrow$ & $<0.001$ \\
\hline$\underline{534658}$ & WISP2 & WNT1 inducible signaling pathway protein 2 & $56.5 \downarrow$ & $<0.001$ \\
\hline$\underline{529919}$ & LOC529919 & Uncharacterized LOC529919 & $56 \downarrow$ & 0.001 \\
\hline$\underline{407145}$ & ART1 & ADP-ribosyltransferase 1 & $55.9 \downarrow$ & 0.001 \\
\hline$\underline{505016}$ & LAT2 & Linker for activation of $\mathrm{T}$ cells family, member 2 & $55 \downarrow$ & 0.002 \\
\hline$\underline{531514}$ & BMX & BMX non-receptor tyrosine kinase & $54.8 \downarrow$ & 0.002 \\
\hline$\underline{528055}$ & ANXA9 & Annexin A9 & $54.6 \downarrow$ & 0.001 \\
\hline$\underline{539171}$ & PDZD3 & PDZ domain containing 3 & $54.2 \downarrow$ & 0.002 \\
\hline 100336170 & 100336170 & Uncharacterized LOC100336170 & $54 \downarrow$ & 0.001 \\
\hline$\underline{534352}$ & MRPL18 & Mitochondrial ribosomal protein L18 & $53.8 \downarrow$ & 0.001 \\
\hline$\underline{516287}$ & CYB5R1 & Cytochrome b5 reductase 1 & $53.7 \downarrow$ & $<0.001$ \\
\hline$\underline{518201}$ & GLI4 & GLI family zinc finger 4 & $53.7 \downarrow$ & 0.002 \\
\hline$\underline{282846}$ & PYCARD & PYD and CARD domain containing & $53.4 \downarrow$ & 0.001 \\
\hline$\underline{535465}$ & IFI27L2 & Interferon, alpha-inducible protein 27-like 2 & $52.7 \downarrow$ & 0.002 \\
\hline$\underline{281388}$ & CNDP2 & CNDP dipeptidase 2 (metallopeptidase M20 family) & $52.4 \downarrow$ & 0.001 \\
\hline$\underline{783257}$ & ABCB6 & ATP-binding cassette, sub-family B (MDR/TAP), member 6 & $52.4 \downarrow$ & 0.002 \\
\hline$\underline{507309}$ & VAMP8 & Vesicle-associated membrane protein 8 (endobrevin) & $52.2 \downarrow$ & $<0.001$ \\
\hline$\underline{614423}$ & LOC614423 & Phosphoglycerate mutase 1-like & $52.1 \downarrow$ & 0.001 \\
\hline$\underline{281986}$ & PLCD1 & Phospholipase $\mathrm{C}$, delta 1 & $51.8 \downarrow$ & $<0.001$ \\
\hline$\underline{525750}$ & TTC32 & Tetratricopeptide repeat domain 32 & $51.6 \downarrow$ & 0.002 \\
\hline$\underline{504652}$ & TK1 & Thymidine kinase 1 , soluble & $51.5 \downarrow$ & 0.001 \\
\hline$\underline{\underline{506043}}$ & FKBP11 & FK506 binding protein $11,19 \mathrm{kda}$ & $51.1 \downarrow$ & 0.001 \\
\hline
\end{tabular}


Table 6.6. Supplemental (continued)

\begin{tabular}{|c|c|c|c|c|}
\hline Gene ID & Symbol & Full name & $\begin{array}{l}\text { Percent } \\
\text { change }^{1}\end{array}$ & P-Value \\
\hline$\underline{615149}$ & BLOC1S1 & Biogenesis of lysosomal organelles complex-1, subunit 1 & $50.8 \downarrow$ & 0.003 \\
\hline$\overline{100295432}$ & LOC100295432 & Uncharacterized LOC100295432 & $50.4 \downarrow$ & $<0.001$ \\
\hline 282485 & SULT1A1 & Sulfotransferase family, cytosolic, $1 \mathrm{~A}$, phenol-preferring, member 1 & $50.1 \downarrow$ & 0.003 \\
\hline$\overline{507166}$ & KRCC1 & Lysine-rich coiled-coil 1 & $49.9 \downarrow$ & $<0.001$ \\
\hline$\underline{505555}$ & VWA7 & Von Willebrand factor A domain containing 7 & $49.9 \downarrow$ & 0.001 \\
\hline$\underline{507276}$ & DHRS12 & Dehydrogenase/reductase (SDR family) member 12 & $49.9 \downarrow$ & 0.002 \\
\hline$\underline{516797}$ & PPT2 & Palmitoyl-protein thioesterase 2 & $49.2 \downarrow$ & $<0.001$ \\
\hline$\underline{515749}$ & CTNNAL1 & Catenin (cadherin-associated protein), alpha-like 1 & $49.1 \downarrow$ & 0.001 \\
\hline$\underline{514869}$ & TEX261 & Testis expressed 261 & $49.1 \downarrow$ & 0.001 \\
\hline$\underline{615610}$ & LOC615610 & Phosphoglycerate mutase 1-like & $48.9 \downarrow$ & 0.001 \\
\hline$\underline{506364}$ & SIGLEC8 & Sialic acid binding Ig-like lectin 8 & $48.8 \downarrow$ & 0.002 \\
\hline$\underline{785370}$ & LOC785370 & ATP-binding cassette, sub-family B (MDR/TAP), member 4-like & $48.6 \downarrow$ & 0.002 \\
\hline$\overline{511644}$ & TPMT & Thiopurine S-methyltransferase & $48.1 \downarrow$ & $<0.001$ \\
\hline$\underline{100295835}$ & LRRC70 & Leucine rich repeat containing 70 & $47.6 \downarrow$ & 0.002 \\
\hline$\underline{613491}$ & TMEM71 & Transmembrane protein 71 & $47.2 \downarrow$ & 0.002 \\
\hline$\underline{613597}$ & FBXO40 & F-box protein 40 & $47.1 \downarrow$ & $<0.001$ \\
\hline$\underline{100298453}$ & 100298453 & Myeloid-associated differentiation marker-like & $47.1 \downarrow$ & 0.001 \\
\hline$\underline{618441}$ & SEPX1 & Selenoprotein X, 1 & $46.8 \downarrow$ & 0.001 \\
\hline$\underline{404148}$ & PGAM1 & Phosphoglycerate mutase 1 (brain) & $46.5 \downarrow$ & 0.002 \\
\hline$\underline{281066}$ & CDK5 & Cyclin-dependent kinase 5 & $46.2 \downarrow$ & 0.001 \\
\hline$\underline{100298394}$ & LOC100298394 & Atpase, $\mathrm{H}+$ transporting, lysosomal $21 \mathrm{kda}, \mathrm{V} 0$ subunit b-like & $46.2 \downarrow$ & 0.003 \\
\hline$\underline{618612}$ & CRISP3 & Cysteine-rich secretory protein 3 & $46 \downarrow$ & $<0.001$ \\
\hline$\underline{508441}$ & CD33 & CD33 molecule & $45.7 \downarrow$ & 0.001 \\
\hline$\underline{505987}$ & GK & Glycerol kinase & $45.4 \downarrow$ & 0.002 \\
\hline$\underline{540113}$ & ATP6V1E2 & Atpase, $\mathrm{H}+$ transporting, lysosomal $31 \mathrm{kda}, \mathrm{V} 1$ subunit E2 & $45.3 \downarrow$ & 0.001 \\
\hline$\underline{616979}$ & RAB31 & RAB31, member RAS oncogene family & $45.3 \downarrow$ & 0.001 \\
\hline$\underline{510994}$ & ARF3 & ADP-ribosylation factor 3 & $45.1 \downarrow$ & $<0.001$ \\
\hline
\end{tabular}


Table 6.6. Supplemental (continued)

\begin{tabular}{|c|c|c|c|c|}
\hline Gene ID & Symbol & Full name & $\begin{array}{l}\text { Percent } \\
\text { change }^{1}\end{array}$ & P-Value \\
\hline$\underline{515291}$ & RMND5B & Required for meiotic nuclear division 5 homolog B (S. Cerevisiae) & $45 \downarrow$ & $<0.001$ \\
\hline$\overline{282860}$ & KIR2DL5A & Killer cell immunoglobulin-like receptor, two domains, long cytoplasmic tail, 5A & $44.6 \downarrow$ & $<0.001$ \\
\hline$\overline{539247}$ & APH1A & Anterior pharynx defective 1 homolog A (C. Elegans) & $43.7 \downarrow$ & $<0.001$ \\
\hline$\overline{785964}$ & LOC785964 & Uncharacterized LOC785964 & $43.4 \downarrow$ & 0.002 \\
\hline$\underline{616899}$ & C11H2orf68 & Chromosome 11 open reading frame, human c2orf68 & $43.3 \downarrow$ & $<0.001$ \\
\hline$\underline{616757}$ & BST1 & Bone marrow stromal cell antigen 1 & $43 \downarrow$ & 0.001 \\
\hline$\underline{407121}$ & NEB & Nebulin & $43 \downarrow$ & 0.002 \\
\hline$\underline{616003}$ & TM6SF1 & Transmembrane 6 superfamily member 1 & $42.6 \downarrow$ & $<0.001$ \\
\hline$\underline{618809}$ & VAT1L & Vesicle amine transport protein 1 homolog (T. Californica)-like & $42.6 \downarrow$ & $<0.001$ \\
\hline$\underline{100296268}$ & LST1 & Leukocyte specific transcript 1 & $42 \downarrow$ & 0.002 \\
\hline$\underline{493716}$ & IL18RAP & Interleukin 18 receptor accessory protein & $41.5 \downarrow$ & 0.001 \\
\hline$\underline{539596}$ & TGFBI & Transforming growth factor, beta-induced, $68 \mathrm{kda}$ & $41.3 \downarrow$ & $<0.001$ \\
\hline$\underline{613357}$ & ERP29 & Endoplasmic reticulum protein 29 & $41.2 \downarrow$ & 0.003 \\
\hline 282531 & GABARAPL2 & $\mathrm{GABA}(\mathrm{A})$ receptor-associated protein-like 2 & $40.8 \downarrow$ & 0.002 \\
\hline$\underline{514550}$ & ZCWPW1 & Zinc finger, CW type with PWWP domain 1 & $40.4 \downarrow$ & $<0.001$ \\
\hline$\underline{100298680}$ & PIGX & Phosphatidylinositol glycan anchor biosynthesis, class X & $40.4 \downarrow$ & 0.002 \\
\hline$\underline{444881}$ & MYD88 & Myeloid differentiation primary response gene (88) & $40.3 \downarrow$ & 0.003 \\
\hline$\underline{616169}$ & TCTA & T-cell leukemia translocation altered gene & $40.2 \downarrow$ & 0.001 \\
\hline$\underline{281139}$ & CELA1 & Chymotrypsin-like elastase family, member 1 & $39.8 \downarrow$ & 0.001 \\
\hline$\underline{338066}$ & CD97 & CD97 molecule & $39.8 \downarrow$ & 0.002 \\
\hline$\underline{515639}$ & RASSF8 & Ras association (ralgds/AF-6) domain family (N-terminal) member 8 & $39 \downarrow$ & 0.001 \\
\hline$\underline{518752}$ & ARG2 & Arginase, type II & $38.6 \downarrow$ & 0.002 \\
\hline$\underline{509439}$ & NECAP2 & NECAP endocytosis associated 2 & $38.5 \downarrow$ & 0.001 \\
\hline$\underline{282373}$ & SPADH1 & Spermadhesin 1 & $38.4 \downarrow$ & $<0.001$ \\
\hline$\underline{533526}$ & CCNE1 & Cyclin E1 & $37.6 \downarrow$ & 0.001 \\
\hline$\underline{507066}$ & SRSF7 & Serine/arginine-rich splicing factor 7 & $37.5 \downarrow$ & 0.001 \\
\hline$\underline{515548}$ & TREML2 & Triggering receptor expressed on myeloid cells-like 2 & $37 \downarrow$ & 0.001 \\
\hline
\end{tabular}


Table 6.6. Supplemental (continued)

\begin{tabular}{|c|c|c|c|c|}
\hline Gene ID & Symbol & Full name & $\begin{array}{l}\text { Percent } \\
\text { change }^{1}\end{array}$ & P-Value \\
\hline 768044 & NUDT2 & Nudix (nucleoside diphosphate linked moiety X)-type motif 2 & $36 \downarrow$ & 0.001 \\
\hline$\overline{505568}$ & EXOC7 & Exocyst complex component 7 & $35.9 \downarrow$ & 0.002 \\
\hline$\overline{788201}$ & LOC788201 & Uncharacterized LOC788201 & $35.7 \downarrow$ & $<0.001$ \\
\hline$\overline{538529}$ & SLC25A28 & Solute carrier family 25 , member 28 & $35.5 \downarrow$ & 0.002 \\
\hline 281976 & PDE6D & Phosphodiesterase 6D, cgmp-specific, rod, delta & $35.4 \downarrow$ & 0.002 \\
\hline$\underline{527124}$ & CDKN2B & Cyclin-dependent kinase inhibitor 2B (p15, inhibits CDK4) & $34.7 \downarrow$ & 0.001 \\
\hline$\underline{617785}$ & PILRA & Uncharacterized LOC617785 & $34 \downarrow$ & 0.002 \\
\hline$\underline{526702}$ & ACRC & Acidic repeat containing & $33.1 \downarrow$ & 0.002 \\
\hline$\underline{506622}$ & TMEM219 & Transmembrane protein 219 & $32.9 \downarrow$ & $<0.001$ \\
\hline$\underline{790027}$ & CCDC110 & Coiled-coil domain containing 110 & $32.9 \downarrow$ & 0.002 \\
\hline 282346 & SCARB1 & Scavenger receptor class B, member 1 & $32.6 \downarrow$ & 0.002 \\
\hline$\underline{617342}$ & CCDC83 & Coiled-coil domain containing 83 & $31.9 \downarrow$ & $<0.001$ \\
\hline$\underline{404547}$ & TREM1 & Triggering receptor expressed on myeloid cells 1 & $31.1 \downarrow$ & 0.001 \\
\hline$\underline{513275}$ & STX3 & Syntaxin 3 & $30.2 \downarrow$ & 0.001 \\
\hline$\underline{534293}$ & MCM3AP & Minichromosome maintenance complex component 3 associated protein & $30.1 \downarrow$ & 0.001 \\
\hline$\underline{616962}$ & C16H1orf105 & Chromosome 16 open reading frame, human c1orf105 & $29.8 \downarrow$ & 0.002 \\
\hline$\underline{538561}$ & MGEA5 & Meningioma expressed antigen 5 (hyaluronidase) & $29.1 \downarrow$ & 0.002 \\
\hline$\underline{526582}$ & TTPAL & Tocopherol (alpha) transfer protein-like & $28.8 \downarrow$ & $<0.001$ \\
\hline$\underline{521540}$ & ССТ6А & Chaperonin containing TCP1, subunit $6 \mathrm{~A}$ (zeta 1 ) & $28.5 \downarrow$ & 0.001 \\
\hline$\underline{533426}$ & INO80C & INO80 complex subunit $\mathrm{C}$ & $27.5 \downarrow$ & 0.001 \\
\hline$\underline{510042}$ & STARD3NL & STARD3 N-terminal like & $27.3 \downarrow$ & $<0.001$ \\
\hline$\underline{540667}$ & 8-Mar & Membrane-associated ring finger $(\mathrm{C} 3 \mathrm{HC} 4) 8$ & $27.2 \downarrow$ & 0.001 \\
\hline$\underline{751809}$ & WC1 & WC1 isolate DV9 & $26.5 \downarrow$ & 0.002 \\
\hline$\underline{533633}$ & WDR19 & WD repeat domain 19 & $26.1 \downarrow$ & 0.001 \\
\hline 282183 & AP2B1 & Adaptor-related protein complex 2 , beta 1 subunit & $25.9 \downarrow$ & 0.001 \\
\hline$\underline{100337350}$ & 100337350 & Glycogen synthase kinase 3 beta-like & $25.5 \downarrow$ & 0.001 \\
\hline$\underline{768316}$ & NRBF2 & Nuclear receptor binding factor 2 & $24.8 \downarrow$ & 0.003 \\
\hline
\end{tabular}


Table 6.6. Supplemental (continued)

\begin{tabular}{|c|c|c|c|c|}
\hline Gene ID & Symbol & Full name & $\begin{array}{l}\text { Percent } \\
\text { change }^{1}\end{array}$ & P-Value \\
\hline$\underline{506612}$ & DTNBP1 & Dystrobrevin binding protein 1 & $24.3 \downarrow$ & 0.003 \\
\hline$\underline{528593}$ & CD5L & CD5 molecule-like & $23.3 \downarrow$ & 0.001 \\
\hline$\overline{528668}$ & KLHL5 & Kelch-like 5 (Drosophila) & $22 \downarrow$ & 0.001 \\
\hline$\overline{528654}$ & VPS41 & Vacuolar protein sorting 41 homolog (S. Cerevisiae) & $21.7 \downarrow$ & 0.001 \\
\hline
\end{tabular}

LPS-activated PMNL (50 $\mu \mathrm{g} / \mathrm{ml}$ E. Coli LPS)

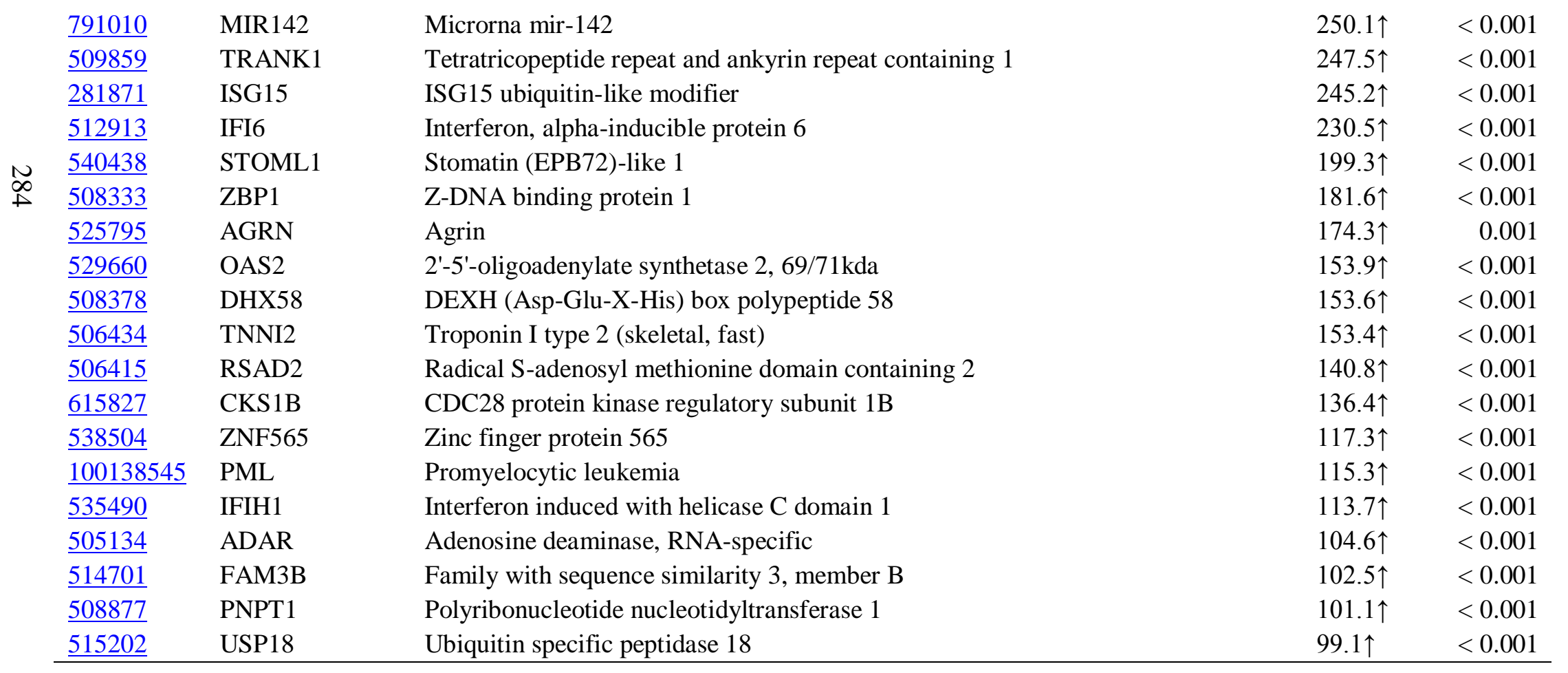


Table 6.6. Supplemental (continued)

\begin{tabular}{|c|c|c|c|c|}
\hline Gene ID & Symbol & Full name & $\begin{array}{l}\text { Percent } \\
\text { change }^{1}\end{array}$ & P-Value \\
\hline 347700 & EIF2AK2 & Eukaryotic translation initiation factor 2 -alpha kinase 2 & $96.1 \uparrow$ & $<0.001$ \\
\hline$\overline{509283}$ & RNF213 & Ring finger protein 213 & $95.4 \uparrow$ & $<0.001$ \\
\hline$\underline{513185}$ & PARP12 & Poly (ADP-ribose) polymerase family, member 12 & $89.9 \uparrow$ & $<0.001$ \\
\hline$\overline{504760}$ & DDX58 & DEAD (Asp-Glu-Ala-Asp) box polypeptide 58 & $87.6 \uparrow$ & $<0.001$ \\
\hline$\underline{509620}$ & FTSJD2 & Ftsj methyltransferase domain containing 2 & $87.3 \uparrow$ & $<0.001$ \\
\hline$\underline{516599}$ & LOC516599 & Tripartite motif containing 5-like & $85 \uparrow$ & $<0.001$ \\
\hline$\underline{514492}$ & TRIM6 & Tripartite motif containing 6 & $75.1 \uparrow$ & $<0.001$ \\
\hline$\underline{613533}$ & FLNB & Filamin B, beta & $68.7 \uparrow$ & $<0.001$ \\
\hline$\overline{782527}$ & LOC782527 & Tripartite motif protein 5 alpha-like & $56.5 \uparrow$ & $<0.001$ \\
\hline$\overline{513479}$ & RNF114 & Ring finger protein 114 & $47.2 \uparrow$ & $<0.001$ \\
\hline$\underline{100300995}$ & LOC100300995 & Cytochrome c oxidase subunit III-like & $87.7 \downarrow$ & $<0.001$ \\
\hline$\underline{281889}$ & KRT17 & Keratin 17 & $79.3 \downarrow$ & $<0.001$ \\
\hline$\overline{614288}$ & MKKS & Mckusick-Kaufman syndrome & $76.4 \downarrow$ & $<0.001$ \\
\hline$\underline{505407}$ & IGHE & Immunoglobulin heavy constant epsilon & $75.3 \downarrow$ & $<0.001$ \\
\hline$\underline{505653}$ & MS4A1 & Membrane-spanning 4-domains, subfamily A, member 1 & $73.9 \downarrow$ & $<0.001$ \\
\hline$\overline{100300424}$ & CAPNS2 & Calpain, small subunit 2 & $62.4 \downarrow$ & $<0.001$ \\
\hline$\underline{505719}$ & FAM63A & Family with sequence similarity 63 , member A & $59.6 \downarrow$ & $<0.001$ \\
\hline$\overline{514978}$ & LOC514978 & Lipopolysaccharide-binding protein-like & $58.1 \downarrow$ & $<0.001$ \\
\hline$\overline{100139105}$ & RAB3D & RAB3D, member RAS oncogene family & $57.4 \downarrow$ & $<0.001$ \\
\hline$\overline{100295432}$ & LOC100295432 & Uncharacterized LOC100295432 & $55.5 \downarrow$ & $<0.001$ \\
\hline$\underline{535768}$ & MTO1 & Mitochondrial translation optimization 1 homolog (S. Cerevisiae) & $52.9 \downarrow$ & $<0.001$ \\
\hline$\overline{507166}$ & $\mathrm{KRCC} 1$ & Lysine-rich coiled-coil 1 & $48.5 \downarrow$ & $<0.001$ \\
\hline$\underline{618612}$ & CRISP3 & Cysteine-rich secretory protein 3 & $45.2 \downarrow$ & $<0.001$ \\
\hline$\underline{511644}$ & TPMT & Thiopurine S-methyltransferase & $43.7 \downarrow$ & $<0.001$ \\
\hline$\overline{618809}$ & VAT1L & Vesicle amine transport protein 1 homolog (T. Californica)-like & $40.9 \downarrow$ & $<0.001$ \\
\hline$\overline{788201}$ & LOC788201 & Uncharacterized LOC788201 & $32.1 \downarrow$ & $<0.001$ \\
\hline 282183 & AP2B1 & Adaptor-related protein complex 2 , beta 1 subunit & $28.3 \downarrow$ & $<0.001$ \\
\hline
\end{tabular}


Table 6.6. Supplemental (continued)

\begin{tabular}{|c|c|c|c|c|}
\hline Gene ID & Symbol & Full name & $\begin{array}{l}\text { Percent } \\
\text { change }^{1}\end{array}$ & P-Value \\
\hline$\underline{517126}$ & LOC517126 & Fc receptor-like protein 5-like & $25.1 \downarrow$ & $<0.001$ \\
\hline
\end{tabular}

${ }^{\mathrm{T}}$ Direction of change in expression of genes is indicated with a $\uparrow$ or $\downarrow$.

${ }^{2}$ Neutrophils were collected on day $6.4 \pm 1$ postpartum from cows fed a basal diet with no added dietary trace minerals $($ basal, $\mathrm{n}=$ 7) or supplemented with $200 \mathrm{mg} \mathrm{Mn,} 25 \mathrm{mg} \mathrm{Co}, 125 \mathrm{mg} \mathrm{Cu}$, and $360 \mathrm{mg} \mathrm{Zn}$ from sulfate and carbonate sources (inorganic, $\mathrm{n}=7$ ) or supplied by amino acid complexes of $\mathrm{Mn}, \mathrm{Cu}$, and $\mathrm{Zn}$, and cobalt glucoheptonate (organic, $\mathrm{n}=7$ ) after incubation with 0 or 50 $\mu \mathrm{g} / \mathrm{mL}$ of $E$. coli lipopolysaccharide for $120 \mathrm{~min}$ 
Table 6.7. Supplemental. Top 100 genes with differential expression in neutrophils (PMNL) collected from cows either on day $58.1 \pm 1.0$ prepartum or $6.4 \pm 0.1$ postpartum after incubation with 0 or $50 \mu \mathrm{g} / \mathrm{mL}$ of $E$. coli lipopolysaccharide (LPS)

\begin{tabular}{|c|c|c|c|c|}
\hline Gene ID & Symbol & Full name & $\begin{array}{l}\text { Percent } \\
\text { change }^{1}\end{array}$ & P-Value \\
\hline \multicolumn{5}{|c|}{ Postpartum vs prepartum in non-activated PMNL $(0 \mu \mathrm{g} / \mathrm{ml} E . \text { Coli LPS })^{2}$} \\
\hline \multicolumn{5}{|c|}{ Down-regulated genes } \\
\hline$\underline{510907}$ & ADAM19 & ADAM metallopeptidase domain 19 & $100 \downarrow$ & $<0.001$ \\
\hline 280729 & $\mathrm{~B} 2 \mathrm{M}$ & Beta-2-microglobulin & $100 \downarrow$ & $<0.001$ \\
\hline 286842 & BASP1 & Brain abundant, membrane attached signal protein 1 & $100 \downarrow$ & $<0.001$ \\
\hline$\underline{10012604}$ & BOLA-N & MHC class I antigen & $100 \downarrow$ & $<0.001$ \\
\hline 281032 & BTG1 & B-cell translocation gene 1, anti-proliferative & $100 \downarrow$ & $<0.001$ \\
\hline$\underline{512514}$ & $\mathrm{CD} 27$ & $\mathrm{CD} 27$ molecule & $100 \downarrow$ & $<0.001$ \\
\hline$\underline{281705}$ & CNTN1 & Contactin 1 & $100 \downarrow$ & $<0.001$ \\
\hline$\underline{527934}$ & CORO7 & Coronin 7 & $100 \downarrow$ & $<0.001$ \\
\hline$\overline{508482}$ & CPNE5 & Copine V & $100 \downarrow$ & $<0.001$ \\
\hline$\underline{539235}$ & CSNK2B & Casein kinase 2 , beta polypeptide & $100 \downarrow$ & $<0.001$ \\
\hline$\underline{538021}$ & DAGLB & Diacylglycerol lipase, beta & $100 \downarrow$ & $<0.001$ \\
\hline$\underline{539175}$ & DUSP1 & Dual specificity phosphatase 1 & $100 \downarrow$ & $<0.001$ \\
\hline$\underline{539140}$ & DUSP2 & Dual specificity phosphatase 2 & $100 \downarrow$ & $<0.001$ \\
\hline$\underline{514259}$ & EFHD2 & EF-hand domain family, member D2 & $100 \downarrow$ & $<0.001$ \\
\hline$\underline{526347}$ & EMR3 & EGF-like module-containing mucin-like hormone receptor-like 3-like & $100 \downarrow$ & $<0.001$ \\
\hline$\underline{281251}$ & IL1B & Interleukin 1 , beta & $100 \downarrow$ & $<0.001$ \\
\hline$\underline{281860}$ & IL1RN & Interleukin 1 receptor antagonist & $100 \downarrow$ & $<0.001$ \\
\hline$\underline{280828}$ & IL8 & Interleukin 8 & $100 \downarrow$ & $<0.001$ \\
\hline$\underline{337917}$ & IRF1 & Interferon regulatory factor 1 & $100 \downarrow$ & $<0.001$ \\
\hline$\underline{281256}$ & ITM2C & Integral membrane protein $2 \mathrm{C}$ & $100 \downarrow$ & $<0.001$ \\
\hline$\underline{407173}$ & JSP.1 & Mhe class i jsp. 1 & $100 \downarrow$ & $<0.001$ \\
\hline
\end{tabular}


Table 6.7. Supplemental (continued)

\begin{tabular}{|c|c|c|c|c|}
\hline Gene ID & Symbol & Full name & $\begin{array}{l}\text { Percent } \\
\text { change }^{1}\end{array}$ & P-Value \\
\hline 514246 & JUNB & Jun B proto-oncogene & $100 \downarrow$ & $<0.001$ \\
\hline$\underline{10012591}$ & LOC10012591 & Uncharacterized protein 100125016 & $100 \downarrow$ & $<0.001$ \\
\hline$\underline{10013849}$ & LOC10013849 & Uncharacterized LOC100138493 & $100 \downarrow$ & $<0.001$ \\
\hline$\underline{10029659}$ & LOC10029659 & Uncharacterized LOC100296593 & $100 \downarrow$ & $<0.001$ \\
\hline 281370 & UBB & Ubiquitin B & $100 \downarrow$ & $<0.001$ \\
\hline$\underline{508666}$ & LOC508666 & C-C motif chemokine 23 & $100 \downarrow$ & $<0.001$ \\
\hline$\underline{509972}$ & LOC509972 & C-type lectin domain family 2 member D11-like & $100 \downarrow$ & $<0.001$ \\
\hline$\underline{529195}$ & MEFV & Mediterranean fever & $100 \downarrow$ & $<0.001$ \\
\hline$\underline{517231}$ & KIAA0226L & KIAA0226-like ortholog & $100 \downarrow$ & $<0.001$ \\
\hline$\underline{514626}$ & MXD1 & MAX dimerization protein 1 & $100 \downarrow$ & $<0.001$ \\
\hline$\underline{539780}$ & NAIP & NLR family, apoptosis inhibitory protein & $100 \downarrow$ & $<0.001$ \\
\hline$\underline{281345}$ & NCF1 & Neutrophil cytosolic factor 1 & $100 \downarrow$ & $<0.001$ \\
\hline$\underline{282291}$ & NFKBIA & Nuclear factor of kappa light polypeptide gene enhancer in B-cells inhibitor, alpha & $100 \downarrow$ & $<0.001$ \\
\hline$\underline{513730}$ & NOTCH2 & Notch 2 & $100 \downarrow$ & $<0.001$ \\
\hline$\underline{10012450}$ & PDE4B & Phosphodiesterase 4B, camp-specific & $100 \downarrow$ & $<0.001$ \\
\hline$\underline{513055}$ & RGS2 & Regulator of G-protein signaling 2, 24kda & $100 \downarrow$ & $<0.001$ \\
\hline$\underline{534171}$ & SAMSN1 & SAM domain, SH3 domain and nuclear localization signals 1 & $100 \downarrow$ & $<0.001$ \\
\hline$\underline{508861}$ & SAT1 & Spermidine/spermine N1-acetyltransferase 1 & $100 \downarrow$ & $<0.001$ \\
\hline$\underline{514346}$ & SDS & Serine dehydratase & $100 \downarrow$ & $<0.001$ \\
\hline$\underline{510239}$ & SEMA4A & Sema domain, immunoglobulin domain (Ig), transmembrane domain (TM) and & $100 \downarrow$ & $<0.001$ \\
\hline$\underline{281375}$ & SERPINE1 & Serpin peptidase inhibitor, clade E (nexin, plasminogen activator inhibitor type 1), & $100 \downarrow$ & $<0.001$ \\
\hline$\underline{327666}$ & SIRPA & Signal-regulatory protein alpha & $100 \downarrow$ & $<0.001$ \\
\hline$\underline{533166}$ & SORL1 & Sortilin-related receptor, L(DLR class) A repeats containing & $100 \downarrow$ & $<0.001$ \\
\hline$\underline{509501}$ & SRGN & Serglycin & $100 \downarrow$ & $<0.001$ \\
\hline$\underline{512652}$ & $\mathrm{SSH} 2$ & Slingshot homolog 2 (Drosophila) & $100 \downarrow$ & $<0.001$ \\
\hline$\underline{505080}$ & TGM3 & Transglutaminase 3 (E polypeptide, protein-glutamine-gamma-glutamyltransferase) & $100 \downarrow$ & $<0.001$ \\
\hline
\end{tabular}


Table 6.7. Supplemental (continued)

\begin{tabular}{|c|c|c|c|c|}
\hline Gene ID & Symbol & Full name & $\begin{array}{l}\text { Percent } \\
\text { change }^{1}\end{array}$ & P-Value \\
\hline$\underline{508105}$ & TNFAIP3 & Tumor necrosis factor, alpha-induced protein 3 & $100 \downarrow$ & $<0.001$ \\
\hline$\underline{526103}$ & TNIP1 & TNFAIP3 interacting protein 1 & $100 \downarrow$ & $<0.001$ \\
\hline$\underline{506790}$ & TXNIP & Thioredoxin interacting protein & $100 \downarrow$ & $<0.001$ \\
\hline 444874 & UBC & Ubiquitin $\mathrm{C}$ & $100 \downarrow$ & $<0.001$ \\
\hline$\underline{534929}$ & VNN2 & Vanin 2 & $100 \downarrow$ & $<0.001$ \\
\hline 280960 & $\mathrm{XDH}$ & Xanthine dehydrogenase & $100 \downarrow$ & $<0.001$ \\
\hline 282127 & ZFP36 & Zinc finger protein $36, \mathrm{C} 3 \mathrm{H}$ type, homolog (mouse) & $100 \downarrow$ & $<0.001$ \\
\hline$\underline{507417}$ & ABTB1 & Ankyrin repeat and BTB (POZ) domain containing 1 & $99.9 \downarrow$ & $<0.001$ \\
\hline$\underline{536628}$ & ACSL4 & Acyl-coa synthetase long-chain family member 4 & $99.9 \downarrow$ & $<0.001$ \\
\hline$\underline{280979}$ & ACTB & Actin, beta & $99.9 \downarrow$ & $<0.001$ \\
\hline$\underline{504288}$ & AKAP13 & A kinase (PRKA) anchor protein 13 & $99.9 \downarrow$ & $<0.001$ \\
\hline$\underline{509575}$ & AMICA1 & Adhesion molecule, interacts with CXADR antigen 1 & $99.9 \downarrow$ & $<0.001$ \\
\hline$\underline{519803}$ & AMPD3 & Adenosine monophosphate deaminase 3 & $99.9 \downarrow$ & $<0.001$ \\
\hline$\underline{532856}$ & ANKRD11 & Ankyrin repeat domain 11 & $99.9 \downarrow$ & $<0.001$ \\
\hline$\underline{510080}$ & ANTXR2 & Anthrax toxin receptor 2 & $99.9 \downarrow$ & $<0.001$ \\
\hline$\underline{511889}$ & ARAP1 & Arfgap with rhogap domain, ankyrin repeat and PH domain 1 & $99.9 \downarrow$ & $<0.001$ \\
\hline$\underline{538835}$ & ARHGAP30 & Rho gtpase activating protein 30 & $99.9 \downarrow$ & $<0.001$ \\
\hline$\underline{538517}$ & ARRDC4 & Arrestin domain containing 4 & $99.9 \downarrow$ & $<0.001$ \\
\hline$\underline{519758}$ & ATP1B1 & Atpase, $\mathrm{Na}+\mathrm{K}+$ transporting, beta 1 polypeptide & $99.9 \downarrow$ & $<0.001$ \\
\hline$\underline{508723}$ & ATP8A2 & Atpase, aminophospholipid transporter, class I, type 8A, member 2 & $99.9 \downarrow$ & $<0.001$ \\
\hline$\underline{531942}$ & BCL3 & B-cell CLL/lymphoma 3 & $99.9 \downarrow$ & $<0.001$ \\
\hline$\overline{514386}$ & BIRC3 & Baculoviral IAP repeat containing 3 & $99.9 \downarrow$ & $<0.001$ \\
\hline$\underline{505676}$ & BOLA & MHC class I heavy chain & $99.9 \downarrow$ & $<0.001$ \\
\hline$\underline{510417}$ & BOLA-NC1 & Non-classical MHC class I antigen & $99.9 \downarrow$ & $<0.001$ \\
\hline$\underline{510417}$ & BOLA-NC1 & Non-classical MHC class I antigen & $99.9 \downarrow$ & $<0.001$ \\
\hline 539364 & BTG2 & BTG family, member 2 & $99.9 \downarrow$ & $<0.001$ \\
\hline
\end{tabular}


Table 6.7. Supplemental (continued)

\begin{tabular}{|c|c|c|c|c|}
\hline Gene ID & Symbol & Full name & $\begin{array}{l}\text { Percent } \\
\text { change }^{1}\end{array}$ & P-Value \\
\hline 512628 & C21H14orf79 & Chromosome 21 open reading frame, human c14orf79 & $99.9 \downarrow$ & $<0.001$ \\
\hline$\overline{532940}$ & CCDC28A & Coiled-coil domain containing 28A & $99.9 \downarrow$ & $<0.001$ \\
\hline 511112 & CCL21 & Chemokine (C-C motif) ligand 21 & $99.9 \downarrow$ & $<0.001$ \\
\hline 281668 & $\mathrm{CCNB} 2$ & Cyclin B2 & $99.9 \downarrow$ & $<0.001$ \\
\hline$\underline{509717}$ & $\mathrm{CCNC}$ & Cyclin C & $99.9 \downarrow$ & $<0.001$ \\
\hline$\underline{505200}$ & $\mathrm{CCNF}$ & Cyclin F & $99.9 \downarrow$ & $<0.001$ \\
\hline$\underline{506713}$ & CD82 & CD82 molecule & $99.9 \downarrow$ & $<0.001$ \\
\hline$\underline{520428}$ & CDC14B & CDC14 cell division cycle 14 homolog B (S. Cerevisiae) & $99.9 \downarrow$ & $<0.001$ \\
\hline$\overline{508916}$ & CES1 & Carboxylesterase 1 (monocyte/macrophage serine esterase 1) & $99.9 \downarrow$ & $<0.001$ \\
\hline 526697 & CNTN3 & Contactin 3 (plasmacytoma associated) & $99.9 \downarrow$ & $<0.001$ \\
\hline$\overline{614728}$ & COASY & Coa synthase & $99.9 \downarrow$ & $<0.001$ \\
\hline$\overline{540286}$ & DAZAP2 & DAZ associated protein 2 & $99.9 \downarrow$ & $<0.001$ \\
\hline$\overline{529235}$ & DDIT4 & DNA-damage-inducible transcript 4 & $99.9 \downarrow$ & $<0.001$ \\
\hline$\underline{614215}$ & DDX39A & DEAD (Asp-Glu-Ala-Asp) box polypeptide 39A & $99.9 \downarrow$ & $<0.001$ \\
\hline$\underline{510093}$ & DDX3X & DEAD (Asp-Glu-Ala-Asp) box polypeptide 3, X-linked & $99.9 \downarrow$ & $<0.001$ \\
\hline$\underline{533700}$ & DDX5 & DEAD (Asp-Glu-Ala-Asp) box polypeptide 5 & $99.9 \downarrow$ & $<0.001$ \\
\hline$\underline{513906}$ & DDX6 & DEAD (Asp-Glu-Ala-Asp) box polypeptide 6 & $99.9 \downarrow$ & $<0.001$ \\
\hline$\underline{534251}$ & DEDD2 & Death effector domain containing 2 & $99.9 \downarrow$ & $<0.001$ \\
\hline 784484 & DENND5A & DENN/MADD domain containing 5A & $99.9 \downarrow$ & $<0.001$ \\
\hline 404129 & DGAT2 & Diacylglycerol O-acyltransferase 2 & $99.9 \downarrow$ & $<0.001$ \\
\hline$\underline{537533}$ & DGKH & Diacylglycerol kinase, eta & $99.9 \downarrow$ & $<0.001$ \\
\hline$\overline{511691}$ & DNM2 & Dynamin 2 & $99.9 \downarrow$ & $<0.001$ \\
\hline 281133 & ECE1 & Endothelin converting enzyme 1 & $99.9 \downarrow$ & $<0.001$ \\
\hline 788760 & EHBP1L1 & EH domain binding protein 1-like 1 & $99.9 \downarrow$ & $<0.001$ \\
\hline$\underline{511908}$ & EHD1 & EH-domain containing 1 & $99.9 \downarrow$ & $<0.001$ \\
\hline$\underline{509764}$ & EIF1 & Eukaryotic translation initiation factor 1 & $99.9 \downarrow$ & $<0.001$ \\
\hline
\end{tabular}


Table 6.7. Supplemental (continued)

\begin{tabular}{|c|c|c|c|c|}
\hline Gene ID & Symbol & Full name & $\begin{array}{l}\text { Percent } \\
\text { change }^{1}\end{array}$ & P-Value \\
\hline 286870 & EIF4G2 & Eukaryotic translation initiation factor 4 gamma, 2 & $99.9 \downarrow$ & $<0.001$ \\
\hline
\end{tabular}

Postpartum vs. Prepartum in LPS-activated PMNL $(50 \mu \mathrm{g} / \mathrm{ml} \text { E. Coli LPS })^{2}$

Up-regulated

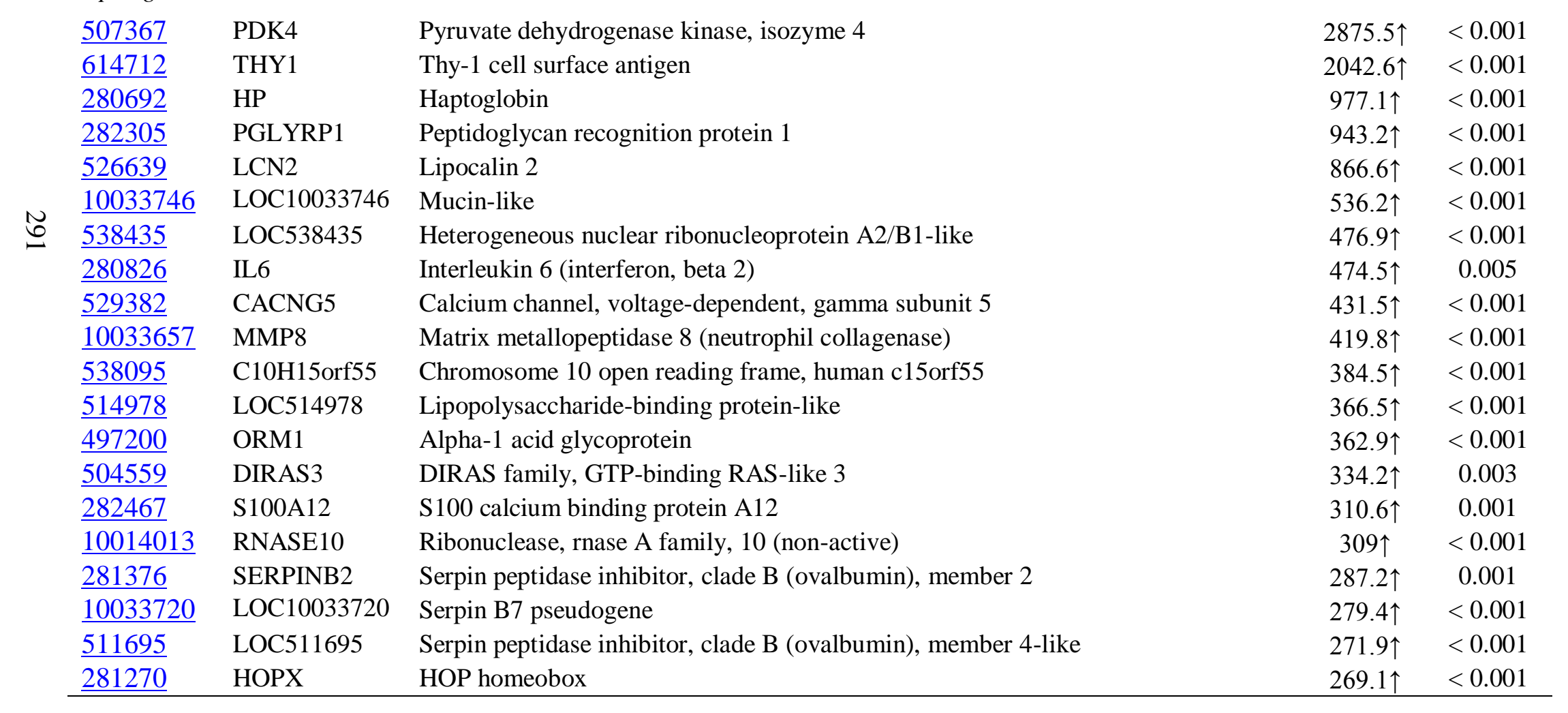


Table 6.7. Supplemental (continued)

\begin{tabular}{|c|c|c|c|c|}
\hline Gene ID & Symbol & Full name & $\begin{array}{l}\text { Percent } \\
\text { change }^{1}\end{array}$ & P-Value \\
\hline$\underline{521616}$ & CACNG6 & Calcium channel, voltage-dependent, gamma subunit 6 & $264.3 \uparrow$ & 0.001 \\
\hline$\overline{527889}$ & CSNK1G1 & Casein kinase 1 , gamma 1 & $261 \uparrow$ & $<0.001$ \\
\hline$\underline{532569}$ & S100A9 & S100 calcium binding protein A9 & $260.9 \uparrow$ & $<0.001$ \\
\hline 618664 & ART5 & ADP-ribosyltransferase 5 & $249.7 \uparrow$ & $<0.001$ \\
\hline$\underline{511106}$ & LOC511106 & Serpin peptidase inhibitor, clade B like & $247.1 \uparrow$ & $<0.001$ \\
\hline 517857 & $\mathrm{C} 3 \mathrm{H} 1$ orf 123 & Chromosome 3 open reading frame, human c1orf123 & $245 \uparrow$ & $<0.001$ \\
\hline$\underline{10033724}$ & LOC10033724 & Serpin peptidase inhibitor, clade B (ovalbumin), member 3-like & $242.7 \uparrow$ & $<0.001$ \\
\hline$\underline{10033715}$ & LOC10033715 & Serpin peptidase inhibitor, clade B like-like & $241.3 \uparrow$ & $<0.001$ \\
\hline$\underline{618956}$ & SERPINB4 & Serpin peptidase inhibitor, clade B (ovalbumin), member 4 & $241.1 \uparrow$ & $<0.001$ \\
\hline 280726 & ASS1 & Argininosuccinate synthase 1 & $240.7 \uparrow$ & $<0.001$ \\
\hline$\underline{10033605}$ & LOC10033605 & Uncharacterized LOC100336055 & $240.6 \uparrow$ & $<0.001$ \\
\hline$\underline{787178}$ & LOC787178 & SCAN domain containing protein 1-like pseudogene & $240.4 \uparrow$ & $<0.001$ \\
\hline$\overline{281754}$ & EPB42 & Erythrocyte membrane protein band 4.2 & $238.1 \uparrow$ & 0.001 \\
\hline$\underline{515082}$ & OLFM4 & Olfactomedin 4 & $237.1 \uparrow$ & $<0.001$ \\
\hline$\underline{10033722}$ & LOC10033722 & Serpin peptidase inhibitor, clade B (ovalbumin), member 4-like & $225.8 \uparrow$ & $<0.001$ \\
\hline 790312 & LOC790312 & Purine nucleoside phosphorylase-like & $224.9 \uparrow$ & $<0.001$ \\
\hline$\underline{493724}$ & PNP & Purine nucleoside phosphorylase & $224.1 \uparrow$ & $<0.001$ \\
\hline$\underline{786410}$ & LOC786410 & Serpin peptidase inhibitor, clade B like & $224 \uparrow$ & $<0.001$ \\
\hline$\underline{10030042}$ & CAPNS2 & Calpain, small subunit 2 & $223.8 \uparrow$ & $<0.001$ \\
\hline$\underline{493724}$ & PNP & Purine nucleoside phosphorylase & $221.5 \uparrow$ & $<0.001$ \\
\hline$\underline{616818}$ & S100A8 & S100 calcium binding protein $\mathrm{A} 8$ & $219.6 \uparrow$ & 0.001 \\
\hline$\underline{10033521}$ & LOC10033521 & Heme-binding protein 1 pseudogene & $217.5 \uparrow$ & $<0.001$ \\
\hline$\underline{538461}$ & KBTBD6 & Kelch repeat and BTB (POZ) domain containing 6 & $212.7 \uparrow$ & $<0.001$ \\
\hline 282123 & VLDLR & Very low density lipoprotein receptor & $209.4 \uparrow$ & $<0.001$ \\
\hline$\underline{10029494}$ & LOC10029494 & S100 calcium binding protein A11-like & $204.5 \uparrow$ & 0.005 \\
\hline 782900 & C22H3orf78 & Chromosome 22 open reading frame, human c3orf78 & $199.9 \uparrow$ & $<0.001$ \\
\hline
\end{tabular}


Table 6.7. Supplemental (continued)

\begin{tabular}{|c|c|c|c|c|}
\hline Gene ID & Symbol & Full name & $\begin{array}{l}\text { Percent } \\
\text { change }^{1}\end{array}$ & P-Value \\
\hline 506060 & CSNK1G2 & Casein kinase 1 , gamma 2 & $199.8 \uparrow$ & $<0.001$ \\
\hline$\overline{613416}$ & LOC613416 & Heme-binding protein 1 pseudogene & $199.1 \uparrow$ & $<0.001$ \\
\hline$\underline{280686}$ & $\mathrm{~F} 3$ & Coagulation factor III (thromboplastin, tissue factor) & $196.5 \uparrow$ & 0.006 \\
\hline$\overline{520243}$ & SLC38A6 & Solute carrier family 38, member 6 & $192.2 \uparrow$ & 0.001 \\
\hline$\overline{504593}$ & SPRY4 & Sprouty homolog 4 (Drosophila) & $190.2 \uparrow$ & $<0.001$ \\
\hline$\underline{618550}$ & LOC618550 & Heme-binding protein 1 pseudogene & $189 \uparrow$ & $<0.001$ \\
\hline 282885 & PRDX5 & Peroxiredoxin 5 & $186.2 \uparrow$ & $<0.001$ \\
\hline$\underline{509600}$ & GPNMB & Glycoprotein (transmembrane) nmb & $184.1 \uparrow$ & $<0.001$ \\
\hline$\underline{538751}$ & AREGB & Amphiregulin B & $183.8 \uparrow$ & $<0.001$ \\
\hline$\underline{518758}$ & SLC25A20 & Solute carrier family 25 (carnitine/acylcarnitine translocase), member 20 & $182.3 \uparrow$ & $<0.001$ \\
\hline$\underline{528055}$ & ANXA9 & Annexin A9 & $179.5 \uparrow$ & $<0.001$ \\
\hline$\underline{281049}$ & $\mathrm{CD} 24$ & CD24 antigen & $178.9 \uparrow$ & $<0.001$ \\
\hline$\underline{10012523}$ & CCDC96 & Coiled-coil domain containing 96 & $178.3 \uparrow$ & $<0.001$ \\
\hline$\underline{10029746}$ & LOC10029746 & Cd24 molecule-like & $178.2 \uparrow$ & 0.001 \\
\hline 783627 & FAM162B & Family with sequence similarity 162 , member B & $176.5 \uparrow$ & 0.002 \\
\hline$\underline{532668}$ & ZNF821 & Zinc finger protein 821 & $175.6 \uparrow$ & $<0.001$ \\
\hline$\underline{535327}$ & MAPK13 & Mitogen-activated protein kinase 13 & $174.3 \uparrow$ & $<0.001$ \\
\hline$\underline{510097}$ & FOXRED1 & FAD-dependent oxidoreductase domain containing 1 & $170.2 \uparrow$ & $<0.001$ \\
\hline 516067 & FOLR1 & Folate receptor 1 (adult) & $169.5 \uparrow$ & $<0.001$ \\
\hline$\underline{505318}$ & OLFML3 & Olfactomedin-like 3 & $169 \uparrow$ & $<0.001$ \\
\hline$\underline{512583}$ & CCBP2 & Chemokine binding protein 2 & $167.1 \uparrow$ & $<0.001$ \\
\hline$\overline{508158}$ & AHCY & Adenosylhomocysteinase & $166.7 \uparrow$ & $<0.001$ \\
\hline$\underline{613880}$ & LOC613880 & Ribonuclease UK114 pseudogene & $166.4 \uparrow$ & $<0.001$ \\
\hline 280986 & AGER & Advanced glycosylation end product-specific receptor & $165.8 \uparrow$ & 0.001 \\
\hline 782956 & $\mathrm{CCDC} 126$ & Coiled-coil domain containing 126 & $163.6 \uparrow$ & $<0.001$ \\
\hline$\underline{781275}$ & SAPCD1 & Suppressor APC domain containing 1 & $161.2 \uparrow$ & $<0.001$ \\
\hline
\end{tabular}


Table 6.7. Supplemental (continued)

\begin{tabular}{|c|c|c|c|c|}
\hline Gene ID & Symbol & Full name & $\begin{array}{l}\text { Percent } \\
\text { change }^{1}\end{array}$ & P-Value \\
\hline 512748 & AMPD1 & Adenosine monophosphate deaminase 1 & $160.4 \uparrow$ & 0.001 \\
\hline$\overline{767920}$ & MGC127538 & Uncharacterized protein MGC127538 & $160.1 \uparrow$ & 0.001 \\
\hline$\underline{531514}$ & $\mathrm{BMX}$ & BMX non-receptor tyrosine kinase & $157.8 \uparrow$ & $<0.001$ \\
\hline 10029547 & EREG & Epiregulin & $157 \uparrow$ & 0.003 \\
\hline 517251 & TMEM242 & Transmembrane protein 242 & $156.3 \uparrow$ & 0.001 \\
\hline 10029494 & C7H19orf38 & Chromosome 7 open reading frame, human c19orf38 & $156 \uparrow$ & $<0.001$ \\
\hline$\underline{506110}$ & $\mathrm{C} 16 \mathrm{H} 1$ orf 187 & Chromosome 16 open reading frame, human c1orf187 & $156 \uparrow$ & 0.004 \\
\hline$\underline{505719}$ & FAM63A & Family with sequence similarity 63 , member A & $154.8 \uparrow$ & $<0.001$ \\
\hline$\underline{617906}$ & POP7 & Processing of precursor 7, ribonuclease P/MRP subunit (S. Cerevisiae) & $152.4 \uparrow$ & $<0.001$ \\
\hline 508167 & RRM2 & Ribonucleotide reductase M2 & $151.2 \uparrow$ & $<0.001$ \\
\hline$\underline{616375}$ & LAMTOR2 & Late endosomal/lysosomal adaptor, MAPK and MTOR activator 2 & $149.2 \uparrow$ & $<0.001$ \\
\hline$\underline{282871}$ & MMP9 & Matrix metallopeptidase 9 (gelatinase B, 92kda gelatinase, 92kda type IV & $146.8 \uparrow$ & $<0.001$ \\
\hline$\overline{504741}$ & COLEC12 & Collectin sub-family member 12 & $146.2 \uparrow$ & $<0.001$ \\
\hline 540643 & LMNB1 & Lamin B1 & $146 \uparrow$ & $<0.001$ \\
\hline$\underline{618503}$ & UQCC & Ubiquinol-cytochrome c reductase complex chaperone & $145.6 \uparrow$ & $<0.001$ \\
\hline 280828 & IL8 & Interleukin 8 & $142.8 \uparrow$ & $<0.001$ \\
\hline$\underline{512571}$ & PKM2 & Pyruvate kinase, muscle & $140.9 \uparrow$ & $<0.001$ \\
\hline$\underline{784359}$ & LOC784359 & UDP-glcnac:betagal beta-1,3-N-acetylglucosaminyltransferase 2-like & $140.2 \uparrow$ & $<0.001$ \\
\hline$\underline{528221}$ & TRMT5 & TRM5 trna methyltransferase 5 homolog (S. Cerevisiae) & $138.9 \uparrow$ & 0.006 \\
\hline$\underline{617470}$ & IL18BP & Interleukin 18 binding protein & $138 \uparrow$ & 0.001 \\
\hline 10029678 & LOC10029678 & Immediate early response 3 interacting protein 1 pseudogene & $138 \uparrow$ & 0.001 \\
\hline$\underline{531606}$ & SRXN1 & Sulfiredoxin 1 & $137.5 \uparrow$ & 0.002 \\
\hline$\underline{515042}$ & LOC515042 & SCAN domain containing 1-like & $137.3 \uparrow$ & $<0.001$ \\
\hline$\underline{509956}$ & CTSG & Cathepsin G & $136.1 \uparrow$ & $<0.001$ \\
\hline$\underline{616906}$ & EIF4E3 & Eukaryotic translation initiation factor $4 \mathrm{E}$ family member 3 & $136.1 \uparrow$ & $<0.001$ \\
\hline 539034 & PFN2 & Profilin 2 & $135.7 \uparrow$ & 0.003 \\
\hline
\end{tabular}


Table 6.7. Supplemental (continued)

\begin{tabular}{|c|c|c|c|c|}
\hline Gene ID & Symbol & Full name & $\begin{array}{l}\text { Percent } \\
\text { change }^{1}\end{array}$ & P-Value \\
\hline 532573 & FLOT1 & Flotillin 1 & $134.7 \uparrow$ & $<0.001$ \\
\hline 767964 & CXHXorf65 & Chromosome $\mathrm{X}$ open reading frame, human cxorf65 & $131.9 \uparrow$ & 0.001 \\
\hline
\end{tabular}

\begin{tabular}{|c|c|c|c|c|}
\hline$\underline{618294}$ & IL9R & Interleukin 9 receptor & $88.7 \downarrow$ & $<0.001$ \\
\hline 337885 & GPR44 & G protein-coupled receptor 44 & $85.2 \downarrow$ & $<0.001$ \\
\hline$\underline{516066}$ & GATA1 & GATA binding protein 1 (globin transcription factor 1 ) & $84.5 \downarrow$ & $<0.001$ \\
\hline$\underline{514271}$ & TMEM106C & Transmembrane protein $106 \mathrm{C}$ & $82.9 \downarrow$ & 0.001 \\
\hline$\underline{512578}$ & KCTD15 & Potassium channel tetramerisation domain containing 15 & $80.7 \downarrow$ & $<0.001$ \\
\hline$\underline{10033550}$ & LOC10033550 & Zinc finger protein 652 -like & $79.9 \downarrow$ & $<0.001$ \\
\hline$\underline{540387}$ & CYSLTR2 & Cysteinyl leukotriene receptor 2 & $78.8 \downarrow$ & $<0.001$ \\
\hline$\underline{10029704}$ & LOC10029704 & Chemokine (C-C motif) ligand 14-like & $77.9 \downarrow$ & $<0.001$ \\
\hline$\underline{506809}$ & GATA2 & GATA binding protein 2 & $77.4 \downarrow$ & 0.009 \\
\hline$\underline{514745}$ & DHCR7 & 7-dehydrocholesterol reductase & $76.8 \downarrow$ & $<0.001$ \\
\hline$\underline{783354}$ & HRH4 & Histamine receptor $\mathrm{H} 4$ & $76.2 \downarrow$ & $<0.001$ \\
\hline$\underline{537773}$ & GFI1B & Growth factor independent 1B transcription repressor & $75.9 \downarrow$ & $<0.001$ \\
\hline$\underline{404172}$ & PTI & Pancreatic trypsin inhibitor & $75.4 \downarrow$ & $<0.001$ \\
\hline$\underline{514201}$ & SMPD3 & Sphingomyelin phosphodiesterase 3 , neutral membrane (neutral sphingomyelinase & $74.9 \downarrow$ & $<0.001$ \\
\hline$\underline{281286}$ & LYL1 & Lymphoblastic leukemia derived sequence 1 & $74.5 \downarrow$ & $<0.001$ \\
\hline$\underline{506281}$ & IDO1 & Indoleamine 2,3-dioxygenase 1 & $74.2 \downarrow$ & $<0.001$ \\
\hline$\overline{782309}$ & PLEKHF1 & Pleckstrin homology domain containing, family F (with FYVE domain) member 1 & $74.2 \downarrow$ & $<0.001$ \\
\hline$\underline{10029648}$ & GLOD5 & Glyoxalase domain containing 5 & $73.4 \downarrow$ & $<0.001$ \\
\hline$\underline{281156}$ & FDPS & Farnesyl diphosphate synthase & $73.3 \downarrow$ & $<0.001$ \\
\hline 282139 & ALOX15 & Arachidonate 15-lipoxygenase & $73 \downarrow$ & $<0.001$ \\
\hline$\underline{10013945}$ & LOC10013945 & Rab37, member RAS oncogene family-like & $73 \downarrow$ & $<0.001$ \\
\hline$\underline{514003}$ & CDC40 & Cell division cycle 40 homolog (S. Cerevisiae) & $72.6 \downarrow$ & $<0.001$ \\
\hline
\end{tabular}


Table 6.7. Supplemental (continued)

\begin{tabular}{|c|c|c|c|c|}
\hline Gene ID & Symbol & Full name & $\begin{array}{l}\text { Percent } \\
\text { change }\end{array}$ & P-Value \\
\hline 10033647 & LOC10033647 & Colony stimulating factor 2 receptor, alpha, low-affinity (granulocyte- & $71.9 \downarrow$ & $<0.001$ \\
\hline 505056 & GPR35 & G protein-coupled receptor 35 & $71.6 \downarrow$ & $<0.001$ \\
\hline 338437 & SOCS2 & Suppressor of cytokine signaling 2 & $71.4 \downarrow$ & $<0.001$ \\
\hline$\overline{784903}$ & $\mathrm{C} 21 \mathrm{H} 15$ orf 39 & Chromosome 21 open reading frame, human c15orf39 & $71.3 \downarrow$ & $<0.001$ \\
\hline 10033640 & LOC10033640 & Transport and golgi organization 2-like & $71 \downarrow$ & $<0.001$ \\
\hline 539107 & GPR55 & G protein-coupled receptor 55 & $70.7 \downarrow$ & $<0.001$ \\
\hline 536427 & $\mathrm{CCDC} 42$ & Coiled-coil domain containing 42 & $70.5 \downarrow$ & $<0.001$ \\
\hline$\underline{513777}$ & CCDC104 & Coiled-coil domain containing 104 & $70.4 \downarrow$ & $<0.001$ \\
\hline$\overline{404103}$ & LOC404103 & Spleen trypsin inhibitor & $70.3 \downarrow$ & 0.001 \\
\hline$\overline{506294}$ & KIF22 & Kinesin family member 22 & $69.7 \downarrow$ & $<0.001$ \\
\hline$\overline{532550}$ & BRAT1 & BRCA1-associated ATM activator 1 & $69.5 \downarrow$ & $<0.001$ \\
\hline$\overline{520515}$ & CRAMP1L & Crm, cramped-like (Drosophila) & $69.5 \downarrow$ & $<0.001$ \\
\hline$\overline{539466}$ & PPP1R3C & Protein phosphatase 1 , regulatory subunit $3 \mathrm{C}$ & $69.5 \downarrow$ & $<0.001$ \\
\hline$\overline{788039}$ & ARL4C & ADP-ribosylation factor-like $4 \mathrm{C}$ & $69.3 \downarrow$ & $<0.001$ \\
\hline$\underline{537224}$ & LPIN1 & Lipin 1 & $69 \downarrow$ & $<0.001$ \\
\hline$\underline{512222}$ & PDE4A & Phosphodiesterase 4A, camp-specific & $68.5 \downarrow$ & $<0.001$ \\
\hline$\underline{618296}$ & POLR3K & Polymerase (RNA) III (DNA directed) polypeptide K, $12.3 \mathrm{kda}$ & $68.1 \downarrow$ & $<0.001$ \\
\hline$\underline{782045}$ & CYSLTR1 & Cysteinyl leukotriene receptor 1 & $66.9 \downarrow$ & $<0.001$ \\
\hline$\overline{539146}$ & GPR132 & G protein-coupled receptor 132 & $66.9 \downarrow$ & $<0.001$ \\
\hline$\underline{509974}$ & GPR114 & G protein-coupled receptor 114 & $66.8 \downarrow$ & $<0.001$ \\
\hline$\underline{539244}$ & ASB2 & Ankyrin repeat and SOCS box containing 2 & $66.1 \downarrow$ & $<0.001$ \\
\hline$\overline{504771}$ & COQ7 & Coenzyme Q7 homolog, ubiquinone (yeast) & $66 \downarrow$ & $<0.001$ \\
\hline$\overline{785599}$ & $\mathrm{ZCCHC} 13$ & Zinc finger protein 9 & $66 \downarrow$ & $<0.001$ \\
\hline$\underline{614376}$ & LOC614376 & Histone cluster 2 , h2be-like & $66 \downarrow$ & 0.001 \\
\hline$\underline{514616}$ & DOK2 & Docking protein $2,56 \mathrm{kda}$ & $65.8 \downarrow$ & $<0.001$ \\
\hline 786191 & HIC1 & Hypermethylated in cancer 1 protein-like & $65.8 \downarrow$ & 0.003 \\
\hline
\end{tabular}


Table 6.7. Supplemental (continued)

\begin{tabular}{|c|c|c|c|c|}
\hline Gene ID & Symbol & Full name & $\begin{array}{l}\text { Percent } \\
\text { change }\end{array}$ & P-Value \\
\hline 617118 & DNAJC30 & Dnaj (Hsp40) homolog, subfamily C, member 30 & $65.6 \downarrow$ & $<0.001$ \\
\hline$\overline{526835}$ & NEURL2 & Neuralized homolog 2 (Drosophila) & $65.6 \downarrow$ & 0.001 \\
\hline 280707 & $\mathrm{TH}$ & Tyrosine hydroxylase & $64.6 \downarrow$ & $<0.001$ \\
\hline 783487 & ENHO & Energy homeostasis associated & $64.5 \downarrow$ & $<0.001$ \\
\hline 10029769 & LOC10029769 & Uncharacterized LOC100297694 & $64.1 \downarrow$ & $<0.001$ \\
\hline$\underline{504481}$ & MSMO1 & Methylsterol monooxygenase 1 & $64.1 \downarrow$ & $<0.001$ \\
\hline 281718 & CRYAA & Crystallin, alpha A & $64.1 \downarrow$ & 0.001 \\
\hline$\overline{518114}$ & GPC1 & Glypican 1 & $63.8 \downarrow$ & $<0.001$ \\
\hline$\overline{791010}$ & MIR142 & Microrna mir-142 & $63.6 \downarrow$ & 0.002 \\
\hline$\overline{511899}$ & INSIG1 & Insulin induced gene 1 & $63.4 \downarrow$ & $<0.001$ \\
\hline$\overline{511792}$ & HPS6 & Hermansky-Pudlak syndrome 6 & $63.2 \downarrow$ & $<0.001$ \\
\hline$\overline{616928}$ & $\mathrm{C} 16 \mathrm{H} 1$ orf 186 & Chromosome 16 open reading frame, human c1orf186 & $63.1 \downarrow$ & $<0.001$ \\
\hline 282010 & PSEN2 & Presenilin 2 (Alzheimer disease 4) & $63 \downarrow$ & $<0.001$ \\
\hline 513131 & CTBP1 & C-terminal binding protein 1 & $63 \downarrow$ & 0.002 \\
\hline 514293 & IDI1 & Isopentenyl-diphosphate delta isomerase 1 & $62.8 \downarrow$ & $<0.001$ \\
\hline 10013789 & LGALS9 & Lectin, galactoside-binding, soluble, 9 & $62.8 \downarrow$ & $<0.001$ \\
\hline$\underline{528767}$ & LOC528767 & Kinesin family member 19-like & $62.8 \downarrow$ & $<0.001$ \\
\hline$\overline{515828}$ & LOC515828 & Uncharacterized LOC515828 & $62.5 \downarrow$ & $<0.001$ \\
\hline 10029659 & LOC10029659 & Uncharacterized LOC100296596 & $62.5 \downarrow$ & 0.005 \\
\hline$\underline{615906}$ & LSS & Lanosterol synthase (2,3-oxidosqualene-lanosterol cyclase) & $62.4 \downarrow$ & $<0.001$ \\
\hline$\underline{767866}$ & NOTCH1 & Notch 1 & $62.4 \downarrow$ & 0.001 \\
\hline$\overline{510745}$ & ABCG1 & ATP-binding cassette, sub-family G (WHITE), member 1 & $62.2 \downarrow$ & $<0.001$ \\
\hline$\underline{615712}$ & EXOSC2 & Exosome component 2 & $62.1 \downarrow$ & $<0.001$ \\
\hline$\underline{538173}$ & PGP & Phosphoglycolate phosphatase & $62.1 \downarrow$ & $<0.001$ \\
\hline$\underline{615848}$ & IL5RA & Interleukin 5 receptor, alpha & $61.8 \downarrow$ & $<0.001$ \\
\hline 10014041 & LOC10014041 & Uncharacterized LOC100140410 & $61.6 \downarrow$ & $<0.001$ \\
\hline
\end{tabular}


Table 6.7. Supplemental (continued)

\begin{tabular}{|c|c|c|c|c|}
\hline Gene ID & Symbol & Full name & $\begin{array}{l}\text { Percent } \\
\text { change }^{1}\end{array}$ & P-Value \\
\hline$\underline{509859}$ & TRANK1 & Tetratricopeptide repeat and ankyrin repeat containing 1 & $61.6 \downarrow$ & 0.001 \\
\hline$\overline{511504}$ & CAMK1G & Calcium/calmodulin-dependent protein kinase IG & $61.5 \downarrow$ & $<0.001$ \\
\hline$\underline{281276}$ & LDLR & Low density lipoprotein receptor & $61.4 \downarrow$ & $<0.001$ \\
\hline$\overline{532244}$ & MICAL3 & Microtubule associated monoxygenase, calponin and LIM domain containing 3 & $61.4 \downarrow$ & $<0.001$ \\
\hline$\underline{3283879}$ & COX1 & Cytochrome c oxidase subunit I & $61.3 \downarrow$ & 0.001 \\
\hline$\underline{505792}$ & MVK & Mevalonate kinase & $61.2 \downarrow$ & $<0.001$ \\
\hline 407234 & ADIPOR2 & Adiponectin receptor 2 & $61.1 \downarrow$ & $<0.001$ \\
\hline$\underline{512243}$ & ZSWIM1 & Zinc finger, SWIM-type containing 1 & $61.1 \downarrow$ & 0.001 \\
\hline$\underline{511144}$ & DDRGK1 & DDRGK domain containing 1 & $61 \downarrow$ & $<0.001$ \\
\hline 783439 & C3H1orf 226 & Chromosome 3 open reading frame, human c1orf 226 & $60.7 \downarrow$ & $<0.001$ \\
\hline$\underline{539159}$ & CTH & Cystathionase (cystathionine gamma-lyase) & $60.6 \downarrow$ & $<0.001$ \\
\hline$\underline{540287}$ & GPR183 & G protein-coupled receptor 183 & $60.2 \downarrow$ & $<0.001$ \\
\hline$\underline{526535}$ & SQLE & Squalene epoxidase & $60.1 \downarrow$ & $<0.001$ \\
\hline$\underline{613856}$ & LY86 & Lymphocyte antigen 86 & $60 \downarrow$ & $<0.001$ \\
\hline$\underline{540067}$ & EPM2AIP1 & EPM2A (laforin) interacting protein 1 & $59.9 \downarrow$ & 0.004 \\
\hline$\underline{614645}$ & SPN & Sialophorin & $59.8 \downarrow$ & $<0.001$ \\
\hline$\underline{618217}$ & LOC618217 & Zinc finger protein 316-like & $59.7 \downarrow$ & 0.001 \\
\hline$\underline{282493}$ & HLA-DOB & Major histocompatibility complex, class II, DO beta & $59.6 \downarrow$ & $<0.001$ \\
\hline$\underline{511206}$ & MPO & Myeloperoxidase & $59.6 \downarrow$ & $<0.001$ \\
\hline$\underline{617636}$ & MMAB & Methylmalonic aciduria (cobalamin deficiency) cblb type & $59.6 \downarrow$ & 0.001 \\
\hline$\underline{535236}$ & MLST8 & MTOR associated protein, LST8 homolog (S. Cerevisiae) & $59.5 \downarrow$ & $<0.001$ \\
\hline$\overline{533726}$ & DHCR24 & 24-dehydrocholesterol reductase & $59.4 \downarrow$ & $<0.001$ \\
\hline$\underline{511523}$ & SLC2A4RG & SLC2A4 regulator & $59.4 \downarrow$ & $<0.001$ \\
\hline$\underline{507878}$ & SCAP & SREBF chaperone & $59.4 \downarrow$ & $<0.001$ \\
\hline$\underline{517002}$ & ACP5 & Acid phosphatase 5 , tartrate resistant & $59.3 \downarrow$ & $<0.001$ \\
\hline$\underline{505060}$ & CYP51A1 & Cytochrome P450, family 51, subfamily A, polypeptide 1 & $59.3 \downarrow$ & $<0.001$ \\
\hline
\end{tabular}


Table 6.7. Supplemental (continued)

\begin{tabular}{llll}
\hline Gene ID & Symbol & Full name & $\begin{array}{c}\text { Percent } \\
\text { change }^{1}\end{array}$ P-Value \\
\hline
\end{tabular}

Effect of LPS (50 vs. $0 \mu \mathrm{g} / \mathrm{ml}$ ) in PMNL from different physiolocial states (postpartum vs. Prepartum) ${ }^{2}$

Up-regulated

$\underline{540198}$ OXER1 Oxoeicosanoid (OXE) receptor 1

$4671.6 \uparrow \quad 0.008$

Down-regulated

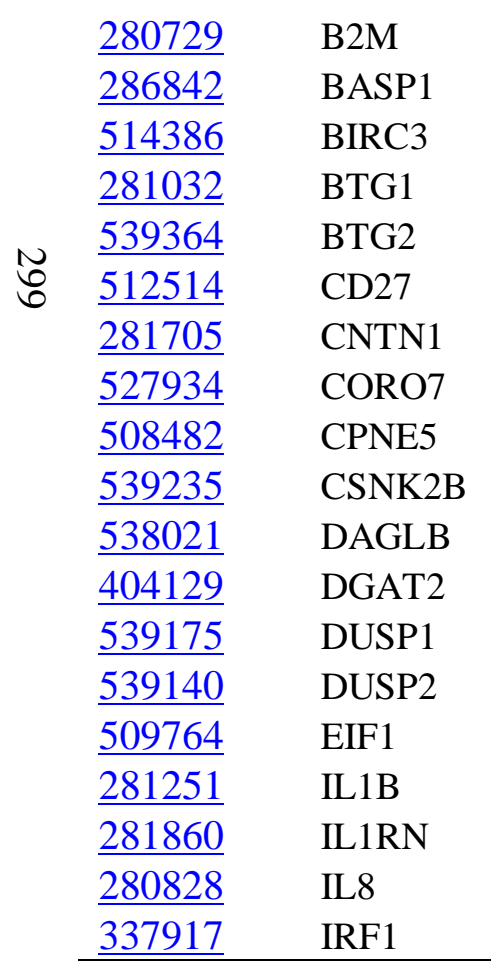

Beta-2-microglobulin

Brain abundant, membrane attached signal protein 1

Baculoviral IAP repeat containing 3

B-cell translocation gene 1, anti-proliferative

BTG family, member 2

CD27 molecule

Contactin 1

Coronin 7

Copine V

Casein kinase 2, beta polypeptide

Diacylglycerol lipase, beta

Diacylglycerol O-acyltransferase 2

Dual specificity phosphatase 1

Dual specificity phosphatase 2

Eukaryotic translation initiation factor 1

Interleukin 1, beta

Interleukin 1 receptor antagonist

Interleukin 8

Interferon regulatory factor 1

$\begin{aligned} 100 \downarrow & <0.001 \\ 100 \downarrow & <0.001 \\ 100 \downarrow & <0.001 \\ 100 \downarrow & <0.001 \\ 100 \downarrow & <0.001 \\ 100 \downarrow & <0.001 \\ 100 \downarrow & <0.001 \\ 100 \downarrow & <0.001 \\ 100 \downarrow & <0.001 \\ 100 \downarrow & <0.001 \\ 100 \downarrow & <0.001 \\ 100 \downarrow & <0.001 \\ 100 \downarrow & <0.001 \\ 100 \downarrow & <0.001 \\ 100 \downarrow & <0.001 \\ 100 \downarrow & <0.001 \\ 100 \downarrow & <0.001 \\ 100 \downarrow & <0.001 \\ 100 \downarrow & <0.001\end{aligned}$


Table 6.7. Supplemental (continued)

\begin{tabular}{|c|c|c|c|c|}
\hline Gene ID & Symbol & Full name & $\begin{array}{l}\text { Percent } \\
\text { change }^{1}\end{array}$ & P-Value \\
\hline 504806 & IRG1 & Immunoresponsive 1 homolog (mouse) & $100 \downarrow$ & $<0.001$ \\
\hline 514246 & JUNB & Jun B proto-oncogene & $100 \downarrow$ & $<0.001$ \\
\hline 522795 & KLF10 & Kruppel-like factor 10 & $100 \downarrow$ & $<0.001$ \\
\hline 10013849 & LOC10013849 & Uncharacterized LOC100138493 & $100 \downarrow$ & $<0.001$ \\
\hline$\overline{10029659}$ & LOC10029659 & Uncharacterized LOC100296593 & $100 \downarrow$ & $<0.001$ \\
\hline 281370 & UBB & Ubiquitin B & $100 \downarrow$ & $<0.001$ \\
\hline$\overline{508666}$ & LOC508666 & C-C motif chemokine 23 & $100 \downarrow$ & $<0.001$ \\
\hline 529195 & MEFV & Mediterranean fever & $100 \downarrow$ & $<0.001$ \\
\hline 517231 & KIAA0226L & KIAA0226-like ortholog & $100 \downarrow$ & $<0.001$ \\
\hline$\underline{514626}$ & MXD1 & MAX dimerization protein 1 & $100 \downarrow$ & $<0.001$ \\
\hline$\underline{539780}$ & NAIP & NLR family, apoptosis inhibitory protein & $100 \downarrow$ & $<0.001$ \\
\hline 281345 & NCF1 & Neutrophil cytosolic factor 1 & $100 \downarrow$ & $<0.001$ \\
\hline 282291 & NFKBIA & Nuclear factor of kappa light polypeptide gene enhancer in B-cells inhibitor, alpha & $100 \downarrow$ & $<0.001$ \\
\hline 282713 & NFKBIZ & Nuclear factor of kappa light polypeptide gene enhancer in B-cells inhibitor, zeta & $100 \downarrow$ & $<0.001$ \\
\hline$\underline{10012450}$ & PDE4B & Phosphodiesterase 4B, camp-specific & $100 \downarrow$ & $<0.001$ \\
\hline$\underline{281983}$ & PLAUR & Plasminogen activator, urokinase receptor & $100 \downarrow$ & $<0.001$ \\
\hline 518658 & PLEK & Pleckstrin & $100 \downarrow$ & $<0.001$ \\
\hline$\underline{513055}$ & RGS2 & Regulator of G-protein signaling 2, 24kda & $100 \downarrow$ & $<0.001$ \\
\hline$\underline{534171}$ & SAMSN1 & SAM domain, SH3 domain and nuclear localization signals 1 & $100 \downarrow$ & $<0.001$ \\
\hline$\underline{508861}$ & SAT1 & Spermidine/spermine N1-acetyltransferase 1 & $100 \downarrow$ & $<0.001$ \\
\hline$\underline{514346}$ & SDS & Serine dehydratase & $100 \downarrow$ & $<0.001$ \\
\hline 281485 & SELL & Selectin L & $100 \downarrow$ & $<0.001$ \\
\hline 510239 & SEMA4A & Sema domain, immunoglobulin domain (Ig), transmembrane domain (TM) and & $100 \downarrow$ & $<0.001$ \\
\hline 281375 & SERPINE1 & Serpin peptidase inhibitor, clade E (nexin, plasminogen activator inhibitor type 1), & $100 \downarrow$ & $<0.001$ \\
\hline$\underline{515854}$ & SGK1 & Serum/glucocorticoid regulated kinase 1 & $100 \downarrow$ & $<0.001$ \\
\hline$\underline{327666}$ & SIRPA & Signal-regulatory protein alpha & $100 \downarrow$ & $<0.001$ \\
\hline
\end{tabular}


Table 6.7. Supplemental (continued)

\begin{tabular}{|c|c|c|c|c|}
\hline Gene ID & Symbol & Full name & $\begin{array}{l}\text { Percent } \\
\text { change }^{1}\end{array}$ & P-Value \\
\hline 282358 & SLC2A3 & Solute carrier family 2 (facilitated glucose transporter), member 3 & $100 \downarrow$ & $<0.001$ \\
\hline$\underline{533166}$ & SORL1 & Sortilin-related receptor, L(DLR class) A repeats containing & $100 \downarrow$ & $<0.001$ \\
\hline 509501 & SRGN & Serglycin & $100 \downarrow$ & $<0.001$ \\
\hline$\overline{505080}$ & TGM3 & Transglutaminase 3 (E polypeptide, protein-glutamine-gamma-glutamyltransferase) & $100 \downarrow$ & $<0.001$ \\
\hline$\underline{508105}$ & TNFAIP3 & Tumor necrosis factor, alpha-induced protein 3 & $100 \downarrow$ & $<0.001$ \\
\hline$\overline{526103}$ & TNIP1 & TNFAIP3 interacting protein 1 & $100 \downarrow$ & $<0.001$ \\
\hline 506790 & TXNIP & Thioredoxin interacting protein & $100 \downarrow$ & $<0.001$ \\
\hline 444874 & UBC & Ubiquitin C & $100 \downarrow$ & $<0.001$ \\
\hline$\underline{534929}$ & VNN2 & Vanin 2 & $100 \downarrow$ & $<0.001$ \\
\hline$\underline{280960}$ & $\mathrm{XDH}$ & Xanthine dehydrogenase & $100 \downarrow$ & $<0.001$ \\
\hline$\underline{282127}$ & ZFP36 & Zinc finger protein $36, \mathrm{C} 3 \mathrm{H}$ type, homolog (mouse) & $100 \downarrow$ & $<0.001$ \\
\hline 527889 & CSNK1G1 & Casein kinase 1 , gamma 1 & $100 \downarrow$ & 0.001 \\
\hline$\underline{507417}$ & ABTB1 & Ankyrin repeat and BTB (POZ) domain containing 1 & $99.9 \downarrow$ & $<0.001$ \\
\hline$\underline{536628}$ & ACSL4 & Acyl-coa synthetase long-chain family member 4 & $99.9 \downarrow$ & $<0.001$ \\
\hline 280979 & ACTB & Actin, beta & $99.9 \downarrow$ & $<0.001$ \\
\hline 510907 & ADAM19 & ADAM metallopeptidase domain 19 & $99.9 \downarrow$ & $<0.001$ \\
\hline 504288 & AKAP13 & A kinase (PRKA) anchor protein 13 & $99.9 \downarrow$ & $<0.001$ \\
\hline$\underline{509575}$ & AMICA1 & Adhesion molecule, interacts with CXADR antigen 1 & $99.9 \downarrow$ & $<0.001$ \\
\hline$\underline{519803}$ & AMPD3 & Adenosine monophosphate deaminase 3 & $99.9 \downarrow$ & $<0.001$ \\
\hline$\underline{532856}$ & ANKRD11 & Ankyrin repeat domain 11 & $99.9 \downarrow$ & $<0.001$ \\
\hline$\overline{510080}$ & ANTXR2 & Anthrax toxin receptor 2 & $99.9 \downarrow$ & $<0.001$ \\
\hline 327662 & ANXA1 & Annexin A1 & $99.9 \downarrow$ & $<0.001$ \\
\hline 518752 & ARG2 & Arginase, type II & $99.9 \downarrow$ & $<0.001$ \\
\hline$\overline{538835}$ & ARHGAP30 & Rho gtpase activating protein 30 & $99.9 \downarrow$ & $<0.001$ \\
\hline$\overline{538517}$ & ARRDC4 & Arrestin domain containing 4 & $99.9 \downarrow$ & $<0.001$ \\
\hline 508723 & ATP8A2 & Atpase, aminophospholipid transporter, class I, type $8 \mathrm{~A}$, member 2 & $99.9 \downarrow$ & $<0.001$ \\
\hline
\end{tabular}


Table 6.7. Supplemental (continued)

\begin{tabular}{|c|c|c|c|c|}
\hline Gene ID & Symbol & Full name & $\begin{array}{l}\text { Percent } \\
\text { change }^{1}\end{array}$ & P-Value \\
\hline 531942 & BCL3 & B-cell CLL/lymphoma 3 & $99.9 \downarrow$ & $<0.001$ \\
\hline 505676 & BOLA & MHC class I heavy chain & $99.9 \downarrow$ & $<0.001$ \\
\hline 510417 & BOLA-NC1 & Non-classical MHC class I antigen & $99.9 \downarrow$ & $<0.001$ \\
\hline 10012604 & BOLA-N & MHC class I antigen & $99.9 \downarrow$ & $<0.001$ \\
\hline 510417 & BOLA-NC1 & Non-classical MHC class I antigen & $99.9 \downarrow$ & $<0.001$ \\
\hline 512628 & C21H14orf79 & Chromosome 21 open reading frame, human c14orf79 & $99.9 \downarrow$ & $<0.001$ \\
\hline 282412 & CACNA1H & Calcium channel, voltage-dependent, $\mathrm{T}$ type, alpha $1 \mathrm{H}$ subunit & $99.9 \downarrow$ & $<0.001$ \\
\hline 532940 & CCDC28A & Coiled-coil domain containing 28A & $99.9 \downarrow$ & $<0.001$ \\
\hline$\underline{511112}$ & CCL21 & Chemokine (C-C motif) ligand 21 & $99.9 \downarrow$ & $<0.001$ \\
\hline 281668 & CCNB2 & Cyclin B2 & $99.9 \downarrow$ & $<0.001$ \\
\hline$\underline{509717}$ & $\mathrm{CCNC}$ & Cyclin C & $99.9 \downarrow$ & $<0.001$ \\
\hline 505200 & $\mathrm{CCNF}$ & Cyclin F & $99.9 \downarrow$ & $<0.001$ \\
\hline$\underline{520428}$ & CDC14B & CDC14 cell division cycle 14 homolog B (S. Cerevisiae) & $99.9 \downarrow$ & $<0.001$ \\
\hline 404089 & CLDN2 & Claudin 2 & $99.9 \downarrow$ & $<0.001$ \\
\hline 540938 & CNOT10 & CCR4-NOT transcription complex, subunit 10 & $99.9 \downarrow$ & $<0.001$ \\
\hline$\underline{526697}$ & CNTN3 & Contactin 3 (plasmacytoma associated) & $99.9 \downarrow$ & $<0.001$ \\
\hline 614728 & COASY & Coa synthase & $99.9 \downarrow$ & $<0.001$ \\
\hline 282419 & CSNK2A1 & Casein kinase 2 , alpha 1 polypeptide & $99.9 \downarrow$ & $<0.001$ \\
\hline$\underline{540286}$ & DAZAP2 & DAZ associated protein 2 & $99.9 \downarrow$ & $<0.001$ \\
\hline$\underline{529235}$ & DDIT4 & DNA-damage-inducible transcript 4 & $99.9 \downarrow$ & $<0.001$ \\
\hline$\underline{614215}$ & DDX39A & DEAD (Asp-Glu-Ala-Asp) box polypeptide 39A & $99.9 \downarrow$ & $<0.001$ \\
\hline$\underline{510093}$ & DDX3X & DEAD (Asp-Glu-Ala-Asp) box polypeptide 3, X-linked & $99.9 \downarrow$ & $<0.001$ \\
\hline$\underline{533700}$ & DDX5 & DEAD (Asp-Glu-Ala-Asp) box polypeptide 5 & $99.9 \downarrow$ & $<0.001$ \\
\hline$\overline{513906}$ & DDX6 & DEAD (Asp-Glu-Ala-Asp) box polypeptide 6 & $99.9 \downarrow$ & $<0.001$ \\
\hline$\underline{534251}$ & DEDD2 & Death effector domain containing 2 & $99.9 \downarrow$ & $<0.001$ \\
\hline 784484 & DENND5A & DENN/MADD domain containing 5A & $99.9 \downarrow$ & $<0.001$ \\
\hline
\end{tabular}


Table 6.7. Supplemental (continued)

\begin{tabular}{|c|c|c|c|c|}
\hline Gene ID & Symbol & Full name & $\begin{array}{l}\text { Percent } \\
\text { change }^{1}\end{array}$ & P-Value \\
\hline 511691 & DNM2 & Dynamin 2 & $99.9 \downarrow$ & $<0.001$ \\
\hline 281133 & ECE1 & Endothelin converting enzyme 1 & $99.9 \downarrow$ & $<0.001$ \\
\hline 514259 & EFHD2 & EF-hand domain family, member D2 & $99.9 \downarrow$ & $<0.001$ \\
\hline
\end{tabular}

${ }^{1}$ Direction of change in expression of genes is indicated with a $\uparrow$ or $\downarrow$.

${ }^{2}$ Neutrophils were collected on days $58.1 \pm 1.0$ prepartum (pre-treatment sample) and $6.4 \pm 0.1$ postpartum from cows fed a basal diet with no added dietary trace minerals (basal, $\mathrm{n}=7$ ) or supplemented with $200 \mathrm{mg} \mathrm{Mn,} 25 \mathrm{mg} \mathrm{Co,} 125 \mathrm{mg} \mathrm{Cu}$, and $360 \mathrm{mg} \mathrm{Zn}$ from sulfate and carbonate sources (inorganic, $\mathrm{n}=7$ ) or supplied by amino acid complexes of $\mathrm{Mn}, \mathrm{Cu}$, and $\mathrm{Zn}$, and cobalt glucoheptonate (organic, $\mathrm{n}=7$ ) after incubation with 0 or $50 \mu \mathrm{g} / \mathrm{mL}$ of $E$. coli lipopolysaccharide for $120 \mathrm{~min}$. 


\section{CHAPTER SEVEN}

\section{INTREGRATED DISCUSSION FROM THE STUDY OF NUTRITIONAL TACTICS TO IMPROVE INNATE IMMUNITY IN DAIRY COWS}

The experiments described in Chapters 2 through 6 have contributed to our knowledge in two major areas of research in bovine innate immunity: (1) neutrophil (PMNL) biology during the periparturient period; and (2) potential nutritional strategies to prevent the decline in immune competence typical of this time. In Chapter 2, incubation with Escherichia coli lipopolysaccharide (LPS) stimulated the production of reactive oxygen species (ROS) and release of extracellular traps (NETs) by neutrophils, but did not alter their chemotaxis or killing against Staphylococcus aureus. Macrophages and epithelial cells are considered the primary cells involved in the recognition of LPS and synthesis of pro-inflammatory cytokines (Burvenich et al., 2007). However, our findings pointed out that PMNL are capable of recognizing LPS directly and activate some of their antimicrobial weapons in response to this stimulus. Furthermore, these results provided the experimental layout for the in vitro experiments conducted in Chapter 5 in which the function of neutrophils was assessed in both resting and LPSactivated states. Although the source of trace mineral supplementation did not influence the function of neutrophils treated with 0 or $50 \mu \mathrm{g} / \mathrm{mL}$ of LPS in Chapter 5 , it was confirmed that LPS increases NETs release by non-activated cells. This is the first report of release of NETs by bovine PMNL in response to LPS following the discovery of this novel antimicrobial mechanism in human PMNL by Brinkmann et al. (2004). In Chapter 2, RT-PCR was then used to determine the effects of LPS on genes encoding for 
cytokines and proteins involved in ROS production. In agreement with other studies (Worku and Morris, 2009; Yu et al., 2010), a potent increase in the contents of interleukin-8 (IL-8) and tumor necrosis factor- $\alpha$ (TNF) mRNA after a 2-hour incubation with LPS was detected. It was also reported for the first time that LPS induces the expression of superoxide dismutase 2 (SOD2). This later finding provided a link between gene expression and cellular function as SOD2 is one of the enzymes involved in the production of ROS. Changes in PMNL global gene expression in response to LPS were further investigated in Chapter 4 using RNA-sequencing. Advantages of using this emerging technology over microarrays to study gene expression include improved sensitivity, low background signal, and independency from pre-existing assumptions about the genome (Wang et al., 2009a). The PMNL used in this experiment were collected from cows receiving $56 \mathrm{~g}$ of either a carrier or a B-vitamin supplement. Regardless of the effect of dietary supplementation, a wide repertoire of LPS-responsive genes in bovine PMNL were further identified. Up-regulated genes due to LPS included pro-inflammatory cytokines and other molecules involved in the innate immune response. Gene ontology analysis revealed enriched pathways consistent with immune activation including the cytokine-cytokine receptor interaction, hematopoietic cell lineage, and JakSTAT signaling pathways. Within the cytokine-cytokine receptor interaction pathway, LPS increased the mRNA expression of IL-8 and TNF as previously reported in Chapter 2. In addition, several cytokines such as IL-1A, IL-1B, IL-5, IL-12, IL-21A and their receptors IL-8RA, IL-8RB, IL-5RA, and IL-1R2 were up-regulated by incubation with 50 $\mu \mathrm{g} / \mathrm{mL}$ of LPS. 
In the experiments in Chapters 2, 3, and 5, the functional capacity of PMNL and expression of relevant genes was measured on different time points during the periparturient period and midlactation. Overall, our findings confirm a reduced PMNL antimicrobial performance during the periparturient period. Altered PMNL function in periparturient dairy cows has been previously reported by several groups including our laboratory (Revelo and Waldron, 2010). In Chapter 2 for instance, RT-PCR was used to determine the expression of LPS-responsive genes in PMNL collected from early lactation and midlactation cows. Independent of LPS treatment, PMNL from early lactation cows had lower mRNA contents of tumor necrosis factor- $\alpha$ (TNF) and interleukin-8 (IL-8), compared with PMNL from midlactation cows. Also, LPS stimulated the expression of TNF, bactericidal/permeability-increasing protein (BPI), and cytochrome b-245, alpha polypeptide (CYBA) only in PMNL from midlactation cows. In contrast, PMNL from early lactation cows failed to respond to this LPS stimulus. These findings shed light into several potential molecular mechanisms by which PMNL may become immune-competent during the periparturient period. For example, it is possible that reduced expression of CYBA contributes to the decrease in ROS production. Additionally, low expression of IL-8 and TNF during early lactation may obstruct a vigorous immune response as these pro-inflammatory cytokines are critical during the recruitment of PMNL into the infected tissue. Consistently with these defects in the expression of selected genes, the function of PMNL was found to be altered in Chapters 3 and 5. The findings from studies of PMNL function during the periparturient period in dairy cows are summarized in Table 7.1. Most notably, neutrophils from periparturient cows produced less ROS during this time, as previously reported by several researchers 
including our group (Revelo and Waldron, 2010). A defect in oxidative burst activity has been constantly reported in the literature and has been associated directly with increased severity of coliform mastitis in dairy cows (Heyneman et al., 1990). In contrast to ROS production, the evidence supporting a reduction in other aspects of PMNL function during periparturition is less clear. For instance, several investigators have failed to detect a reduction in directed chemotaxis during the periparturient period (Kehrli and Goff, 1989; Cai et al., 1994; Detilleux et al., 1995). However, our results in Chapter 5 and those reported by Stevens et al. (2011) suggest a decrease in chemotaxis in the prepartum period. Furthermore, the majority of reports of PMNL phagocytosis and antimicrobial abilities have indicated that these measures of PMNL function either do not change or increase in the prepartum period (Table 7.1). This augmented PMNL phagocytosis during the prepartum period has been attributed to the extra energy available for phagocytic processes in a period when the consumption of energy for production of ROS is reduced (Detilleux et al., 1995). However, the increase in antimicrobial capacity prepartum observed in Chapter 5 may be more difficult to interpret as the killing ability of the PMNL should integrate all other aspects of PMNL function. Although periparturient immune-alteration is frequently defined as the period between 3 weeks on either side of parturition, most studies of PMNL function suggest that this alteration is more severe postpartum. In Chapter 6, the genetic profile of PMNL collected from prepartum (day -58 relative to parturition) and postpartum (day 6 relative to parturition) was examined using RNA-sequencing. In this experiment, PMNL from postpartum cows (vs., prepartum) had altered expression of 9,589 and 2,001 transcripts in non-activated and LPS-activated PMNL, respectively. Functional annotation analysis indicated that the chemokine 
signaling, leukocyte transendothelial migration, Fc gamma R-mediated phagocytosis, cell adhesion molecules (CAMs), TLR signaling, lysosome, apoptosis, and NOD-like receptor signaling pathways were defective in PMNL from postpartum cows. Using this high-throughput technology we identified a wide array of genes with altered expression in the postpartum period that may explain the inefficient function of PMNL during this time. 
Table 7.1. Summary of studies of neutrophil (PMNL) reactive oxygen species (ROS) production, release of extracellular traps (NETs), directed chemotaxis, killing of bacteria, and phagocytosis.

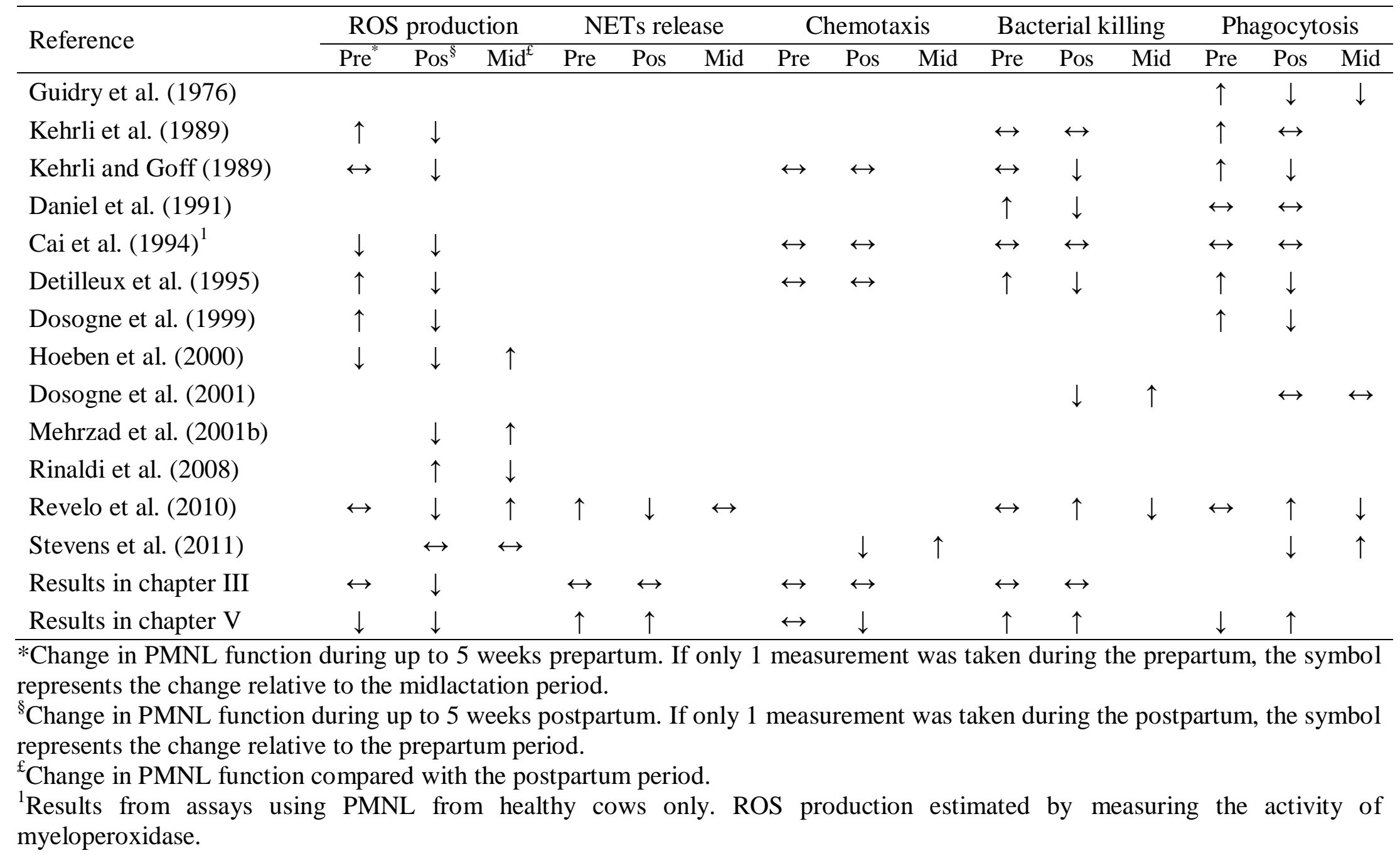


The second main objective of these studies was to investigate whether supplementation of dairy cows with certain micronutrients would prevent the decline in immune competence typical of the periparturient period. In dairy cows, insufficient dietary minerals, vitamins, and antioxidants may contribute to the altered immune function (Goff, 2006). Although the nutritional requirements of lactating dairy cows include those for minerals and vitamins (NRC, 2001), it is unknown whether these levels support optimal health. For example, the daily requirements of dairy cows for B-vitamins have been estimated based on lactating sow needs adjusted for body weight and milk production (NRC, 2001). B-vitamins are a group of water soluble vitamins involved in numerous aspects of immune function through their involvement in cellular metabolism, nucleic acid, and protein biosynthesis (Wintergerst et al., 2007). Considering the potential for B-vitamins to boost periparturient immunity; we aimed to investigate the effects of a nutritional supplement containing B-vitamins, dehydrated yeast, and trichoderma longibrachiatum fermentation products on the function and gene expression of neutrophils from periparturient cows. This nutritional supplement is commercially available as OmniGen-AF ${ }^{\circledR}$ (Prince Agri Products, Inc.) and has been reported to influence the immune response in dairy cows (Wang et al., 2009b), sheep (Wang et al., 2007), and mice (Rowson et al., 2011). In the experiment in Chapter 3, however, no differences were detected in the generation of ROS produced by the PMNL, NETs release, chemotaxis and killing of Staphylococcus aureus between PMNL collected from OmniGen-AF ${ }^{\circledR}$-supplemented and non-supplemented cows. Furthermore, in Chapter 4, we used RNA-sequencing to explore changes in PMNL gene expression in response to LPS activation in OmniGen-AF ${ }^{\circledR}$ - and non-supplemented cows. Feeding OmniGen-AF ${ }^{\circledR}$ 
to dairy cows resulted in the up- and down-regulation of 7 and 36 genes in PMNL upon activation with LPS. Pathway analysis revealed that the down-regulated genes enriched the lysosome pathway. In addition, OmniGen-AF ${ }^{\circledR}$ supplementation altered the expression of 53 transcripts, independent of LPS activation. The down-regulated genes due to this main effect of OmniGen- $\mathrm{AF}^{\circledR}$ supplementation enriched the oxidative phosphorylation pathway. Although this is purely speculative, it is possible that PMNL with lower oxidative phosphorylation activity may distribute more $\mathrm{O}_{2}$ towards effector functions with a high demand for $\mathrm{O}_{2}$ such as the production of ROS. The last finding in this study was that PMNL incubation with $50 \mu \mathrm{g} / \mathrm{mL}$ of LPS altered the expression of 333 genes, independent of dietary treatment. Incubation of PMNL with LPS enriched pathways related to immune activation such as the TLR signaling and hematopoietic cell lineage pathways. In summary, results in Chapters 3 and 4 indicate that the supplement OmniGen- $\mathrm{AF}^{\circledR}$ may not influence PMNL function, however, we revealed potential molecular mechanisms by which OmniGen- $\mathrm{AF}^{\circledR}$ may alter PMNL performance. Another group of micronutrients with potential immune-modulatory effects are the trace minerals $\mathrm{Cu}, \mathrm{Co}, \mathrm{Mn}$, and $\mathrm{Zn}$. Trace minerals are structural components and cofactors of enzymes of the antioxidant system, but can also have direct effects on immune cell function (Prasad, 2007; Spears and Weiss, 2008; Haase and Rink, 2009). Adequate trace mineral nutrition is critical for competent immune function and dietary deficiencies of these micronutrients often result in altered PMNL function. For example, induced $\mathrm{Cu}$ deficiency in cattle decreases PMNL antimicrobial capacity (Xin et al., 1991; Sharma et al., 2005), and the activity of enzymes in involved in ROS production (Cerone et al., 2000; Xin et al., 1991). Supplementation of dairy cows with trace minerals has 
conventionally relied on inorganic sources such as sulfates, oxides, and carbonates (Andrieu, 2008). However trace minerals as organic complexes (e.g., associated with amino acids or carbohydrates) have increased bioavailability compared with minerals from inorganic sources (Wedekind et al., 1992). Therefore, in Chapter 5, it was hypothesized that replacing inorganic forms of $\mathrm{Cu}, \mathrm{Co}, \mathrm{Mn}$, and $\mathrm{Zn}$ with amino acid complexes of $\mathrm{Mn}, \mathrm{Cu}$, and $\mathrm{Zn}$, and $\mathrm{Co}$ glucoheptonate would enhance PMNL function in periparturient dairy cows. The animals in this study were assigned to 3 dietary groups: no added dietary $\mathrm{Mn}, \mathrm{Co}, \mathrm{Cu}$ and $\mathrm{Zn}$ (control); additional $\mathrm{Mn}, \mathrm{Co}, \mathrm{Cu}$ and $\mathrm{Zn}$ from inorganic sources; and additional $\mathrm{Mn}, \mathrm{Co}, \mathrm{Cu}$ and $\mathrm{Zn}$ from organic sources. The control group only obtained these trace minerals from dietary forages and concentrates in the basal diet. On the other hand, the amount of trace minerals supplemented to the animals in the inorganic and organic treatment groups were the same but met or exceeded nutritional recommendations for dairy cattle (NRC, 2001). This experimental design allowed the direct comparison of trace mineral supplementation source (e.g., inorganic vs. organic) at the same level of supplementation. In this study, however, most measures of PMNL function did not differ between treatment groups except for a modest reduction in the mean fluorescence intensity (MFI) of phagocytic PMNL in non-supplemented cows. This finding indicated that the amount of phagocytized bacteria within the PMNL of cows receiving no added trace minerals is compromised. However, no differences in the MFI of phagocytic PMNL were detected between cows fed organic and inorganic trace minerals, suggesting that trace minerals from both sources perform in a similar way. Furthermore, feeding a diet devoid of added trace minerals for approximately 65 days did not affect PMNL ROS production, NETs release, chemotaxis, and antimicrobial killing of 
E. coli in periparturient dairy cows. That non-supplemented animals did not have a compelling reduction in PMNL function may indicate that these animals did not develop a severe deficiency in $\mathrm{Cu}, \mathrm{Co}, \mathrm{Mn}$, and $\mathrm{Zn}$. Therefore, it is likely that homeorhetic mechanisms were activated to ensure adequate amounts of these micronutrients dedicated towards immune function. In Chapter 6, RNA-sequencing was used to determine the potential effects of trace mineral supplementation source on the gene expression profile of PMNL. Minimal changes in PMNL gene expression were detected between cows supplemented with trace minerals, regardless of source, when compared with a basal diet containing none of these added trace minerals. This finding is consistent with the results in Chapter 5 indicating that non-supplemented cows did not have a substantial impairment of PMNL function. However, in Chapter 6, organic trace mineral supplementation, compared with that from inorganic sources, influenced the expression of 230 and 47 transcripts in non-activated and LPS-activated PMNL, respectively. As a result, we were able to identify several pathways up-regulated by organic, relative to inorganic trace mineral supplementation. This included the RIG-I-like receptor signaling, cytosolic DNA-sensing, and TLR signaling pathway. Although, organic trace mineral supplementation did not improve PMNL function in Chapter 5, the results in Chapter 6 suggest that trace minerals from organic sources (vs. inorganic) stimulate PMNL pathways involved in antigen recognition and immune response. 


\section{BIBLIOGRAPHY}

Aderem, A., and R. J. Ulevitch. 2000. Toll-like receptors in the induction of the innate immune response. Nature 406:782-787.

Aida, Y., and M. J. Pabst. 1990. Priming of neutrophils by lipopolysaccharide for enhanced release of superoxide. Requirement for plasma but not for tumor necrosis factor-alpha. J. Immunol. 145:3017-3025.

Aitken, S. L., C. M. Corl, and L. M. Sordillo. 2011. Immunopathology of mastitis: insights into disease recognition and resolution. J. Mammary Gland Biol. Neoplasia 16:291-304.

Akers, R. M., and S. C. Nickerson. 2011. Mastitis and its impact on structure and function in the ruminant mammary gland. J. Mammary Gland Biol. Neoplasia $16: 275-289$.

Akira, S., S. Uematsu, and O. Takeuchi. 2006. Pathogen recognition and innate immunity. Cell 124:783-801.

Almeida, R. A., K. R. Matthews, E. Cifrian, A. J. Guidry, and S. P. Oliver. 1996. Staphylococcus aureus invasion of bovine mammary epithelial cells. J. Dairy Sci. 79:1021-1026.

Andrieu, S. 2008. Is there a role for organic trace element supplements in transition cow health? Vet. J. 176:77-83.

Anri, A. Detection of endotoxin in affected milk from cows with coliform mastitis. Nippon Juigaku Zasshi. 1989;51:847-848.

Armitage, R. J. 1994. Tumor necrosis factor receptor superfamily members and their ligands. Curr. Opin. Immunol. 6:407-413.

Arthington, J. D., A. R. Spell, L. R. Corah, and F. Blecha. 1996. Effect of molybdenuminduced copper deficiency on in vivo and in vitro measures of neutrophil chemotaxis both before and following an inflammatory stressor. J. Anim. Sci. $74: 2759-2764$.

Asehnoune, K., D. Strassheim, S. Mitra, J. Y. Kim, and E. Abraham. 2004. Involvement of reactive oxygen species in Toll-like receptor 4-dependent activation of NFkappa B. J. Immunol. 172:2522-2529.

Auchtung, T. L., and G. E. Dahl. 2004. Prolactin mediates photoperiodic immune enhancement: effects of administration of exogenous prolactin on circulating concentrations, receptor expression, and immune function in steers. Biol. Reprod. 71:1913-1918. 
Auchtung, T. L., J. L. Salak-Johnson, D. E. Morin, C. C. Mallard, and G. E. Dahl. 2004. Effects of photoperiod during the dry period on cellular immune function of dairy cows. J. Dairy Sci. 87:3683-3689.

Ballantine, H. T., M. T. Socha, D. J. Tomlinson, A. B. Johnson, A. S. Fielding, J. K. Shearer, and S. R. van Amstel. 2002. Effect of feeding complexed zinc, manganese, copper and cobalt to late gestation and lactating dairy cows on claw integrity, reproduction and lactation performance. Prof. Anim. Sci. 18:211-218.

Bannerman, D. D. 2009. Pathogen-dependent induction of cytokines and other soluble inflammatory mediators during intramammary infection of dairy cows. J. Anim. Sci. 87:10-25.

Bannerman, D. D., M.J. Paape, W. R. Hare, E. J. Sohn. 2003. Increased levels of LPSbinding protein in bovine blood and milk following bacterial lipopolysaccharide challenge, J. Dairy Sci. 86:3128-3137.

Bannerman, D. D., M. J. Paape, J. W. Lee, X. Zhao, J. C. Hope, and P. Rainard. 2004. Escherichia coli and Staphylococcus aureus elicit differential innate immune responses following intramammary infection. Clin. Diagn. Lab. Immunol. 11:463472.

Bao, B., A. S. Prasad, F. W. Beck, G. W. Bao, T. Singh, S. Ali, and F. H. Sarkar. 2011. Intracellular free zinc up-regulates IFN- $\gamma$ and T-bet essential for Th1 differentiation in Con-A stimulated HUT-78 cells. Biochem. Biophys. Res. Commun. 407:703-707.

Bao, B., A. S. Prasad, F. W. Beck, and M. Godmere. 2003. Zinc modulates mRNA levels of cytokines. Am. J. Physiol. Endocrinol. Metab. 285:E1095-1102.

Barkema, H. W., Y. H. Schukken, and R. N. Zadoks. 2006. Invited Review: The role of cow, pathogen, and treatment regimen in the therapeutic success of bovine Staphylococcus aureus mastitis. J. Dairy Sci. 89:1877-1895.

Bauman, D. E. 1992. Bovine somatotropin: review of an emerging animal technology. J. Dairy Sci. 75:3432-3451.

Bauman, D. E. and W. B. Currie. 1980. Partitioning of nutrients during pregnancy and lactation: a review of mechanisms involving homeostasis and homeorhesis. J. Dairy Sci. 63:1514-1529.

Bell, A. W. 1995. Regulation of organic nutrient metabolism during transition from late pregnancy to early lactation. J. Anim. Sci. 73:2804-2819. 
Bell, A. W. and D. E. Bauman. 1997. Adaptations of glucose metabolism during pregnancy and lactation. J. Mammary Gland Biol. Neoplasia 2:265-278.

Bendich, A. 1993. Physiological role of antioxidants in the immune system. J. Dairy Sci. 76:2789-2794.

Bernabucci, U., B. Ronchi, N. Lacetera, and A. Nardone. 2005. Influence of body condition score on relationships between metabolic status and oxidative stress in periparturient dairy cows. J. Dairy Sci. 88:2017-2026.

Bjerknes, R., and D. Aarskog. 1995. Priming of human polymorphonuclear neutrophilic leukocytes by insulin-like growth factor I: increased phagocytic capacity, complement receptor expression, degranulation, and oxidative burst. J. Clin. Endocrinol. Metab. 80:1948-1955.

Böhmer, R.H., L. S. Trinkle and J. L. Staneck. 1992. Dose effects of LPS on neutrophils in a whole blood flow cytometric assay of phagocytosis and oxidative burst. Cytometry 13:525-531.

Borregaard, N., and T. Herlin. 1982. Energy metabolism of human neutrophils during phagocytosis. J. Clin. Invest. 70: 550-557.

Boyne, R., and J. R. Arthur. 1981. Effects of selenium and copper deficiency on neutrophil function in cattle. J. Comp. Pathol. 91:271-276.

Brigelius-Flohé, R., and L. Flohé. 2011. Basic principles and emerging concepts in the redox control of transcription factors. Antioxid. Redox Signal. 15:2335-2381.

Brinkmann, V., U. Reichard, C. Goosmann, B. Fauler, Y. Uhlemann, D. S. Weiss, Y. Weinrauch, and A. Zychlinsky. 2004. Neutrophil extracellular traps kill bacteria. Science 303:1532-1535.

Brinkmann, V., and A. Zychlinsky. 2007. Beneficial suicide: why neutrophils die to make NET. Nat. Rev. Microbiol. 5:577-82.Freitas, M., G. Porto, J. L. Lima, and E. Fernandes. 2009. Optimization of experimental settings for the analysis of human neutrophils oxidative burst in vitro. Talanta 78:1476-1483.

Brown, P. A., Davis W.C., Draghia-Akli R. 2004. Immune-enhancing effects of growth hormone-releasing hormone delivered by plasmid injection and electroporation. Mol. Ther. 10:644-651.

Burton, J. L., M. E. Kehrli Jr., S. Kapil, and R.L. Horst. 1995. Regulation of L-selectin and CD18 on bovine neutrophils by glucocorticoids: effects of cortisol and dexamethasone. J. Leuk. Biol. 57:317-325. 
Burton, J. L., and M. E. Kehrli Jr. 1996. Effects of dexamethasone on bovine circulating T-lymphocyte populations. J. Leuk. Biol. 59: 90-99.

Burton, J. L., S. A. Madsen, L. C. Chang, P. S. Weber, K. R. Buckham, R. van Dorp, M. C. Hickey, and B. Earley. 2005. Gene expression signatures in neutrophils exposed to glucocorticoids: a new paradigm to help explain "neutrophil dysfunction" in parturient dairy cows. Vet. Immunol. Immunopathol. 105:197219.

Burton, J. L., S. A. Madsen, J. Yao, S. S. Sipkovsky, and P. M. Coussens. 2001. An immunogenomics approach to understanding periparturient immunosuppression and mastitis susceptibility in dairy cows. Acta Vet. Scand. 42:407-424.

Burton, J. L., B. W. McBride, B. W. Kennedy, J. H. Burton, T. H. Elsasser, and B. Woodward. 1991. Influence of exogenous bovine somatotropin on the responsiveness of peripheral blood lymphocytes to mitogen. J. Dairy Sci. 74:916928.

Burton, J. L., B. W. McBride, B. W. Kennedy, J. H. Burton, T. H. Elsasser, and B. Woodward. 1992a. Contact sensitivity and systemic antibody responses in dairy cows treated with recombinant bovine somatotropin. J. Dairy Sci. 75:747-755.

Burton, J. L., B. W. McBride, B. W. Kennedy, J. H. Burton, T. H. Elsasser, and B. Woodward. 1992b. Hematological profiles in dairy cows treated with recombinant bovine somatotropin. J. Anim. Sci. 70:1488-1495.

Burvenich, C., D. D. Bannerman, J. D. Lippolis, L. Peelman, B. J. Nonnecke, M. E. Kehrli, Jr., and M. J. Paape. 2007. Cumulative physiological events influence the inflammatory response of the bovine udder to Escherichia coli infections during the transition period. J. Dairy Sci. 90 Suppl. 1:E39-54.

Burvenich, C., M. J. Paape, D. Hoeben, H. Dosogne, A. M. Massart-Leën, and J. Blum. 1999. Modulation of the inflammatory reaction and neutrophil defense of the bovine lactating mammary gland by growth hormone. Domest. Anim. Endocrinol. 17:149-159.

Burvenich, C., V. Van Merris, J. Mehrzad, A. Diez-Fraile, and L. Duchateau. 2003. Severity of E. coli mastitis is mainly determined by cow factors. Vet. Res. 34:521-564.

Cai, T. Q., P. G. Weston, L. A. Lund, B. Brodie, D. J. McKenna, and W. C. Wagner. 1994. Association between neutrophil functions and periparturient disorders in cows. Am. J. Vet. Res. 55:934-943.

Calder, P. C. 2008. The relationship between the fatty acid composition of immune cells and their function. Prostaglandins Leukot. Essent. Fatty Acids. 79:101-108. 
Cao, X. 2009. New DNA-sensing pathway feeds RIG-I with RNA. Nat. Immunol. 10:1049-1051.

Capuco, A. V., M. J. Paape, and S. C. Nickerson. 1986. In vitro study of polymorphonuclear leukocyte damage to mammary tissues of lactating cows. Am. J. Vet. Res. 47:663-668.

Cerone S. I., A. S. Sansinanea, S. A. Streitenberger, M. C. Garcia, and N. J. Auza. 2000. Cytochrome c oxidase, $\mathrm{Cu}, \mathrm{Zn}$-superoxide dismutase, and ceruloplasmin activities in copper-deficient bovines. Biol. Trace Elem. Res. 73:269-278.

Cha, E., D. Bar, J. A. Hertl, L. W. Tauer, G. Bennett, R. N. González, Y. H. Schukken, F. L. Welcome, and Y. T. Gröhn. 2011. The cost and management of different types of clinical mastitis in dairy cows estimated by dynamic programming. J. Dairy Sci. 94:4476-4487.

Chen, B., C. Wang, Y. M. Wang, and J. X. Liu. 2011. Effect of biotin on milk performance of dairy cattle: a meta-analysis. J. Dairy Sci. 94:3537-3546.

Chow J. C., D. W. Young, D. T. Golenbock, W. J. Christ, and F. Gusovsky. 1999. Tolllike receptor-4 mediates lipopolysaccharide-induced signal transduction. J. Biol. Chem. 274:10689-10692.

Clark, R. 1997. The somatogenic hormones and insulin-like growth factor-1: stimulators of lymphopoiesis and immune function. Endocr. Rev. 18:157-179.

Collings, L. K., D. M. Weary, N. Chapinal, and M. A. von Keyserlingk. 2011. Temporal feed restriction and overstocking increase competition for feed by dairy cattle. J. Dairy Sci. 94:5480-5486.

Contreras, G. A., N. J. O'Boyle, T. H. Herdt, and L. M. Sordillo. 2010. Lipomobilization in periparturient dairy cows influences the composition of plasma nonesterified fatty acids and leukocyte phospholipid fatty acids. J. Dairy Sci. 93:2508-2516.

Cullum, R., O. Alder, and P. A. Hoodless. 2011. The next generation: using new sequencing technologies to analyse gene regulation. Respirology 16:210-222.

Dahl, G. E. 2008. Effects of short day photoperiod on prolactin signaling in dry cows: a common mechanism among tissues and environments? J. Anim. Sci. 86:10-14.

Daniel, L. R., B. P. Chew, T. S. Tanaka, and L. W. Tjoelker. 1991. Beta-carotene and vitamin A effects on bovine phagocyte function in vitro during the peripartum period. J. Dairy Sci. 74:124-131. 
Dann, H. M., N. B. Litherland, J. P. Underwood, M. Bionaz, A. D'Angelo, J. W. McFadden, J. K. Drackley. 2006. Diets during far-off and close-up dry periods affect periparturient metabolism and lactation in multiparous cows. J. Dairy Sci. 89:3563-3577.

DAVID Bioinformatics, 2012. Accessed Mar 2012. http://david.abcc.ncifcrf.gov.

Davis, A. R., P. L. Mascolo, P. L. Bunger, K. M. Sipes, and M. T. Quinn. 1998. Cloning and sequencing of the bovine flavocytochrome $\mathrm{b}$ subunit proteins, gp91-phox and p22-phox: comparison with other known flavocytochrome $b$ sequences. J. Leukoc. Biol. 64:114-123.

De Schepper, S., A. De Ketelaere, D. D. Bannerman DD, M. J. Paape, L. Peelman and C. Burvenich. 2008. The toll-like receptor-4 (TLR-4) pathway and its possible role in the pathogenesis of Escherichia coli mastitis in dairy cattle. Vet. Res. 39:05-28.

Detilleux, J. C. 2004. Neutrophils in the war against Staphylococcus aureus: Predatorprey models to the rescue. J. Dairy Sci. 87:3716-3724.

Detilleux, J. C., M. E. Kehrli Jr., J. R. Stabel, A. E. Freeman, and D. H. Kelley. 1995. Study of immunological dysfunction in periparturient Holstein cattle selected for high and average milk production. Vet. Immunol. Immunopathol. 44:251-267.

Detilleux, J. C., K. J. Koehler, A. E. Freeman, M. E. Jr. Kehrli, and D. H. Kelley. 1994. Immunological parameters of periparturient Holstein cattle: genetic variation. J. Dairy Sci. 77:2640-2650.

Diez-Fraile, A., E. Meyer, and C. Burvenich. 2002. Regulation of adhesion molecules on circulating neutrophils during coliform mastitis and their possible immunomodulation with drugs. Vet. Immunol. Immunopathol. 86:01-10.

Dinauer, M.C., E. A. Pierce, R. W. Erickson, T. J. Muhlebach, H. Messner, S. H. Orkin, R. A. Seger, and J. T. Curnutte. 1991. Point mutation in the cytoplasmic domain of the neutrophil p22-phox cytochrome $b$ subunit is associated with a nonfunctional NADPH oxidase and chronic granulomatous disease. Proc. Natl. Acad. Sci. U. S. A. 88:11231-11235.

Drackley, J. K. 1999. Biology of dairy cows during the transition period: the final frontier. J. Dairy Sci. 82:2259-2273.

Drackley, J. K., T. R. Overton, and G. N. Douglas. 2001. Adaptations of glucose and long chain fatty acid metabolism in liver of dairy cows during the periparturient period. J. Dairy Sci. 84:E100-E112.

Dorshkind, K., and N. D. Horseman. Anterior pituitary hormones, stress, and immune system homeostasis. 2001. Bioessays 23:288-294. 
Dosogne, H., C. Burvenich, A. E. Freeman, M. E. Kehrli, Jr., J. C. Detilleux, J. Sulon, J. F. Beckers, and D. Hoeben. 1999. Pregnancy-associated glycoprotein and decreased polymorphonuclear leukocyte function in early post-partum dairy cows. Vet. Immunol. Immunopathol. 67:47-54.

Dosogne, H., F. Vangroenweghe, B. Barrio, P. Rainard, and C. Burvenich. 2001. Decreased number and bactericidal activity against Staphylococcus aureus of the resident cells in milk of dairy cows during early lactation. J. Dairy Res. 68:539549.

Duthie, S. J., and A. Hawdon. 1998. DNA instability (strand breakage, uracil misincorporation, and defective repair) is increased by folic acid depletion in human lymphocytes in vitro. FASEB J. 12:1491-1497.

Elsik, C. G., R. L. Tellam, K. C. Worley, R. A. Gibbs, D. M. Muzny, et al. 2009. The genome sequence of taurine cattle: a window to ruminant biology and evolution. Science 324: 522-528.

Elvinger, F., P. J. Hansen, H. H. Head, and R. P. Natzke. 1991. Actions of bovine somatotropin on polymorphonuclear leukocytes and lymphocytes in cattle. J. Dairy Sci. 74:2145-2152.

Eskelinen, E. L., Y. Tanaka, and P. Saftig. 2003. At the acidic edge: emerging functions for lysosomal membrane proteins. Trends Cell Biol. 13:137-145.

Forehand, J.R., M. J. Pabst, W. A. Phillips, and R.B. Johnston Jr. 1989. Lipopolysaccharide priming of human neutrophils for an enhanced respiratory burst. Role of intracellular free calcium. J. Clin. Invest. 83:74-83.

Formigoni, A., M. Fustini, L. Archetti, S. Emanuele, C. Sniffen, and G. Biagi. 2011. Effects of an organic source of copper, manganese and zinc on dairy cattle productive performance, health status and fertility. Anim. Feed Sci. Technol. 164:191-198.

Freitas, M., G. Porto, J. L. Lima, and E. Fernandes. 2009. Optimization of experimental settings for the analysis of human neutrophils oxidative burst in vitro. Talanta 78:1476-1483.

Fukai, T., and M. Ushio-Fukai. 2011. Superoxide dismutases: role in redox signaling, vascular function, and diseases. Antioxid. Redox Signal. 15:1583-1606.

Goff, J. P. 2006. Major advances in our understanding of nutritional influences on bovine health. J. Dairy Sci. 89:1292-1301. 
Goff, J. P., and R. L. Horst. 1997. Physiological changes at parturition and their relationship to metabolic disorders. J. Dairy Sci. 80:1260-1268.

Graulet, B., J. J. Matte, A. Desrochers, L. Doepel, M. F. Palin, and C. L. Girard. 2007. Effects of dietary supplements of folic acid and vitamin B12 on metabolism of dairy cows in early lactation. J. Dairy Sci. 90:3442-3455.

Grimble, R. F. 1997. Effect of antioxidative vitamins on immune function with clinical applications. Int. J. Vitam. Nutr. Res. 67:312-320.

Grinberg, N., Elazar S., Rosenshine I. and Shpigel N.Y. 2008. Beta-hydroxybutyrate abrogates formation of bovine neutrophil extracellular traps and bactericidal activity against mammary pathogenic Escherichia coli. Infect. Immun. 76:28022807.

Grummer, R. R. 1993. Etiology of lipid-related metabolic disorders in periparturient dairy cows. J. Dairy Sci. 76:3882-3896.

Gry, M., R. Rimini, S. Strömberg, A. Asplund, F. Pontén, M. Uhlén, and P. Nilsson. 2009. Correlations between RNA and protein expression profiles in 23 human cell lines. BMC Genomics 10:365-379.

Guidry, A. J., L. M. Berning, and C. N. Hambleton. 1993. Opsonization of Staphylococcus aureus by bovine immunoglobulin isotypes. J. Dairy Sci. 76:1285-1289.

Guidry, A. J., M. J. Paape, and R. E. Pearson. 1975. Effects of estrus and exogenous estrogen on circulating neutrophils and milk somatic cell concentration, neutrophil phagocytosis, and occurrence of clinical mastitis in cows. Am. J. Vet. Res. 36:1555-1560.

Guidry, A. J., M. J. Paape, and R. E. Pearson. 1976. Effects of parturition and lactation on blood and milk cell concentrations, corticosteroids, and neutrophil phagocytosis in the cow. Am. J. Vet. Res. 37:1195-1200.

Haase H., and L. Rink. 2009. Functional significance of zinc-related signaling pathways in immune cells. Annu. Rev. Nutr. 29:133-152.

Hackbart, K. S., R. M. Ferreira, A. A. Dietsche, M. T. Socha, R. D. Shaver, M. C. Wiltbank, and P. M. Fricke. 2010. Effect of dietary organic zinc, manganese, copper, and cobalt supplementation on milk production, follicular growth, embryo quality, and tissue mineral concentrations in dairy cows. J. Anim. Sci. 88:38563870 . 
Hakogi, E., H. Tamura, S. Tanaka, A. Kohata, Y. Shimada and K. Tabuchi. 1989. Endotoxin levels in milk and plasma of mastitis-affected cows measured with a chromogenic limulus test. Vet. Microbiol. 20:267-274

Hammon, D. S., I. M. Evjen, T. R. Dhiman, J. P. Goff, and J. L. Walters. 2006. Neutrophil function and energy status in Holstein cows with uterine health disorders. Vet. Immunol. Immunopathol. 113:21-29.

Harmon, R. J. 1994. Physiology of mastitis and factors affecting somatic cell counts. J. Dairy Sci. 77:2103-2112.

Haslett, C., L. A. Guthrie, M. M. Kopaniak, R. B. Johnston Jr., and P. M. Henson. 1985. Modulation of multiple neutrophil functions by preparative methods or trace concentrations of bacterial lipopolysaccharide. Am. J. Pathol. 119:101-110.

Hayashi, F., T. K. Means, and A. D. Luster. 2003. Toll-like receptors stimulate human neutrophil function. Blood 102:2660-2669.

Hayirli, A., D. H. Keisler, L. Doepel, and H. Petit. 2011. Peripartum responses of dairy cows to prepartal feeding level and dietary fatty acid source. J Dairy Sci. 94:917930.

Hensen, S. M., M. J. Pavicić, J. A. Lohuis, J. A. de Hoog, and B. Poutrel. 2000. Location of Staphylococcus aureus within the experimentally infected bovine udder and the expression of capsular polysaccharide type 5 in situ. J. Dairy Sci. 83:1966-1975.

Heyneman, R., C. Burvenich, and R. Vercauteren. 1990. Interaction between the respiratory burst activity of neutrophil leukocytes and experimentally induced $E$. coli mastitis in cows. J. Dairy Sci. 73:985-994.

Hock, H., M. J. Hamblen, H. M. Rooke, D. Traver, R. T. Bronson, S. Cameron, and S. H. Orkin. 2003. Intrinsic requirement for zinc finger transcription factor Gfi-1 in neutrophil differentiation. Immunity 18:109-120.

Hodgson, J. C., C. A. Watkins, and C. W. Bayne. 2006. Contribution of respiratory burst activity to innate immune function and the effects of disease status and agent on chemiluminescence responses by ruminant phagocytes in vitro. Vet. Immunol. Immunopathol. 112:12-23.

Hoeben, D., C. Burvenich, and A. M. Massart-Leen. 1998. Glucocorticosteroids and in vitro effects on chemiluminescence of isolated bovine blood granulocytes. Eur. J. Pharmacol. 354:197-203.

Hoeben, D., C. Burvenich, P. J. Eppard, and D. L. Hard. 1999a. Effect of recombinant bovine somatotropin on milk production and composition of cows with Streptococcus uberis mastitis. J. Dairy Sci. 82:1671-1683. 
Hoeben, D., C. Burvenich, P. J. Eppard, J. C. Byatt, and D. L. Hard. 1999b. Effect of bovine somatotropin on neutrophil functions and clinical symptoms during Streptococcus uberis mastitis. J. Dairy Sci. 82:1465-1481.

Hoeben, D., R. Heyneman, and C. Burvenich. 1997. Elevated levels of betahydroxybutyric acid in periparturient cows and in vitro effect on respiratory burst activity of bovine neutrophils. Vet. Immunol. Immunopathol. 58:165-170.

Hoeben, D., E. Monfardini, G. Opsomer, C. Burvenich, H. Dosogne, A. De Kruif, and J. F. Beckers. 2000. Chemiluminescence of bovine polymorphonuclear leucocytes during the periparturient period and relation with metabolic markers and bovine pregnancyassociated glycoprotein. J. Dairy Res. 67:249-259.

Hogan, J. S., and K. L. Smith. 2003. Coliform mastitis. Vet. Res. 34:507-519.

Hogeveen, H., K. Huijps, and T. J. Lam. 2011. Economic aspects of mastitis: new developments. N. Z. Vet. J. 59:16-23.

Holley, A.K., S. K. Dhar, and D. K. St Clair. 2010. Manganese superoxide dismutase versus p53: the mitochondrial center. Ann. N. Y. Acad. Sci. 1201:72-78.

Horst R. L., J. P. Goff, T. A. Reinhardt, and D. R. Buxton. 1997. Strategies for preventing milk fever in dairy cattle. J. Dairy Sci. 80:1269-1280.

Hensen, S. M., M. J. Pavicić, J. A. Lohuis, J. A. de Hoog, and B. Poutrel. 2000. Location of Staphylococcus aureus within the experimentally infected bovine udder and the expression of capsular polysaccharide type 5 in situ. J. Dairy Sci. 83:1966-1975.

Huang, D. W., B. T. Sherman, R. A. Lempicki. 2009a. Bioinformatics enrichment tools: paths toward the comprehensive functional analysis of large gene lists. Nucleic Acids Res. 37:1-13.

Huang, D. W., B. T. Sherman, R. A. Lempicki. 2009b. Systematic and integrative analysis of large gene lists using DAVID Bioinformatics Resources. Nature Protoc. 4:44-57.

Ibeagha-Awemu, E. M., J. W. Lee, A. E. Ibeagha, D. D. Bannerman, M. J. Paape, and X. Zhao. 2008. Bacterial lipopolysaccharide induces increased expression of toll-like receptor (TLR) 4 and downstream TLR signaling molecules in bovine mammary epithelial cells. Vet. Res. 39:11-23.

Ishikawa, H., T. Shirahata, and K. Hasegawa. 1994. Interferon-gamma production of mitogen stimulated peripheral lymphocytes in perinatal cows. J. Vet. Med. Sci. 56:735-738. 
Justice-Allen, A., J. Trujillo, R. Corbett, R. Harding, G. Goodell, and D. Wilson. 2010. Survival and replication of Mycoplasma species in recycled bedding sand and association with mastitis on dairy farms in Utah. J. Dairy Sci. 93:192-202.

Kahl, S., T. H. Elsasser, and C. J. Li. 2011. Modeling the effects of estradiol and progesterone on the acute phase proinflammatory axis: variability in tumor necrosis factor- $\alpha$, nitric oxide, and xanthine oxidase responses to endotoxin challenge in steers. Domest. Anim. Endocrinol. 40:213-221.

Kaps, M., and W. Lamberson. 2004. Biostatistics for Animal Science. Wallingford: CAB International, Cambridge, MA.

Kauf A.C., B. T. Vinyard, and D. D. Bannerman. 2007. Effect of intramammary infusion of bacterial lipopolysaccharide on experimentally induced Staphylococcus aureus intramammary infection. Res. Vet. Sci. 82:39-46.

Kawai, T., and Akira S. 2008. Toll-like receptor and RIG-I-like receptor signaling. Ann. N. Y. Acad. Sci. 1143:1-20.

Kehrli, M. E. Jr, and J. P. Goff. 1989. Periparturient hypocalcemia in cows: effects on peripheral blood neutrophil and lymphocyte function. J. Dairy Sci. 72:1188-1196.

Kehrli, M. E. Jr., B. J. Nonnecke, and J. A. Roth. 1989. Alterations in bovine lymphocyte function during the periparturient period. Am. J. Vet. Res. 50:215-220.

Kellogg, D. W., D. J. Tomlinson, M. T. Socha, and A. B. Johnson. 2004. Effect of feeding zinc methionine complex on milk production and somatic cell count of dairy cattle: Twelve-trial summary. Prof. Anim. Sci. 20:295-301.

Kimura, K., J. P. Goff, and M. E. Kehrli, Jr. 1999. Effects of the presence of the mammary gland on expression of neutrophil adhesion molecules and myeloperoxidase activity in periparturient dairy cows. J. Dairy Sci. 82:2385-2392.

Kimura, K., J. P. Goff, M. E. Kehrli Jr., J. A. Harp, and B. J. Nonnecke. 2002a. Effects of mastectomy on composition of peripheral blood mononuclear cell populations in periparturient dairy cows. J. Dairy Sci. 85:1437-1444.

Kimura, K., J. P. Goff, M. E. Kehrli Jr, and T. A. Reinhardt. 2002b. Decreased neutrophil function as a cause of retained placenta in dairy cattle. J. Dairy Sci. 85:544-50.

Kimura, K., Reinhardt T.A. and Goff J.P. 2006. Parturition and hypocalcemia blunts calcium signals in immune cells of dairy cattle. J. Dairy Sci. 89:2588-2595.

Kincaid, R. L., B. P. Chew, and J. D. Cronrath. 1997. Zinc oxide and amino acids as sources of dietary zinc for calves: effects on uptake and immunity. J. Dairy Sci. 80:1381-1388. 
KEGG (Kyoto Encyclopedia of Genes and Genomes), 2012. Accessed Mar. 2012. http://www.genome.jp/kegg.

Kwong, L.S., R. Parsons, R. Patterson, T. J. Coffey, L. Thonur, J. S. Chang, G. Russell, D. Haig, D. Werling, and J. C. Hope. 2011. Characterisation of antibodies to bovine Toll-like receptor (TLR)-2 and cross-reactivity with ovine TLR2. Vet. Immunol. Immunopathol. 139:313-318.

Kwak, H. K., C. M. Hansen, J. E. Leklem, K. Hardin, and T. D. Shultz. 2002. Improved vitamin B-6 status is positively related to lymphocyte proliferation in young women consuming a controlled diet. J. Nutr. 132:3308-3313.

Lacetera, N., D. Scalia, U. Bernabucci, B. Ronchi, D. Pirazzi, and A. Nardone. 2005. Lymphocyte functions in overconditioned cows around parturition. J. Dairy Sci. 88:2010-2016.

Lamkanfi, M., and V. M. Dixit. 2011. Modulation of inflammasome pathways by bacterial and viral pathogens. J. Immunol. 187:597-602.

Lamote, I., E. Meyer, A. De Ketelaere, L. Duchateau, and C. Burvenich. 2006. Expression of the estrogen receptor in blood neutrophils of dairy cows during the periparturient period. Theriogenology 65:1082-1098.

Laudanna, C., G. Constantin, P. Baron, E. Scarpini, G. Scarlato, G. Cabrini, C. Dechecchi, F. Rossi, M. A. Cassatella, and G. Berton. 1994. Sulfatides trigger increase of cytosolic free calcium and enhanced expression of tumor necrosis factor-alpha and interleukin- 8 mRNA in human neutrophils. Evidence for a role of L-selectin as a signaling molecule. J. Biol. Chem. 269:4021-4026.

Lauzon K., X. Zhao, A. Bouetard, L. Delbecchi, B. Paquette, and P. Lacasse. 2005. Antioxidants to prevent bovine neutrophil-induced mammary epithelial cell damage. J. Dairy Sci. 88:4295-4303.

LeBlanc, S. J., K. D. Lissemore, D. F. Kelton, T. F. Duffield, and K. E. Leslie. 2006. Major advances in disease prevention in dairy cattle. J. Dairy Sci. 89:1267-1279.

Lee, W. L., R. E. Harrison, and S. Grinstein. 2003. Phagocytosis by neutrophils. Microbes Infect. 5:1299-1306.

Lee, E. K., and M. E. Kehrli Jr. 1998. Expression of adhesion molecules on neutrophils of periparturient cows and neonatal calves. Am. J. Vet. Res. 59:37-43.

Lehtolainen, T., S. Suominen, T. Kutila, and S. Pyörälä. 2003. Effect of intramammary Escherichia coli endotoxin in early- vs. late-lactating dairy cows. J. Dairy Sci. 86:2327-2333. 
Lessard, M., N. Gagnon, and H. V. Petit. 2003. Immune response of postpartum dairy cows fed flaxseed. J. Dairy Sci. 86:2647-2657.

Lippolis, J. D. 2008. Immunological signaling networks: integrating the body's immune response. J. Anim. Sci. 86:E53-63.

Lippolis, J. D., B. D. Peterson-Burch, and T. A. Reinhardt. 2006a. Differential expression analysis of proteins from neutrophils in the periparturient period and neutrophils from dexamethasone-treated dairy cows. Vet. Immunol. Immunopathol. 111:149164.

Lippolis, J. D., T. A. Reinhardt, J. P. Goff, and R. L. Horst. 2006b. Neutrophil extracellular trap formation by bovine neutrophils is not inhibited by milk. Vet. Immunol. Immunopathol. 113:248-255.

Littell, R. C., P. R. Henry, and C. B. Ammerman. 1998. Statistical analysis of repeated measures data using SAS procedures. J. Anim. Sci. 76:1216-1231.

Livak, K.J. and T. D. Schmittgen. 2001. Analysis of relative gene expression data using real-time quantitative PCR and the 2(-Delta Delta C(T)) Method. Methods 25:402-408.

Lögters, T., S. Margraf, J. Altrichter, J. Cinatl, S. Mitzner, J. Windolf, and M. Scholz. 2009. The clinical value of neutrophil extracellular traps. Med. Microbiol. Immunol. 198:211-219.

MacPherson, A., D. Gray, G. B. Mitchell, and C. N. Taylor. 1987. Ostertagia infection and neutrophil function in cobalt-deficient and cobalt-supplemented cattle. $\mathrm{Br}$. Vet. J. 143:348-353.

Madsen, S. A., L. C. Chang, M. C. Hickey, G. J. Rosa, P. M. Coussens, and J. L. Burton. 2004. Microarray analysis of gene expression in blood neutrophils of parturient cows. Physiol. Genomics 16:212-221.

Madsen, S. A., P. S. Weber, and J. L. Burton. 2002. Altered expression of cellular genes in neutrophils of periparturient dairy cows. Vet. Immunol. Immunopathol. 86(34):159-175

Mallard, B. A., J. C. Dekkers, M. J. Ireland, K. E. Leslie, S. Sharif, C. L. Vankampen, L. Wagter, and B. N. Wilkie. 1998. Alteration in immune responsiveness during the peripartum period and its ramification on dairy cow and calf health. J. Dairy Sci. 81:585-595. 
Maratou, E., G. Dimitriadis, A. Kollias, E. Boutati, V. Lambadiari, P. Mitrou, and S. A. Raptis. 2007. Glucose transporter expression on the plasma membrane of resting and activated white blood cells. Eur. J. Clin. Invest. 37:282-290.

Mashek, D. G., S. J. Bertics, and R. R. Grummer. 2005. Effects of intravenous triacylglycerol emulsions on hepatic metabolism and blood metabolites in fasted dairy cows. J. Dairy Sci. 88:100-109

McCarthy, D. J., Y. Chen Y., and G. K. Smyth. 2012. Differential expression analysis of multifactor RNA-Seq experiments with respect to biological variation. Nucleic Acids Res. 40:4288-4297.

McClenahan, D., J. Fagliari, O. Evanson, and D. Weiss. 2000. Role of inflammatory mediators in priming, activation, and deformability of bovine neutrophils. Am. J. Vet. Res. 61:492-498.

Mehrzad, J., H. Dosogne, E. Meyer, and C. Burvenich. 2001a. Local and systemic effects of endotoxin mastitis on the chemiluminescence of milk and blood neutrophils in dairy cows. Vet. Res. 32:131-144.

Mehrzad, J., H. Dosogne, E. Meyer, R. Heyneman, and C. Burvenich. 2001b. Respiratory burst activity of blood and milk neutrophils in dairy cows during different stages of lactation. J. Dairy Res. 68:399-415.

Mehrzad, J., L. Duchateau, S. Pyorala, and C. Burvenich. 2002. Blood and milk neutrophil chemiluminescence and viability in primiparous and pluriparous dairy cows during late pregnancy, around parturition and early lactation. J. Dairy Sci. $85: 3268-3276$.

Menegazzi, R., E. Decleva, and P. Dri. 2011. Killing by neutrophil extracellular traps: fact or folklore? Blood 119:1214-1216.

Miller, W. J. 1975. New concepts and developments in metabolism and homeostasis of inorganic elements in dairy cattle. A review. J. Dairy Sci. 58:1549-1560.

Miller, J. K., E. Brzezinska-Slebodzinska, and F. C. Madsen. 1993. Oxidative stress, antioxidants, and animal function. J. Dairy Sci. 76:2812-2823.

Moore, J. B., R. K. Blanchard, W. T. McCormack, and R. J. Cousins. 2001. cDNA array analysis identifies thymic LCK as upregulated in moderate murine zinc deficiency before T-lymphocyte population changes. J. Nutr. 131:3189-3196.

Moroni, F., M. L. Di Paolo, A. Rigo, C. Cipriano, R. Giacconi, R. Recchioni, F. Marcheselli, M. Malavolta, and E. Mocchegiani. 2005. Interrelationship among neutrophil efficiency, inflammation, antioxidant activity and zinc pool in very old age. Biogerontology 6:271-281. 
Mortazavi, A., B. A. Williams, K. McCue, L. Schaeffer, and B. Wold. 2008. Mapping and quantifying mammalian transcriptomes by RNA-Seq. Nat. Methods 5:621628.

Moyes, K. M., J. K. Drackley, D. E. Morin, and J. J. Loor. 2010. Greater expression of TLR2, TLR4, and IL6 due to negative energy balance is associated with lower expression of HLA-DRA and HLA-A in bovine blood neutrophils after intramammary mastitis challenge with Streptococcus uberis. Funct. Integr. Genomics 10:53-61.

Moyes, K. M., J. K. Drackley, J. L. Salak-Johnson, D. E. Morin, J. C. Hope, and J. J. Loor. 2009. Dietary-induced negative energy balance has minimal effects on innate immunity during a Streptococcus uberis mastitis challenge in dairy cows during midlactation. J. Dairy Sci. 92:4301-4316.

Mullarky, I. K., C. Su, N. Frieze, Y. H. Park, and L. M. Sordillo. 2001. Staphylococcus aureus agr genotypes with enterotoxin production capabilities can resist neutrophil bactericidal activity. Infect. Immun. 69:45-51.

Murakami M., and T. Hirano. 2008. Intracellular zinc homeostasis and zinc signaling. Cancer Sci. 99:1515-1522.

Myllys, V., T. Honkanen-Buzalski, H. Virtanen, S. Pyörälä, and H. P. Müller. 1994. Effect of abrasion of teat orifice epithelium on development of bovine staphylococcal mastitis. Dairy Sci. 77:446-452.

Nagahata, H., H. Higuchi, O. Inanami, and M. Kuwabara. 2007. Costimulatory effects of complement receptor type 3 and Fc receptor for IgG (FcgammaR) on superoxide production and signal transduction in bovine neutrophils. J. Vet. Med. Sci. 69:993-997.

National Center for Biotechnology Information. 2010. Accessed Oct. 2010. http://blast.ncbi.nlm.nih.gov/.

National Research Council. 2001. Nutrient Requirements of Dairy Cattle. 7th rev. ed. National Academy Press, Washington, DC.

Neefjes, J., M. L. Jongsma, P. Paul, and O. Bakke. 2011. Towards a systems understanding of MHC class I and MHC class II antigen presentation. Nat. Rev. Immunol. 11:823-836.

Newburger, P. E., Y. V. Subrahmanyam, and S. M. Weissman. 2000. Global analysis of neutrophil gene expression. Curr. Opin. Hematol. 7:16-20. 
Nielsen, L., C. M. Røntved, M. O. Nielsen, L. R. Norup, and K. L. Ingvartsen. 2003. Leukocytes from heifers at different ages express insulin and insulin-like growth factor-1 (IGF-1) receptors. Domest. Anim. Endocrinol. 25:231-238.

Nishimura, H., A. Gogami, Y. Miyagawa, A. Nanbo, Y. Murakami, T. Baba, and S. Nagasawa. 2001. Bactericidal/permeability-increasing protein promotes complement activation for neutrophil-mediated phagocytosis on bacterial surface. Immunology 103:519-525.

NMC. 1996. Current concepts in bovine mastitis. National Mastitis Council, Madison, WI.

Nocek, J. E., M. T. Socha, and D. J. Tomlinson. 2006. The effect of trace mineral fortification level and source on performance of dairy cattle. J. Dairy Sci. 89:2679-2693.

Nonnecke, B. J., J. L. Burton, and M. E. Kehrli Jr. 1997. Associations between function and composition of blood mononuclear leukocyte populations from Holstein bulls treated with dexamethasone. J. Dairy Sci. 80:2403-2410.

Nonnecke, B. J., S. T. Franklin, and Y. W. Young. 1992. Effects of ketones, acetate, and glucose on in vitro immunoglobulin secretion by bovine lymphocytes. J. Dairy Sci. 75:982-990.

Nonnecke, B. J., and J. A. Harp. 1989. Function and regulation of lymphocyte-mediated immune responses: relevance to bovine mastitis. J. Dairy Sci. 72:1313-1327.

Nonnecke, B. J., K. Kimura, J. P. Goff, and M. E. Kehrli Jr. 2003. Effects of the mammary gland on functional capacities of blood mononuclear leukocyte populations from periparturient cows. J. Dairy Sci. 86:2359-2368.

Nordenfelt, P., and H. Tapper. 2011. Phagosome dynamics during phagocytosis by neutrophils. J. Leukoc. Biol. 90:271-284.

Opdebeeck, J. P., A. J. Frost, and D. O'Boyle. 1988. Adhesion of Staphylococcus aureus and Escherichia coli to bovine udder epithelial cells. Vet. Microbiol. 16:77-86.

Ohtsuka, H., Kase H., Ando T., Kohiruimaki M., Masui M., Watanabe D., Hayashi T. and Kawamura S. 2008. Changes in peripheral blood mononuclear cells after calving in lactating cows with serious decrease of body weight before and after calving. J. Vet. Med. Sci. 70:153-158.

Oliver, S. P., and L. M. Sordillo. 1988. Udder health in the periparturient period. J. Dairy Sci. 71:2584-2606. 
Olsson, J., T. A. Jacobson, J. M. Paulsson, E. Dadfar, A. Moshfegh, S. H. Jacobson, and J. Lundahl. 2010. Expression of neutrophil SOD2 is reduced after lipopolysaccharide stimulation: a potential cause of neutrophil dysfunction in chronic kidney disease. Nephrol. Dial. Transplant. 26:2195-2201.

Overton, T. R. 1998. Substrate utilization for hepatic gluconeogenesis in the transition dairy cow. Pages 237-246 in Cornell Nutr. Conf. for Feed Manufacturers. Cornell Univ., Ithaca, NY.

Overton, T. R. and M. R. Waldron. 2004. Nutritional Management of Transition Dairy Cows: Strategies to Optimize Metabolic Health. J. Dairy Sci. 87:E105-119.

Paape, M. J., D. D. Bannerman, X. Zhao, and J. W. Lee. 2003. The bovine neutrophil: Structure and function in blood and milk. Vet. Res. 34:597-627.

Paape, M. J., E. M. Lilius, P. A. Wiitanen, M. P. Kontio, and R. H. Miller. 1996. Intramammary defense against infections induced by Escherichia coli in cows. Am. J. Vet. Res. 57:477-482.

Paape, M. J., J. Mehrzad, X. Zhao, J. Detilleux, and C. Burvenich. 2002. Defense of the bovine mammary gland by polymorphonuclear neutrophil leukocytes. J. Mammary Gland Biol. Neoplasia. 7:109-121.

Paterson, J. E., and A. MacPherson. 1990. The influence of a low cobalt intake on the neutrophil function and severity of Ostertagia infection in cattle. Br. Vet. J. 146:519-530.

Papayannopoulos, V., and A. Zychlinsky. 2009. NET: a new strategy for using old weapons. Trends Immunol. 11:513-521.

Park, Y. H., L. K. Fox, M. J. Hamilton, and W. C. Davis. 1992. Bovine mononuclear leukocyte subpopulations in peripheral blood and mammary gland secretions during lactation. J. Dairy Sci. 75:998-1006.

Perkins, K. H., M. J. VandeHaar, R. J. Tempelman, and J. L. Burton. 2001. Negative energy balance does not decrease expression of leukocyte adhesion or antigenpresenting molecules in cattle. J. Dairy Sci. 84:421-428.

Perkins, K. H., M. J. VandeHaar, J. L. Burton, J. S. Liesman, R. J. Erskine, and T. H. Elsasser. 2002. Clinical responses to intramammary endotoxin infusion in dairy cows subjected to feed restriction. J. Dairy Sci. 85:1724-1731.

Persson, K., I. Larsson, and C. Hallén Sandgren. 1993. Effects of certain inflammatory mediators on bovine neutrophil migration in vivo and in vitro. Vet. Immunol. Immunopathol. 37:99-112. 
Petit, H. V., M. F. Palin, and L. Doepel. 2007. Hepatic lipid metabolism in transition dairy cows fed flaxseed. J. Dairy Sci. 90:4780-4792.

Piessens, V., E. Van Coillie, B. Verbist, K. Supré, G. Braem, A. Van Nuffel, L. De Vuyst, M. Heyndrickx, and S. De Vliegher. 2011. Distribution of coagulasenegative Staphylococcus species from milk and environment of dairy cows differs between herds. J. Dairy Sci. 94:2933-2944.

Prasad A.S. 2007. Zinc: mechanisms of host defense. J. Nutr. 137:1345-1349.

Predieri G., L. Elviri, M. Tegoni, I. Zagnoni, E. Cinti, G. Biagi, S. Ferruzza, and G. Leonardi. 2005. Metal chelates of 2-hydroxy-4-methylthiobutanoic acid in animal feeding. Part 2: Further characterizations, in vitro and in vivo investigations. J. Inorg. Biochem. 99:627-636.

Prinz, I. 2011. Dynamics of the interaction of $\gamma \delta$ T cells with their neighbors in vivo. Cell Mol. Life Sci. 68:2391-2398.

Remijsen, Q., T. Vanden Berghe, E. Wirawan, B. Asselbergh, E. Parthoens, R. De Rycke, S. Noppen, M. Delforge, J. Willems, and P. Vandenabeele. 2011. Neutrophil extracellular trap cell death requires both autophagy and superoxide generation. Cell Res. 21:290-304.

Revelo, X. S., and M. R. Waldron. 2010. Effects of in vitro insulin and 2,4thiazolidinedione on the function of neutrophils harvested from blood of cows in different physiological states. J. Dairy Sci. 93:3990-4005.

Revelo, X. S., and M. R. Waldron. 2012. In vitro effects of Escherichia coli lipopolysaccharide on the function and gene expression of neutrophils isolated from the blood of dairy cows. J. Dairy Sci. 95:2422-2441.

Rinaldi, M., P. Moroni, L. Leino, J. Laihia, M. J. Paape, and D. D. Bannerman. 2006. Effect of cis-urocanic acid on bovine neutrophil generation of reactive oxygen species. J. Dairy Sci. 89:4188-4201.

Rinaldi, M., P. Moroni, M. J. Paape, and D. D. Bannerman. 2007. Evaluation of assays for the measurement of bovine neutrophil reactive oxygen species. Vet. Immunol. Immunopathol. 115:107-125.

Riollet, C., P. Rainard, and B. Poutrel. 2000. Cells and cytokines in inflammatory secretions of bovine mammary gland. Adv. Exp. Med. Biol. 480:247-258.

Roberson, J. R., L. K. Fox, D. D. Hancock, J. M. Gay, and T. E. Besser. 1994. Ecology of Staphylococcus aureus isolated from various sites on dairy farms. J. Dairy Sci. 77:3354-3364. 
Robinson, M. D., D. J. McCarthy, and G. K. Smyth. 2010. edgeR: a bioconductor package for differential expression analysis of digital gene expression data. Bioinformatics 26:139-140.

Rowson, A. D., Y. Q. Wang, E. Aalseth, N. E. Forsberg, and S. B. Puntenney. 2011. Effects of an immunomodulatory feed additive on the development of mastitis in a mouse infection model using four bovine-origin isolates. Animal 5:220-229.

Saad, A. M., C. Concha, and G. Aström. 1989. Alterations in neutrophil phagocytosis and lymphocyte blastogenesis in dairy cows around parturition. J. Vet. Med. 36:337345.

Saad, A. M., and K. Ostensson. 1990. Flow cytofluorometric studies on the alteration of leukocyte populations in blood and milk during endotoxin-induced mastitis in cows. Am. J. Vet. Res. 51:1603-1607.

Sander, A. K., M. Piechotta, G. Schlamberger, H. Bollwein, M. Kaske, A. Sipka, and H. J. Schuberth. 2011. Ex vivo phagocytic overall performance of neutrophilic granulocytes and the relation to plasma insulin-like growth factor-I concentrations in dairy cows during the transition period. J. Dairy Sci. 94:1762-1771.

Sandgren, C.H., I. Larsson, and K. Persson. 1992. Bovine neutrophils recruited by endotoxin to a teat cistern continuously produce oxygen radicals and show increased phagocytosis and extracellular chemiluminescence. Inflammation 16:117-133.

SAS. 2001. User's Guide: Statistics. 8th ed. SAS Inst. Inc., Cary, NC.

Scaletti, R.W., D. S. Trammell, B. A. Smith, and R. J. Harmon.2003. Role of dietary copper in enhancing resistance to Escherichia coli mastitis. J. Dairy Sci. 86:12401249.

Scalia, D., N. Lacetera, U. Bernabucci, K. Demeyere, L. Duchateau, and C. Burvenich. 2006. In vitro effects of nonesterified fatty acids on bovine neutrophils oxidative burst and viability. J. Dairy Sci. 89:147-154.

Schenten, D., and R. Medzhitov. 2011. The control of adaptive immune responses by the innate immune system. Adv. Immunol. 109:87-124.

Schmidt, E. P., W. L. Lee, R. L. Zemans, C. Yamashita, and G. P. Downey. 2011. On, around, and through: neutrophil-endothelial interactions in innate immunity. Physiology 26:334-347.

Schramm, R. D., S. L. Shields, P. Rezamand, K. S. Seo, and M. A. McGuire. 2011. Effects of OmniGen-AF on blood neutrophil gene expression, milk leucocyte 
populations and production performance of early lactating dairy cows. J. Anim. Physiol. Anim. Nutr. doi: 10.1111/j.1439-0396.2011.01190.x.

Schwab, E. C., C. G. Schwab, R. D. Shaver, C. L. Girard, D. E. Putnam, and N. L. Whitehouse. 2006. Dietary forage and nonfiber carbohydrate contents influence B-vitamin intake, duodenal flow, and apparent ruminal synthesis in lactating dairy cows. J. Dairy Sci. 89:174-187.

Segal, A. W. 2005. How neutrophils kill microbes. Annu. Rev. Immunol. 23:197-223.

Shafer-Weaver, K. A., G. M. Pighetti, and L. M. Sordillo. 1996. Diminished mammary gland lymphocyte functions parallel shifts in trafficking patterns during the postpartum period. Proc. Soc. Exp. Biol. Med. 212:271-280.

Shankar, A. H, and A. S. Prasad. 1998. Zinc and immune function: the biological basis of altered resistance to infection. Am. J. Clin. Nutr. 68:447S-463S.

Sharma, M. C., C. Joshi, N. N. Pathak, and H. Kaur. 2005. Copper status and enzyme, hormone, vitamin and immune function in heifers. Res. Vet. Sci. 79:113-123.

Shaver, R. D., and M. A. Bal. 2000. Effect of dietary thiamin supplementation on milk production by dairy cows. J. Dairy Sci. 83:2335-2340.

Shepherd, V. L. 1986. The role of the respiratory burst of phagocytes in host defense. Semin. Respir. Infect. 1:99-106.

Sheppard, F. R., M. R. Kelher, E. E. Moore, N. J. McLaughlin, A. Banerjee, and C. C. Silliman. 2005. Structural organization of the neutrophil NADPH oxidase: phosphorylation and translocation during priming and activation. J. Leukoc. Biol. 78:1025-1042.

Shiloh, M. U., J. Ruan, and C. Nathan. 1997. Evaluation of bacterial survival and phagocyte function with a fluorescence-based microplate assay. Infect. Immun. 65:3193-198.

Shuster, D. E., E. K. Lee, and M. E. Kehrli, Jr. 1996. Bacterial growth, inflammatory cytokine production, and neutrophil recruitment during coliform mastitis in cows within ten days after calving, compared with cows at midlactation. Am. J. Vet. Res. 57:1569-1575.

Siciliano-Jones, J. L., M. T. Socha, D. J. Tomlinson, and J. M. DeFrain. 2008. Effect of trace mineral source on lactation performance, claw integrity, and fertility of dairy cattle. J. Dairy Sci. 91:1985-1995. 
Sitkovsky, M., and D. Lukashev. 2005. Regulation of immune cells by local-tissue oxygen tension: HIF1 alpha and adenosine receptors. Nat. Rev. Immunol. 5:712721.

Smith, K. L., D. A. Todhunter, and P. S. Schoenberger. 1985a. Environmental mastitis: cause, prevalence, prevention. J. Dairy Sci. 68:1531-1553.

Smith, K. L., D. A. Todhunter, and P. S. Schoenberger. 1985b. Environmental pathogens and intramammary infection during the dry period. J. Dairy Sci. 68:402-417.

Sohn, E. J., M. J. Paape, D. D. Bannerman, E. E. Connor, R. H. Fetterer, and R. R. Peters. 2007a. Shedding of sCD14 by bovine neutrophils following activation with bacterial lipopolysaccharide results in down-regulation of IL-8. Vet. Res. 38:95108.

Sohn, E. J., M. J. Paape, E. E. Connor, D. D. Bannerman, R. H. Fetterer, and R. R. Peters. 2007b. Bacterial lipopolysaccharide stimulates bovine neutrophil production of TNF- $\alpha$, IL-1 $\beta$, IL-12 and IFN- $\gamma$. Vet. Res. 38:809-818.

Sordillo, L. M., and S. L. Aitken. 2009. Impact of oxidative stress on the health and immune function of dairy cattle. Vet. Immunol. Immunopathol. 128:104-109.

Sordillo, L. M., G. A. Contreras, and S. L. Aitken. 2009. Metabolic factors affecting the inflammatory response of periparturient dairy cows. Anim. Health Res. Rev. 10:53-63.

Sordillo, L. M., N. O'Boyle, J. C. Gandy, C. M. Corl, and E. Hamilton. 2007. Shifts in thioredoxin reductase activity and oxidant status in mononuclear cells obtained from transition dairy cattle. J. Dairy Sci. 90:1186-1192.

Sordillo, L. M., G. M. Pighetti, and M. R. Davis. 1995. Enhanced production of bovine tumor necrosis factor-alpha during the periparturient period. Vet. Immunol. Immunopathol. 49:263-270.

Sordillo, L. M., M. J. Redmond, M. Campos, L. Warren, and L. A. Babiuk. 1991. Cytokine activity in bovine mammary gland secretions during the periparturient period. Can. J. Vet. Res. 55:298-301.

Sordillo, L. M., K. Shafer-Weaver, and D. DeRosa. 1997. Immunobiology of the mammary gland. J. Dairy Sci. 80:1851-1865.

Sordillo, L. M. and K. L. Streicher. 2002. Mammary gland immunity and mastitis susceptibility. J. Mammary Gland Biol. Neoplasia 7:135-146.

Spears, J. W. 2000. Micronutrients and immune function in cattle. Proc. Nutr. Soc. 59:587-594. 
Spears, J. W. 2003. Trace mineral bioavailability in ruminants. J. Nutr. 133:1506S1509 S.

Spears, J. W., and W. P. Weiss. 2008. Role of antioxidants and trace elements in health and immunity of transition dairy cows. Vet. J. 176:70-76.

Sprecher, D. J., D. E. Hostetler, and J. B. Kaneene. 1997. Locomotion scoring of dairy cattle. Theriogenology 47:1178-1187.

Stabel, J. R., J. P. Goff, and K. Kimura. 2003. Effects of supplemental energy on metabolic and immune measurements in periparturient dairy cows with Johne's disease. J. Dairy Sci. 86:3527-3535.

Ster, C., M.-C. Loiselle, and P. Lacasse. 2012. Effect of postcalving serum nonesterified fatty acids concentration on the functionality of bovine immune cells. J. Dairy Sci. 95:708-717.

Stevens, M. G., L. J. Peelman, B. De Spiegeleer, A. Pezeshki, G. R. Van De Walle, L. Duchateau, and C. Burvenich. 2011. Differential gene expression of the toll-like receptor-4 cascade and neutrophil function in early- and mid-lactating dairy cows. J. Dairy Sci. 94:1277-1288.

Straub, R. H. 2007. The complex role of estrogens in inflammation. Endocr. Rev. 28:521574.

Sultan, M., M. H. Schulz, H. Richard, A. Magen, A. Klingenhoff, M. Scherf, M. Seifert, T. Borodina, A. Soldatov, D. Parkhomchuk, D. Schmidt, S. O'Keeffe, S. Haas, M. Vingron, H. Lehrach, and M. L. Yaspo. 2008. A global view of gene activity and alternative splicing by deep sequencing of the human transcriptome. Science 321:956-960.

Sun, P., J. Wang, G. Yang, Khas-Erdene, and Q. Liu. 2010. Effects of different doses of free alpha-linolenic acid infused to the duodenum on the immune function of lactating dairy cows. Arch. Anim. Nutr. 64:504-513.

Suriyasathaporn, W., A. J. Daemen, E. N. Noordhuizen-Stassen, S. J. Dieleman, M. Nielen, and Y. H. Schukken. 1999. Beta-hydroxybutyrate levels in peripheral blood and ketone bodies supplemented in culture media affect the in vitro chemotaxis of bovine leukocytes. Vet. Immunol. Immunopathol. 68:177-186.

Suriyasathaporn, W., C. Heuer, E. N. Noordhuizen-Stassen, and Y. H. Schukken. 2000. Hyperketonemia and the impairment of udder defense: a review. Vet. Res. 31:397-412. 
Suttle, N. F., and D. G. Jones. 1989. Recent developments in trace element metabolism and function: trace elements, disease resistance and immune responsiveness in ruminants. J. Nutr. 119:1055-1061.

Tamura, J., K. Kubota, H. Murakami, M. Sawamura, T. Matsushima, T. Tamura, T. Saitoh, H. Kurabayshi, and T. Naruse. 1999. Immunomodulation by vitamin B12: augmentation of CD8+ T lymphocytes and natural killer (NK) cell activity in vitamin B12-deficient patients by methyl-B12 treatment. Clin. Exp. Immunol. 116:28-32.

Tanase, C. A. 2010. Histidine domain-protein tyrosine phosphatase interacts with Grb2 and GrpL. PLoS One 15:e14339.

Taylor, B. C., J. D. Dellinger, J. S. Cullor, and J. L. Stott. 1994. Bovine milk lymphocytes display the phenotype of memory $\mathrm{T}$ cells and are predominantly CD8+. Cell. Immunol. 156:245-253.

Tucker, H. A. 2000. Hormones, mammary growth, and lactation: a 41-year perspective. J. Dairy Sci. 83:874-884.

USDA. 2008. Dairy 2007 Part II: Changes in the US Dairy Cattle Industry, 1991-2007. USDA-Animal and Plant Health Inspection Service-Veterinary Services, Centers for Epidemiology and Animal Health, Fort Collins, CO.

Vande Walle, L., and M. Lamkanfi. 2011. Inflammasomes: caspase-1-activating platforms with critical roles in host defense. Front. Microbiol. 2:3-9.

Vangroenweghe, F., I. Lamote, and C. Burvenich. 2005. Physiology of the periparturient period and its relation to severity of clinical mastitis. Domest. Anim. Endocrinol. 29:283-293.

Velkeniers, B., Z. Dogusan, F. Naessens, R. Hooghe, and E. L. Hooghe-Peters. 1998. Prolactin, growth hormone and the immune system in humans. Cell. Mol. Life Sci. 54:1102-1108.

Waldron, M. R., A. E. Kulick, A. W. Bell, and T. R. Overton. 2006. Acute experimental mastitis is not causal toward the development of energy-related metabolic disorders in early postpartum dairy cows. J. Dairy Sci. 89:596-610.

Waldron, M. R., T. Nishida, B. J. Nonnecke, and T. R. Overton. Effect of lipopolysaccharide on indices of peripheral and hepatic metabolism in lactating cows. J. Dairy Sci. 86:3447-3459.

Wang, Y. Q., S. B. Puntenney, J. L. Burton, and N. E. Forsberg. 2007. Ability of a commercial feed additive to modulate expression of innate immunity in sheep immunosuppressed with dexamethasone. Animal 1: 945-951. 
Wang, Z., M. Gerstein, and M. Snyder. 2009a. RNA-Seq: a revolutionary tool for transcriptomics. Nat. Rev. Genet. 10:57-63.

Wang, Y. Q., S. B. Puntenney, J. L. Burton, and N. E. Forsberg. 2009b. Use of gene profiling to evaluate the effects of a feed additive on immune function in periparturient dairy cattle. J. Anim. Physiol. Anim. Nutr. 93:66-75.

Wartha, F., K. Beiter, S. Normark, and B. Henriques-Normark. 2007. Neutrophil extracellular traps: casting the NET over pathogenesis. Curr. Opin. Microbiol. 10:52-56.

Weber, P. S., S. A. Madsen, G. W. Smith, J. J. Ireland, and J. L. Burton. 2001. Pretranslational regulation of neutrophil L-selectin in glucocorticoid-challenged cattle. Vet. Immunol. Immunopathol. 83:213-240.

Wenz, J. R., G. M. Barrington, F. B. Garry, R. P. Ellis, and R. J. Magnuson. 2006. Escherichia coli isolates, serotypes, genotypes, and virulence genes and clinical coliform mastitis severity. J. Dairy Sci. 89:3408-3412.

Wedekind K. J., A. E. Hortin, and D. H. Baker. 1992. Methodology for assessing zinc bioavailability: efficacy estimates for zinc-methionine, zinc sulfate, and zinc oxide. J. Anim. Sci. 70:178-187.

Werling, D., J. Piercy, and T. J. Coffey. 2006. Expression of TOLL-like receptors (TLR) by bovine antigen-presenting cells--Potential role in pathogen discrimination? Vet. Immunol. Immunopathol. 112:2-11.

Wilson, D. J., and R. N. González. 2003. Vaccination strategies for reducing clinical severity of coliform mastitis. Vet. Clin. North Am. Food Anim. Pract. 19:187197.

Wilson, D. J., R. N. Gonzalez, J. Hertl, H. F. Schulte, G. J. Bennett, Y. H. Schukken, and Y. T. Grohn. 2004. Effect of clinical mastitis on the lactation curve: a mixed model estimation using daily milk weights. J. Dairy Sci. 87:2073-2084.

Wintergerst, E. S., S. Maggini, and D. H. Hornig. 2007. Contribution of selected vitamins and trace elements to immune function. Ann. Nutr. Metab. 51:301-323.

Winters, K. R., E. Meyer, V. M. Van Merris, W. L. Van Den Broeck, L. Duchateau, and C. Burvenich. 2003. Sex steroid hormones do not influence the oxidative burst activity of polymorphonuclear leukocytes from ovariectomized cows in vitro. Steroids 68:397-406.

Woodfin, A., M. B. Voisin, and S. Nourshargh. 2010. Recent developments and complexities in neutrophil transmigration. Curr. Opin. Hematol. 17:9-17. 
Worku, M. and Morris, A. 2009. Binding of different forms of lipopolysaccharide and gene expression in bovine blood neutrophils. J. Dairy Sci. 92:3185-3193.

Xin, Z., D. F. Waterman, R. W. Hemken, and R. J. Harmon. 1991. Effects of copper status on neutrophil function, superoxide dismutase, and copper distribution in steers. J. Dairy Sci. 74:3078-3085.

Yu, C., Z. R. Shi, C. Y. Chu, K. H. Lee, X. Zhao, and J. W. Lee. 2010. Expression of bovine granulocyte chemotactic protein-2 (GCP-2) in neutrophils and a mammary epithelial cell line (MAC-T) in response to various bacterial cell wall components. Vet. J. 186:89-95.

Zachut, M., A. Arieli, H. Lehrer, L. Livshitz, S. Yakoby, and U. Moallem. 2010. Effects of increased supplementation of n-3 fatty acids to transition dairy cows on performance and fatty acid profile in plasma, adipose tissue, and milk fat. J. Dairy Sci. 93:5877-5889.

Zhang, E. Y., B. L. Parker, and T. M. Yankee. 2011. Gads regulates the expansion phase of CD8+ T cell-mediated immunity. J. Immunol. 186:4579-4589.

Zhao, X. and P. Lacasse. 2008. Mammary Tissue Damage during Bovine Mastitis: Causes and Control. J. Anim. Sci. 86: 57-65.

Ziv, G., I. Hartman, E. Bogin, J. Abidar and A. Saran. 1976. Endotoxin in blood and milk and enzymes in the milk of cows during experimental Eschericia coli endotoxin mastitis. Theriogenology 6:343-352. 


\section{VITA}

Xavier S. Revelo was born in Quito, Ecuador on January 27, 1984. While attending high school in the city, he spent his weekends and summers at his family farm where he was first exposed to agriculture and dairy cows. Xavier then relocated to Honduras in 2002 where he obtained his Bachelor of Science degree in Agricultural Sciences from Zamorano University in December, 2005. Upon completion of his Bachelor's degree, he decided to pursue a graduate degree in Animal Sciences. Xavier attended the University of Vermont between January 2006 and July 2008. He was a graduate student under the mentorship of Dr. Thomas B. McFadden during his first semester at UVM and then under the supervision of Dr. Matthew R. Waldron until completion of his program. He received his Master of Science degree from UVM in July 2008. He then pursued a doctoral degree in the Division of Animal Sciences at The University of Missouri under the guidance of Dr. Matthew R. Waldron. Throughout his graduate program, Xavier focused his research on the biology of the neutrophil in the periparturient dairy cow. He actively participated in numerous scientific meetings and is author of several scientific abstracts and peer-reviewed publications. Xavier received the Doctor of Philosophy degree from the University of Missouri in June 2012. He is currently a post-doctoral fellow at the Toronto General Research Institute - University of Toronto where he investigates the role of immune cells in the development of human metabolic disease during obesity. 